\title{
Abstracts of the 2014 Annual Meeting of the \\ Lumbar Spine Research Society \\ Chicago, Illinois • May 1-2, 2014 \\ (DOI: 10.3171/2014.4.FOC-LSRSabstracts)
}

\section{Podium Presentation Abstracts}

\author{
Paper 1. Long-Term Outcomes after Non-Instrumented Lumbar \\ Arthrodesis
}

\begin{abstract}
Mohamad Bydon, M.D.; David Santiago-Dieppa, M.D.; Risheng Xu, A.M., Ph.D.; Rafael De la Garza-Ramos, M.D.; Mohamed Macki, B.S.; Robby Weingart; Daniel M. Sciubba, M.D.; Jean-Paul Wolinsky; Ali Bydon, M.D.; Ziya L. Gokaslan, M.D.; Timothy F. Witham
\end{abstract}

\section{Johns Hopkins University School of Medicine, Neurosurgery}

Disclosures: M. Bydon: None. D. Santiago-Dieppa: None. R. Xu: None. R. De la Garza-Ramos: None. M. Macki: None. R. Weingart: None. D.M. Sciubba: 4; I; Medtronic, Nuvasiv, Globus, DePuy. 7; I; DePuy. J. Wolinsky: None. A. Bydon: 4; I; MedImmune, LLC. 7; I; DePuy. Z.L. Gokaslan: 6; I; Spinal Kinetics, US Spine. 7; I; DePuy, AOSpine North America, Medtronic, NREF, Integra Life Sciences, K2M. T.F. Witham: 7; I; Eli Lilly and Company.

Introduction: The objective of this study is to examine the longterm outcomes of patients undergoing non-instrumented posterolateral fusion of the lumbar spine.

Methods: We present 376 patients who underwent in situ, noninstrumented arthrodesis for lumbar degenerative disease over a 20 -year period at a single institution. Patients were followed for an average of $83.2 \pm 65.5$ months after the index lumbar arthrodesis procedure.

Results: The average age for these patients was $61.1 \pm 13.54$ years, and $185(49.2 \%)$ patients were male. Of the total 376 patients, 344 $(91.5 \%)$ presented with back pain, $304(95.9 \%)$ with radiculopathy, $20(5.32 \%)$ with motor weakness, and $7(1.86 \%)$ with pre-operative bowel/bladder dysfunction. The most common pre-operative diagnosis was multi-level lumbar stenosis with claudication in 211 (56.1\%) patients, followed by degenerative disc disease in 111 (29.5\%) patients. An average of $1.76 \pm 0.82$ spinal levels were fused. Autograft was used in $345(91.75 \%)$ patients, while allograft was utilized in $31(8.24 \%)$ patients. Average blood loss was $625 \pm 487$ milliliters. 15 (3.99\%) patients experienced intra-operative durotomy during the surgery. Peri-operatively, the average length of stay was $5.98 \pm 5.78$ days. Post-operatively, patients experienced a significant improvement in back pain $(\mathrm{p}<0.0001)$ and radiculopathy $(\mathrm{p}<0.0001)$. At last follow-up, $228(60.64 \%)$ patients experienced continued or recurrent back pain, while $217(57.71 \%)$ patients experienced continued or recurrent radiculopathy. The cumulative rate of adjacent segment disease development over time was $18.35 \%$ (69 patients). The pseudoarthrosis rate was $5.59 \%$ (21 patients). In total, the rate of reoperation due to non-improvement or worsening of symptoms was $30.59 \%$ (115 patients).

Discussion: The utility and outcomes of non-instrumented lumbar fusions is controversial in the literature. In this manuscript, we present one of the largest cohorts of patients undergoing in situ fusion for degenerative lumbar spine disease. We also present a cohort with one of the longest follow-up times, an average of 6.9 years.

Conclusions: Patients undergoing non-instrumented fusion have statistically significant improved back pain and radiculopathy postoperatively. Notably, while $18.35 \%$ of patient developed adjacent segment disease, $30.6 \%$ of patients required re-operation due to recurrent or worsening symptoms during the follow-up period.
Paper 2. Lumbar Spine Fusion Rates with Local Bone in Posterolateral and Combined Posterolateral and Interbody Approaches: Results from a Multicenter Trial

Daniel Park, MD; Kevin Baker; Paul Arnold, MD, FACS; David Kim, MD; Rick Sasso, MD; Jeff Fischgrund, MD

Beaumont Health System, Orthopaedic Research

Disclosures: D. Park: 4; I; Stryker, Depuy. 6; I; Depuy. K. Baker: 7; I; Globus Medical. P. Arnold: 4; I; Cerapedics, Medtronic, Life Spine, Integra Life, SpineWave, Stryker Spine, AO Spine. 7; I; Cerapedics, Depuy Spine, AO Spine North America, Covidien, Institute for Advancing Medical Innovation, Asubio, Spineology, Relievant, LANX, BHR Pharma. D. Kim: 4; I; Bioventus, Pioneer. R. Sasso: 1; I; Medtronic. 4; I; Globus Medical. 6; I; Biomet, SpineCor, Trans1. 7; I; Cerapedics, Medtronic, Smith \& Nephew, Stryker. 9; I; Saunders-Mosby/Elsevier. J. Fischgrund: 3; I; Baxano Surgical (Board of Directors). 4; I; Stryker, Relievant, Baxter, Fziomed. 6; I; Baxano Surgical. 8; I; Stryker. 9; I; JAAOS.

Introduction: Posterolateral lumbar fusion is the most common method used to treat degenerative lumbar conditions. Local bone harvested from the laminectomy has been used as an alternative to iliac crest because it is easily accessed and is not associated with complications. Despite ease of access, fusion outcomes using only local bone is wanting.

Materials \& Methods: A retrospective review of 241 patients treated with instrumented posterolateral lumbar fusion (PLF), or PLF with interbody fusion (PLF+IF) at a single level in association with a multi-center trial was undertaken. Local autologous bone was used as the fusion material, without any additional extenders/enhancers. Fusion was assessed by two radiologists at 6 and 12 months using three criterion: 1 .) $>3 \mathrm{~mm}$ of translation and 2.) $>5$ o of angulation on flexion-extension radiographs and 3). qualitative grading of bridging bone via computed tomography $(\mathrm{CT})$ scans.

Results: 25 patients out of 145 (18.2\%) treated with PLF alone were graded as fused bilaterally at 6 months, while 33 patients (24.1\%) demonstrated at least unilateral fusion. One patient out of 96 $(1.1 \%)$ treated with PLF+IF was graded as successfully fused at all three fusion sites at 6 months, while 10 patients $(10.6 \%)$ treated with PLF+IF demonstrated a solid interbody fusion, and 8 patients $(8.5 \%)$ had at least one posterolateral side of the spine fused. 48 patients out of $115(44.4 \%)$ treated with PLF, had bilateral fusion at 12 months. 57 patients $(52.7 \%)$ had at least unilateral fusion. 18 patients out of $76(24.3 \%)$ treated with PLF+IF demonstrated successful interbody fusion, and 19 patients $(25.7 \%)$ had at least one posterolateral side of the spine fused.

Discussion: Lumbar fusion using local bone alone without bone graft enhancer and extenders provide a surprisingly low fusion rate. Because the volume of local bone is limited, the fusion rate is approximately half the rate $(25.7 \%$ versus $52.7 \%)$ when augmented with interbody. Our study was a multicenter study likely representing the community rather than a single surgeon experience.

Conclusions: The use of local bone alone provides a fusion rate substantial less than fusions using the "goal standard" iliac crest autograft with or without an interbody cage. 


\section{Abstracts of the 2014 Meeting of the Lumbar Spine Research Society}

Paper 3. The Role Of Dioxin In Smoking-mediated Bone Healing Inhibition

Sharath S. Bellary, M Eng, MD; Kevin A. Sonn, BS; Sohaib Z. Hashmi, BS; John T. Nelson, MD; Amruta Ashtekar, MS; Christian J. Park, BS; Chawon Yun, PhD; Michael S. Nikoli, MD; Jason H. Ghodasra, MD; Wellington K. Hsu, MD; Erin L. Hsu, PhD

\section{Northwestern University- Dept of Orthopedics}

Disclosures: S.S. Bellary: None. K.A. Sonn: None. S.Z. Hashmi: None. J.T. Nelson: None. A. Ashtekar: None. C.J. Park: None. C. Yun: None. M.S. Nikoli: None. J.H. Ghodasra: None. W.K. Hsu: 4; I; Stryker, Zimmer, Medtronic, Pioneer Surgical, Terumo, Spinesmith. 7; I; Medtronic, Pioneer Surgical, Baxter. E.L. Hsu: 4; I; Stryker, Zimmer, Medtronic, Pioneer Surgical, Terumo, Spinesmith. 7; I; Medtronic, Pioneer Surgical, Baxter.

Introduction: Smoking inhibits bone healing, leading to increased rates of pseudoarthrosis. The mechanism behind these effects is controversial. Dioxin negatively impacts bone quality and is suggested to affect osteoblast differentiation. We hypothesized that dioxin inhibits osteogenesis via the Aryl Hydrocarbon Receptor (AHR), and that dioxin treatment would inhibit arthrodesis in a rat posterolateral fusion (PLF) model.

Methods: A wound assay, whereby rat bone marrow stromal cells (BMSC) migrate across a scratched monolayer, was performed to evaluate the rate of cell migration after treatment with DMSO, dioxin, nicotine, alpha-Naphthoflavone (ANF, an AHR antagonist), or dioxin+ANF. An alkaline phosphatase (ALP) assay was also performed to quantify enzyme activity. For in vivo studies, female Long-Evans rats were injected weekly with dioxin or vehicle control for 6 weeks prior to PLF across L4 and L5 using $1 \mu \mathrm{g}$ rhBMP-2 on an absorbable collagen sponge (ACS). Treatments continued until sacrifice at 4 weeks post-op. Spines were evaluated using radiographs, microCT, and fusion scoring.

Results: Dioxin inhibited cell migration in vitro, and ANF co-treatment rescued the effect; nicotine did not reduce migration rate. Dioxin decreased ALP activity, and ANF similarly rescued this inhibitory effect. Radiographs showed decreased bridging bone formation in dioxin-treated rats. Fusion scores and rates were significantly lower in dioxin-treated rats relative to controls ( 1.73 vs $0.71, \mathrm{p}<0.001$; $100 \%$ vs $50 \%$, p $<0.01$; Figure $1 \mathrm{~A}-\mathrm{B})$. MicroCT quantification and 3 -D reconstruction showed no change in new bone formation in fused spines between groups (Figure 1C).

Discussion: Our in vitro studies suggest that dioxin inhibits preosteoblast cell migration and ALP activity through activation of the
AHR. As such, the AHR may represent a therapeutic target for the protection of smokers against bone healing inhibition. Dioxin significantly lowered fusion scores and rates in a rat arthrodesis model. Future studies will determine whether AHR antagonists protect against dioxin action in vivo.

Conclusions: Although smoking causes complications in bone healing, the mechanisms remain elusive. Our study suggests that dioxin-like compounds may be responsible for these inhibitory effects, and that agents which block the AHR may have therapeutic potential in the smoking population.

Paper 4. Laminectomy with Fusion is cost-effective versus laminectomy alone for Grade I Degenerative Lumbar Spondylolisthesis with Spinal Stenosis: Cost-Utility Analysis over a 5 year time horizon

Robert Whitmore, MD; Ryan Din, BS; Edward C. Benzel, MD; Frederick G. Barker, MD; Subu N. Magge, MD; Jean V. Coumans, MD; J. Sanford Schwartz, MD; Zoher Ghogawala, MD

\section{Lahey Hospital \& Medical Center, Neurosurgery}

Disclosures: R. Whitmore: None. R. Din: None. E.C. Benzel: 4; I; AxioMed, OrthoMEMS. 6; I; AxioMed, OrthoMEMS, Turning Point. 9; I; Elsevier Publishing, Thieme Publishing. F.G. Barker: None. S.N. Magge: None. J.V. Coumans: None. J.S. Schwartz: 4; I; Bayer, Genentech. Z. Ghogawala: None.

Objective: Grade I degenerative lumbar spondylolisthesis with spinal stenosis is a common cause of spinal morbidity. It remains unclear whether to treat this condition with laminectomy alone or lumbar fusion with laminectomy. This study reports a cost-utility analysis of these two procedures at five-year follow-up.

Methods: Patients were obtained from a single site participating in a randomized trial comparing patients aged $50-81$ years, receiving laminectomy alone or laminectomy with instrumented fusion between 2002-2009. Short Form-36 (SF-36) and Oswestry Lower Back Disability Index (ODI) were collected prior to surgery and post-operatively at 3 months, 6 months, 1 year, and annually to 5 years. Direct costs were calculated from a hospital perspective using total charges and year-specific cost-to-charge ratios, adjusted to 2012 dollars. Short Form-6D (SF-6D) utility scores were computed from the SF-36, and gains in Quality-Adjusted Life-Years (QALYs) were calculated by taking the area under the utility curve relative to the baseline.

Results: 39 patients received laminectomy and 37 patients received
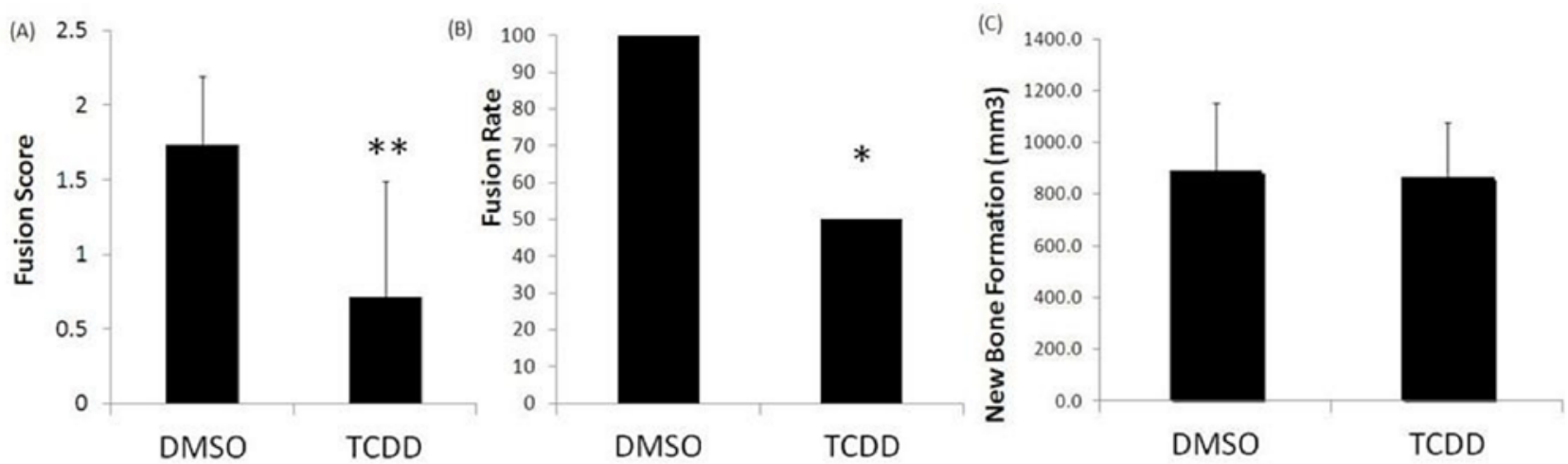

Figure 1. Fusion scores (A) and rates (B) for control- and dioxin-treated

$$
\text { rats. }{ }^{*} p<0.01,{ }^{* *} p<0.01
$$

(C) microCT quantification of new bone formation across L4-L5 of fused 


\section{Abstracts of the 2014 Meeting of the Lumbar Spine Research Society}

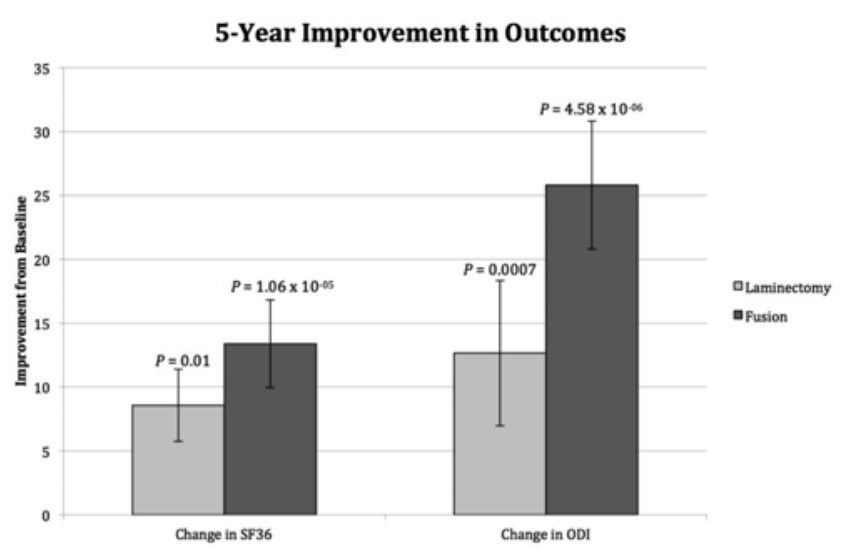

laminectomy with fusion. All patients showed significant improvements in the SF-36 and ODI relative to baseline $(\mathrm{p}<0.01)$. Patients receiving fusion showed greater gain in QALYs at 5 years compared to laminectomy alone $(0.83+0.20$ vs. $0.46+0.24 ; p=0.001)$. Patients who received laminectomy alone had higher reoperation rate $(38.5 \%$ vs. $18.9 \% ; \mathrm{p}=0.06$ ). Total costs, including reoperations, were lower for laminectomy alone compared to fusion $(\$ 23,428+\$ 18,651 \mathrm{vs}$. $\$ 41,019+\$ 17,164, \mathrm{p}<0.00006)$. Cost/QALY ratios were comparable for each procedure (laminectomy: $\$ 50,297$; fusion: $\$ 49,498$ ). Incremental cost-effectiveness ratio (ICER) of fusion compared to laminectomy alone was $\$ 48,473$, and below societal willingness-topay threshold.

Conclusions: Laminectomy with fusion is associated with greater gain in QALYs at 5 years compared to laminectomy alone and is a cost-effective procedure for Grade I degenerative lumbar spondylolisthesis.

\section{Paper 5. The Fate of L5-S1 with Low Dose BMP-2 and Pelvic Fixation in Adult Deformity Surgery}

Prokopis Annis, MD; William R. Spiker, MD; Brandon D.Lawarence, MD; Michael D. Daubs, MD; Darrel S. Brodke

\section{University of Utah, Department of Orthopaedics}

Disclosures: P. Annis: None. W.R. Spiker: None. B.D. Lawarence: None. M.D. Daubs: 1; I; Synthes Spine. 4; I; Synthes Spine. D.S. Brodke: 1; I; Amedica, DePuy Synthes, Medtronic. 4; I; DePuy Synthes. 6; I; Amedica, Vertiflex.

Introduction: Pseudarthrosis at the L5-S1 level is one of the most common complications of long fusions to the sacrum in adult deformity patients. Strategies for decreasing non-union rates include interbody fusion and cage, use of high-dose BMP at the Lumbo-sacral junction, and the use of sacro-pelvic fixation. A recent study found comparable fusion rates with interbody fusion or high-dose BMP posterolaterally. Further questions arise as to whether sacro-pelvic fixation and lower-dose BMP can also supplant the use of interbody fusion. The purpose of the study was to evaluate L5-S1 fusion success after adult deformity surgery with pelvic fixation and low dose BMP2 , with or without interbody fusion.

Methods: Retrospective review of 61 consecutive patients with minimum 2-year follow up, treated operatively at a single institution for adult spinal deformity. Inclusion criteria were posterior approach only, $\geq 5$ levels fused, non-previous spine procedures at L5-S1, and use of pelvic fixation. The patients were divided in 2 groups for comparison based on the use of interbody cage at L5-S1 level. Radiographic union was assessed by 3 independent reviewers using Bridwell s criteria. Revision rates and implant related complications were also reported.
Results: The mean age of the 61 patient cohort was 63 years (25-80) and mean follow-up was 38 months (24-90). There were 35 patients in the interbody group, 26 in the no interbody group. The mean amount of BMP-2 inserted in the disc space was $2.5 \mathrm{mg}(0-8)$. In both groups a mean of $3.5 \mathrm{mg}(2-4)$ was used posterolaterally with allograft and local autograft. There were no significant differences in the radiographic parameters or deformity correction between interbody and non-interbody groups. Fusion rate at L5-S1 was found to be $95 \%(58 / 61)$ with no difference between the 2 groups (94.3\% vs $96.2 \%, \mathrm{p}=0.6$ ) and the overall revision rate for L5-S1 nonunion $1.6 \%$. There were 5 cases of prominent/painful iliac screw removal.

Conclusions: The use of low dose of BMP-2 at the L5-S1 level in combination with sacro-pelvic fixation achieved satisfactory outcomes in adult deformity surgery. No additional benefit was encountered by adding additional interbody cage and fusion procedures.

Paper 6. Comparative Outcomes and Cost-Utility following Surgical Treatment of Focal Lumbar Spinal Stenosis Compared with Osteoarthritis of the Hip or Knee: Long-Term Change in Health-Related Quality of Life.

Y. Raja Rampersaud; Stephen Lewis; Rajiv Gandhi, MS, MD FRCSC; Rod Davey; Nizar Mohamed

University Health Network, Toronto Western Hospital, University of Toronto, Orthopaedics

Disclosures: Y. Rampersaud: 5; I; Medtronic. S. Lewis: 2; I; Stryker, Medtronic. 4; I; Medtronic. R. Gandhi: 5; I; Eli Lilly. 7; I; Smith and Nephew, Biomet. R. Davey: 1; I; Biomet. 2; I; Biomet. 4; I; Biomet. N. Mohamed: 2; I; Smith and Nephew.

Introduction: To assess whether improvements in health related quality of life (HRQoL) following surgical management of focal lumbar spinal stenosis (FLSS) with or without spondylolisthesis is sustainable over the long-term compared to that of hip and knee arthroplasty (THA/TKA) for osteoarthritis (OA).

Methods: A single-center, retrospective longitudinal matched cohort study of prospectively collected outcomes at a minimum follow-up of 5 years . Cohorts: primary 1-2 level spinal decompression with or without instrumented fusion for FLSS(n=99), THA(n=99) and TKA(n=99). Postoperative change from baseline to last follow-up in SF-36 physical (PCS) and mental (MCS) component summary scores between groups was the primary outcome measure.

Results: Mean follow-up in months (\%follow-up) FLSS/THA/ TKA was $80.5+/-16.04(79 \%) / 94.6+/-16.62(92 \%) / 80.6+/-16.84$ $(85 \%)$ respectively (range of 5-10 years). Number of patients having undergone revision, including those lost to follow-up, FLSS/THA/ TKA was $n=20(20.2 \%$ - same site $(n=7)$ and adjacent segment $(\mathrm{n}=13))$ requiring 27 operations $/ \mathrm{n}=3(3 \%)$ requiring 5 operations/ $\mathrm{n}=8(8.1 \%)$ requiring 12 operations $(\mathrm{p}<0.01)$. The average time to first revision was 56/65/43 months, respectively. Mean postoperative PCS $(p<0.0001)$ and MCS $(p<0.02)$ improved significantly and was durable for all groups at the last follow-up. The mean change from baseline PCS/MCS to last follow-up, was 8.5/6.4, 12.3/7.0 and 8.3/4.9 for FLSS, THA and TKA respectively. Adjusting for baseline age, sex, BMI, PCS and MCS, there was a strong trend in favour of greater sustained change in the PCS of THA over FLSS $(p=0.07)$ and TKA $(\mathrm{p}=0.08)$. There was no difference in the degree of change in PCS between FLSS and TKA $(\mathrm{p}=0.95)$ and MCS between all three cohorts $(\mathrm{p}>0.1)$.

Conclusions: Despite a higher revision rate, patients undergoing surgery for FLSS can expect a comparable long-term (7-8 years) average improvement in $\mathrm{HRQoL}$ from baseline compared to their peers undergoing TKA and to a lesser extent THA. 


\section{Abstracts of the 2014 Meeting of the Lumbar Spine Research Society}

\section{Paper 7. Operative Treatment of Combat-Related Spine Trauma (CReST) During the Conflicts in Iraq and Afghanistan}

Daniel G. Kang; Scott C. Wagner, M.D.; Gregory S. Van Blarcum, M.D.; Ronald A. Lehman, M.D.

Walter Reed National Military Medical Center, Orthopaedic Surgery

Disclosures: D.G. Kang: None. S.C. Wagner: None. G.S. Van Blarcum: None. R.A. Lehman: 7; I; Depuy Synthes Spine, Centinel Spine.

Introduction: Several recent studies have examined the rates of combat-related spinal injury sustained in Operations Enduring and Iraqi Freedom using joint trauma registries. Despite these epidemiologic studies, a shortcoming of registry data collection has been limited clinical information after surgical intervention. Therefore, we set out to describe the operative treatment of combat-related spine trauma over a ten-year period at three high-volume military treatment facilities.

Methods: A retrospective analysis of a surgical database at three military institutions was performed; patients undergoing spine surgery designated in as engaged in Operations Enduring and/or Iraqi Freedom between 01JUL2003 and 01JUL2013 were evaluated. Inclusion criteria included trauma sustained in direct relation to combat operations while in theater requiring operative treatment after evacuation to Walter Reed Army Medical Center, National Naval Medical Center, or the combined Walter Reed National Military Medical Center (after August 2011). Demographic information, mechanism of injury and nature/location of spine injuries were recorded for all service members identified as undergoing surgery for combat-related spine trauma.

Results: 302 patients with combat-related (OIF/OEF) spine trauma requiring operative intervention were identified. 105 casualties required definitive surgical management after return to the United States. The mean age of these casualties was 29.8 years. $74.3 \%$ of these casualties were enlisted US Army servicemembers. $49.5 \%$ and $48.6 \%$ of injuries occurred in Afghanistan and Iraq, respectively. The most common mechanism of injury was mounted improvised explosive device (IED, 42.9\%). The lumbar spine was the most commonly involved region $(59 \%)$, followed by thoracic $(43.8 \%)$, cervical $(33.3 \%)$ and sacral (17.1\%). 1.5 spinal regions were injured per patient, and two patients sustained injuries to all four spinal regions. Spinal cord injuries were present in $29.5 \%$ of all patients. The mortality rate for all patients after evacuation to the United States was $1.9 \%$.

Conclusions: The incidence of combat-related spine trauma during the current conflicts in Iraq and Afghanistan is the highest in recorded history. This current retrospective study attempts to evaluate the demographic information, resource utilization and longer-term follow up data for patients sustaining war-related spine trauma requiring operative treatment.

Paper 8. Comparative Outcomes and Cost-Utility following Surgical Treatment of Focal Lumbar Spinal Stenosis Compared with Osteoarthritis of the Hip or Knee: Part 2- Estimated Lifetime Incremental Cost-Utility Ratios.

Y. Raja Rampersaud; Peggy Tso; Kevin Walker; Stephen Lewis; Rajiv Gandhi; Rod Davey; Nizar Mohamed; Peter Coyte

University Health Network, Toronto Western Hospital, University of Toronto, Orthopaedics

Disclosures: Y. Rampersaud: 5; I; Medtronic. P. Tso: None. K. Walker: None. S. Lewis: 2; I; Stryker, Medtronic. 5; I; Medtronic. R. Gandhi: 4; I; Eli Lilly. 7; I; Smith and Nephew, Biomet. R. Davey: 1; I; Biomet. 2; I; Biomet. 4; I; Biomet. N. Mohamed: 2; I; Smith and Nephew. P. Coyte: None.
Introduction: To estimate the lifetime incremental cost-utility ratios (ICUR) for decompression (D) and decompression with fusion (DF) for focal lumbar spinal stenosis (FLSS) versus total hip and knee arthroplasty (THA/TKA) for osteoarthritis (OA) from hospital perspective based on long-term health status data at a median of 5 years (4-7 years) post-surgical intervention.

Methods: A single-center, retrospective longitudinal matched cohort study of prospectively collected outcomes and retrospectively collected costs including patients with primary 1-2 level D or DF for FLSS $(n=99)$ and THA(n=99)/TKA(n=99) for primary OA. ICUR (\$/QALY) determined using perioperative costs (direct and indirect) and Short Form-6D (SF-6D) utility scores converted from the SF-36.

Utility modeled over the lifetime and quality-adjusted-life-years (QALY) determined using the median 5-year health status data. Primary outcome measure, cost per QALY gained, calculated by estimating the mean incremental lifetime costs and QALYs for each diagnosis group after discounting costs and QALYs at 3\%. Sensitivity analyses adjusting for, $+25 \%$ primary and revision surgery cost, + $25 \%$ revision rate, upper and lower confidence interval utility score, variable inpatient rehabilition rate for THA/TKA and discounting at $5 \%$, were conducted to determine factors affecting the value of each type of surgery.

Results: Follow-up and revision surgery data attained for $85 \%$ FLSS, 80\%-THA and 75\%-THA of the cohorts. 5-year ICURs were \$21,702/QALY for THA; \$28,595/QALY for TKA; \$12,271/QALY for D; and $\$ 35,897 / \mathrm{QALY}$ for DF. Estimated lifetime ICURs using the median 5-year follow-up data was $\$ 5,682 / \mathrm{QALY}$ for THA; $\$ 6,489 / \mathrm{QALY}$ for TKA; $\$ 2,994 / \mathrm{QALY}$ for D; and \$10,806/QALY for DF. The overall spine (D and DF) ICUR was $\$ 5,617 / \mathrm{QALY}$. The estimated best and worst-case lifetime ICURs varied from $\$ 1,126$ / QALY for the best-case (D) to $\$ 39,323 / \mathrm{QALY}$ for the worst case (DF).

Conclusions: Surgical management of primary OA of the spine, hip and knee results in durable cost-utility ratios that are well below accepted thresholds for cost-effectiveness.

Paper 9. Postoperative Leg Pain And/or Dysesthesisa At One Year Follow-up Following Extreme Lateral Interbody Fusion At A Single Site

Jim A. Youssef; Douglas Orndorff, MD; Morgan Scott, MS; Rachel Ebner, MS; Sam Antoine; Allison Knewitz

\section{Durango Orthopedic Assoc/Spine Colorado}

Disclosures: J.A. Youssef: 1; I; Nuvasive, Integra, Osprey Biomedical, Amedica. 4; I; Nuvasive, Integra, Amedica. 6; I; Amedica, Vertiflex, Benvenue, Paradigm Spine, Promethean Surgical, ISD, spinicity, Spinal Ventures. 7; I; Vertiflex, Globus Medical, Nuvasive, Integra. D. Orndorff: 1; I; Integra. 4; I; Nuvasive, Integra, Stryker, Amedica. 7; I; Nuvaive, Integra, Vertiflex, Globus Medical. M. Scott: None. R. Ebner: None. S. Antoine: None. A. Knewitz: None.

Introduction: The use of XLIF (extreme lateral interbody fusion) as an effective minimally invasive treatment for spinal disorders is increasing in popularity. However, postoperative leg pain and/or dysesthesia are common complaints following this procedure. We report the outcomes following an XLIF at 1-year follow up: VAS, ODI, and postoperative leg pain and/or dysesthesia.

Methods: Local IRB approved, prospective, non-randomized single center study review of 195 patients with lumbar degenerative disorders, mean age of 66.3 years (30-87), from 2007 to 2013. The mean follow-up was 16.9 months. (1.32-78.3 mos). Patient-reported postoperative leg pain and/or dysesthesia for 195 patients (numbness, tingling, quadriceps weakness, leg spasms, anterior thigh, lateral leg, groin and hip pain) was recorded at 2-weeks, 6-weeks, 3-months, 


\section{Abstracts of the 2014 Meeting of the Lumbar Spine Research Society}

6-months, and 1-year postoperatively. VAS and ODI scores were collected preoperatively and at the 1-year follow up for the patients. 82 patients underwent a 1-level XLIF, 63 at 2 levels and 50 at 3 levels. 38 patients received anterior instrumentation: an interbody implant without instrumentation (24); a tabbed interbody implant with vertebral body screws (10); and an interbody implant and adjuvant titanium plate with vertebral body screws (4). 157 patients received supplemental posterior spinal fusion (PSF) with decompression.

Results: At the 2-week follow-up, 108 (56.6\%) of patients reported postoperative leg pain following the XLIF procedure. At 3 months, 52 $(27.2 \%)$ continued to report postoperative leg pain. At 6 months, 36 (20.8\%) still reported pain. At 1 year, only 19 (13.6\%) continued to report lower extremity pain and discomfort. Of the patients that provided VAS and ODI scores, scores were improved from baseline by an average of 38.3 ( 58 to 19.7 ) and 16.3 (38.9 to 22.6) points respectively; a clinically significant improvement. Rates of improvement were similar among patients who received anterior alone and anterior with posterior fixation.

Discussion: Postoperative leg pain and/or dysesthesia are a common patient complaint following XLIF surgery. Our study reports resolution of $86.4 \%$ of postoperative leg pain at 1-year follow-up.

Conclusions: Postoperative leg symptoms appear to be approach related and self-limiting following an XLIF procedure. Further understanding of this approach related issue warrants investigation.

Paper 10. Intraoperative Vancomycin Use in Spinal Surgery: Single Institution Experience and Microbial Trends

George M. Ghobrial, MD; Vismay Thakkar, MD; Edward Andrews; Michael J. Lang, MD; Ameet Chitale, MD; Mark E. Oppenlander, MD; Christopher Maulucci, MD; Ashwini D. Sharan, MD; James S. Harrop, MD; Jack Jallo, MD, PhD; Srinivas Prasad, MD

\section{Thomas Jefferson University Hospital, Neurological Surgery}

Disclosures: G.M. Ghobrial: None. V. Thakkar: None. E. Andrews: None. M.J. Lang: None. A. Chitale: None. M.E. Oppenlander: None C. Maulucci: None. A.D. Sharan: None. J.S. Harrop: None. J. Jallo: None. S. Prasad: None.

Study Design: Retrospective Case Series.

Objective: To demonstrate the microbial trends of spinal surgical site infections(SSI) in patients who had previously received crystallized vancomycin in the operative bed.

Summary of Background Data: Prior large, case control series demonstrate the significant decrease in SSI with the administration of vancomycin in the wound bed.

Methods: A single institution, electronic database search was conducted for all spinal surgery patients who had received prophylactic crystalline vancomycin powder in the wound bed. Patient's with a prior history of wound infection, intrathecal pumps, or spinal stimulators were excluded

Results: 981 consecutive patients (494 male, 487 female, mean age 59.4 years, range 16-95 years) were identified from January 2011 to June 2013. The average dose of vancomycin powder was 1.13 grams(range: $1-6$ grams). 66 patients $(6.71 \%)$ were diagnosed with a SSI of which 51 patients had positive wound cultures (5.2\%). Of the 51 positive cultures the most common organism was Staphylococcus aureus. The average dose of vancomycin was 1.3 grams in the 38 cases where a gram-positive organism was cultured. A number of gram-negative infections were encountered such as Serratia marcescens, Enterobacter aerogenes, Bacteroides fragilis, Enterobacter cloacae, Citrobacter koseri and Pseudomonas aeruginosa. The average dose of vancomycin was 1.2 grams in 23 cases where a gram negative infection was cultured. 15 of the $51(29.4 \%)$ positive-cultures were polymicrobial. 8 (53\%) of these 15 polymicrobial cultures contained three or more distinct organisms.
Conclusions: Prophylactic intraoperative vancomycin use in the wound bed in spinal surgery may increase the incidence of gramnegative or polymicrobial spinal infections. The use of intraoperative vancomycin may correlate with postoperative seromas, due to the high incidence of non-positive cultures. Large, randomized, prospective trials are needed to demonstrate causation and dose-response relationship.

\section{Paper 11. Lumbar Spine Posterior Subcutaneous Fat Depth as a Risk Factor for Surgical Site Infection}

John J. Lee; Khalid I. Odeh, BA; Rakesh D. Patel, MD; James A. Goulet, MD; Gregory P. Graziano, MD

\section{University of Michigan, Orthopaedic Surgery}

Disclosures: J.J. Lee: None. K.I. Odeh: None. R.D. Patel: 2; I; Stryker spine. J.A. Goulet: 1; I; zimmer. 6; I; pioneer surgical technology. G.P. Graziano: 5; I; Medtronic Sofamor Danek.

Introduction: Obesity (BMI $\geq 30)$ is a risk factor for surgical site infections (SSI) in spine procedures. However, BMI does not account for body mass distribution. Subcutaneous fat thickness has been shown to be a risk factor for SSIs in other areas of the body. The purpose of this study was to test the hypothesis that subcutaneous fat thickness in the lumbar spine is a stronger risk factor for SSI than is BMI.

Methods: A retrospective review of 149 adult patients treated at our institution who underwent posterior lumbar surgeries involving midline approaches between 2003-2011 who had CT scans which included the spine and posterior skin. The development of an SSI and previously identified risk factors for SSI (age, diabetes, tobacco use, BMI, anesthesia time, number of levels, prior procedure) were identified using hospital records. Using novel standardized semiautomated analytic morphomic techniques with MATLAB software (Mathworks, Natick, MA), the distance from the spinous process to back skin was obtained at the T12-L5 vertebral levels from CT scans.

Results: Data presented as mean ( \pm standard deviation). The overall rate of SSIs was $9.9 \%(n=15)$. Patients with an SSI had an average age of $51.3( \pm 17.5)$ years, BMI of $32.7( \pm 8.0)$, fat thickness of 59.3 $( \pm 21.3) \mathrm{mm}, 33.3 \%$ had tobacco use, $26.7 \%$ diabetes and $26.7 \%$ underwent prior operations. Patients without an SSI had an average age of $53.7( \pm 15.6)$ years, BMI of $27.9( \pm 6.4)$, fat thickness of $41.5( \pm 16.3) \mathrm{mm}, 30.6 \%$ had tobacco use, $14.9 \%$ diabetes and $9.7 \%$ underwent prior operations. Among factors previously identified to be associated with SSI, bivariate analysis showed BMI ( $\mathrm{p}=0.014)$, obesity $(p=0.015)$, and fat thickness $(p=0.001)$ to be significant. In multivariate logistic regression analysis, BMI and obesity lost their significance and fat thickness was determined to be the only significant risk factor $(\mathrm{p}=0.042)$. For every millimeter of fat thickness there was a $5.5 \%(\mathrm{OR}=1.055,95 \% \mathrm{CI} 1.002-1.111)$ increase in the odds of developing an SSI. When broken down by fat thickness tertiles, those in the highest tertile $(>51 \mathrm{~mm})$ had an 8 -fold increased risk for SSI than the lower tertiles $(\mathrm{p}=0.009)$. Fat thickness and BMI were moderately correlated $(\mathrm{r} 2=0.4783)$.

Conclusions: The thickness of subcutaneous fat is a risk factor for SSI in lumbar spine procedures in our model while BMI/obesity are not. Identifying patients with a critical threshold of fat thickness and targeting treatments for this risk factor may be more economical than identifying patients at risk using BMI. 


\section{Abstracts of the 2014 Meeting of the Lumbar Spine Research Society}

Paper 12. Establishment of a Novel Lung Cancer Spine Metastasis Model in Rodents

Kevin A. Sonn; Sharath S. Bellary, MD; Sohaib Z. Hashmi, BS; John T. Nelson, MD; Chawon Yun, PhD; Amruta Ashtekar, MS; Anjan Ghosh; Michael S. Nickoli, MD; Jason H. Ghodasra, MD; Michael Okoli; Erin L. Hsu, PhD; Wellington K. Hsu, MD

\section{Northwestern University, Orthopaedic Surgery}

Disclosures: K.A. Sonn: None. S.S. Bellary: None. S.Z. Hashmi: None. J.T. Nelson: None. C. Yun: None. A. Ashtekar: None. A. Ghosh: None. M.S. Nickoli: None. J.H. Ghodasra: None. M. Okoli: None. E.L. Hsu: 4; I; Stryker, Zimmer, Medtronic, Pioneer Surgical, Terumo, Spinesmith. 7; I; Medtronic, Pioneer Surgical, Baxter. W.K. Hsu: 4; I; Stryker, Zimmer, Medtronic, Pioneer Surgical, Terumo, Spinesmith. 7; I; Medtronic, Pioneer Surgical, Baxter.

Introduction: Spinal metastases are found in 30-90\% of patients with death attributed to lung cancer. Surgical intervention is often required to stabilize the spine, and these procedures can have significant morbidity that could be avoided with prevention or containment of the metastases by therapeutics. To date, this condition has not been replicated in a pre-clinical animal model, which would greatly aid the study of potential interventions.

Methods: Twenty-seven athymic rats underwent transperitoneal exposure and injection of 50,000 luciferase-labeled A549 lung cancer cells into the L5 vertebral body. At 4 weeks post-implantation, in vivo bioluminescent imaging (BLI), plain anteroposterior radiographs, and microCT imaging were performed to quantitate tumor burden. Histological analysis allowed further characterization of pathologic changes.

Results: BLI showed a vertebral body focal signal in 21/27 animals (Figure 1). Average tumor burden of these rats as determined by BLI radiance measurement was 5.51x104 p/s/cm2/sr. Radiographs and microCT demonstrated osteolysis in $100 \%$ of these 21 animals (Figure 1), and size of osteolysis correlated with BLI signal. Histological analysis also demonstrated tumor invasion causing osteolysis in the L5 vertebral body.

Discussion: This is the first report of a successful lung cancer metastasis model to the lumbar spine. The correlation and consistency of BLI, plain radiographs, microCT, and histological analysis supports this model as a reliable method to study behavior of isolated spinal metastases from lung cancer. This model avoids the pitfalls of systemic tumor burden seen in other metastatic models, such as unpredictable metastases location, while concurrently providing predictable tumor localization that mimics spinal metastasis in humans.

Conclusion: These findings provide a reliable in vivo model to study isolated spinal metastases from primary lung cancer. Subsequent studies will exploit this model to determine whether rhBMP-2 promotes or inhibits the growth and development of A549 lung cancer spine lesions. More generally, this model enables the testing of an array of therapeutic agents as well as possible tumor potentiators, to discern their effects on metastatic lung cancer.

Paper 13. Development of a Novel Lumbar Spinal Cord Injury Model to Examine the Therapeutic Potential of Transplanting Neuronally Induced Neural Stem/Progenitor Cells

Gray Moonen; Charles Tator

University of Toronto

Disclosures: G. Moonen: None. C. Tator: None.

Introduction: Patients with injuries to the thoracolumbar region of the spinal cord often lose neurons essential for locomotion. Our focus is on replacing lost circuitry by transplanting adult spinal-derived

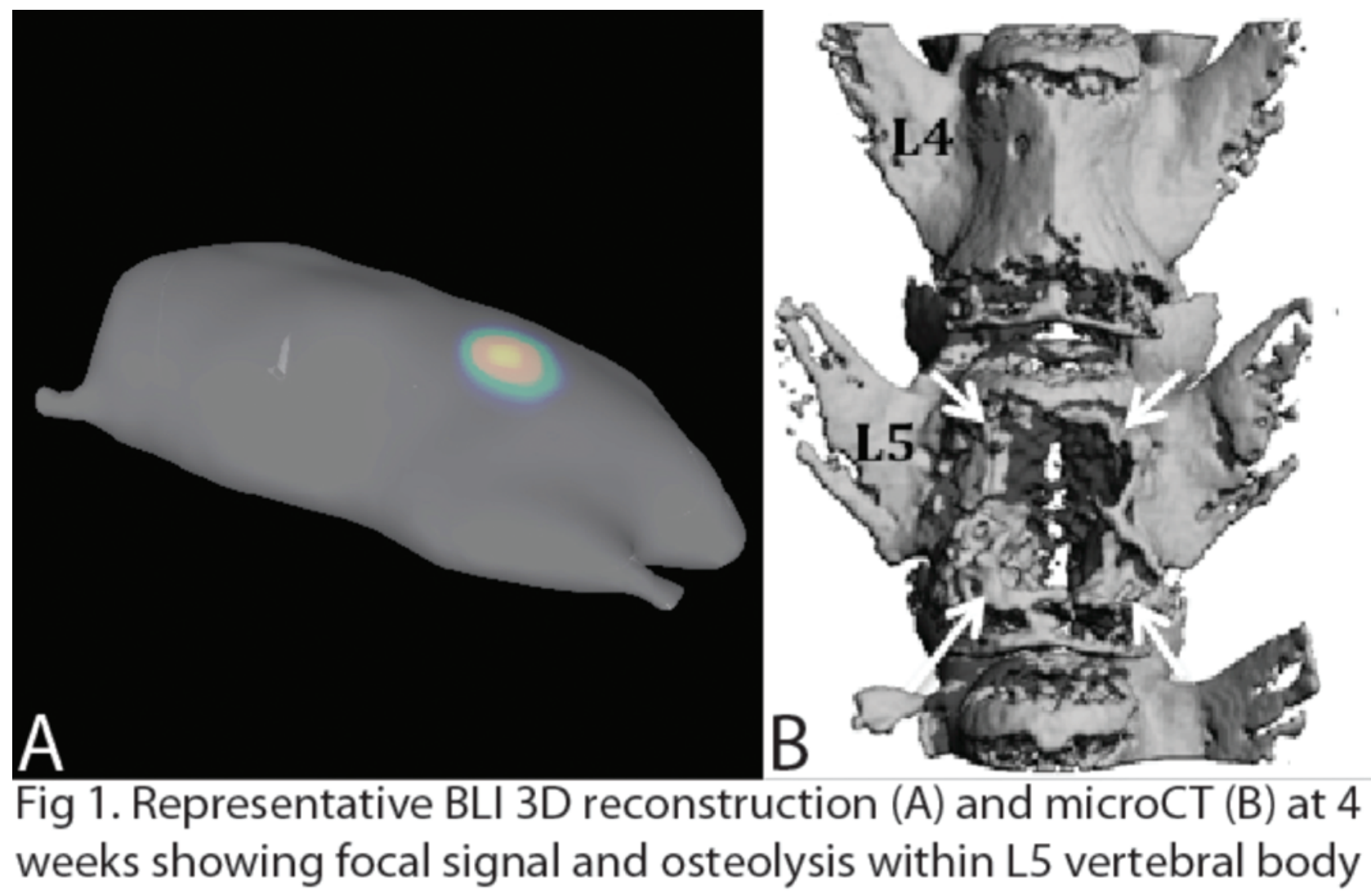




\section{Abstracts of the 2014 Meeting of the Lumbar Spine Research Society}

neural stem/progenitor cells (NSPCs) that have been differentiated into neurons in vitro. We hypothesize that optimal differentiation of NSPCs in vitro towards a neuronal lineage will promote transplant survival and functional recovery after transplantation in the injured lumbar spinal cord.

Methods: NSPCs were treated with $1 \mathrm{mM}$ dibutyryl-cyclic AMP (dbcAMP) to enhance neuronal differentiation and stained with BIII tubulin to confirm neuronal character. To characterize the lumbar spinal cord injury model, various modified aneurysm clips $(56 \mathrm{~g}$, $35 \mathrm{~g}, 26 \mathrm{~g}$ and $20 \mathrm{~g}$ ) were applied to 36 adult female Wistar rats (Wi) to assess spontaneous functional recovery for 6 weeks, as measured by the BBB locomotor scale. $40(\mathrm{Wi})$ rats were injured with a $26 \mathrm{~g}$ clip and split into 4 treatment groups: (1) dbcAMP treated cells + Rolipram injection (RI) post-op, (2) dbcAMP treated cells + saline injection (SI), (3) untreated cells + RI, (4) media injection control + SI. Four hundred thousand cells were transplanted $1 \mathrm{~mm}$ rostral and caudal to the injury site in the subacute phase of injury.

Results: DbcAMP robustly differentiated NSPCs towards BIII positive neurons: $72 \% \pm 6.3$. Rats recovered spontaneously to $2 \pm 1$ in the most severe $56 \mathrm{~g}$ group, $2.5 \pm 1$ in the $35 \mathrm{~g}$ group, $7 \pm 2.5$ in the $26 \mathrm{~g}$ group and $10 \pm 12.5$ in the $20 \mathrm{~g}$ group. In the transplant study, rats in the double treatment group (dbcAMP cells + RI) improved to a statistically significant $(\mathrm{p}<0.05)$ average of $5.16( \pm 3.2)$ compared to dbcAMP + saline $=2.2( \pm 1.5)$, untreated cells $+\mathrm{RI}=1.1( \pm 1.07)$ or control $=2.2( \pm 1.3)$.

Discussion: A possible reason for the exaggerated dysfunction observed in the lumbar spine after injury could be due to the presence of the central pattern generator (CPG). The increase in functional recovery as a result of the neuronal cell transplant + a PDE-IV inhibitor could be due to remodeling of the CPG.

Conclusions: We have generated a novel pre-clinical lumbar spinal cord injury model and displayed that transplant of neuronally differentiated stem cells results in increased functional recovery. aspirates (ICA) and vertebral body aspirates (VBA). The cells were evaluated using four parameters: a nucleated cell count, colony forming unit (CFU) assay, alkaline phosphatase (ALP), and evaluation of the growth factor environment using ELISA for VEGF, PDGF, BMP-2 and BMP-7.

Results: There were 8 men and 7 women with an average age of 66.5 (range 42-80) whom had adherent stromal cellss successfully isolated from all 5 sites. The MBF samples displayed the greatest mean value of isolated cells, however comparison of the median values based on skewed data distribution found no significant differences. CFU assay results suggest more MSCs were isolated from ICBG compared to LLBF and MBF but not ICA nor VBA. Cells from all 5 sites exhibited osteogenic potential based on expression of ALP with ICBG having significantly higher levels than all other sites. Growth factor analysis showed no significant difference in the concentrations of VEGF, PDGF, BMP-2 or BMP-7 between the three bone tissue graft sites (ICBG, LLF, MBF) with one exception (BMP-2 in LLBF vs. ICBF). Those three sites had significantly higher concentrations of all four growth factors compared to the aspirates (Figure 1).

Discussion: This study is the first to rigorously evaluate the cellular and biologic properties of the available graft sites utilized in spine surgery. While the initial goal of this work was to identify one optimal graft site, a combination of local laminectomy bone and vertebral body aspirate may provide a synergistic effect comparable to ICBG
Paper 14. Comparing the Osteogenic Potential of Mesenchymal Stem Cells Isolated from Multiple Lumbar Fusion Bone Graft Sites

Brandon D. Lawrence, MD; Sarina Sinclair, $\mathrm{PhD}$; Darrel S. Brodke, MD

\section{University of Utah, Orthopaedics}

Disclosures: B.D. Lawrence: None. S. Sinclair: None. D.S. Brodke: 1; I; Amedica, DePuy Synthes, Medtronic. 4; I; DePuy Synthes. 6; I; Amedica, Vertiflex.

Introduction: Due to patient dissatisfaction and donor site morbidity associated with iliac crest bone graft (ICBG), many surgeons have come to rely upon the use of local bone graft in an attempt to circumvent these complications. The goal of this study is to compare the osteogenic potential of bone marrow-derived mesenchymal stem cells (BMSCs) isolated from five different autologous bone sites harvested during spine fusion surgery using established in vitro techniques.

Methods: Patients undergoing spinal decompression with instrumented fusion had cells isolated from five different sites: iliac crest bone graft (ICBG), local laminectomy bone fragments (LLBF), morselized bone fragments collected from burr shavings (MBF), iliac crest
Figure 1: The Boxplot of Various Assays by Sites.
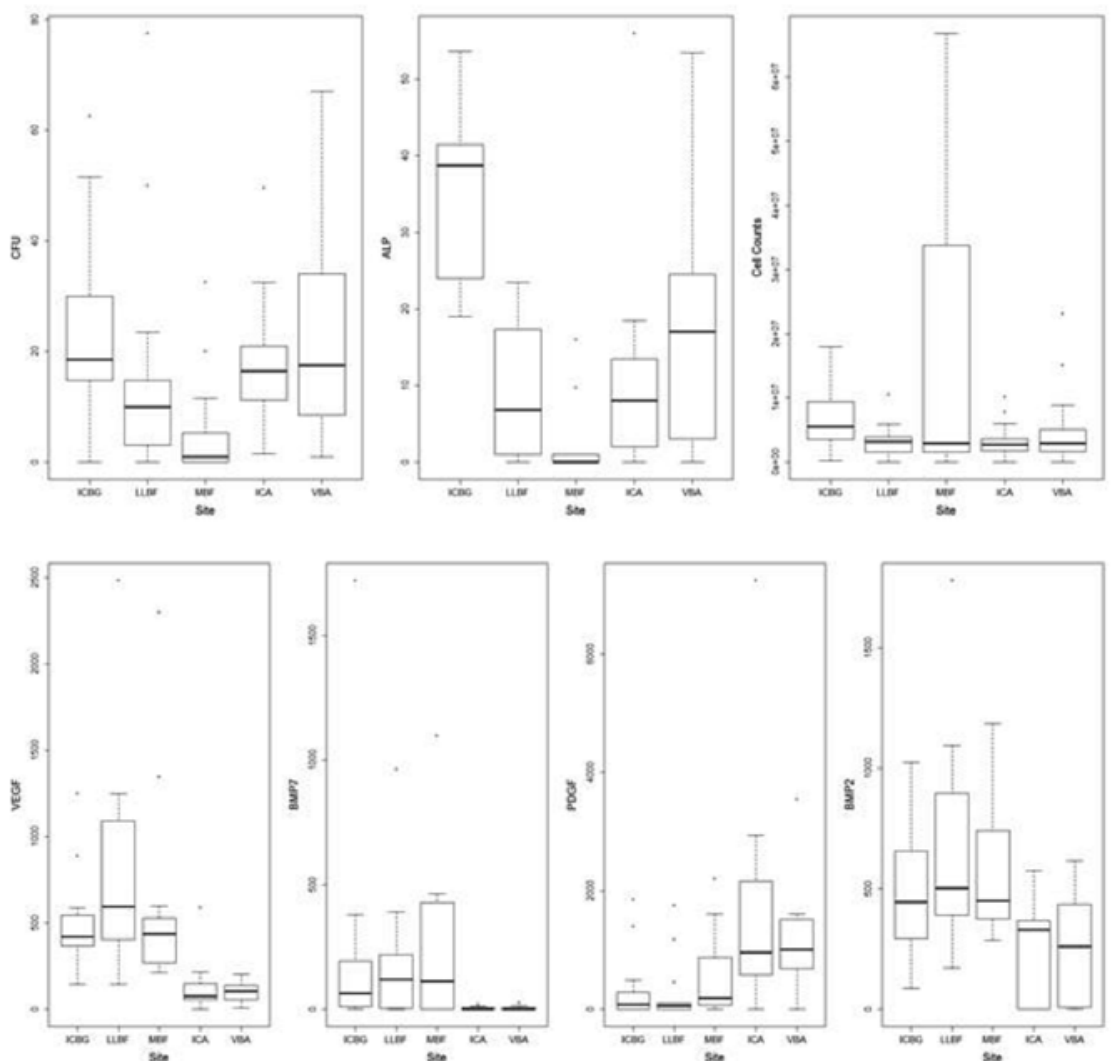


\section{Abstracts of the 2014 Meeting of the Lumbar Spine Research Society}

Paper 15. Effect of Lumbar Intradiscal Injection of Tumor Necrosis Factor-Alpha and Nerve Growth Factor/Vascular Endothelial Growth Factor on Disc Degeneration, Pain Behavior and Neurovascular Ingrowth in an In-Vivo Rat Model

Branko Skovrlj; Alon Lai; Andrew Moon; Devina Purmessur; Beth Winkelstein; Samuel K. Cho; Andrew C. Hecht; James C. Iatridis

Icahn School of Medicine at Mount Sinai, Neurosurgery

Disclosures: B. Skovrlj: None. A. Lai: None. A. Moon: None. D. Purmessur: None. B. Winkelstein: None. S.K. Cho: 4; I; Stryker. A.C. Hecht: 4; I; Zimmer, Medtronic. J.C. Iatridis: None.

Introduction: Low back pain is a common musculoskeletal disorder associated with degenerated intervertebral discs (IVDs). Neurovascular ingrowth has been identified in painful IVDs and it is believed that the irritation of these nerves by the abnormal intradiscal stresses causes pain. The objectives of this study were to explore whether NGF and TNF $\alpha$ induce discogenic pain in association with IVD degeneration and neurovascular ingrowth in an in-vivo rat model.

Methods: 30 skeletally mature Sprague-Dawley rats were randomly divided into five groups: naïve, sham surgery, PBS control, TNF $\alpha$, and NGF/VEGF. The IVDs at L3-4, L4-5 and L5-6 were injected with PBS, TNF $\alpha$ or NGF/VEGF. Pain behavior was assessed for 6 weeks using mechanical hyperalgesia in the hind paw with a series of calibrated von Frey filaments. Function was assessed using the inclined plane test. The severity of IVD degeneration was examined weekly using radiographic disc height measurements. Six weeks after surgery, the rats were euthanized and lumbar spines were collected, decalcified, sectioned and stained.

Results: Average withdrawal was maintained for the naïve and sham groups, decreased immediately post-operatively for the PBS, TNF $\alpha$ and NGF/VEGF groups with mild recovery in the PBS group at one week and continued decrease in the TNF $\alpha$ and NGF/ VEGF groups over time $(\mathrm{p}<0.05)$. Incline test showed poor results in the TNF $\alpha$ and NGF/VEGF groups. IVD heights of naïve and sham groups showed no change. There was a continuous decrease in IVD heights over time in the PBS, TNFa and NGF/VEGF groups $(\mathrm{P}<0.05)$. Intradiscal injection of NGF/VEGF and TNFa induced mild to moderate IVD degeneration, including disorganized annulus fibrosus and decreased number of nucleus pulposus cells.

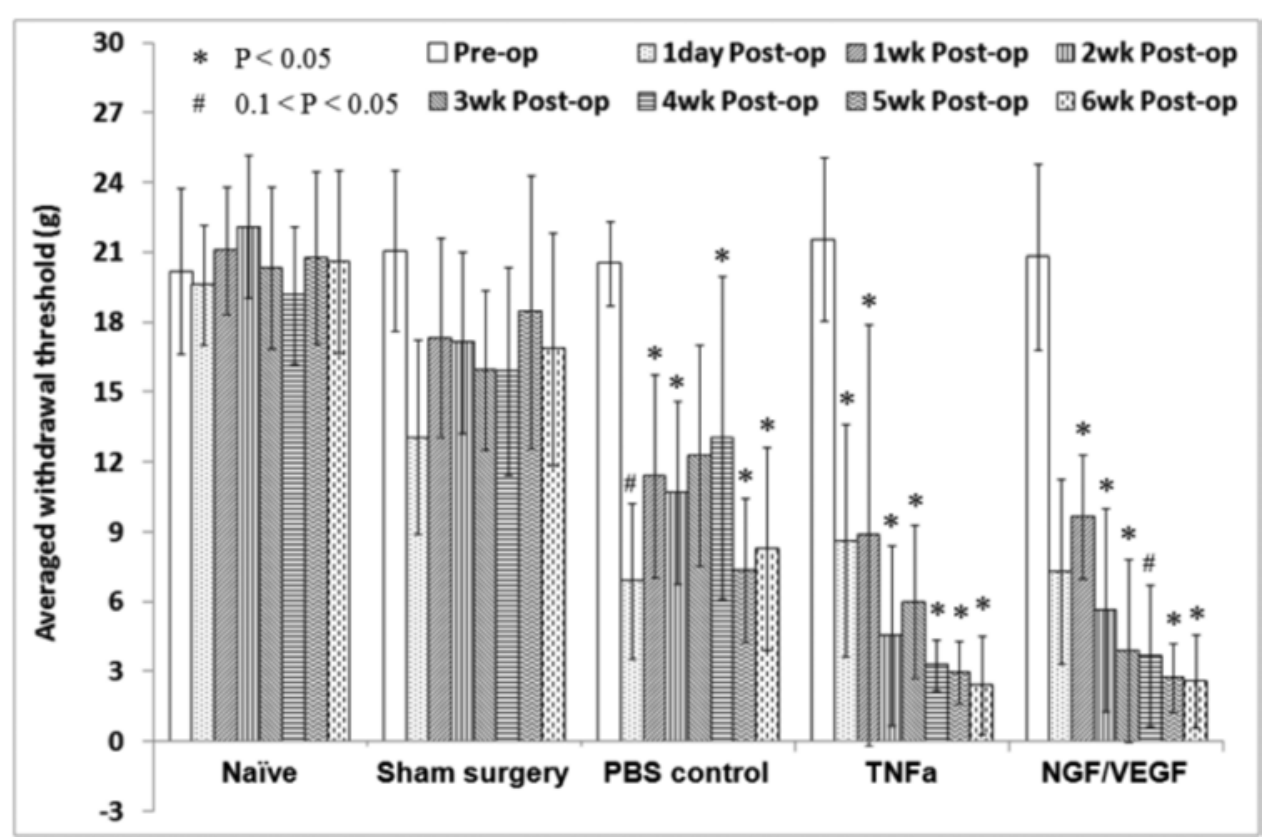

Discussion: Intradiscal injection of TNF $\alpha$ or NGF/VEGF induced functional and histological changes representative of discogenic pain with significantly reduced paw withdrawal thresholds (i.e. pain), decreased performance and loss of IVD height and degeneration. These findings provide insights into the pathophysiology of discogenic back pain and the association between disc degeneration and inflammation.

Conclusions: We present a new discogenic pain model that explores the mechanisms for neurovascular ingrowth and may have high significance for screening future treatment modalities and biological methods of tissue repair.

Paper 16. Comparison of Open and Percutaneous Lumbar Pedicle Screw Revision Rate Using 3-Dimensional Image Guidance and Intraoperative Computed Tomography

Edward Rainier G. Santos, MD; Sharon C. Yson, MD; Jonathan N. Sembrano, MD; David W. Polly, Jr., MD

University of Minnesota, Orthopaedic Surgery

Disclosures: E.G. Santos: 7; I; SI Bone Inc. S.C. Yson: None. J.N. Sembrano: 7; I; NuVasive, Inc.. D.W. Polly, Jr.: None.

Introduction: Complications from malpositioned screws can be devastating and costly. The incidence of neurologic injury secondary to malpositioned screw can be as high as $7-12 \%$. Image-guidance technology has allowed for more precise pedicle screw insertion and intra-operative computed tomography (CT) permitted surgeons to confirm correct placement prior to leaving the operating room. While accuracy of navigated pedicle screw placement in terms of pedicle perforation has been well reported in literature, only a handful of studies examined image-guided pedicle screw accuracy in terms of intra-operative revision and re-operation rates.

Purpose: The aims of this study are to determine intraoperative screw revision and re-operation rates of lumbar pedicle screw inserted with 3-D CT navigation and to compare navigated open and percutaneous techniques.

Methods: We reviewed 199 cases of 3D image-guided lumbar pedicle screw instrumentation from Nov 2006 to Dec 2011. Screw or k-wire removal, repositioning or abandonment of insertion based of intra-operative confirmatory scans were noted. Chi-square was used to determine statistical significance in rates between the two groups $(\mathrm{a}=0.05)$. We also noted for any return to surgery secondary to complications from malpositioned screw.

Results: A total of 988 screws (601 open, 387 percutaneous) were reviewed. Forty-five screws and/or k-wire revisions $(4.6 \%)$ were done overall; 16 screws $(2.7 \%)$ were revised in the open group; $21 \mathrm{k}$-wires $(5.4 \%)$ and 8 screws $(2.1 \%)$ were revised in the percutaneous group (Table). No neurovascular complications secondary to screw position was noted during the immediate post-operative period. No patient needed return to surgery for revision of malpositioned screw. The difference in the revision rates between the open and percutaneous group was statistically significant $(\mathrm{p}=0.0004)$. However, if $\mathrm{k}$-wire revision was excluded and only screw revision was considered, the difference was not statistically significant $(\mathrm{p}=0.55)$. 


\section{Abstracts of the 2014 Meeting of the Lumbar Spine Research Society}

\begin{tabular}{|c|c|c|c|c|c|c|c|c|c|c|c|c|}
\hline & \multicolumn{6}{|c|}{ Open Technique } & \multicolumn{6}{|c|}{ Percutaneous Technique } \\
\hline Lerel & $\begin{array}{l}\text { of } \\
\text { Screws } \\
\text { merted }\end{array}$ & \begin{tabular}{|l|} 
of Screws \\
redirected \\
$(\%)$
\end{tabular} & $\begin{array}{l}\text { - Screws } \\
\text { augmented } \\
\text { by hook (\%) }\end{array}$ & $\begin{array}{l}\text { of } \\
\text { screws } \\
\text { removed } \\
(\%)\end{array}$ & \begin{tabular}{|l} 
of \\
Abandoned \\
screw \\
placement \\
$(\%)$
\end{tabular} & $\begin{array}{l}\text { Revision } \\
\text { rate (\%) }\end{array}$ & $\begin{array}{l}\begin{array}{l}\text { nof } \\
\text { Screws } \\
\text { muserted }\end{array} \\
\end{array}$ & $\begin{array}{l}\text { * of K-wires } \\
\text { redirected } \\
(\%)\end{array}$ & $\begin{array}{l}\text { *of K- } \\
\text { wires } \\
\text { removed } \\
\text { (\%) }\end{array}$ & $\begin{array}{l}\text { of screws } \\
\text { removed } \\
(\%)\end{array}$ & $\begin{array}{l}\text { of Abandoned } \\
\text { sccew placement } \\
\text { \%) }\end{array}$ & $\begin{array}{l}\text { Revision } \\
\text { rate (\%) }\end{array}$ \\
\hline L1 & 24 & $1(4.2)$ & 0 & 0 & 0 & $1(4.2)$ & 14 & 0 & 0 & 0 & 0 & 0 \\
\hline $\mathrm{L}_{2}$ & 37 & $1(2.7)$ & 0 & 0 & $1(2.7)$ & $2(5.4)$ & 35 & $3(8.6)$ & 0 & 0 & $1(2.9)$ & $4(11.4)$ \\
\hline L3 & 79 & $1(1.3)$ & $1(1.3)$ & $1(1.3)$ & 0 & $3(39)$ & 68 & $3(4.5)$ & $2(2.9)$ & 0 & 0 & $5(7.4)$ \\
\hline L4 & 186 & $2(1.1)$ & 0 & $\overline{0}$ & $1(0.5)$ & $3(1.6)$ & 119 & $9(7.6)$ & 0 & $1(0.8)$ & $2(1.7)$ & $12(10.1)$ \\
\hline L5 & 196 & $3(1.5)$ & 0 & $3(1.5)$ & 0 & $6(3.0)$ & 101 & $3(3.0)$ & 0 & 0 & $3(3.0)$ & $6(5.9)$ \\
\hline S1 & 79 & $1(1.3)$ & 0 & 0 & 0 & $1(1.3)$ & 48 & $1(2.1)$ & 0 & 0 & $1(2.1)$ & $2(4.2)$ \\
\hline Total & 601 & $9(1.5)$ & $1(0.2)$ & $4(0.7)$ & $2(0.3)$ & $16(2.7)$ & 387 & $19(49)$ & $2(0.5)$ & $1(0.3)$ & $7(1.8)$ & $29(7.5)$ \\
\hline
\end{tabular}

Table Comparing Open and Percutaneous Technique Intra-operative Revision Rates

Conclusions: We report a $4.6 \%$ intra-operative revision rate with the use of navigation and intra-operative CT scan. This technology has virtually eliminated the need for a re-operation secondary to a malpositioned screw. It may suggest a more cost-effective way of preventing neurovascular injuries and revision surgery. However, considering the potential for increased radiation to patient, more dedicated studies on this new technology's impact on health economics should be undertaken.

\section{Paper 17. Facet Joint Violation During Percutaneous Pedicle} Screw Placement: a Comparison of Two Techniques

Oliver Tannous, MD; Ehsan Jazini, MD; Kelley Banagan, MD; Daniel Gelb, MD; Eugene Koh, MD, PhD; D. Greg Anderson, MD; Steven Ludwig, MD

\section{University of Maryland Medical Center, Orthopaedics}

Disclosures: O. Tannous: None. E. Jazini: None. K. Banagan: 3; I; Spinal Dimensions. D. Gelb: 1; I; Globus Medical, Depuy-Synthes Spine. 2; I; Depuy-Synthes Spine. 4; I; Globus Medical, Depuy-Synthes Spine, Alphatec. 6; I; Advanced Spinal Intellectual Property. E. Koh: 4; I; Biomet. D. Anderson: 1; I; Medtronic; DePuy, A Johnson \& Johnson Company. 2; I; DePuy, A Johnson \& Johnson Company; Medtronic. 4; I; DePuy, A Johnson \& Johnson Company; Medtronic; Synthes, Globus Medical. 7; I; DePuy, A Johnson \& Johnson Company. 9; I; Thieme. S. Ludwig: 1; I; DePuy, A Johnson \& Johnson Company; Globus Medical. 2; I; DePuy, A Johnson \& Johnson Company; Synthes. 4; I; DePuy, A Johnson \& Johnson Company; Globus Medical; Synthes. 6; I; Globus Medical; Alphatec Spine, ASIP, Spinicity, ISD. 7; I; Globus Medical. 9; I; Thieme, QMP.

Background/Purpose: Percutaneous pedicle screw instrumentation is a method of fixation for a variety of spinal disorders. Minimally invasive spinal techniques decrease perioperative morbidity, and possibly adjacent segment disease, which has been associated with iatrogenic damage to the facet joints during open pedicle screw placement. Technical variations of percutaneous pedicle screw placement may pose different risks to the facet joint and progression towards adjacent segment disease. The purpose of this study is to compare the rate of iatrogenic facet joint and pedicle wall violation between two methods of percutaneous pedicle screw instrumentation in the thoracic and lumbar spine.

Methods: Pedicle screws were percutaneously placed in human cadaveric spines by attending spine surgeons from T2 to L5. At each level, screws were instrumented on one side using the 9 or 3 o'clock reference points of the pedicle on the posteroanterior view with a lateral-to-medial trajectory (LMT), and on the contralateral side using the center of the pedicle with an owl's eye trajectory (OET). Postoperative screw placement was evaluated by computed tomography $(\mathrm{CT})$, followed by open anatomic dissection. Outcome measures included facet joint violation and medial pedicle wall breach.
Results: Seven spines were instrumented. Data points were analyzed for thoracic (T2 to T10), thoracolumbar (T11-L1), and lumbar (L2-L5) levels. Overall, 17 of 105 screws placed with a LMT, versus 49 of 105 screws placed with a OET violated or abutted the facet joint $(\mathrm{p}<0.0001)$. This significant difference was observed at the thoracic, thoracolumbar, and lumbar levels ( $\mathrm{p}=0.003,0.035,0.018$ respectively). Medial pedicle wall breaches occurred at 11 MLT screws and 7 OET screws $(\mathrm{p}=0.077)$, none of which were critical breaches. All facet joint violations and medial pedicle breaches were evaluated with postoperative CT and confirmed by open dissection.

Discussion/Conclusions: Significantly more facet joint violations occur using the owl's eye percutaneous pedicle screw technique versus the lateral-to-medial trajectory technique in the thoracic, thoracolumbar, and lumbar spine. No statistically significant differences in medial pedicle wall violation occurred between techniques. Thus when performing minimally invasive pedicle screw fixation, using the lateral-to-medial trajectory may reduce iatrogenic damage to facet joints and the subsequent development of adjacent segment disease.

Paper 18. Increased Incidence of Pseudarthrosis after Unilateral Instrumented Transforaminal Lumbar Interbody Fusion in Patients with Lumbar Spondylosis

Branko Skovrlj; Yakov Gologorsky; Jeremy Steinberger; Max Moore; Frank Moore; Alfred Steinberger; Marc Arginteanu

\section{Icahn School of Medicine at Mount Sinai, Neurosurgery}

Disclosures: B. Skovrlj: None. Y. Gologorsky: None. J. Steinberger: None. M. Moore: None. F. Moore: None. A. Steinberger: None. M. Arginteanu: None.

Introduction: Transforaminal lumbar interbody fusion (TLIF) with segmental pedicular

instrumentation is a well-established procedure employed to treat lumbar spondylosis with or without spondylolisthesis. Available biomechanical and clinical studies comparing unilateral versus bilateral constructs have produced conflicting data regarding patient outcomes and hardware complications.

\begin{tabular}{lccc}
\hline Table 2. Operative Data & & & \\
\hline No. of patients & Unilateral & Bilateral & p-value \\
EBL (cc) & 40 & 40 & \\
LOS (days) & 396.3 & 502.5 & 40.001 \\
Neurologic injury & 3.75 & 3.83 & 0.69 \\
Pseudoarthrosis & $0 / 40$ & $0 / 40$ & 1.00 \\
Cage migration & $7 / 40(17.5 \%)$ & $1 / 40(2.5 \%)$ & 0.05 \\
Screw breakage & $0 / 40$ & $0 / 40$ & 1.00 \\
Wound dehiscence & $0 / 40$ & $0 / 40$ & 1.00 \\
Number of Levels & $1 / 40(2.5 \%)$ & $0 / 40$ & 1.00 \\
I & & & \\
2 & 26 & 17 & 0.07 \\
Specific Levels & 14 & 23 & 0.07 \\
L.2-3 & & & \\
L.3-4 & 0 & 1 & 0.31 \\
L4-5 & 1 & 1 & 1.00 \\
L-S-S1 & 9 & 7 & 0.78 \\
L3-5 & 16 & 8 & 0.09 \\
L-S1 & 2 & 2 & 1.00 \\
& 12 & 21 & 0.07 \\
\hline
\end{tabular}

EBL: Estimated blood loss, LOS: Length of Stay 


\section{Abstracts of the 2014 Meeting of the Lumbar Spine Research Society}

Methods: A prospective randomized cohort study was undertaken by a single group of neurosurgeons. 80 patients were randomized prospectively entered into either bilateral or unilateral pedicle screw instrumentation groups, with 40 enrolled into each group. Demographic data collected from both groups included sex, age, body-mass index, tobacco use, and workman's compensation/litigation status. Operative data included segments operated, number of levels, estimated blood loss, length of stay, and perioperative complications. Long term analysis of hardware malfunction, wound dehiscence, and pseudarthrosis were recorded. All patients had preoperative baseline and six month postoperative Medical Outcomes Short Form-36 (MOS SF-36) health outcomes scores recorded.

Results: Patient follow-up ranged from 37 to 63 months with a mean of 52 months. No patients were lost to follow-up. The patients in the unilateral pedicle screw group were slightly younger than those in the bilateral pedicle screw group (Mean age 42 vs. $47, \mathrm{p}=0.02$ ). Otherwise, there was no significant difference in demographic data between the two groups, the mean number of lumbar levels operated, or distribution of the levels operated. Patients in the bilateral pedicle screw instrumentation group had increased estimated blood loss (EBL), but similar length of stay. There was a significantly increased incidence of pseudarthrosis (7 patients, $17.5 \%$ ) in patients undergoing unilateral instrumentation compared with instrumented bilaterally (1 patient, 2.5\%) ( $\mathrm{p}=0.02)$. There was one wound dehiscence in the unilateral instrumentation cohort. In all, eight patients in the unilateral instrumentation cohort were offered reoperation compared to one in the bilateral group $(\mathrm{p}=0.03)$ All patients had significant improvement in the physical component of the SF-36 $(\mathrm{p}<0.001)$.

Discussion: TLIF with either unilateral or bilateral segmental pedicular instrumentation is an effective treatment for lumbar spondylosis.

Conclusions: TLIF with bilateral constructs versus unilateral constructs may be superior biomechanically, as patients with unilateral constructs were 7 times more likely to suffer from pseudarthrosis and require reoperation.

Paper 19. Predictive factors for APJF after Adult Deformity Surgery with UIV in the Thoracolumbar (TL) Spine

Prokopis Annis, MD; Brandon D. Lawarence, MD; William R. Spiker, MD; Michael D. Daubs, MD; Yue Zhang, PhD; Darrel S. Brodke, MD

\section{University of Utah, Department of Orthopaedics}

Disclosures: P. Annis: None. B.D. Lawarence: None. W.R. Spiker: None. M.D. Daubs: 1; I; Synthes Spine. 4; I; Synthes Spine. Y.Zhang: None. D.S. Brodke: 1; I; Amedica, DePuy Synthes, Medtronic. 4; I; DePuy Synthes. 6; I; Amedica, Vertiflex.

Introduction: Acute Proximal Junctional Failure (APJF), was recently defined as: postoperative fracture of the upper instrumented vertebrae (UIV) or UIV+1; UIV implant failure; proximal junctional kyphosis (PJK) increase $>15^{\circ}$; or need for proximal extension of the fusion within 6 months of surgery. The incidence and revision rates of APJF are thought to be higher when the UIV is located in the lower thoracic or lumbar (TL) spine, and maybe with overcorrection. This study is the first to assess independent predictive factors and timing for revisions of APJF.

Multivariate logistic regression on APJF with age Adjustment

\begin{tabular}{llr}
\hline Variable names & OR $(95 \%$ CI $)$ & P value \\
\hline LL Change $>30^{\circ}$ & $6.94(1.67,28.75)$ & 0.008 \\
Post PJA $>5^{\circ}$ & $27.77(3.18,242.47)$ & 0.003 \\
Pre-postPJA $\Delta>10^{\circ}$ & $7.73(2.12,28.13)$ & 0.002
\end{tabular}

Methods: Retrospective review of 135 consecutive patients with minimum 2-year follow up, treated at a single institution for adult spinal deformity, all with UIV in the TL spine (T9-L2). Fusions were divided into 3 groups based on the UIV location (T9-T10 vs T11-T12 vs L1-L2). Radiographic measurements and demographic data were reviewed. Incidence and failure modes of APJF, as well as timing are reported. Risk factors for APJFI were assessed with univariate and multivariate regression analysis models.

Results: 135 consecutive patients were reviewed, with mean follow-up 42 months (24-126). Mean age was 66 years (24-86). The incidence of APJF was $38.5 \%$, with a trend towards higher APJF failure in the T9-T10 group $(\mathrm{p}=0.07)$. The overall revision rate for APJF was $17 \%$, most, often for UIV fracture. PJK $>15^{\circ}$ was the form of APJF that least often lead to revision (highest survival)(86\%). Univariate analysis revealed preoperative SVA $>5 \mathrm{~cm}$ and postoperative PJA $>5^{\circ}$, thoracic kyphosis $>30^{\circ}$ and instrumentation to pelvis as risk factors for APJF. Multivariate regression analysis confirmed postoperative $\mathrm{PJA}>5^{\circ}$, and greater correction of LL are independent risk factors for APJF.

Conclusions: The incidence of acute proximal junctional failure in adult deformity patients is higher than previous reported if the UIV is in the lower thoracic and lumbar spine. Fracture at the UIV or UIV+1 lead to the highest revision rate, while $\mathrm{PJK}>15^{\circ}$ had the longest revision-free survival. Postoperative $\mathrm{PJA}>5^{\circ}$ and greater correction of LL are independent risk factors for APJF.

Paper 20. The Effect of Lumbopelvic Morphology on Early Lumbar Degenerative Disc Disease in Adolescents

Gregory D. Schroeder; Marco C. Mendoza, M.D.; Erika L. Daley; Cynthia R. LaBella, M.D.; Jason W. Savage, M.D.; Alpesh A. Patel, M.D. FACS; Wellington K. Hsu, M.D.

Northwestern University Feinberg School of Medicine, Orthopaedic Surgery

Disclosures: G.D. Schroeder: None. M.C. Mendoza: None. E.L. Daley: None. C.R. LaBella: 9; I; american academy of pediatrics. J.W. Savage: None. A.A. Patel: 1; I; Amedica, Ulrich Medical USA. 4; I; Amedica, Biomet, DePuy, GE Healthcare, Stryker, Zimmer. 6; I; Amedica, Cytonics, Nocimed, Trinity Orthopaedics. 9; I; Springer. W.K. Hsu: 4; I; AONA, Lifenet, Medtronic, Pioneer Surgical, Stryker, Terumo, Zimmer. 7; I; Baxter.

Introduction: Abnormal lumbopelvic alignment alters the forces through the lumbar spine and is associated with an increased risk of spondylolisthesis. However, it is unknown if the irregular forces increase the risk of developing degenerative disc disease in adolescents.

Methods: Medical records of pediatric patients (ages 10-18)

\begin{tabular}{|c|c|c|c|c|c|}
\hline \multicolumn{6}{|c|}{ Table 1} \\
\hline & $\begin{array}{l}\text { Number of } \\
\text { Patients }\end{array}$ & $\begin{array}{c}\text { Pfirrmann } \\
\text { Average }\end{array}$ & $\begin{array}{c}\% \text { with } \\
\text { abnormal } \\
\text { Pfirmann }\end{array}$ & $\begin{array}{c}\% \text { with } \\
\text { abnormal } \\
\text { HNP }\end{array}$ & $\begin{array}{l}\% \text { with } \\
\text { abnormal } \\
\text { HNP or } \\
\text { Pfirmann }\end{array}$ \\
\hline $\mathrm{PI}>60$ & 10 & 1.04 & $10 \%$ & $20 \%$ & $20 \%$ \\
\hline $\mathrm{PI}<60$ & 43 & 1.14 & $35 \%$ & $35 \%$ & $40 \%$ \\
\hline P Value & & 0.07 & 0.25 & 0.47 & 0.30 \\
\hline $\mathrm{PT}>20$ & 10 & 1.10 & $20 \%$ & $30 \%$ & $30 \%$ \\
\hline PT $<20$ & 43 & 1.13 & $33 \%$ & $33 \%$ & $37 \%$ \\
\hline P Value & & 0.70 & 0.70 & 1.00 & 1.00 \\
\hline $\begin{array}{c}\mathrm{PI} \text { - Lordosis > } \\
9\end{array}$ & 30 & 1.11 & $30 \%$ & $30 \%$ & $33 \%$ \\
\hline $\begin{array}{c}\mathrm{PI}-\text { Lordosis < } \\
9\end{array}$ & 23 & 1.14 & $30 \%$ & $35 \%$ & $39 \%$ \\
\hline$P$ value & & 0.69 & 1.00 & 0.77 & 0.78 \\
\hline
\end{tabular}




\section{Abstracts of the 2014 Meeting of the Lumbar Spine Research Society}

between 2004-2012 were evaluated. Patients with symptomatic low back pain, a lumbar spine MRI, and lateral radiograph demonstrating the femoral heads and the lumbar spine were included. Patients with spondylolysis/spondylolisthesis, a cobb angle $>20$ degrees, a congenital vertebral anomaly, or a medical history affecting spinal development were excluded. Patients with a pelvic incidence $(\mathrm{PI})>$ 60 degrees, a pelvic tilt $(\mathrm{PT})>20$ degrees, or lumbar lordosis-pelvic incidence mismatch (LL not within nine degrees of PI) were considered to have abnormal lumbopelvic alignment and were compared to patients with normal lumbopelvic alignment. Lumbar radiographs and MRIs were randomized, blinded and reviewed by two authors. Each intervertebral disc was assigned a Pfirrmann grade and evaluated for a herniated disc. Decisions were adjudicated by the senior author in cases of disagreement.

Results: Fifty-three patients met the inclusion/exclusion criteria, and $37(70 \%)$ had abnormal lumbopelvic alignment. Ten (19\%) had a PI > 60 degrees, $10(19 \%)$ had a PT > 20 degrees, and $30(57 \%)$ had a LL-PI mismatch. The prevalence of either disc degeneration or herniated discs was similar between patients with abnormal $(30 \%)$ and normal $(50 \%, \mathrm{p}=0.22)$ lumbopelvic alignment. There was no difference in the average Pfirmann grade $(1.10$ versus $1.18, \mathrm{p}=$ $0.37)$, percentage of patients with at least one degenerative disc (10, $(27 \%)$ versus $6,(38 \%), p=0.52)$, or rate of herniated discs $(10,(27 \%)$ versus $7,(44 \%) \mathrm{p}=0.34)$. Patients with an abnormal pelvic incidence, abnormal pelvic tilt or LL-PI mismatch had no difference in incidence of degenerative or herniated discs (Table 1).

Conclusion: Adolescents with low back pain have a high incidence of abnormal lumbopelvic alignment, most commonly a lumbar lordosis-pelvic incidence mismatch; however, patients with low back pain and abnormal lumbopelvic parameters are not at an increased risk of lumbar degenerative changes.

Paper 21. Lumbopelvic Fixation With S2-alar-iliac Screws Or Iliac Bolts: Risk Factors For Reoperation

Marcus D. Mazur, MD; Vijay M. Ravindra, MD; Meic H. Schmidt, MD, MBA; Brandon D. Lawrence, MD; Darrel S. Brodke, MD; Andrew T. Dailey, MD

University of Utah, Neurosurgery

Disclosures: M.D. Mazur: None. V.M. Ravindra: None. M.H Schmidt: None. B.D. Lawrence: None. D.S. Brodke: None. A.T. Dailey: 4; I; Biomet.

Introduction: S2-alar-iliac (S2AI) screws are an attractive alternative to conventional fixation with iliac bolts since they are lower profile, require less muscle dissection, and have greater pullout strength. Few studies, however, compare outcomes between these techniques.

Methods: We conducted a retrospective study of consecutive adult patients at the University of Utah from December 2009 to March 2012 who underwent lumbopelvic fixation using S2AI screws or iliac bolts. Medical records were reviewed for patients who required reoperation due to instrumentation failure and/or wound-related complications. Post-operative imaging was reviewed for instrumentation loosening or breakage. Univariate, multivariate and survival analyses were used to compare patients who required reoperation with those who did not.

Results: Of the 60 patients included, 23 received S2AI screws. Seventeen patients (28\%) underwent an osteotomy. Mean ODI decreased from 49.1 to 38.5 after surgery. Mean follow-up was 22.3 months. Failure-free survival rate was $96.6 \%$ at 6 months, $87.0 \%$ at 1 year, and $73.5 \%$ at 2 years. Reoperation was more common in patients with iliac bolts than those with S2AI screws (13 vs. 2 ; $\mathrm{p}=0.021$ ). Risk factors for reoperation included placement of iliac bolts (OR 16.7, $95 \%$ CI $[2.34,204] ; \mathrm{p}=0.018)$, having a previous failed lumbar fusion (8.6 [2.48, 45.74]; $\mathrm{p}=0.012)$, and length of construct $(1.2[1.04,1.51]$; $\mathrm{p}=0.023)$. Undergoing an osteotomy, smoking and use of BMP were
Survival function estimates for S2AI screws and iliac bolts

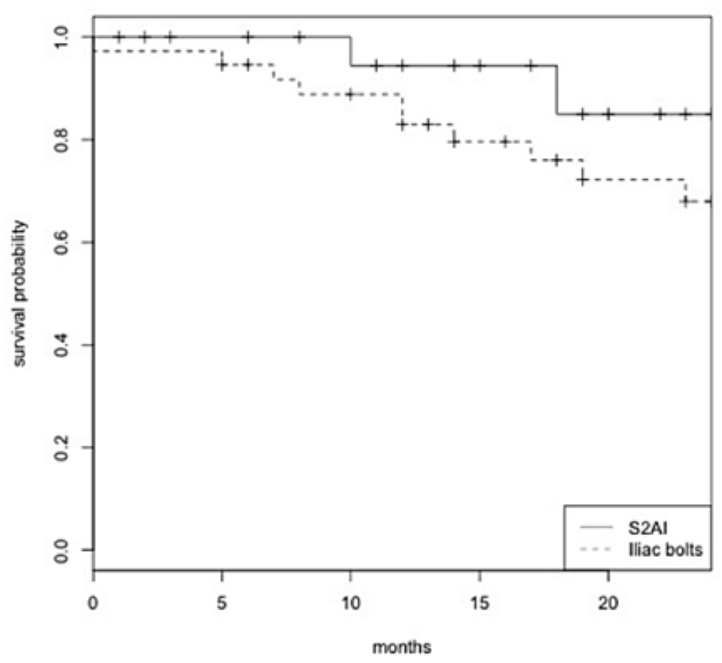

not associated with surgical outcomes. Improvement in ODI was not associated with method of pelvic fixation.

Discussion: Subanalysis revealed that reoperation for wound breakdown, removal of iliac fixation or L5-S1 nonunion occurred in 6 patients with iliac bolts and 1 with S2AI screws $(p=0.16)$. Eight patients required reoperation for instrumentation failure or nonunion between L1 and L5. Six additional patients had fractured instrumentation but did not require reoperation.

Conclusions: High rates of fusion at L5-S1 can be achieved in patients undergoing lumbopelvic fixation with either S2AI screws or iliac bolts. Early results suggest that patients with S2AI screws have a trend towards lower failure rates and fewer reoperations than those with iliac bolts.

Paper 22. Sacral Screw Strain in a Long Posterior Spinal Fusion Construct with Sacral Alar-Iliac (S2AI) versus Iliac Fixation

Daniel G. Kang, MD; Ronald A. Lehman, Jr., MD; Robert W. Tracey, MD; Rachel E. Gaume, BS; John P. Cody, MD; Khaled M. Kebaish, MD; Lawrence G. Lenke, MD

Walter Reed National Military Medical Center, Orthopaedic Surgery

Disclosures: D.G. Kang: None. R.A. Lehman, Jr.: 7; I; Depuy Synthes Spine, Centinel Spine. R.W. Tracey: None. R.E. Gaume: None. J.P. Cody: None. K.M. Kebaish: 4; I; K2M, Depuy Synthes Spine, Orthofix. 5; I; Trans1. 6; I; K2M. 7; I; Depuy Synthes Spine. 8; I; Depuy Synthes Spine. L.G. Lenke: 1; I; Medtronic. 7; I; Axial Biotech, Depuy Synthes Spine. 9; I; Quality Medical Publishing.

Background: Long instrumented posterior fusion constructs to the lumbosacral spine have a significant rate of pseudoarthrosis and S1 screw failure. With the increasing popularity of Sacral Alar-Iliac (S2AI) fixation with its purported advantages of 1) decreased implant profile and 2) obviating the need for a lateral offset connector, the biomechanical properties with respect to $\mathrm{S} 1$ screw strain remain unknown. We set out to compare the biomechanical effect of S2AI versus traditional iliac screw fixation on $\mathrm{S} 1$ screw strain.

Methods: Five fresh-frozen human cadaveric specimens were instrumented from L2-pelvis, maintaining all osteoligamentous structures, with bilateral titanium $6.0 \times 40-\mathrm{mm}$ pedicle screws and $5.5-\mathrm{mm}$ cobalt-chromium rods. Bilateral S1 pedicles were instrumented with $8.0 \times 50-\mathrm{mm}$ screws that were centrally cored out and two uniaxial strain gauges inserted at $0^{\circ}$ and $90^{\circ}$. S2AI and/or Iliac fixation with 


\section{Abstracts of the 2014 Meeting of the Lumbar Spine Research Society}

8.0x80-mm titanium pedicle screws was performed to evaluate four different constructs: (1) Bilateral S1 Screws (control); (2) Bilateral S2AI; (3) Bilateral Iliac; (4) Hybrid (S2AI with contralateral Iliac). Bilateral S1 screw strain was measured (microstrain), and pure moment loads $(12.0 \mathrm{Nm})$ were applied in axial rotation $(\mathrm{AR})$, flexionextension (FE) and lateral bending (LB).

Results: Compared to S1 screws alone, both S2AI and Iliac fixation significantly reduced sacral screw strain in FE by $58 \%$ and $67 \%$, respectively $(\mathrm{p}<0.05)$, in AR by $35 \%$ and $41 \%$, respectively $(\mathrm{p}<0.05)$, while no significant difference in LB for either construct ( $p>0.05$ ). Hybrid constructs demonstrated a significant reduction in only FE, with reduction in screw strain by $56 \%$ for S2AI and 59\% for Iliac fixation, with no difference in AR and LB moments. When S2AI and Iliac fixation were compared, there was no significant difference in screw strain for all bending moments ( $p>0.05)$. Similarly, hybrid constructs demonstrated no side-to-side significant difference between S2AI and Iliac fixation for all bending moments $(\mathrm{p}>0.05)$.

Conclusions: Both S2AI and Iliac fixation provide significant reduction in S1 sacral screw strain compared to sacral fixation alone. Bilateral S2AI fixation is a viable and biomechanically comparable alternative to traditional Iliac fixation, and presents another option to achieve protection of the S1 sacral screws for long segment constructs to the pelvis.

\section{Paper 23. The Effect of Vitamin D Deficiency on Spinal Fusion in an Aged Rat Model Using BMP2}

Michael D. Daubs, MD; Kevin H. Phan; Chengjie Xiong; Tetsuo Hayashi; Raed Alobaidaan; Haijun Tian; Trevor P. Scott; Chelsea B. Fan; Jeffrey C. Wang

\section{UCLA, Orthopaedic Surgery}

Disclosures: M.D. Daubs: 1; I; Depuy-Synthes. 4; I; Depuy-Synthes. 7; I; Stryker. K.H. Phan: None. C. Xiong: None. T. Hayashi: None. R. Alobaidaan: None. H. Tian: None. T.P. Scott: None. C.B. Fan: None. J.C. Wang: 1; I; Biomet, Stryker, Medtronics, Depuy-Synthes, Amedica, Osprey, Aesculap, Seaspine. 6; I; Fziomed, Promethean Spine, Paradigm Spine, Benevenue, NexGen, Pioneer, Amedica, Vertiflex, Electrocore, Surgitech, Axiomed, VG Innovations, Corespine, Expanding Orthopaedics, Syndicom, Osprey, Bone Biologics, Curative Biosciences, Pearldiver.

Introduction: Vitamin D deficiency is one of the most common deficiencies in our population, especially in older patients. Vitamin D plays a crucial role in maintaining bone mineral density and bone strength; however, little is known about how Vitamin D levels affect spinal fusion rates. The purpose of our study was to evaluate how Vitamin D levels affect posterolateral fusion in aged rats.

Methods: Twenty-seven male, 7-week old and twenty-four male, 17-month old Fischer 344 rats were fed a Vitamin D deficient (VDD) diet and housed in a UVB-deficient environment for 6 weeks. After 6 weeks, Vitamin D deficiency was confirmed via ELISA, and bilateral posterolateral intertransverse lumbar fusion at L4-5 was performed using a standard midline approach. BMP2 1ug on an absorbable collagen sponge was used on all animals. After surgery, each of the age groups was divided into 3 groups: VDD diet, normal diet (1 IU/g), and supplemental Vitamin D (SSVD) diet (10 IU/g). Vitamin D levels were confirmed at 4 and 8 weeks post-operation via ELISA. Fusion was assessed by Xray at 2, 4, 6, and 8 weeks and by manual palpation at sacrifice.

Results: At 2 weeks post-surgery, fusion rates for young/aged rats assessed by Xray were: VDD diet; $56 \% / 0 \%(\mathrm{p}<0.05)$; normal 56\%/10\% $(\mathrm{p}=0.04)$; SVD $44 \% / 0 \%(\mathrm{p}<0.05)$. At 4 weeks: VDD 72\%/25\% ( $\mathrm{p}=0.02)$; normal 61\%/62.5\% ( $>0.05)$; and SVD $61 \% / 57 \%(\mathrm{p}>0.05)$. At 6 weeks: VDD 83\%/33\% ( $=0.01)$; normal $67 \% / 75 \%(\mathrm{p}>0.05) ;$ SVD $67 \% / 50 \%(\mathrm{p}>0.05)$. At 8 weeks: VDD $89 \% / 50 \%(\mathrm{p}=0.03)$; normal $83 \% / 83 \% \quad(\mathrm{p}>0.05)$; SVD $72 \% / 67 \%$ $(\mathrm{p}>0.05)$. Radiographically, there was no significant difference between the different diets within each age group at all time points. After sacrifice at 8 weeks, fusion assessed by manual palpation, young/aged, was: VDD 100\%/67\% ( $\mathrm{p}=0.14)$; normal 100\%/100\% (p>0.05); SVD 78\%/67\% (p>0.05).

Discussion: Vitamin D deficiency did not affect fusion healing rates in young or aged rats. The development of the fusion mass, as visualized radiographically, was delayed in the VDD aged rats compared to the VDD young rats at each time period.

Conclusion: There were no differences in fusion rates between young and aged VDD rats as assessed by manual palpation.

Paper 24. In Situ Tissue Engineering Approach to Spine Fusion via Stem Cell Mobilization and Chemokine-Directed Migration

Anton Zaryanov, DO; Ted Koreckij, MD; Tristan Maerz, MS; Meagan Salisbury, B.S.; Jeff Fischgrund, MD; Daniel K. Park, MD; Kevin Baker

\section{Beaumont Health System, Orthopaedic Research}

Disclosures: A. Zaryanov: None. T. Koreckij: None. T. Maerz: None. M. Salisbury: None. J. Fischgrund: 1; I; Stryker. 4; I; Stryker, Relievant, Fziomed, Baxter. D.K. Park: 4; I; Stryker, Depuy. 6; I; Depuy. K. Baker: 7; I; Globus Medical. 


\section{Abstracts of the 2014 Meeting of the Lumbar Spine Research Society}

Introduction: Marrow-derived stem and progenitor cell populations have been shown to mobilize into peripheral blood in response to significant bodily injury. Once mobilized, the cells migrate to injured tissues overexpressing chemokines, such as stromal cellderived factor-1alpha (SDF-1a). After transmigrating into the injured tissue, the stem cells are able to affect tissue repair and regeneration. We hypothesize that this endogenous response can be harnessed to enhance bone formation in the setting of spine fusion.

Methods: Using cryo-lyophilization, cylindrical Chitosanhydroxyapatite (Ch-HA) scaffolds with an open porous architecture were created. Following the rinsing to remove acetic acid, scaffolds were re-lyophilized for storage. Prior to implantation, scaffolds were incubated in a solution of $100 \mathrm{ng}$ of SDF-1a for 24 hours, while controls were incubated in saline. Female Lewis rats (20) were randomized to one of four treatment groups (Figure 1) andunderwent singlelevel posterolateral lumbar fusion at L4-L5. Following decortication of the transverse processes, one cylindrical Chitosan-HA scaffold was placed on each side of the spine. Animals randomized to Group 1 , or Group 3 underwent three days of subcutaneous injections of AMD-3100 (5 [[Unsupported Character - Symbol Font \&\#61549;]]g/ $\mathrm{kg}$ ) to mobilize marrow-derived stem cells into circulation. Animals in Groups 2 and 4 received subcutaneous injections of saline. At six weeks post-op, spines were imaged with micro computed tomography (uCT) to quantify new bone volume. L4-L5 levels were harvested and bisected in the sagittal plane and fixed in $10 \%$ buffered formalin. Fixed motion segments underwent decalcified histological processing, with the left side sectioned in the coronal plane and the right side sectioned in the sagittal plane. H\&E-stained sections were used for histomorphometric analyses.

Results: The cryo-lyophilization process resulted in the formation of Ch-HA scaffolds with a high degree of porosity and $20 \mathrm{wt} \%$ of osteoconductive coralline hydroxyapatite. Loading of SDF-1a into the Ch-HA scaffolds using a simple incubation technique was successful and efficient. Post-operative rats are currently awaiting endpoints (February) for characterization of new bone formation and fusion success.

Conclusions: Mobilization of marrow-derived stem cells (AMD3100) and chemokine-directed homing using SDF-1a-loaded Chitosan-Hydroxyapatite scaffolds may be a promising method to use stem cells to enhance spine fusion.

\section{Paper 25. Comparison of a Novel Oxysterol Molecule and rhBMP2 Fusion Rates in a Rabbit Posterolateral Lumbar Spine Model}

Trevor Scott, MD; Kevin H. Phan, BS; Haijun Tian, MD; Akinobu Suzuki, MD, PhD; Scott R. Montgomery, MD; Elisa Atti, PhD; Sotirios Tetratis, DDS, PhD; Renata C. Pereira, PhD; Jeffrey C. Wang, MD; Michael D. Daubs, MD; Farhad Parhami, PhD, MBA

\section{UCLA, Department of Orthopedics}

Disclosures: T. Scott: None. K.H. Phan: None. H. Tian: None. A. Suzuki: None. S.R. Montgomery: None. E. Atti: None. S. Tetratis: None. R.C. Pereira: None. J.C. Wang: 1; I; Biomet, Stryker, Medtronics, Depuy-Synthes, Amedica, Osprey, Aesculap, Seaspine. 6; I; Fziomed, Promethean spine, Paradigm Spine, Benevenue, NexGen, Pioneer, Amedica, Vertiflex, Electrocore, Surgitech, Axiomed, VG Innovations, Corespine, Expanding Orthopaedics, Syndicom, Osprey, Bone Bi. M.D. Daubs: 1; I; Depuy-Synthes. 4; I; Depuy-Synthes. F. Parhami: 3; I; Founder of maxBIOpharma which licensed Oxy133 from UCLA.

The non-union rate following lumbar spinal fusion is potentially as high as $25 \%$. Bone morphogenetic protein-2 (rhBMP2) has been used as a biological adjunct to promote bony fusion. However, recently there have been increasing concerns about BMP. Oxysterol
133 (Oxy133) has been shown to promote excellent fusion rates in rodent lumbar spine models and offers a potential alternative to rhBMP2. The purpose of this study is to compare the fusion rate of rhBMP2 and Oxy133 in a randomized control trial using a posterolateral lumbar rabbit spinal fusion model. 24 male adult white New Zealand rabbits $(3-3.5 \mathrm{~kg})$ underwent bilateral posterolateral lumbar spinal fusion at L4-L5. Rabbits were divided into 4 groups: control (A), 30 ug rhBMP2 (B), $20 \mathrm{mg}$ Oxy133 (C), and $60 \mathrm{mg}$ Oxy133 (D). At 4 weeks fusion was evaluated by fluoroscopy, and at 8 weeks the rabbits were sacrificed and fusion was evaluated radiographicalLy, by manual palpation, and with microCT.At 8 weeks fusion by radiographic analysis were: group A $40.0 \%$, group B $91.7 \%$, group C $91.7 \%$, and group D 100\%. At 8 weeks all experimental groups were significantly greater than control. When the fusion masses were evaluated by manual palpation after sacrifice, group A was $0 \%$ fused, group B was $83.3 \%$ fused, group C was $83.3 \%$ fused, and group D was $90 \%$ fused. Analysis of microCT data showed fusion rates as follows: A was $0 \%$ fused, B had $67.7 \%$ fusion, $\mathrm{C}$ had $91.7 \%$ fusion and group $\mathrm{D}$ fusion rate was $80 \%$; all of which were significantly greater than control $(p<0.01)$. Tissue volume of the fusion mass was $901.5 \mathrm{~mm}^{\wedge} 3$ for group A, $1651.6 \mathrm{~mm}^{\wedge} 3$ for group B, $1943.9 \mathrm{~mm}^{\wedge} 3$ for group C, and $2149.6 \mathrm{~mm}^{\wedge} 3$ for group $\mathrm{D}$, again all groups were greater than the control $(p<0.001)$. These findings in a rabbit model demonstrate that both low and high dose $20 \mathrm{mg}$ Oxy133 appears to promote fusion that is equivalent to than $30 \mathrm{ug}$ rhBMP2 and significantly greater than the control group.

Paper 26. Efficacy of BMP2 for the Treatment of Lumbar Pseudarthrosis in a Rodent Spine Model

Jing Li; Michael D. Daubs, MD; Kevin H. Phan; Tetsuo Hayashi; Akinobu Suzuki; Haijun Tian; Trevor P. Scott, MD; Jeffrey C. Wang, MD

\section{UCLA, Orthopaedic Surgery}

Disclosures: J. Li: None. M.D. Daubs: 1; I; Depuy-Synthes. 4; I; Depuy-Synthes. 7; I; Stryker. K.H. Phan: None. T. Hayashi: None. A. Suzuki: None. H. Tian: None. T.P. Scott: None. J.C. Wang: 1; I; Biomet, Stryker, Medtronics, Depuy-Synthes, Amedica, Osprey, Aesculap, Seaspine. 6; I; Fziomed, Promethean Spine, Paradigm Spine, Benevenue, NexGen, Pioneer, Amedica, Vertiflex, Electrocore, Surgitech, Axiomed, VG Innovations, Corespine, Expanding Orthopaedics, Syndicom, Osprey, Bone Biologics, Curative Biosciences, Pearldiver.

Introduction: The rate of pseudarthrosis following lumbar fusion is reported to be as high as $35 \%$. In attempts to improve the fusion rates following pseudarthrosis repair, surgeons have turned to bone growthstimulating biologics, such as bone morphogenetic protein (BMP2). Despite the frequent use of BMP2 in treating pseudarthrosis, there are very few studies showing its efficacy. The purpose of our study is to evaluate the effectiveness of the use of BMP2 in the setting of a pseudarthrosis.

Methods: 17 male Charles Lewis rats underwent L4-5 posterior intertransverse fusion using fresh rat allograft harvested from the same rat strain. At 8 weeks, revision surgery was performed and the fusion mass was explored. 16 rats that had confirmed nonunions were then divided into 2 groups (A: BMP2 1ug; B: BMP2 3ug) and underwent pseudarthrosis repair. The BMP2 solutions were placed on an absorbable collagen sponge (ACS) and placed onto the site of pseudarthrosis. A control group of 8 male Charles Lewis rats underwent a primary L4-5 posterior intertransverse fusion. The fusion masses were assessed at final sacrifice with radiographs and manual palpation (8 weeks).

Results: Rats in group A (BMP2 1ug) had a $87.5 \%$ successful fusion rate and Group B (BMP2 3ug) had a fusion rate of 


\section{Abstracts of the 2014 Meeting of the Lumbar Spine Research Society}

$100 \%$ following pseudarthrosis repair evaluated by manual palpation $(\mathrm{p}=1.000)$. The fusion rate in the control group that had undergone a primary lumbar fusion with BMP 1ug was $100 \%$.

Discussion: When compared to the primary fusion cases using BMP, a higher dose of BMP2 appears to be necessary when attempting to obtain a successful fusion with an established pseudarthrosis.

Conclusions: In the setting of an established posterior lumbar pseudarthrosis, BMP is effective in obtaining a successful fusion.

Paper 27. Use of the Spine AdVerse Events Severity (SAVES) System to Categorize and Report Adverse Events in Spine Surgery.

Peter J. Wagner, MD, MMS; Tamir Ailon, MD; Scott Pascal, BS; Hanbing Zhou, MD; Maribeth Harrigan; Michael Stauff, MD; Anthony Lapinsky, MD; Patrick Connolly, MD; Christian DiPaola, MD

\section{University of Massachussets, Orthopedics}

Disclosures: P.J. Wagner: None. T. Ailon: None. S. Pascal: None. H. Zhou: None. M. Harrigan: None. M. Stauff: None. A. Lapinsky: None. P. Connolly: None. C. DiPaola: None.

Objectives: Analysis of adverse events (AEs) in spine surgery has historically been retrospective, utilizing hospital administrative data. Street et al prospectively identified a higher rate of postoperative complications using the Spine AdVerse Events Severity (SAVES V2) system. Our objective was to determine the incidence, severity and effect on hospital length of stay (LOS) for AEs in spine surgery.

Method: AEs for spine patients were prospectively collected for 18 months and correlated with retrospective data from operative reports. Statistical analyses compared patient demographics, diagnoses, and surgical characteristics to hospital length of stay and likelihood of adverse events.

Results: This system captured 75\% (765/977) of surgical cases for all indications over the study period. 73\% (541/743) of patients experienced at least one $\mathrm{AE}$, with an average of $1.2 \mathrm{AEs}$ per patient (range $0-5)$. The most common AEs were pain control (31\%), urinary retention $(9.7 \%)$, wound infection (6.3\%), and incidental durotomy (5.8\%). For patients experiencing at least one $\mathrm{AE}, 30 \%$ had no effect on LOS, $48 \%$ increased LOS by $1-2$ days, $15 \%$ increased LOS by 3-7 days, and 7\% had prolonged LOS greater than 8 days. Our system captured $25.4 \%$ more adverse events $(60.0 \%$ vs. $34.6 \%$ ) than hospital administrative data. Univariate analysis revealed patient age, emergent surgery, diagnostic and surgical categories, and spine region to be predictors of both AEs and LOS. Instrumentation was predictive of increased LOS but not AEs. The type of AE was strongly associated with LOS. Multivariable analysis of AE likelihood demonstrated emergent surgery to be the strongest independent predictor with an adjusted odds ratio of 8.5 versus elective surgery.

Conclusions: Spine surgery is associated with a high incidence of adverse events, which often prolong hospital length of stay. Better characterization of adverse events and their predictors could lead to improved management strategies that reduce patient morbidity and mortality.
Paper 28. Are Administrative Databases Valid for Use in Academic Research? A Study in Obesity

Nicholas S. Golinvaux, BA; Daniel D. Bohl, MPH; Bryce A. Basques, BS; Michael C. Fu, BS; Elizabeth C. Gardner, MD; Jonathan N. Grauer, MD

\section{Yale University School of Medicine, Department of Orthopaedics}

Disclosures: N.S. Golinvaux: None. D.D. Bohl: None. B.A. Basques: None. M.C. Fu: None. E.C. Gardner: None. J.N. Grauer: 2; I; Stryker. 4; I; Affinergy, Alphatec, Bioventus, Depuy, Harvard Clinical Reserach Institute, Powered Research, Stryker, Transgenomic.

Introduction: Recently, national inpatient database use in orthopaedic research has risen sharply. Specifically, lumbar spine research has seen a substantial increase in these studies, as database sample sizes allow for large-scale analyses of perioperative risk factors and surgical outcomes. The results of these studies now play a significant role in the diagnosis and treatment decisions of orthopaedic surgeons. Unfortunately, without firsthand knowledge of each major database, it can be difficult to judge whether a study presents valid results to the specific questions being asked. Many limitations to using databases exist, including a rising concern over the ability of administrative claims data, such as ICD-9 codes, to accurately determine comorbidity rates. The present study examines these limitations using the easily quantifiable and objective variable of obesity to definitively clarify the relationship between ICD-9 codes and clinical reality.

Methods: We conducted a retrospective cohort study of obesity rates in a sixteen-day inpatient census from a large tertiary hospital. For each patient, ICD-9 diagnosis codes and chart-documented body mass index (BMI) were directly compared.

Results: 2,075 patients were included in the study. Of 573 patients deemed "obese" with a calculated BMI between 30.0-39.9, only 109 patients received the correct ICD-9 code (278.00), giving this code a sensitivity of 0.19 . Of 174 patients deemed "morbidly obese" with a calculated BMI above 40,84 patients received the correct ICD- 9 code (278.01), giving this code a sensitivity of 0.48 .

Discussion: This study highlights obesity as an example of the potential errors inherent to using ICD-9-coded databases for research. ICD-9 codes correctly identified just $19 \%$ of obese patients, an inclusion rate that would be unacceptable in any other study design. This selection bias has the potential to significantly sway the outcomes of studies using ICD-9-coded databases. We further postulate that obesi-

\section{Distribution of ICD-9 Codes within Each BMI Decile}

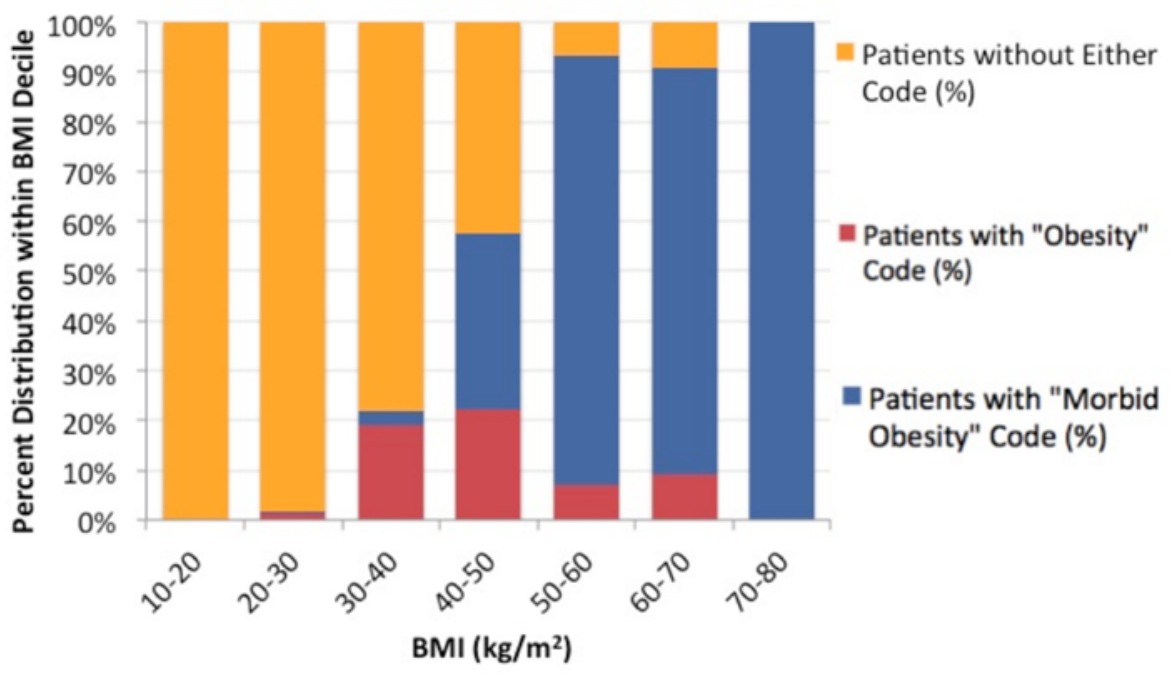




\section{Abstracts of the 2014 Meeting of the Lumbar Spine Research Society}

ty is likely not the only comorbidity for which these results hold true Conclusions: As database research constitutes an increasing proportion of newly published studies, the orthopaedic and spine surgery communities must realize that results can be significantly skewed by database source data, and thus should not be blindly accepted simply by virtue of large sample sizes.

Paper 29. Variability In Spine Surgery Procedures Performed During Orthopaedic And Neurological Surgery Residency Training: An Analysis Of ACGME Case Log Data

Alan H. Daniels, M.D.; Christopher P. Ames, M.D.; John M. DePasse; Justin S. Smith, M.D./Ph.D.; Robert A. Hart, M.D.

\section{Brown University, Orthopaedic Surgery}

Disclosures: A.H. Daniels: 8; I; Stryker. C.P. Ames: 1; I; Lanx; Stryker; Aesculap/B.Braun. 4; I; Medtronic; Stryker; DePuy. 6; I; Trans1; Visualase, Doctors Research Group. 7; I; Trans1. J.M. DePasse: None. J.S. Smith: 2; I; Biomet; Medtronic; Sofamor Danek; Globus Medical; DePuy. 4; I; Biomet; DePuy; A Johnson \& Johnson Company; Medtronic; Sofamor Danek; Globus Medical. 7; I; DePuy. R.A. Hart: 1; I; DePuy; A Johnson \& Johnson Company; SeaSpine. 2; I; DePuy; Kyphon Inc.; Medtronic; Synthes. 4; I; DePuy; Eli Lilly; Medtronic. 6; I; Spine Connect. 7; I; DePuy; Medtronic; OREF; Synthes.

Background: Current spine surgeon training in the United States consists of either an orthopaedic surgery or neurological surgery residency, followed by an optional spine surgery fellowship. Spine surgery exposure may vary between and within specialties.

Methods: Accreditation Council for Graduate Medical Education (ACGME) surgical case logs for orthopaedic surgery and neurological surgery graduating residents from 2008-2012 were examined for spine surgery experience. Variability between and within specialties was compared with a statistical cutoff of $\mathrm{p}<0.05$ for significance.

Results: Graduating neurological surgery trainees report over twice the number of spine surgery cases compared to orthopaedic surgery trainees on average $(\mathrm{p}=0.004)$, although orthopaedic surgery residents have shown an increase in reported total spine procedures each year from 2009-2012 ( $\mathrm{r}=0.961 ; \mathrm{p}=0.039)$. There is substantial variability in spine surgery exposure within both specialties: for spinal instrumentation procedures, there was an 11.6-fold difference between the top and bottom $10 \%$ of orthopaedic residents, and an 8.3-fold difference between the top and bottom $10 \%$ of neurological surgery residents graduating in 2012. For spinal decompression procedures, there was an 8.8 -fold difference between the top and bottom $10 \%$ of orthopaedic residents, and a 4.4-fold difference between the top and bottom $10 \%$ of neurological surgery residents graduating in 2012. For total spine procedures, there was a 5.4-fold difference between the top and bottom $10 \%$ of orthopaedic residents, and a 4.0 -fold difference between the top and bottom $10 \%$ of neurological surgery residents graduating in 2012.

Conclusions: Spine surgery case volumes during orthopaedic and neurological surgery training programs vary greatly both within and between specialties. While orthopaedic residents have shown a steady increase in spine procedures from 2009-2012, they still average less than half of the number of spine procedures compared to neurological surgery trainees. This data may assist with further efforts to optimize contemporary spine surgeon training.

Paper 30. Adjacent Segment Disease In 511 Cases Of Posterolateral Instrumented Lumbar Arthrodesis: Floating Fusion Versus Distal Construct Including The Sacrum

Mohamad Bydon; Risheng Xu, A.M., Ph.D.; David Santiago-Dieppa,
M.D.; Mohamed Macki, B.S.; Rafael De la Garza-Ramos, M.D.; Nicholas B. Abt, B.S.; Robby Weingart; Daniel M. Sciubba, M.D.; Jean-Paul Wolinsky, M.D.; Ali Bydon, M.D.; Ziya L. Gokaslan, M.D.; Timothy F. Witham, M.D.

\section{Johns Hopkins University School of Medicine, Neurosurgery}

Disclosures: M. Bydon: None. R. Xu: None. D. Santiago-Dieppa: None. M. Macki: None. R. De la Garza-Ramos: None. N.B. Abt: None. R. Weingart: None. D.M. Sciubba: 4; I; Medtronic, Nuvasive, Globus, DePuy. 7; I; DePuy. J. Wolinsky: None. A. Bydon: 4; I; MedImmune, LLC. 7; I; DePuy. Z.L. Gokaslan: 6; I; Spinal Kinetics, US Spine. 7; I; DePuy, AOSpine North America, Medtronic, NREF, Integra Life Sciences, K2M. T.F. Witham: 7; I; Eli Lilly and Company.

Introduction: The objective of this study is to examine the longterm outcomes of patients undergoing instrumented posterior fusion of the lumbar spine.

Methods: We present 511 patients who underwent instrumented arthrodesis for lumbar degenerative disease over a 23-year period at a single institution. Patients were followed for an average of $39.73 \pm$ 46.52 months after the index lumbar arthrodesis procedure.

Results: The average age for these patients was 59.4513 .48 years. Of the total 511 patients, $502(98.24 \%)$ presented with back pain, 376 (74.17\%) with radiculopathy, $76(14.87 \%)$ with motor weakness, and $32(6.26 \%)$ with pre-operative bowel/bladder dysfunction. An average of 2.041 .03 spinal levels were fused. Post-operatively, patients experienced a significant improvement in back pain $(\mathrm{p}<0.0001)$ and radiculopathy $(\mathrm{p}<0.0001)$. Patients with fusions excluding the sacrum (floating fusions) were statistically more likely to develop ASD compared with those with fusion constructs ending at S1 distally $(\mathrm{p}=0.030)$, but less likely to develop post-operative radiculopathy ( $\mathrm{p}=0.030)$. In the floating fusion cohort, 31/256 (12.11\%) had cephalad ASD, whereas 39/255 (15.29\%) patients in the lumbo-sacral cohort had cephalad ASD development. This was not statistically different $(p=0.295)$. This suggests that caudad ASD development in the floating fusion cohort is due to the added risk of an unfused L5-S1 vertebral level. Because of the elevated risk of symptomatic radiculopathy but lowered risk of ASD, patients in the lumbosacral fusion cohort had a similar reoperation rate compared to patients undergoing floating fusions ( $\mathrm{p}=0.769)$.

Discussion: Although ASD following spinal operations has been previously described, we present one of the largest cohorts in the Western literature of patients undergoing instrumented fusion for degenerative lumbar spine disease. The incidence of ASD as well as the outcomes of lumbar arthrodesis differs between floating fusions and lumbosacral arthrodesis.

Conclusions: Patients who had floating lumbar fusions were statistically more likely to develop ASD over time compared to those who had lumbosacral fusions incorporating the $\mathrm{S} 1$ spinal segment, but less likely to experience post-operative radicular symptoms. Additional prospective studies may more clearly delineate the long-term risks of instrumented postero-lateral fusions of the lumbar spine.

Paper 31. Risks Factors For Re-operation in Patients Treated Surgically for Degenerative Spondylolisthesis. A subanalysis of the 8 year data from the SPORT trial.

Michael Gerling; Dante Leven; Virginie Lafage; Peter Passius; Nina Bianco; Alex Lee; Jon Lurie; Tor Tosteson; Thomas Errico

SUNY Downstate, orthopedic surgery

Disclosures: M. Gerling: 4; I; Stryker. D. Leven: None. V. Lafage: None. P. Passius: None. N. Bianco: None. A. Lee: None. J. Lurie: 4; I; Fzio Med. 6; I; NewVert. T. Tosteson: None. T. Errico: 1; I; K2M. 


\section{Abstracts of the 2014 Meeting of the Lumbar Spine Research Society}

\begin{tabular}{|c|c|c|c|}
\hline & $\begin{array}{c}\text { Randomized } \\
\text { Cohort } \\
\text { (n=191)t }\end{array}$ & $\begin{array}{c}\text { Observational } \\
\text { Cohort } \\
(n=215) \dagger\end{array}$ & p-value \\
\hline Procedure & & & 0.82 \\
\hline Decompression only & $13(7 \%)$ & $13(6 \%)$ & \\
\hline Non-instrumented fusion & $35(20 \%)$ & $46(22 \%)$ & \\
\hline Instrumented fusion & $128(73 \%)$ & 151 (72\%) & \\
\hline Multi-level fusion & $53(28 \%)$ & $44(20 \%)$ & 0.11 \\
\hline \multicolumn{4}{|l|}{ LaminectomyLevel } \\
\hline L2.L3 & $31(17 \%)$ & $18(9 \%)$ & 0.023 \\
\hline L3.L4 & $107(57 \%)$ & $91(44 \%)$ & 0.009 \\
\hline L4.L5 & $185(97 \%)$ & 204 (96\%) & 0.72 \\
\hline L5.S1 & $67(36 \%)$ & $50(24 \%)$ & 0.011 \\
\hline Levels decompresssed & & & 0.004 \\
\hline 0 & $1(1 \%)$ & $3(1 \%)$ & \\
\hline 1 & $63(33 \%)$ & $104(48 \%)$ & \\
\hline 2 & $71(37 \%)$ & $71(33 \%)$ & \\
\hline $3+$ & $56(29 \%)$ & $37(17 \%)$ & \\
\hline Additional surgeries (1-year rate)\|| & $14(7 \%)$ & $11(5 \%)$ & 0.35 \\
\hline Additional surgeries (2-year rate)\| & 25 (13\%) & $24(11 \%)$ & 0.54 \\
\hline Additional surgeries (3-year rate)|| & 31 (16\%) & 28 (13\%) & 0.36 \\
\hline Additional surgeries (4-year rate)|| & $32(16 \%)$ & $32(15 \%)$ & 0.58 \\
\hline Additional surgeries (5-year rate)\| & 36 (19\%) & 33 (15\%) & 0.35 \\
\hline Additional surgeries (6-year rate)\|" & $39(20 \%)$ & 39 (18\%) & 0.53 \\
\hline Additional surgeries (7-year rate)\| & $41(21 \%)$ & 45 (21\%) & 0.81 \\
\hline Additional surgeries (8-year rate)\| & $44(23 \%)$ & $47(22 \%)$ & 0.70 \\
\hline Recurrent stenosis/progressive spondylolisthesi & $23(13 \%)$ & $18(9 \%)$ & \\
\hline Pseudoarthrosis/fusion exploration & 1 (NE) ${ }^{\prime *}$ & $5(2 \%)$ & \\
\hline Complication or Other & $18(10 \%)$ & $15(7 \%)$ & \\
\hline New condition & $4(2 \%)$ & $9(4 \%)$ & \\
\hline
\end{tabular}

2; I; K2M. 4; I; K2M. 6; I; Fastenetix. 7; I; Paradigm Spine, OMEGA, OREF, Fridolin Trust.

Study Design: A retrospective subgroup analysis of prospectively collected data.

Introduction: Surgery for degenerative spondylolisthesis (DS) is common with good clinical outcomes reported in prospective clinical trials. Though, several high quality studies have examined outcomes following DS surgery, few have identified risk factors for re-operation. Our study hypothesis was that specific patient baseline characteristics would be risk factors for re-operation.

Method: A retrospective subgroup analysis was performed on surgically treated patients enrolled in the DS arm of the multicenter SPORT trial, randomized and observational cohorts. Patients included in the DS arm had neurogenic claudication for at least 12 weeks, clinical neurological signs, spinal stenosis on cross-sectional imaging, and degenerative spondylolisthesis on standing lateral radiographs. In our subgroup analysis, patients were stratified into no re-operation versus re-operation. Baseline characteristics were analyzed using a multivariate regression analysis based on data collected from patients at eight years post-operatively. A Cox regression model Stepwise Method was implemented in SAS with $\mathrm{p}=.0 .10$ significant for entry and $\mathrm{p}=0.05$ significant for retention to the model with calculation of hazard ratios (HR).

Results: Of the 406 surgery patients, $73 \%$ underwent instrumented fusion, $21 \%$ non-instrumented fusion, and $6 \%$ decompression alone. At eight years the re-operation rate was $22 \%, 315$ having no reoperation and 91 in the re-operation group. 25 (28\%) were within the first year, $49(54 \%)$ within 2 years, $64(70 \%)$ within 4 years, and 78 $(86 \%)$ within 6 years. Forty one revisions $(10 \%)$ were for progressive spondylolisthesis, 33 (8\%) for complication or other reason, and 13 (3\%) for a new condition (table 1). Patients with a higher risk for reoperation were more likely to be on antidepressants ( $p=0.008$, HR 2.08) or have pseudoclaudication ( $\mathrm{p}=0.02, \mathrm{HR} 1.82$ ). Patients of older age, smokers, diabetics, obese, or on workman's compensation did not have a greater risk for re-operation (table 2).

Discussion: The incidence of re-operation for patients treated surgically for DS was $22 \%$ at eight years following the initial surgery, with greater than half $(54 \%)$ occurring within the first two years. Patient's with a history of neurogenic claudication and patients taking antidepressants were approximately 1.8 and 2 times as likely to undergo a re-operation, respectively. Additionally, smoking, obesity, diabetes, older age, and workman's compensation were not associated with a higher risk of re-operation.

Paper 32. Risk Factors For Re-operation in Patients Treated Surgically For Lumbar Stenosis. A Subanalysis of the 8 year data from the SPORT trial.

Dante Leven; Peter Passias; Thomas Errico; Virginie Lafage; Nina Bianco; Alex Lee; Jon Lurie; Tor Tosteson; Wenyan Zhao; K. Spratt; Michael Gerling

\section{SUNY Downstate, Orthopedic surgery}

Disclosures: D. Leven: None. P. Passias: None. T. Errico: 1; I; K2M. 2; I; K2M. 4; I; K2M. 6; I; Fastenetix. 7; I; Paradigm Spine, OMEGA, OREF, Fridolin Trust. V. Lafage: None. N. Bianco: None. A. Lee: None. J. Lurie: 4; I; Fzio Med. 6; I; NewVert. T. Tosteson: None. W. Zhao: None. K. Spratt: None. M. Gerling: 4; I; Stryker.

Study design: A retrospective subanalysis of prospectively collected data.

Introduction: Lumbar spinal stenosis ( $\mathrm{SpS})$ involves narrowing of the spinal canal that may lead to pain and disability. SpS is the most common indication for surgery in the elderly population and was the fastest growing spine surgery in the last three decades. Prospective studies have shown that timely surgical intervention can be effective in restoring function, though few long-term studies have identified consistent risk factors for re-operation. Our study hypothesis was that specific patient baseline characteristics would emerge as risk factors for re-operation in patients treated surgically for $\mathrm{SpS}$.

Method: A retrospective subgroup analysis was performed on surgically treated patients enrolled in the SpS arm of the multicenter SPORT trial randomized and observational cohorts. Patients included in the SpS arm had neurogenic claudication for at least 12 weeks and spinal stenosis, without spondylolisthesis or instability, as confirmed on imaging. In our subanalysis, patients were stratified into no reoperation versus re-operation. Baseline characteristics were analyzed

\begin{tabular}{|c|c|c|c|}
\hline & \begin{tabular}{|c|} 
Randomized \\
Cohort \\
$(n=171)^{*}$ \\
\end{tabular} & $\begin{array}{c}\text { Observational } \\
\text { Cohort } \\
(n=246)^{*} \\
\end{array}$ & p-value \\
\hline Procedure & & & 0.53 \\
\hline Decompression only & $143(88 \%)$ & 215 (88\%) & \\
\hline Non-instrumented fusion & $7(4 \%)$ & $15(6 \%)$ & \\
\hline Instrumented fusion & $12(7 \%)$ & $13(5 \%)$ & \\
\hline Multi-level fusion & $5(3 \%)$ & $12(5 \%)$ & 0.46 \\
\hline \multicolumn{4}{|l|}{ LaminectomyLevel } \\
\hline L2-L3 & $58(35 \%)$ & $91(38 \%)$ & 0.62 \\
\hline L3-L4 & $126(75 \%)$ & $160(66 \%)$ & 0.056 \\
\hline L4.L5 & $154(92 \%)$ & 225 (93\%) & 0.92 \\
\hline L5.S1 & $65(39 \%)$ & $92(38 \%)$ & 0.93 \\
\hline Levels decompresssed & & & 0.81 \\
\hline 0 & $4(2 \%)$ & $4(2 \%)$ & \\
\hline 1 & 36 (21\%) & $58(24 \%)$ & \\
\hline 2 & $51(30 \%)$ & $78(32 \%)$ & \\
\hline $3+$ & $80(47 \%)$ & $106(43 \%)$ & \\
\hline Additional surgeries (1-year rate)\| & $7(4 \%)$ & $14(6 \%)$ & 0.46 \\
\hline Additional surgeries (2-year rate) II & $11(6 \%)$ & $21(8 \%)$ & 0.43 \\
\hline Additional surgeries (3-year rate)\| & $16(9 \%)$ & $29(11 \%)$ & 0.43 \\
\hline Additional surgeries (4-year rate)\|I & 22 (13\%) & $32(13 \%)$ & 0.94 \\
\hline Additional surgeries (5-year rate) II & $26(15 \%)$ & 33 (13\%) & 0.66 \\
\hline Additional surgeries (6-year rate)\|I & $28(16 \%)$ & $37(15 \%)$ & 0.75 \\
\hline Additional surgeries (7-year rate)\| & $30(17 \%)$ & $41(16 \%)$ & 0.83 \\
\hline Additional surgeries (8-year rate)\| & $33(19 \%)$ & $44(17 \%)$ & 0.71 \\
\hline Recurrent stenosis/progressive spondylolisthesi & $24(15 \%)$ & $16(7 \%)$ & \\
\hline Pseudoarthrosis/fusion exploration & 0 & 1 (NE)"* & \\
\hline Complication or Other & $6(4 \%)$ & $13(5 \%)$ & \\
\hline New condition & $3(2 \%)$ & $9(4 \%)$ & \\
\hline
\end{tabular}

* For SPS diagnosis, $175 \mathrm{RCT}$ and 253 OBS patients had surgery, surgical information was available for 171 RCT patients and 246 observational patients. Specific procedure available for 171 RCT patients and 246 observational patients.

"One-, two-, three-four-, five-, six-, seven-, and eight-year post-surgical re-operation rates are Kaplan Meier estimates and p-values are based on the log-rank test. Numbers and percentages are based on the first additional surgery if more than one additional surgery. percentages are based on the first additional surgery if more than one additional surgery.
Surgeries include any additional spine surgery not just re-operation at the same level. 


\section{Abstracts of the 2014 Meeting of the Lumbar Spine Research Society}

using a multivariate regression analysis based on data collected from patients at eight years post-operatively. A Cox regression model Stepwise Method was implemented in SAS with $\mathrm{p}=0.10$ significant for entry and $\mathrm{p}=0.05$ significant for retention to the model.

Results: Of the 417 surgery patients, $88 \%$ underwent decompression only, $6 \%$ non-instrumented fusion, and $6 \%$ instrumented fusion. At eight years, the re-operation rate was $18 \%$, with 340 no re-operation and 77 in the re-operation group. 32 (42\%) underwent re-operation within 2 years, $54(70 \%)$ within 4 years, and $65(84 \%)$ within 6 years. Forty $(10 \%)$ patients underwent re-operation for recurrent stenosis, $19(5 \%)$ for a complication or other reason, 12 (3\%) for a new condition. Patients with a lower score on the body pain assessment showed an increased risk for re-operation $(\mathrm{p}=0.049)$. Patients who were smokers, diabetics, obese, on antidepressants, or on workman's compensation did not have a higher risk of re-operation.

Discussion: The rate of re-operation for patients treated surgically for $\mathrm{SpS}$ at eight years post-operatively was $18 \%$ with $70 \%$ occurring within the first four years. Patients with a lower body pain score had an increased risk for re-operation. Other baseline characteristics were not associated with an increased risk for re-operation. This study highlights the low re-operation rates following lumbar laminectomy in spinal stenosis patients without deformity or spondylolisthesis.

Paper 33. Secreted Phosphoprotein 24 Kd (Spp24) Inhibits Nerve

Root Inflammatio

Haijun Tian; Chenshuang Li; Trevor P. Scott; Scott R. Montgomerey; Kevin Phan; Lifeng Lao; Wei Zhang; Yawei Li; Tetsuo Hayashi; Shinji Takahashi; Raed Alobaidaan; Monchai Ruangchainikom; Ke-Wei Zhao; Elsa J. Brochmann; Samuel S. Murray; Jeffrey C. Wang; Michael D. Daubs

\section{Shanghai Changzheng Hospital, Orthopedic Surgery Department}

Disclosures: H. Tian: None. C. Li: None. T.P. Scott: None. S.R. Montgomerey: None. K. Phan: None. L. Lao: None. W. Zhang: None. Y. Li: None. T. Hayashi: None. S. Takahashi: None. R. Alobaidaan: None. M. Ruangchainikom: None. K. Zhao: None. E.J. Brochmann: None. S.S. Murray: None. J.C. Wang: 1; I; Biomet, Stryker, Medtronics, Depuy-Synthes, Amedica, Osprey, Aesculap, Seaspine. 6; I; Fziomed, Promethean spine, Paradigm spine, Benevenue, NexGen, Pioneer, Amedica, Vertiflex, Electrocore, Surgitech, Axiomed, VG Innovations, Corespine, Expanding orthopaedics, Syndicom, Osprey, Bone biologics, Curative biosciences. M.D. Daubs: 1; I; Dupuy-Synthes. 4; I; Dupuy-Synthes. 7; I; Stryker.

Introduction: Recently the potential for an inflammatory effect of BMP-2 on exposed nerve roots has been raised. No reliable solution for this obstacle to the application of BMP-2 in this setting has been promulgated. We have undertaken studies of the use of a BMP-2 binding protein, secreted phosphoproteins $24 \mathrm{kD}$ (Spp24), as a potential therapeutic molecule to dampen the inflammatory effects of BMP-2.

Methods: Dorsal root ganglion cells were cultured with BMP-2 and markers of neuro-inflammation were measured by immunohistochemistry. Rats were randomized into four different groups according to the implantation they received: collagen sponge only, BMP-2 only, BMP-2 + collagen sponge, and BMP-2 + Spp24 and received a left unilateral L5 laminotomy and protein implantation. Functional evaluation was done using the Basso-Beattie-Bresnahan (BBB) scale. Routine H\&E staining and immunohistochemical analyses using CGRP and Substance P staining were done.

Results: Treatment of DRG cells in vitro with BMP-2 significantly increased CGRP expression whereas treatment with Spp24 did not. The CGRP inductive effect of BMP-2 was blocked by the addition of Spp24 into the culture medium (Fig 1). Two days after surgery the BBB score for the subjects from the BMP-2 and BMP-2+Sponge groups decreased to a value that was significantly less than that of the control group. The neurologic function of the BMP-2+Spp24 group was not different from that of the control group and was higher than both the BMP-2 and BMP-2+Sponge groups. H\&E staining revealed that the myelin sheath was swollen in the treatment BMP-2 group. Immunohistochemical analyses demonstrated that the BMP-2 only and the BMP-2+ Sponge groups had increased expression of both CGRP and Substance P, while the addition of the Spp24 effectively blocked this effect.

Discussion: By binding to BMP-2, Spp24 can reduce BMP-2 induced neuro-inflammation both in vitro and in animal subjects treated with BMP-2 in vivo. A well designed Spp24 sponge can both work as a binding barrier as well as a physical barrier to BMP-2 protein.

Conclusion: Spp24 is an ideal candidate for preventing BMP-2 induced neuro-inflammation.

Paper 34. Secondary Hypersensitivity to Subthreshold Stimuli Persists following Resolution of Acute Disc Herniation Neuropathy and is Mediated by Heightened TRPV1 Receptor Function

Mohammed F. Shamji, MD, PhD; Yu-Shan Tu, MSc; Michael W. Salter, MD, PhD

\section{Toronto Western Hospital, Neurosurgery}

Disclosures: M.F. Shamji: None. Y. Tu: None. M.W. Salter: None.

Introduction: While acute disc-herniation induced radiculopathy most frequently resolves without clinical sequelae, a fraction of patients experience long-term sensory dysfunction. This study examined chronic sensitivity of the rodent hindpaw following resolution of acute inflammatory neuropathy.

Methods: C57BL/6 mice underwent mid-thigh sciatic nerve dissection, with either exposure only (control) or placement of nucleus pulposus (NP). Animals were evaluated throughout one, three, and five weeks for mechanical allodynia, thermal hyperalgesia, cold allodynia, and gait stability. At each time point, animals received intraplantar injection of capsaicin $(0.1 \mu \mathrm{g})$ or vehicle alone, thereafter the same behavioral testing. Immunohistochemistry was performed of sciatic nerve, dorsal root ganglion (DRG), and spinal cord for inflammatory activation as well as TRPV1 receptor expression. Ex vivo DRG explants were assessed for capsaicin sensitivity to TRPV1 activation (cobalt staining).

Results: Upon resolution of acute inflammatory pain, mice at three and five weeks but not one week demonstrated profound mechanical allodynia to subthreshold capsaicin compared with sham-operated controls or NP-stimulation animals delivered vehicle only (Figure

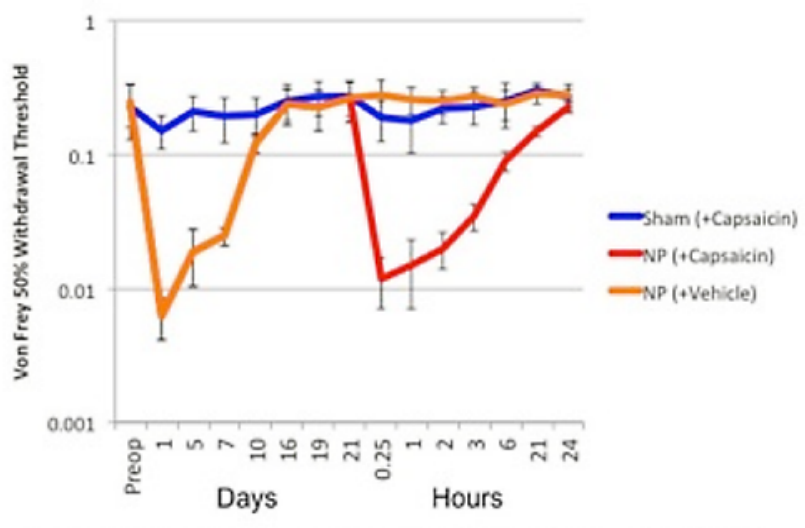

Figure I: Radiculopathy animak appear seasitized to a secondary stimulus. Sham animals (blue) exposed to suhthreshold intraplantar capsaicin exhibit no response. Upon resolution of mechanical seasitivity. NP animals exposed to subthreshold capsaicin (red) eshibit 2 profound response that was not experienced by those treated with vehicle aloae (orange). 


\section{Abstracts of the 2014 Meeting of the Lumbar Spine Research Society}

1 for 3 weeks, $\mathrm{p}<0.01)$. Conversely, perineural lymphocyte and intraneural macrophage infiltration was only observed at one week. Heightened spinal cord dorsal horn and DRG TRPV1 expression were seen, and DRG explants derived from NP-treated animals exhibited greater cobalt staining upon capsaicin stimulation compared with controls.

Conclusions: Non-compressive disc herniation sensitizes the sciatic nerve distribution in this animal model, despite resolution of acute intraneural macrophage migration. The demonstrated role of TRPV1 may explain how acute inflammatory pain transforms into chronic neuropathic pain. Decreasing TRPV1 expression may prevent the development of the long-term painful phenotype.

Paper 35. Expression Of Degenerative, Inflammatory And Neurotrophic Markers In Lumbar Discs During Normal Aging

Josemberg Silva Baptista, PhD; Ricardo B. Fontes, MD, PhD; Vincent C. Traynelis, MD; Edson A. Liberti, PhD

\section{Rush University Medical Center, Neurosurgery}

Disclosures: J.S. Baptista: None. R.B. Fontes: None. V.C. Traynelis: 1; I; Medtronic. 4; I; Medtronic. 6; I; Medtronic. 7; I; Globus. E.A. Liberti: None.

Introduction: Intervertebral discs (IVDs) undergo significant structural modifications during normal aging, a process mediated by inflammatory cytokines while the effector enzymes are those of the MMP family and their antagonists, TIMPs. Neurotrophins promote proliferation of nerve fibers thought to be relevant in the pathogenesis of discogenic pain. These mediators have been reported in discs from symptomatic individuals and generally thought to be pathologic. Our objective was to analyze the expression of TNF, IL-1, VEGF, NGF, BDNF, TIMP-1 and MMPs-1, -2 and -3 in presumably asymptomatic young (Group 1; $<35$ years) and elderly (Group 2; >65 years) individuals.

Methods: Each group included 30 lumbar IVDs (L4-5 and L5-S1) from 15 individuals; a thorough family interview excluded symptomatic individuals. Mediator expression was analyzed with immunohis-

\section{Expression of studied molecules}

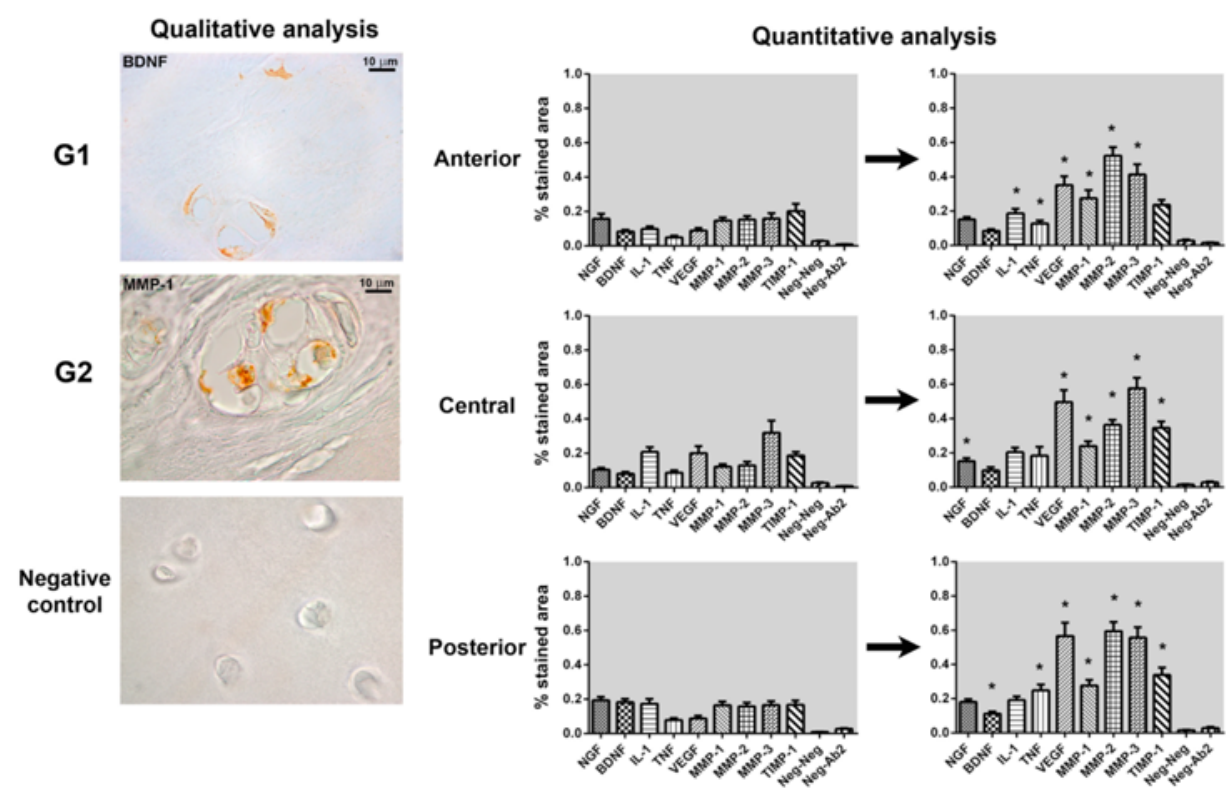

tochemistry in different disc sectors, quantified with an area-based assay and compared between groups (young vs. elderly).

Results: Studied molecules were detected in every disc sector of both G1 and G2. Most statistical comparisons (18/27, 66.7\%) revealed an increase in mediator expression in $\mathrm{G} 2$ in relation to G1. A senescent profile with elevated VEGF, MMP-1, $-2,-3$ and TIMP-1 was found in every disc sector. Elevated TNF levels were characteristic of the AF and elevated NGF, in the NP. The only mediator to be decreased in the elderly was BDNF in the posterior AF.

Conclusions: The mere presence of any of the studied molecules cannot be considered pathologic. Expression of remodeling enzymes and inflammatory mediators is similar across lumbar disc regions, leading to a common degenerated pattern with elevated VEGF, MMP-1, $-2,-3$ and TIMP-1 while TNF was more elevated in the AF. Despite structural findings of degeneration, elevated mediator expression suggest this remodeling process is ongoing even at 65 years of age and older. Molecular studies with discs from asymptomatic individuals are important to understand the process of normal aging and identify targets for modulation.

Paper 36. Extracellular Matrix Remodeling In Lumbar Discs During Normal Aging

Ricardo Braganca Fontes, MD, PhD; Josemberg Silva Baptista, PhD; Said R. Rabbani, PhD; Vincent C. Traynelis, MD; Edson A. Liberti, $\mathrm{PhD}$

\section{Rush University Medical Center, Neurosurgery}

Disclosures: R.B. Fontes: None. J.S. Baptista: None. S.R. Rabbani: None. V.C. Traynelis: 1; I; Medtronic. 4; I; Medtronic. 8; I; Globus. E.A. Liberti: None.

Introduction: A complete description of the degenerative modifications of the extracellular matrix (ECM) of human intervertebral discs (IVDs) from a well-controlled population is lacking. Utilization of surrogates for the normal human disc (discs from other animal species, adjacent discs obtained during surgery in symptomatic individuals, etc), age heterogeneity and undisclosed disc region are some of the main problems. Collagens are the most important macromolecules of the ECM but alterations in their profile have never been quantified during normal aging. Our objective was to describe and compare the structure, ultrastructure and collagen profile of lumbar IVDs of young(Group1; \&lt35years) and elderly (Group2; \&gt65 years) presumably asymptomatic individuals

Methods: Each age group included 30 lumbar IVDs (L4-5 and L5-S1) collected from 15 individuals; a thorough family interview excluded symptomatic individuals. IVDs were analyzed with MRI, a macroscopic grading scale, histology, scanning electron microscopy (SEM) and immunohistochemistry for collagen subtypes I, II, III, IV, V, VI, IX and X. Collagen expression was quantified and compared between G1 and G2.

Results: Degenerative features were found in both groups but were significantly worse in $\mathrm{G} 2$ as shown by MRI and macroscopic grading scales, as expected. Aging was associated 
A
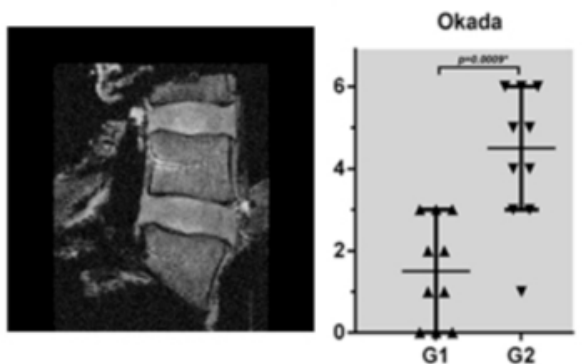

B
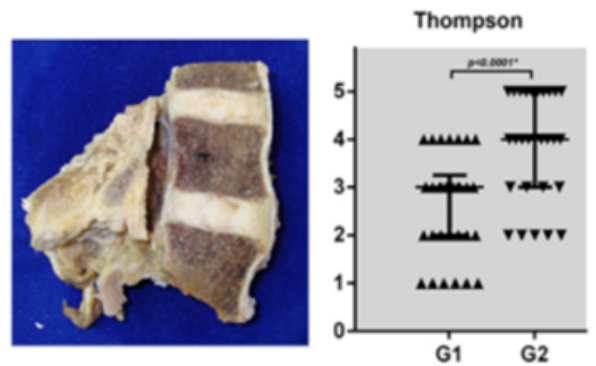

C

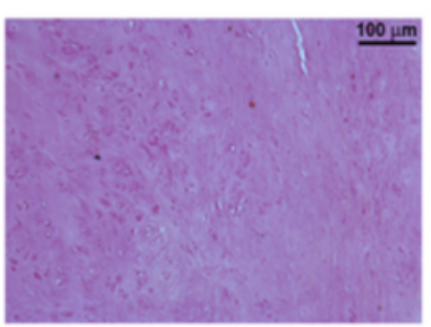

D

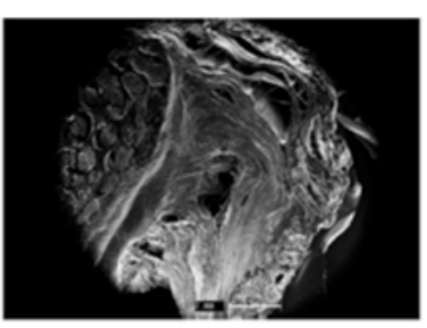

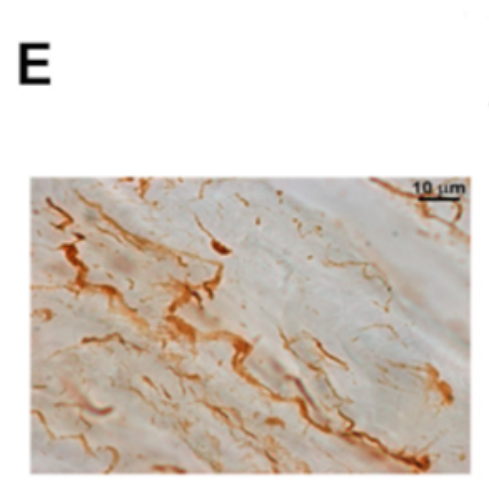

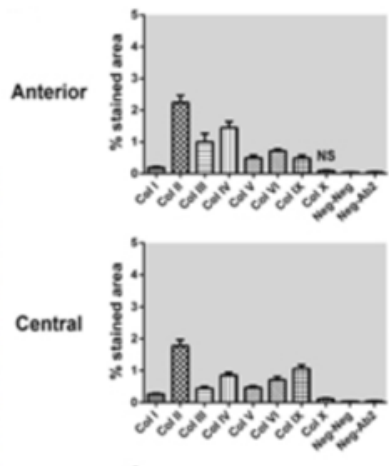

Posterior

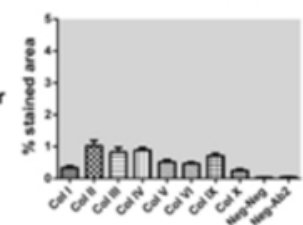

with an increase in density of annular fibers, endplate hypertrophy and chondrocyte proliferation. SEM demonstrated that basic disc features not seen in older specimens by $1.5 \mathrm{~T}$ MRI were still present, such as Sharpey-type endplate insertion of annular fibers and a distinct nucleus even in the most degenerated specimens. Collagen II was the most important collagen subtype in the lumbar IVD with important components of collagen IV (annulus) and IX (nucleus). Collagen expression is reduced differently in the elderly annulus (collagens II, III, IV) and nucleus (collagens V, VI, IX). Collagen X was only detected in young IVDs.

Conclusions: Normal aging of the IVD is a complex remodeling process with loss of fibrous features and acquisition of a cartilaginous phenotype. Collagen expression is significantly reduced during aging; this occurs differently according to disc region. A morphological roadmap for aging of the ECM is thus proposed which may be useful to plan restorative strategies.

Figure 1A-B. A: \% DHI 0 over time (days, dotted line indicates $\mathrm{DHI}_{0}$ ), $B$ : Polarized light histology with micro-CT reconstructions of motion segments A.

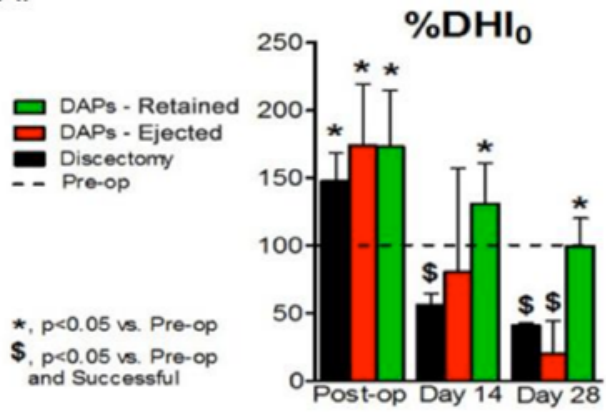

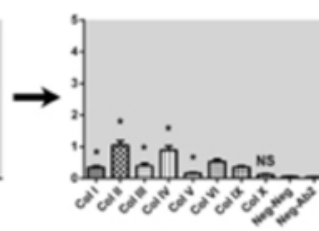
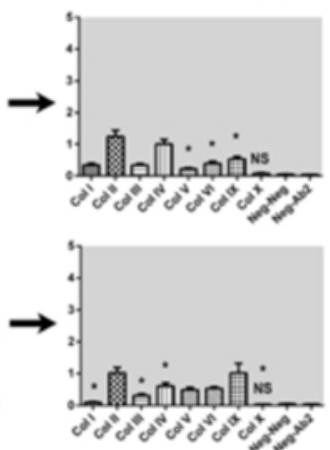

Paper 37. Nanofibrous Disc-like Angle Ply Structures for Intervertebral Disc Tissue Engineering in a Small Animal Model

Andrew H. Milby, MD; John T. Martin, MS; Lachlan J. Smith, PhD; Joseph A. Chiaro, BS; Harvey E. Smith, MD; Dawn M. Elliott, PhD; Robert L. Mauck, PhD

University of Pennsylvania, Orthopaedic Surgery

Disclosures: A.H. Milby: None. J.T. Martin: None. L.J. Smith: None. J.A. Chiaro: None. H.E. Smith: None. D.M. Elliott: None. R.L. Mauck: None.

Introduction: Tissue engineering may offer solutions for disc degeneration that avoid the limitations of fusion or disc replacement. A nanofibrous annulus fibrosus (AF) scaffold patterned after native tissue and seeded with AF cells has produced disc-like angle ply structures (DAPS) in vitro. We investigated the ability of acellular DAPS constructs to maintain disc height in vivo in the rat caudal disc space.

Methods: Electrospun $\operatorname{poly}(\varepsilon-$ caprolactone) (PCL) was collected on a rotating mandrel as aligned fibrous sheets. Two strips with alternating \pm 30 degree alignment were then wrapped concentrically to mimic the AF. Mature Sprague-Dawley rats were divided into 4 groups: 1) 14-day discectomy-only $(n=4), 2)$ 14-day discectomy with DAPS $(n=6), 3)$ 28-day discectomy-only $(n=4)$, 4) 28-day discectomy with DAPS $(n=11)$. Postoperatively, Disc Height Index (DHI) was monitored via fluoroscopy, and Micro-CT assessed vertebral alignment and remodeling. Histology sections were stained with $\mathrm{H} \& \mathrm{E}$ and viewed under brightfield or polarized light.

Results: For the discectomy-only group, a transient expansion was followed by gradual collapse to $40 \%$ of pre-op DHI by day 28 . DAPS were retained in the disc space in $47 \%$ ( 8 of 17) of cases ("retained" group), with DHI for the retained group showing no significant change at 28 days. The remaining 9 animals demonstrated ejection of the DAPS from the disc space
B.

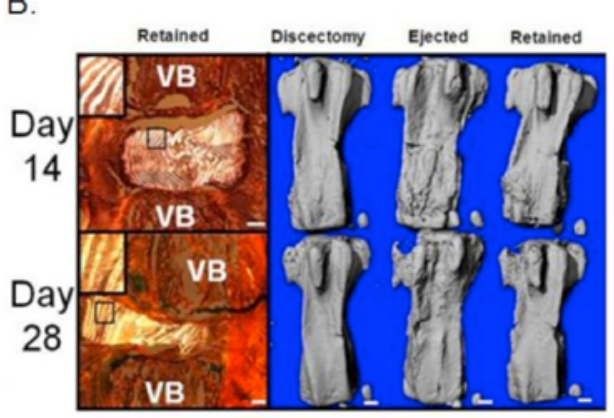




\section{Abstracts of the 2014 Meeting of the Lumbar Spine Research Society}

("ejected" group), with loss of DHI similar to that of the discectomyonly group. Micro-CT confirmed maintenance of disc height in the retained group, with minor misalignment and bony remodeling at 28 days. In the ejected group, there was remodeling of the vertebrae that was not observed in the discectomy-only group.

Discussion: When retained in the disc space, acellular AF-only DAPS maintained disc height in the rat caudal spine over 28 days. The unpredictable loading environment of the rat tail and minimal cellular infiltration likely contributed to inconsistent retention of the DAPS.

Conclusions: Tissue-engineered AF scaffolds have the potential to maintain disc height in vivo in the rat caudal disc model. Future directions include temporary external fixation to promote early tissue integration, as well as sacrificial fibers to increase porosity and enhance cellular invasion.

Paper 38. Contrast-Enhanced Micro-Computed Tomography $(\mu \mathrm{CT})$ Is Sensitive To In Vitro And In Vivo Degenerative Changes Of The Intervertebral Disc

Kraig Kristof, MD; Michael D. Newton, BS; Tristan Maerz, MS; Olesya Motovylyak, BS; Jeffrey S. Fischgrund, MD; Daniel K. Park, $\mathrm{MD} ;$ Kevin C. Baker, $\mathrm{PhD}$

\section{Beaumont Health System, Orthopaedic Research}

Disclosures: K. Kristof: 2; I; Ohio Rheumatologic Association. M.D. Newton: None. T. Maerz: None. O. Motovylyak: None. J.S. Fischgrund: 2; I; Multiple sources. 4; I; Stryker, Relievant. 6; I; Baxano Surgical. 8; I; Multiple sources (manuscript preparation), Baxano Surgical (board membership), Multiple sources (expert testimony). D.K. Park: 4; I; Stryker, K2M, DePuy. 6; I; Johnson \& Johnson. K.C. Baker: None.

Objective: Assessment of small animal models of degenerative disc disease (DDD) using conventional imaging techniques is challenging. The objective of this study was to employ equilibrium partitioning of an ionic contrast agent (EPIC)- $\mu$ CT to characterize degenerative changes in both in vitro and in vivo models of DDD.

Materials and Methods: In phase I, excised lumbar intervertebral discs (IVDs) from 4 healthy New Zealand White rabbits were digested in vitro via intradiscal papain injection for $0,24,48$, and 72 hours.

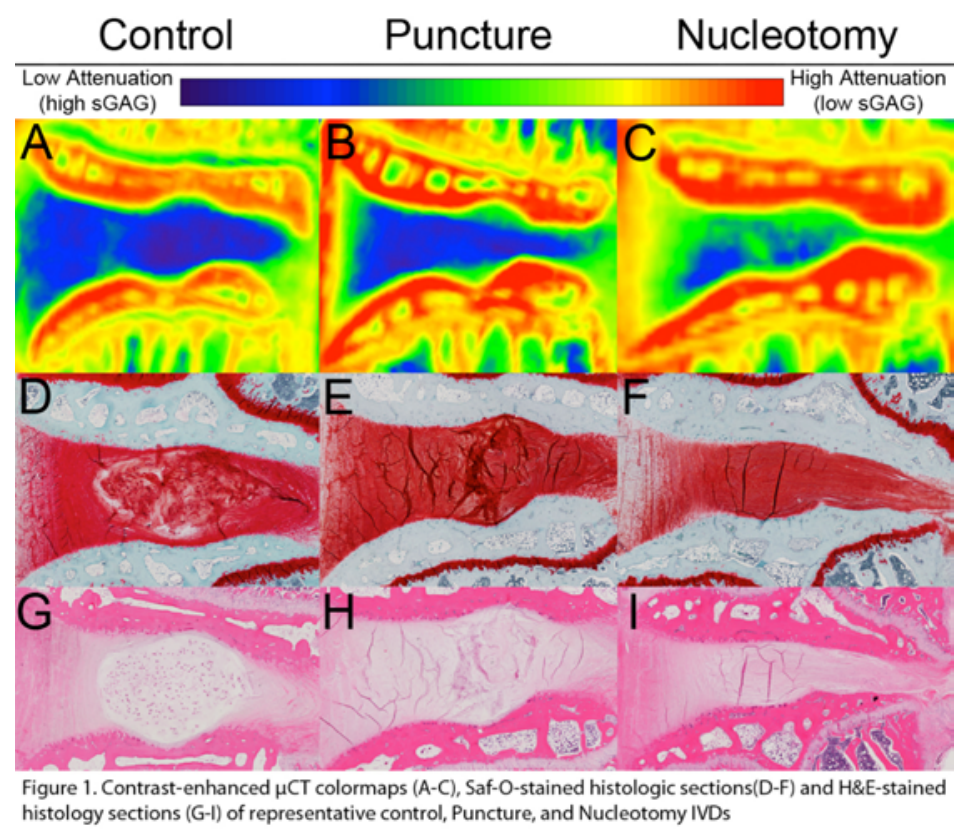

In phase II, degeneration was induced surgically in 12 New Zealand White rabbits via annular puncture and nucleotomy and harvested after 3 and 6-weeks postoperatively. IVD degeneration was assessed using EPIC- $\mu \mathrm{CT}$, qualitative histologic grading, and disc height measured from sagittal histologic sections.

Results: Phase I results demonstrate that $\mathrm{EPIC} \mu \mathrm{CT}$ is sensitive to molecular changes in the IVD. Papain digestion increased attenuation of the NP by $23.4 \% \pm 40$ at $24 \mathrm{hrs}(\mathrm{P}=.18)$, significantly by $42.8 \% \pm 34$ at $48 \mathrm{hrs}(\mathrm{P}=.033)$, and by $37.8 \% \pm 25$ at $72 \mathrm{hrs}(\mathrm{P}=.09)$. NP attenuation significantly correlated to qualitative histologic score $(\mathrm{r}=0.502$, $\mathrm{P}=.024)$. Results of Phase II demonstrate that EPIC- $\mu$ CT effectively characterizes in vivo IVD degeneration (Figure 1). Attenuation of the NP increased by $17.8 \% \pm 24$ in punctured discs and significantly by $54.1 \% \pm 24$ in Nucleotomy IVDs $(\mathrm{P}=.014)$ at 3 -weeks, and increased by $9.18 \% \pm 24$ in Puncture IVDs and significantly by $29.1 \% \pm 12$ $(\mathrm{P}<.001)$ in Nucleotomy IVDs at 6-weeks. There were significant correlations between histologic grade and NP attenuation (Nucleotomy: $\mathrm{r}=0.708, \mathrm{P}<.001$; Puncture: $\mathrm{r}=0.490, \mathrm{P}=.015)$ and between disc height and NP attenuation (Nucleotomy: $\mathrm{r}=-.708, \mathrm{P}<.001$; Puncture: $\mathrm{r}=-.723$, $\mathrm{P}<.001)$.

Discussion: Significant increases in NP attenuation (compared to controls) at 48 hours in Phase I and in Nucleotomy IVDs in Phase II demonstrate that EPIC- $\mu \mathrm{CT}$ can effectively differentiate between healthy and severely degraded IVDs. Significant correlations to disc height and qualitative histology score, both established measurements of DDD, demonstrate that EPIC- $\mu \mathrm{CT}$ sensitively reflects the level of degeneration for both in vitro and in vivo models of DDD.

Conclusion: EPIC $-\mu \mathrm{CT}$ is sensitive to both in vitro and in vivo rabbit models of DDD and provides a high resolution method for mapping degenerative changes in rabbit IVDs.

Paper 39. The Reliability and Validity of the Thoracolumbar Injury Classification System in Pediatric Spine Trauma

Jason W. Savage, MD; Timothy Moore, MD; Paul Arnold, MD; Wellington K. Hsu, MD; Alpesh Patel, MD; Kathryn McCarthy, MD; Gregory D. Schroeder, MD; Alexander Vaccaro, MD, PhD.; John Dimar, MD; Paul A. Anderson, MD

\section{University of Wisconsin, Orthopedics and Rehabilitation}

Disclosures: J.W. Savage: 4; I; Stryker. T. Moore: None. P. Arnold: 4; I; Medtronic, Lifespine, Integra Life, Spine Wave, Stryker Spine. 6; I; Z-plasty. W.K. Hsu: 4; I; AONA, Lifenet, Medtronic, Pioneer Surgical, Stryker, Terumo, Zimmer. 7; I; Baxter. A. Patel: 1; I; Amedica, Ulrich Medical USA. 4; I; Amedica, Biomet, Depuy, GE Healthcare, Stryker, Zimmer. 6; I; Amedica, Cytonics, Nocimed, Trinity Orthopedics. 9; I; Springer Publishing. K. McCarthy: None. G.D. Schroeder: None. A. Vaccaro: 1; I; Aesculap, Biomet Spine, DePuy, Globus, Medtronic, Nuvasive, Stryker Spine. 4; I; Gerson Lehrman Group, Guidepoint Global,Innovative Surgical Design, Medacrop, Orthobullets, Stout Medical. 6; I; Advanced spinal intellectual properties, Bonovo orthopaedics, Computational biodynamics, Cross current, Cytonics, Electrocore, Flagship surgical, Flowpharma, Gamma spine, In vivo, Innovative surgical, K2 medical, Locationa based intelligence, paradigm spine, progressive spinal technologies, RSI, Replication medica, Rothman Institute and Related Properties, Small bone innovations, Spine medica, Spinicity, Spinology, Stout medical, Syndicom. J. Dimar: None. P.A. Anderson: 1; I; Stryker, Pioneer Surgical. 4; I; Pioneer, Aesculap, Medtronic. 6; I; Pioneer, Titan Surgical, Expanding Orthopedics, SI bone.

Introduction: The thoracolumbar injury classification system (TLICS) was developed to improve the categorization and management of thoracolumbar trauma. TLICS has been shown to 


\begin{tabular}{|l|l|}
\hline Category & kappa \\
\hline Injury Morphology & 0.619 \\
\hline Neurological Status & 0.958 \\
\hline PLC & 0.524 \\
\hline Treatment & 0.449 \\
\hline
\end{tabular}

have good reliability and validity in the adult population. The purpose of this study was to determine the clinical applicability of the TLICS in pediatric spine trauma.

Methods: The clinical and radiographic findings of 20 pediatric thoracolumbar fractures were prospectively presented to 20 surgeons with varying levels of training and experience with spinal trauma. These injuries were consecutively scored using the TLICS. Cohen's unweighted kappa coefficients and Spearman's rank order correlation values were calculated for the key parameters (injury morphology, status of PLC, neurological status, and proposed management) to assess the inter-rater reliabilities. 5 surgeons scored the same cases 2 months later to assess the intra-rater reliability. The actual management of each case was then compared with the treatment recommended by the TLICS algorithm to assess validity.

Results: The inter-rater kappa statistics of all subgroups (injury morphology, status of the posterior ligamentous complex, neurological status, and proposed treatment ) were within the range of moderate to substantial reproducibility (0.449-0.958) (Table 1). Proposed treatment had the lowest inter-rater agreement (0.449). All subgroups had excellent intra-rater reliability $(0.748-1.000)$. The various indices for validity were calculated ( 80.3 percent correct, 0.836 sensitivity, 0.785 specificity, 0.676 positive predictive value, 0.899 negative predictive value).

Conclusions: The thoracolumbar injury classification system has good reliability and validity when used in the pediatric population. The interrater reliability of predicting management and indices for validity are lower than in adults with thoracolumbar fractures, which is likely due to differences in the way children are treated for certain types of injuries. TLICS can be used to reliably categorize thoracolumbar injuries in the pediatric population; however, modifications may be needed to better guide treatment in this specific patient population.
Paper 40. The Role of Athletic Activity on Lumbar Structural Abnormalities in Adolescent Patients with Symptomatic Low Back Pain

Gregory D. Schroeder, M.D.; Marco C. Mendoza, M.D.; Erika L. Daley; Cynthia R. LaBella, M.D.; Jason W. Savage, M.D.; Alpesh A. Patel, M.D. FACS; Wellington K. Hsu, M.D.

Northwestern University Feinberg School of Medicine, Orthopaedic Surgery

Disclosures: G.D. Schroeder: None. M.C. Mendoza: None. E.L. Daley: None. C.R. LaBella: 9; I; american academy of pediatrics. J.W. Savage: None. A.A. Patel: 1; I; Amedica, Ulrich Medical USA. 4; I; Amedica, Biomet, DePuy, GE Healthcare, Stryker, Zimmer. 6; I; Amedica, Cytonics, Nocimed, Trinity Orthopaedics. 9; I; Springer. W.K. Hsu: 4; I; AONA, Lifenet, Medtronic, Pioneer Surgical, Stryker, Terumo, Zimmer. 7; I; Baxter.

Introduction: It is unknown whether elite athletes are predisposed to a higher prevalence of lumbar spine pathology. Although some authors have opined that these changes may occur during skeletal immaturity, it is unclear if adolescent athletes are at an increased risk.

Methods: Records of pediatric patients (ages 10-18) between 20042012 were evaluated. Patients with symptomatic low back pain, a lumbar spine MRI, and reported athletic activity met the inclusion criteria. Patients who participated in sports at least 5 days or 20 hours per week, or were on a varsity team were stratified to the "athlete" group. A control "non-athlete" group included patients who participated in sports $<3$ days or $<5$ hours per week. Athletes were also stratified into contact, hyperextension, and rotational activities. Lumbar MRIs were blinded and reviewed by two authors. Each intervertebral disc was assigned a Pfirrmann grade and evaluated for a herniated disc and/or pars fracture. The senior author graded pathology in cases of disagreement.

Results: 66 athletes and 48 non-athletes were identified. BMI, smoking history and pelvic incidence (48.5 versus 48.2 respectively, $\mathrm{p}=0.94$ ) were similar between the groups. The overall prevalence of lumbar pathology was 55\%, and athletes were more likely to have

\begin{tabular}{|c|c|c|c|c|c|c|c|}
\hline \multicolumn{8}{|c|}{ Table 1: } \\
\hline & $\begin{array}{c}\text { All Non- } \\
\text { Athletes } \\
(48)\end{array}$ & $\begin{array}{c}\text { Contact } \\
\text { Athletes } \\
(29)^{*}\end{array}$ & P Value & $\begin{array}{l}\text { Hyper- } \\
\text { Extension } \\
\text { Athletes } \\
(21)^{* *}\end{array}$ & $\mathrm{P}$ Value & $\begin{array}{c}\text { Rotation } \\
\text { Athletes } \\
(18)^{* * *}\end{array}$ & P Value \\
\hline $\begin{array}{c}\text { Pfirrmann } \\
\text { average }\end{array}$ & 1.14 & 1.28 & 0.17 & 1.09 & 0.26 & 1.16 & 0.87 \\
\hline $\begin{array}{l}\% \text { with at } \\
\text { least one } \\
\text { degenerative } \\
\text { disc }\end{array}$ & $31 \%$ & $45 \%$ & 0.33 & $33 \%$ & $<1.00$ & $28 \%$ & $<1.00$ \\
\hline $\begin{array}{c}\text { \% with a } \\
\text { HNP }\end{array}$ & $33 \%$ & $34 \%$ & $<1.00$ & $33 \%$ & $<1.00$ & $11 \%$ & 0.12 \\
\hline $\begin{array}{c}\% \text { with a } \\
\text { pars fracture }\end{array}$ & $2 \%$ & $17 \%$ & 0.03 & $38 \%$ & 0.0002 & $56 \%$ & 0.00002 \\
\hline $\begin{array}{l}\% \text { with a at } \\
\text { least one } \\
\text { degenerative } \\
\text { disc, a HNP } \\
\text { and/or a } \\
\text { pars fracture }\end{array}$ & $40 \%$ & $66 \%$ & 0.04 & $67 \%$ & 0.07 & $72 \%$ & 0.03 \\
\hline \multicolumn{8}{|c|}{$\mathrm{P}$ value compares the group of athletes and the non athlete control group } \\
\hline \multirow{2}{*}{\multicolumn{8}{|c|}{$\begin{array}{c}\text { - Contact sports were defined by the American Academy of Pediatrics. Athletes in the group played } \\
\text { Basketball (14), Soccer (11), Football (4), Lacrosse (2) and Wrestling (1) } \\
\text {-*Hyperextension sports included: Gymnastics (12); Cheerleading (2); Ballet(2); Dance (2); Pole } \\
\text { Vault (1); and Ice Skating (1) }\end{array}$}} \\
\hline & & & & & & & \\
\hline ***Rotation & Athletes in & lude: Bas & all/Softbal & 10); Tennis ( & ; Lacrosse & 2); and $\mathrm{Bac}$ & inton (1) \\
\hline
\end{tabular}




\section{Abstracts of the 2014 Meeting of the Lumbar Spine Research Society}

lumbar pathology $(67 \%$ versus $40 \%, \mathrm{p}=0.01)$. Specifically, the risk of a pars injury was increased in athletes (32\% vs. $2 \%, \mathrm{p}=0.0003)$; however, there was no difference in the average Pfirrmann grade (1.19 versus $1.14, \mathrm{p}=0.41)$, percentage of patients with at least one degenerative disc (26 athletes (39\%) versus 15 non-athletes $(31 \%)$ ), or rate of disc herniation (18 athletes $(27 \%)$ versus 16 non-athletes $(33 \%))$. Contact, hyperextension, and rotation athletes demonstrated an increased risk of a pars fracture but no difference in incidence of degenerative or herniated discs (Table 1).

Conclusion: Adolescents with significant low back pain have a higher-than-expected prevalence of structural pathology regardless of athletic activity. Although competitive athletes demonstrated a higher rate of pars fracture than non-athletes, there was no difference in the risk of degenerative changes or disk herniation.

Paper 41. Percutaneous Lumbopelvic Instrumentation for Highly Unstable Sacral Fractures with Spino-Pelvic Dissociation

Seth K. Williams, MD; Stephen M. Quinnan, MD

University of Wisconsin School of Medicine, Department of Orthopedics and Rehabilitation

Disclosures: S.K. Williams: 4; I; DePuy Synthes Spine. S.M. Quinnan: 2; I; Smith \& Nephew, Orthofix. 4; I; Smith \& Nephew, Orthofix.

Introduction: Sacral fractures with spinopelvic dissociation patterns are highly unstable injuries that often require surgical treatment. In an effort to minimize the morbidity of surgery while achieving stable fixation, we developed a percutaneous lumbopelvic fixation technique. The purpose of this study was to determine the complications and outcomes of percutaneous lumbopelvic fixation in the treatment of highly unstable sacral fractures with spinopelvic dissociation

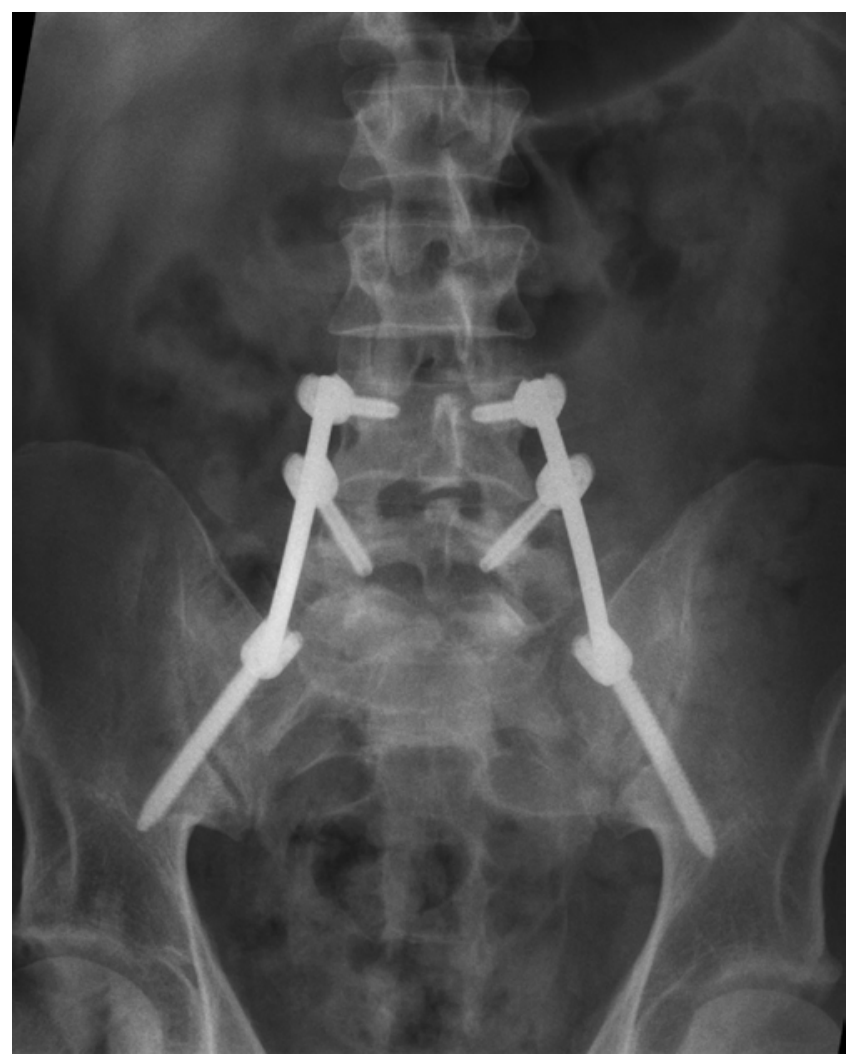

patterns. This is a prospective consecutive case series.

Methods: From March 2008 through July 2013, 20 consecutive patients with unstable sacral fractures were treated with percutaneous lumbopelvic fixation. Data were collected prospectively including blood loss, fluoroscopy time, screw accuracy, wound complications, neurological status, cases of instrumentation failure, fracture healing, and patient functional status. Clinical results were assessed with selfreport measures including the SF-12, ODI, and WHO-5. Radiological results included comparison of pre- and post-operative CT scans and $\mathrm{x}$-rays in all patients.

Results: Surgical duration averaged 2 hours 18 minutes and blood loss averaged $180 \mathrm{~mL}$. There was 1 deep wound infection and 1 wound dehiscence. Post-operative CT scans showed a $2 \mathrm{~mm}$ medial pedicle breach, a $3 \mathrm{~mm}$ lateral pedicle breach, and a $2 \mathrm{~mm}$ iliac screw cortical breech (all asymptomatic) but otherwise accurate intraosseous pedicle screw $(n=90)$ and iliac screw placement $(n=42)$. All but one patient maintained or regained full neurological function, and all patients healed their fractures. Clinical follow-up in 15 patients who had surgery more than one year prior to this report averaged 918 days. Radiographic follow-up averaged 737 days, with all fractures healed. There were no instrumentation failures, although one patient had pedicle screw loosening at 2 years post-operatively. Ten patients underwent elective instrumentation removal.

Conclusions: Sacral fractures with spinopelvic dissociation patterns are severe injuries. This is the first reported series of patients treated with a percutaneous lumbopelvic instrumentation technique. Percutaneous lumbopelvic instrumentation can be performed safely with limited blood loss and within a reasonable surgical duration, theoretically minimizing the physiological burden of surgery in these severely injured patients.

Paper 42. 243 Surgical Discectomy Patients From The SPORT Trial Compared To 8,350 Discectomy Patients From ACS NSQIP: Demographics, Perioperative Variables, And Complications Correlate Well

Nicholas S. Golinvaux, BA; Daniel D. Bohl, MPH; Bryce A. Basques, BS; Jonathan N. Grauer, MD

\section{Yale University School of Medicine, Department of Orthopaedics}

Disclosures: N.S. Golinvaux: None. D.D. Bohl: None. B.A. Basques: None. J.N. Grauer: 2; I; Stryker. 4; I; Affinergy, Alphatec, Bioventus, Depuy, Harvard Clinical Reserach Institute, Powered Research, Stryker, Transgenomic.

Introduction: The Spine Patient Outcomes Research Trial (SPORT) was a prospective randomized clinical trial that randomized patients with lumbar pathology to receive treatment of either surgery or continued conservative measures. Although there were study limitations, such as study group crossover, this highly quoted work has become a benchmark to which other studies are compared. The growing utilization of national databases for analyzing large patient cohorts now offers a way to assess the greater generalizability of the SPORT discectomy data.

Methods: Based on current procedural terminology (CPT) and International Classification of Diseases, 9th revision (ICD-9) coding, we identified all elective lumbar discectomies logged in the American College of Surgeons National Surgical Quality Improvement Program (ACS NSQIP) database between 2010 and 2012. This database includes hospital and 30-day follow-up data. Where possible, based on published available data, demographics, perioperative factors, and complications were compared between the SPORT and NSQIP discectomy patients.

Results: A total of 8,350 discectomy patients were identified in NSQIP, and these were compared to the 243 discectomy patients from SPORT. Demographics between the two groups were similar: 
Table 1: Comparison of demographics and complications between SPORT and NSQIP

\begin{tabular}{|c|c|c|c|}
\hline \multirow[t]{2}{*}{ Demographics } & $\begin{array}{r}\text { SPORT - Randomized to } \\
\text { surgery }\end{array}$ & $\begin{array}{l}\text { SPORT - Randomized to } \\
\text { non-operative treatment }\end{array}$ & NSQIP \\
\hline & $\mathrm{n}(\%)$ & $\mathrm{n}(\%)$ & $\mathrm{n}(\%)$ \\
\hline Overall & $232(49)$ & $240(51)$ & $8,350(100)$ \\
\hline Age, mean (SD) & $41.7 \mathrm{y}(11.8)$ & $43 \mathrm{y}(11.3)$ & $48.6 \mathrm{y}(14.7)$ \\
\hline \multicolumn{4}{|l|}{ Sex } \\
\hline Male & $131(56)$ & $147(61)$ & $4,648(56)$ \\
\hline Female & $101(44)$ & $93(39)$ & $3,702(44)$ \\
\hline Current smoker & $47(20)$ & $61(25)$ & $2,290(27)$ \\
\hline \multicolumn{4}{|l|}{ Race/ethnicity } \\
\hline Non-Hispanic & $223(96)$ & $225(94)$ & $7,835(94)$ \\
\hline White & $197(85)$ & $202(84)$ & $6,899(83)$ \\
\hline BMI, mean (SD) & $27.8(5.6)$ & $28.2(5.4)$ & $29.7(6.4)$ \\
\hline Complications & SPORT - Underwent surgery $\$$ & & NSQIP \\
\hline Overall & $243(100)$ & & $8,350(100)$ \\
\hline Operation time, mean (SD), min & $79.1(36.3)$ & & 88.5 (SD 51.9) \\
\hline \multicolumn{4}{|l|}{ Length of Stay } \\
\hline Same day & $65(27)$ & & $3,038(36.4)$ \\
\hline 1 Night & $137(57)$ & & $3,615(43.3)$ \\
\hline$>1$ Night & 37 (15) & & $1,697(20.3)$ \\
\hline \multicolumn{4}{|l|}{ Intraoperative complications } \\
\hline Dural tear/spinal fluid leak & $10(4)$ & & N/A NSQIP \\
\hline Vascular injury & $1(0)$ & & N/A NSQIP \\
\hline Other & $2(1)$ & & N/A NSQIP \\
\hline None & $230(95)$ & & N/A NSQIP \\
\hline Wound complications* & $4(2)$ & & $86(1.0)$ \\
\hline Postoperative blood transfusion & $0(0)$ & & $79(0.9)$ \\
\hline Blood loss & $64.7(88.4)$ & & N/A NSQIP \\
\hline
\end{tabular}

average age was within 7 years, gender distribution within $1 \%$, smoking status within $7 \%$, ethnicity distribution within $2 \%$, and BMI within 0.8. Perioperative factors and complications between the two groups were also similar: average operative time within $10 \mathrm{~min}$, wound complications within $1 \%$, and postoperative blood transfusion within $1 \%$. Length of stay was also similar between the SPORT and NSQIP cohorts with $84 \%$ vs $80 \%$ going home within a day of surgery, respectively.

Discussion: Although a highly regarded study, the discectomy arm of the SPORT trial had limited patient numbers and some crossover between treatment groups. That said, available data elements were remarkably comparable to those found in a much larger cohort from the ACS NSQIP database.

Conclusion: Where comparisons were possible, based on available data, the demographics, perioperative factors, and complication rates from the SPORT discectomy trial do appear to be a reasonable approximation of those that would be found in a large, national patient database. These findings support the greater generalizability of the SPORT discectomy trial results.

Paper 43. Pre-existing Lumbar Spine Diagnoses as a Predictor of Outcomes in National Football League Athletes

Gregory D. Schroeder; Thomas Sean Lynch, M.D.; Daniel Gibbs, M.D.; Ian Chow; Mark Labelle; Alpesh A. Patel, M.D. FACS; Jason W. Savage, M.D.; Wellington K. Hsu, M.D.; Gordon W. Nuber, M.D.

Northwestern University Feinberg School of Medicine, Orthopaedic Surgery

Disclosures: G.D. Schroeder: None. T.S. Lynch: None. D. Gibbs: None. I. Chow: None. M. Labelle: None. A.A. Patel: 1; I; Amedica, Ulrich Medical USA. 4; I; Amedica, Biomet, DePuy, GE Healthcare, Stryker, Zimmer. 6; I; Amedica, Cytonics, Nocimed, Trinity Orthopaedics. 9; I; Springer. J.W. Savage: None. W.K. Hsu: 4; I; AONA, Lifenet, Medtronic, Pioneer Surgical, Stryker, Terumo, Zimmer. 7; I; Baxter. G.W. Nuber: None.
Introduction: It is currently unknown how pre-existing lumbar spine conditions may affect the medical evaluation, draft status, and subsequent career performance of National Football League (NFL) players. Outcomes of athletes with preexisting lumbar spine pathology may help aid players' and practitioners' expectations of career longevity and success.

Methods: The written medical evaluations and imaging reports of prospective NFL players from 2003-2011 from one franchise during the combine were evaluated. Players with a reported lumbar spine diagnosis and with appropriate imaging were included in this study. Athletes were then matched by age, position, year, and round drafted to control draftees without a lumbar spine diagnosis. Career statistics and performance scores were calculated.

Results: Out of a total of 2,965 athletes evaluated, 414 were identified as having a pre-existing lumbar spine diagnosis (figure 1). Players without a lumbar spine diagnosis were more likely to be drafted than those with one $(80.2 \%$ vs. $61.1 \%$ respectively, $\mathrm{p}<0.001)$. Drafted athletes with a pre-existing lumbar spine injury compared to the matched control group had no difference in the number of years played ( 4.0 vs. 4.3 years, respectively, $\mathrm{p}=0.13$ ), games played ( 46.5 vs. 50.7 , respectively, $\mathrm{p}=0.15$ ), games started ( $28.1 \mathrm{vs.}$ 30.6 , respectively, $\mathrm{p}=0.39$ ) or performance score (1.4 vs. 1.8 , respectively, $\mathrm{p}=0.3$ ). Compared to controls, the diagnoses of spondylosis $(62.37 \%$ vs. $78.55 \%, \mathrm{P}<0.001)$, a lumbar herniated disc $(60.27 \%$ vs. $78.43 \%, \mathrm{P}<0.001)$, or spondylolysis $+/$ - a slip $(64.44 \%$ vs. $78.15 \%, \mathrm{P}$ $<0.001)$ led to a decrease in the likelihood of an athlete being drafted, but there was no affect on career longevity or performance. Notably, two athletes who had undergone a posterior lateral lumbar fusion were drafted. One played in 125 games, and the other is still active and has played in 108 games.

Conclusion: This study suggests that a pre-existing lumbar spine diagnosis was associated with a lower draft status for NFL athletes, however, such a diagnosis did not affect a player's career longevity or performance. We also report players with a lumbar fusion who have achieved a successful career in the NFL.

\begin{tabular}{|c|c|}
\hline \multicolumn{2}{|c|}{$\begin{array}{l}\text { Figure 1: Diagnosis for players with a history of a } \\
\text { lumbar spine injury }\end{array}$} \\
\hline Degenerative spondylosis* & 186 \\
\hline Herniated disc* & 146 \\
\hline $\begin{array}{l}\text { Spondylolysis with or } \\
\text { without spondylolisthesis* }\end{array}$ & 135 \\
\hline Strain & 90 \\
\hline Stenosis* & 19 \\
\hline Fracture* & 17 \\
\hline SI joint pain & 11 \\
\hline Scoliosis* & 6 \\
\hline Scheurmann's kyphosis* & 3 \\
\hline ^ Based on imaging reports & \\
\hline
\end{tabular}


Abstracts of the 2014 Meeting of the Lumbar Spine Research Society

\begin{tabular}{|c|c|c|c|}
\hline & Open & Percutaneous/MIS & $\begin{array}{l}\mathrm{p} \text { - } \\
\text { value }\end{array}$ \\
\hline$n=256$ & 157 & 99 & \\
\hline Median Age (IQR) & $42(25-54)$ & $48(27-65)$ & 0.09 \\
\hline Female & 43 & 33 & 0.31 \\
\hline Mean GCS (SD) & $13.7(3)$ & $13.6(2.8)$ & 0.8 \\
\hline Mean ISS (SD) & $26.5(1.1)$ & $25.5(1.4)$ & 0.57 \\
\hline Length of stay (IQR) & $6.8(4.9-14)$ & $7.6(4.7-19.9)$ & 0.41 \\
\hline Mean Vent Days (SD) & $9.7(12.7)$ & $12.1(13.3)$ & 0.88 \\
\hline Mean mobilize day (SD) & $3.6(4.9)$ & $4(6.4)$ & 0.57 \\
\hline Median OR time (IQR) & $129.5(104.5-200)$ & $94(60-133)$ & 0.0001 \\
\hline Median EBL (IQR) & $450(300-700)$ & $75(50-150)$ & 0.0001 \\
\hline Transfusion & 81 & 24 & 0.0001 \\
\hline I\&D for infection & 8 & 5 & 1 \\
\hline Revision & 0 & 3 & 0.001 \\
\hline Pneumonia & 10 & 6 & 0.92 \\
\hline Mortality -in hospital & 4 & 2 & 0.69 \\
\hline $\begin{array}{l}\text { Complication (PNA, } \\
\text { Mortality, Infection, Revision) }\end{array}$ & 21 & 19 & 0.693 \\
\hline
\end{tabular}

Paper 45. Risk Factors For Re-operation Following Discectomy and Laminectomy in Patients With Intervertebral Disc Herniations. A subanalysis of the 8 year data from the SPORT trial.

Dante Leven; Peter Passias; Thomas Errico; Virginie Lafage; Nina Bianco; Alex Lee; Jon Lurie; Tor Tosteson; Michael Gerling

\section{SUNY Downstate, Orthopedic surgery}

Disclosures: D. Leven: None. P. Passias: None. T. Errico: 1; I; K2M. 2; I; K2M. 4; I; K2M. 6; I; Fastenetix. 7; I; Paradigm Spine, OMEGA, OREF, Fridolin Trust. V. Lafage: None. N. Bianco: None. A. Lee: None. J. Lurie: 4; I; Fzio Med. 6; I; NewVert. T. Tosteson: None. M. Gerling: 4; I; Stryker.

Study Design: A retrospective subgroup analysis of prospectively collected data

Introduction: Lumbar discectomy and laminectomy for patients with intervertebral disc herniations (IDH) is the most common spine surgery performed in the United

Paper 44. Comparison of Open versus Percutaneous Posterior Spine Fixation in The Treatment of Thoracolumbar fractures: a retrospective chort study.

Abimbola Obafemi-Afolabi

University of Maryland, Orthopaedics

Disclosures: Obafemi-Afolabi: None.

Introduction: Percutaneous minimally invasive posterior spinal stabilization is sometimes the only option in the treatment of critically ill poly-trauma patients who require spinal stabilization but are physiologically incompetent to undergo traditional open procedures. The objective is to show that when compared to traditional open posterior spine surgery, percutaneous minimally invasive approach has better or equivalent outcomes.

Methods: A retrospective cohort chart review. After IRB obtained, trauma database was reviewed for all patients with thoracolumbar fractures treated with posterior spine instrumentation. Exclusion criteria were fractures due to infections \& tumors and fractures requiring an anterior approach.

Results: 425 posterior spine instrumentation cases identified from January 2007-May 2013. 256 patients met inclusion criteria. 157 cases done via traditional open approach while 99 were done percutaneously. A statistical significant difference, favoring percutaneous fixation, was seen in median operative time, blood loss and transfusions. There were 3 instrumentation revisions in the percutaneous fixation group and none in the traditional open approach group.

Conclusions: With less operative time, blood loss and transfusion requirements, percutaneous posterior spine fixation is a valid treatment option for thoracolumbar fractures; however, there is a higher rate of revision surgery with this approach compared to the traditional open posterior spine approach.
States with variable reported re-operation rates. Though prospective studies have reported outcomes related to this patient population, few have examined risk factors for re-operation. Our study hypothesis was that specific patient baseline characteristics would emerge as risk factors for re-operation in patients treated surgically for IDH's.

Method: A retrospective subgroup analysis was performed on surgically treated patients enrolled in the IDH arm of the multicenter SPORT trial, randomized and observational cohorts. Patients included in the IDH arm had radicular pain for at least six weeks, clinical evidence of nerve-root irritation, and imaging showing a disc herniation at a level and side consistent with the patient's symptoms. In our subgroup analysis patients were stratified into no re-operation versus re-operation. Baseline characteristics were analyzed using a multivariate regression analysis based on data collected from patients at eight years post-operatively. A Cox regression model Stepwise Method was implemented in SAS with $\mathrm{p}=0.10$ significant for entry and $\mathrm{p}=0.05$ significant for retention to the model with calculation of hazard ratios (HR).

Results: At eight years, the re-operation rate was $14.6 \%$ with 691 having no re-operation and 119 in the re-operation group. 48 (40\%) of patients underwent re-operation within the first year, $66(55 \%)$ at two years, $85(71 \%)$ at four years, $102(86 \%)$ at six years. $74(10 \%)$

\begin{tabular}{|c|c|c|c|}
\hline & $\begin{array}{c}\text { Randomized } \\
\text { Cohort } \\
(n=262)\end{array}$ & $\begin{array}{c}\text { Observational } \\
\text { Cohort } \\
(n=548)\end{array}$ & p-value \\
\hline \multicolumn{4}{|l|}{ Discectomy Level } \\
\hline L2-L3 & $3(1 \%)$ & $12(2 \%)$ & 0.47 \\
\hline L3-L4 & $8(3 \%)$ & $20(4 \%)$ & 0.85 \\
\hline L4-L5 & $102(40 \%)$ & $217(40 \%)$ & 0.94 \\
\hline L5-S1 & $152(59 \%)$ & $306(56 \%)$ & 0.43 \\
\hline Additional surgeries (1-year rate) \|| & $11(4 \%)$ & $37(7 \%)$ & 0.13 \\
\hline Additional surgeries (2-year rate) \| & $16(6 \%)$ & $50(9 \%)$ & 0.12 \\
\hline Additional surgeries (3-year rate) \|| & $20(7 \%)$ & $53(10 \%)$ & 0.29 \\
\hline Additional surgeries (4-year rate) \|| & $24(9 \%)$ & $61(11 \%)$ & 0.32 \\
\hline Additional surgeries (5-year rate) \| & $25(9 \%)$ & $65(12 \%)$ & 0.27 \\
\hline Additional surgeries (6-year rate) \| & $29(11 \%)$ & $73(13 \%)$ & 0.31 \\
\hline Additional surgeries (7-year rate) \| & $33(12 \%)$ & $79(14 \%)$ & 0.40 \\
\hline Additional surgeries (8-year rate) ॥| & $35(13 \%)$ & $84(15 \%)$ & 0.38 \\
\hline Recurrent disc herniation & $17(7 \%)$ & $57(11 \%)$ & \\
\hline Complication or Other & $9(3 \%)$ & $21(4 \%)$ & \\
\hline New condition & $3(1 \%)$ & $10(2 \%)$ & \\
\hline
\end{tabular}

II One-, two-, three- four-, five-, six-, seven-, and eight-year post-surgical reoperation rates are Kaplan Meier estimates and p-values are based on the log. rank test. Numbers and percentages are based on the first additional surgery if more than one additional surgery. Surgeries include any additional spine surgery not just re-operation at the same level. 


\section{Abstracts of the 2014 Meeting of the Lumbar Spine Research Society}

patients underwent re-operation for a recurrent disc herniation, 30 (4\%) for complication or other factor, and $13(2 \%)$ reported as a new condition (table 1). Patients with a history of antidepressant use had an increased risk for re-operation $(\mathrm{p}=0.0366)$. Patients who were smokers, diabetics, obese, workman's compensation, or clinically depressed did not have a greater risk for re-operation (table 2).

Discussion: In patients undergoing discectomy and laminectomy for IDH, the overall re-operation rate was $14.6 \%$ at eight years postoperatively with greater than half $(55 \%)$ occurring within the first two years. Patient history of smoking, obesity, diabetes, depression and workman's compensation were not associated with a higher risk for re-operation. A history of antidepressant use did correlate with an increased risk for re-operation, and should be considered when counseling patients for surgery.

Paper 46. The Impact of Diabetes Mellitus in Surgical Outcomes after Lumbar Spine Surgery

Alejandro Marquez-Lara, MD; Sreeharsha V. Nandyala, BA; Steven J. Fineberg, MD; Kern Singh, MD

\section{Rush University Medical Center, Orthopaedic Surgery}

Disclosures: A. Marquez-Lara: None. S.V. Nandyala: None. S.J. Fineberg: None. K. Singh: 1; I; Pioneer, Stryker, Zimmer. 4; I; Zimmer, Stryker, Globus. 8; I; Vital 5 LLC. 9; I; Thieme, LWW.

Introduction: Diabetes Mellitus (DM) is a prevalent comorbidity among patients undergoing spine surgery. The purpose of this study was to utilize a population-based database to determine the impact of $\mathrm{DM}$ on the outcomes, costs, and mortality after lumbar spine surgery (LSS).

Methods: The Nationwide Inpatient Sample (NIS) database was queried from 2002-2011. Patients undergoing an elective lumbar decompression (LD) or a lumbar fusion (LF) were identified. The selected cohorts were further divided into three groups: 1) Non diabetics, 2) Diabetics without end-organ damage (Uncomplicated), and 3) Diabetics with end-organ damage (Complicated). Patient demo-

\begin{tabular}{|c|c|c|c|}
\hline \multicolumn{4}{|c|}{ Patients with Uncomplicated Diabetes Mellitus } \\
\hline Variable & Odds Ratio & $\begin{array}{l}\text { 95\% Confidence } \\
\text { Interval }\end{array}$ & P-value \\
\hline Pulmonary Embolism & 0.89 & $0.66-2.26$ & 0.44 \\
\hline Deep Vein Thrombosis & 1.0 & $0.61-1.66$ & 0.64 \\
\hline Infection & 1.1 & $0.93-1.34$ & 0.23 \\
\hline Cardiac Event & 1.2 & $0.97-1.59$ & 0.31 \\
\hline Hematoma & 0.98 & $0.76-1.3$ & 0.89 \\
\hline Hemorrhagic Anemia & 1.1 & $1.04-1.13$ & $<0.001$ \\
\hline Neurologic Complication & 0.76 & $0.64-0.90$ & 0.002 \\
\hline Urinary Tract Infection & 1.12 & $1-.03-1.22$ & 0.006 \\
\hline Ileus & 0.89 & $0.82-0.96$ & 0.003 \\
\hline Gastrointestinal Hemorrhage & 0.58 & $0.37-0.92$ & 0.02 \\
\hline Mortality & 1.2 & $0.79-1.80$ & 0.39 \\
\hline \multicolumn{4}{|c|}{ Patients with Diabetes Mellitus w/ End-Organ Damage (Complicated) } \\
\hline Variable & Odds Ratio & $\begin{array}{l}\mathbf{9 5} \% \text { Confidence } \\
\text { Interval }\end{array}$ & P-value \\
\hline Pulmonary Embolism & 0.33 & $0.12-0.90$ & 0.03 \\
\hline Deep Vein Thrombosis & 1.18 & $0.61-2.27$ & 0.62 \\
\hline Infection & 2.21 & $1.58-3.09$ & $<0.001$ \\
\hline Cardiac Event & 0.91 & $0.65-1.26$ & 0.55 \\
\hline Hematoma & 0.88 & $0.61-1.28$ & 0.88 \\
\hline Hemorrhagic Anemia & 1.3 & $1.18-1.49$ & $<0.001$ \\
\hline Neurologic Complication & 0.75 & $0.46-1.20$ & 0.23 \\
\hline Urinary Tract Infection & 1.49 & $1.23-1.80$ & $<0.001$ \\
\hline Ileus & 1.03 & $0.83-1.28$ & 0.79 \\
\hline Gastrointestinal Hemorrhage & 0.95 & $0.37-2.43$ & 0.91 \\
\hline Mortality & 2.0 & $0.93-4.3$ & 0.07 \\
\hline \multicolumn{4}{|c|}{$\begin{array}{l}\text { *0dds ratios reported from multivariate regression controlling for demographics, } \\
\text { comorbidities, and hospital characteristics } \\
\text { ** Reference is "no diabetes" }\end{array}$} \\
\hline
\end{tabular}

graphics, comorbidities (CCI), length of stay (LOS) and costs were compared between these three groups. Multinomial logistic regression identified the risk factors associated with postoperative complications in diabetics after controlling for age, other comorbidities, and hospital variables. SPSS v.20 was utilized for statistical analysis and a p-value $<0.001$ denoted statistical significance.

Results: A total of 292,833 LDs and 263,263 LFs were identified in the NIS database. Diabetic patients represented $15.1 \%(n=44,357)$ and $14.5 \%(n=38,067)$ of the LD and LF cohorts respectively. In both cohorts, diabetic patients were significantly older and demonstrated a greater comorbidity burden. Patients with DM incurred a significantly greater LOS, total hospital costs and postoperative complications than non-diabetics. In addition, diabetics demonstrated a greater mortality rate than non-diabetics in both the LF and LD cohorts. Regression analysis demonstrated that uncomplicated DM was a predictor of postoperative neurologic complications, urinary tract infections (UTI), ileus, hemorrhagic anemia, and gastrointestinal (GI) bleeds. Similarly, complicated DM was a predictor of pulmonary emboli (PE), infections, UTI, and hemorrhagic anemia.

Discussion/Conclusions: Diabetic patients who underwent a lumbar spinal procedure incurred a greater LOS, total hospital costs, postoperative complications and mortality than non-diabetics. In addition, diabetes was strongly associated with postoperative infections, hemorrhagic anemia, PE, and UTI. Further research is warranted to demonstrate if preoperative blood glucose control will help mitigate the risk of postoperative complications and mortality that is associated with this prevalent comorbidity.

Paper 47. Complications, Length Of Stay, and Readmission Following Iliac Crest Bone Graft Use in Spinal Fusion: A Multivariate Analysis of Patients in the ACS NSQIP Database

Jordan A. Gruskay, BA; Bryce A. Basques, BS; Matthew L. Webb, AB; Jonathan N. Grauer

Yale University School of Medicine - Dept of Orthopedics and Rehabilitation

Disclosures: J.A. Gruskay: None. B.A. Basques: None. M.L. Webb: None. J.N. Grauer: 2; I; Stryker. 4; I; Affinergy, Alphatec, Bioventus, Depuy, Harvard Clinical Research Institute, Powered Research, Stryker, Transgenomic.

Introduction: The use of iliac crest bone graft (ICBG) in spinal fusion has been associated with increased surgical time, increased hospital length of stay (LOS), and donor site morbidity associated with the harvest procedure. Development of expensive bone graft substitutes has been predicated on these issues. The current study uses a large, national database to compare outcomes for those receiving ICBG to those who did not using multivariate analysis to control for confounding factors.

Methods: A retrospective review of prospectively collected data from the American College of Surgeons National Surgical Quality Improvement Project (ACS NSQIP) 2010-2012 database was conducted. The database was queried for patients undergoing spinal fusion with or without ICBG using CPT codes. Bivariate and multivariate analyses were performed to determine the effect of harvesting ICBG on operative time, postoperative adverse events, LOS, and readmission while controlling for comorbidities, demographics, and approach.

Results: 15,198 patients undergoing spinal fusion were identified. Of these, 698 (4.6\%) utilized ICBG. Bivariate logistic regression (used for categorical variables) found the ICBG cohort was more likely to have a postoperative blood transfusion $(13.0 \%$ vs. $6.2 \%, \mathrm{p}<0.001$ ). Bivariate linear regression (used for continuous variables) found the ICBG cohort to have an extended operative time $(+39.9 \mathrm{~min}, \mathrm{p}<0.001)$ and extended LOS $(+0.7$ days, $\mathrm{p}<0.001)$ relative to those for whom ICBG was not utilized. Multivariate analyses controlling for comorbidities, demograph- 


\section{Abstracts of the 2014 Meeting of the Lumbar Spine Research Society}

ics, and approach determined postoperative blood transfusion (OR $2.2)$ and extended operative time $(+38.5 \mathrm{~min}, \mathrm{p}<0.001)$ to be significantly associated with ICBG use.

No other adverse event was significantly associated with ICBG use. Readmission rates were not significantly different.

Discussion: The current study used a large national database cohort (698 of whom received ICBG) and identified extended operative time and postoperative blood transfusion to be the only short-term outcomes associated with ICBG on multivariate analysis. Meanwhile, the extended LOS seen in ICBG patients is likely driven by comorbidities and anatomic approach.

Conclusion: ICBG was confirmed to be associated with extended operative time and blood transfusion. Serious adverse events, infection, extended LOS, and increased readmission rates are not independently associated with ICBG use.

\section{Poster Presentation Abstracts}

Poster 1. Lumbar Disc Herniation as a Predictor of PerformanceBased Outcomes in National Football League Athletes

Gregory D. Schroeder; Thomas Sean Lynch, M.D.; Daniel Gibbs, M.D.; Mark Labelle; Ian Chow; Alpesh A. Patel, M.D. FACS; Jason W. Savage, M.D.; Wellington K. Hsu, M.D.; Gordon W. Nuber, M.D.

Northwestern University Feinberg School of Medicine, Orthopaedic Surgery

Disclosures: G.D. Schroeder: None. T.S. Lynch: None. D. Gibbs: None. M. Labelle: None. I. Chow: None. A.A. Patel: 1; I; Amedica, Ulrich Medical USA. 4; I; Amedica, Biomet, DePuy, GE Healthcare, Stryker, Zimmer. 6; I; Amedica, Cytonics, Nocimed, Trinity
Orthopaedics. 9; I; Springer. J.W. Savage: None. W.K. Hsu: 4; I; AONA, Lifenet, Medtronic, Pioneer Surgical, Stryker, Terumo, Zimmer. 7; I; Baxter. G.W. Nuber: None.

Introduction: Literature suggests that athletes who develop a lumbar disc herniation while playing in the NFL can return to have a successful career. However, the effects of a pre-existing herniated lumbar disc on the career longevity and performance of NFL athletes is unknown.

Methods: The written medical evaluations and imaging reports of prospective NFL athletes from 2003-2011 during the combine were evaluated. Players with the diagnosis of a herniated lumbar disc were included in this study. Drafted athletes were matched by age, position, year- and round-drafted to control draftees without a lumbar spine diagnosis. Drafted players with a herniated lumbar disc were compared to controls and stratified based on type of treatment and level of herniation.

Results: A total of 2,965 athletes were evaluated, and 146 were identified with a previous lumbar disc herniation. Players without a history of a lumbar herniated disc were more likely to be drafted than those with one $(78.4 \%$ vs. $60.3 \%$ respectively, $\mathrm{p}<0.001)$. Eightyeight athletes with a previous herniated lumbar disc were drafted, and compared to matched controls, they had no difference $(p>0.05)$ in the number of years played, games played, games started, or performance score. However, $5 \%$ (4 athletes) of players with a prior lumbar disc herniation required surgery in the NFL, compared to $0 \%$ of matched controls $(\mathrm{p}=0.04)$ When affected athletes were stratified by either previous treatment or herniation level and compared to controls, neither affected games played, games started, or performance score $(p>$ $0.05)$ (Table 1). When athletes who underwent surgical and conservative treatment were compared to each other, there was no difference ( $p>0.05)$ in number of years played (4.2 versus 3.9), games played (49.0 versus 45.5 ), games started ( 31.7 versus 24.5 ), or performance score (2.4 versus 1.6).

Conclusion: This study suggests that a pre-existing lumbar herniated disc was associated with a lower draft status for NFL athletes, and an increased risk of lumbar spine surgery in the NFL; however, the diagnosis did not affect players' career longevity or performance. These results are independent of operative versus non-operative treatment and level of herniation.

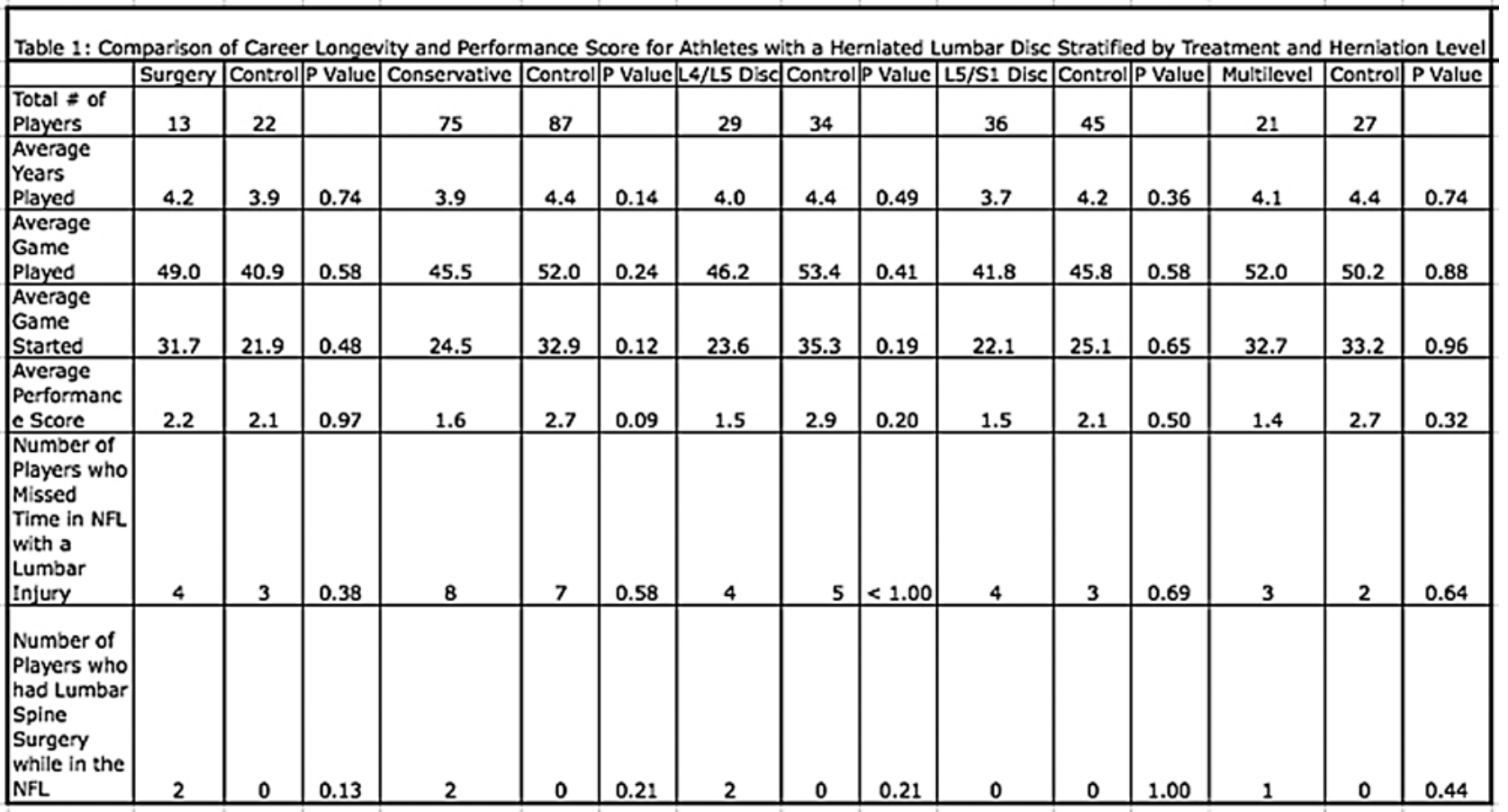




\section{Abstracts of the 2014 Meeting of the Lumbar Spine Research Society}

Poster 2. Results of Database Studies Vary Depending on the Database Used: A Study of the Inter-database Reliability of NIS and NSQIP for Lumbar Fusion Procedures

Daniel D. Bohl, M.P.H.; Glenn S. Russo, M.D.; Bryce A. Basques, B.S.; Nicholas S. Golinvaux, B.A.; Michael C. Fu, B.S.; William D. Long III, M.D.; Jonathan N. Grauer, M.D.

\section{Yale University}

Disclosures: D.D. Bohl: None. G.S. Russo: None. B.A. Basques: None. N.S. Golinvaux: None. M.C. Fu: None. W.D. Long III: None. J.N. Grauer: 2; I; Stryker. 4; I; Affinergy, Alphatec, Bioventus, Depuy, Harvard Clinical Research Institute, Powered Research, Stryker, Transgenomic.

Introduction: The field of orthopaedic surgery has seen a marked increase in the use of national databases to conduct research. Questions regarding the validity and consistency of such studies have not been well explored.

Methods: A retrospective cohort study of patients undergoing lumbar fusion procedures during 2009-2011 was performed in two national databases: the Nationwide Inpatient Sample (NIS) and the National Surgical Quality Improvement Program (NSQIP). Demographics, hospital lengths of stay, comorbidities, and inpatient adverse events were directly compared between databases.

Results: 144,098 and 8,434 patients undergoing lumbar fusion procedures were identified from NIS and NSQIP, respectively. Demographics and hospital lengths of stay were similar between the two databases.

There were substantial differences between the two databases in the prevalences of three comorbidities: The prevalences of obesity and anemia in NSQIP were more than twice those in NIS, and the prevalence of peripheral vascular disease in NIS was more than twice that in NSQIP. Five other comorbidities had prevalences that were within a two-fold difference between the two databases.

Similarly, there were substantial differences between the two

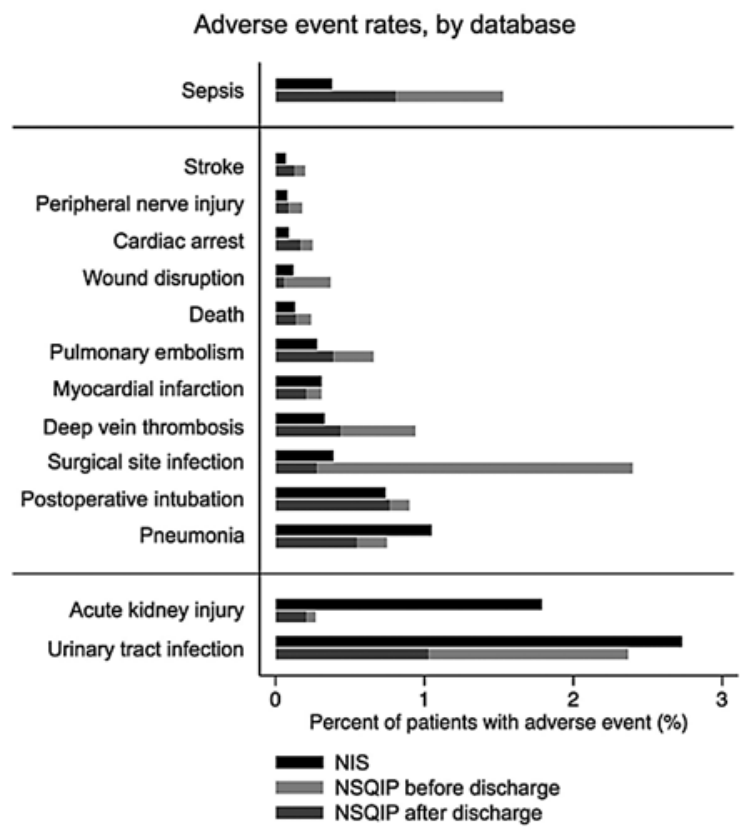

Figure 1. For adverse events shown in the upper third, the rate documented in NSQIP before discharge was more than twice the rate in NIS. For events shown in the lower third, the rate documented in NIS was more than twice the rate documented in NSQIP before discharge. For ocum in events shown in the middle third, the rate documented in NIS and the rate documented in percent of patients with adverse events after discharge can be added to the percent of patients percent of patients with adverse events after discharge can be added to the percent of paten postoperative days. databases in the incidences of three inpatient adverse events: The incidence of sepsis in NSQIP was more than twice that in NIS, and the incidences of acute kidney injury and urinary tract infection in NIS were more than twice those in NSQIP (Figure 1). Eleven other inpatient adverse events had incidences that were within a two-fold difference between the two databases.

Because it does not collect data after patient discharge, NIS does not capture over half of cases of wound disruption, deep vein thrombosis, surgical site infection, and urinary tract infection occurring in the first 30 postoperative days (Figure 1).

Discussion: As database studies become more prevalent in orthopaedic surgery, authors, reviewers, and readers should take caution. This study shows that two commonly used databases can be used to identify similar patients undergoing a common spinal procedure; however, results pertaining to specific comorbidities and adverse events may vary greatly depending on which database is used.

Conclusion: Understanding the inherent differences between databases is crucial to appropriately interpreting database study results.

Poster 3. Range-of-Motion Analysis of Sequential Ponte Osteotomies in a Continuously Loaded Full Thoracic Cadaveric Model with Attached Ribcage

Erin M. Mannen, B.S.; Paul M. Arnold, M.D.; John T. Anderson, M.D.; E. (Lisa) A. Friis, Ph.D.

\section{Kansas University Medical Center}

Disclosures: E.M. Mannen: 1; I; Applied Test Systems, Inc. P.M. Arnold: 4; I; Medtronic Sofamor Danek, Lifespine, Integra Life, Spine Wave, Stryker Spine. 6; I; Z-plasty, Inc.. J.T. Anderson: None. E.A. Friis: 1; I; Applied Test Systems, Inc..

Introduction: Ponte osteotomies (POs) are performed for deformity correction in adolescent idiopathic scoliosis (AIS) and Scheuermann's kyphosis (SK). Using a thoracic cadaveric model with attached ribcage, we quantified flexibility change with sequential POs in all bending modes.

Methods: Five human thoracic cadaveric specimens with intact ribs were dissected. T1 and T12 were potted in a novel six-degree-offreedom testing machine. A pin in T1 tracked ROM. Pure moments were applied at $\mathrm{T} 1$ at $0.5 \%$ second to $\pm 5 \mathrm{Nm}$ for five cycles continuously in lateral bending (LB), flexion-extension (FE), and axial rotation (AR). Each specimen was tested in five sequential conditions: intact, and after one-, two-, three-, and four-level PO. T1-T12 ROM analysis was conducted in three bending modes using the third cycle of the ATS angular displacement data. ROM values were reported as percentage increases.

Results: Significant results were found in FE. Mean percentage increase and degree increase in overall flexibility for one-, two-, three-, and four-level PO were $7.1 \% / 1.35^{\circ}, 8.0 \% / 1.50^{\circ}, 11.0 \% / 2.09^{\circ}$, and $13.5 \% / 2.74^{\circ}$, respectively. Maximum increase in FE flexibility for any one-level PO was $9.2 \% / 1.96^{\circ}$. A four-level PO doubled the sagittal ROM in FE instead of quadrupling it. In AR, there was a trend in ROM increase with more PO levels; the mean percentage increase and degree increase in flexibility after four POs were $4.4 \% / 1.38^{\circ}$. In $\mathrm{LB}$, no significant results were observed.

Discussion: This cadaveric study will contribute to existing knowledge of the mechanical properties of thoracic spine components and how they contribute to overall spinal stability. This information will then be used to create and validate a new analogue non-pathogenic adult spine model with anatomical and biomechanical accuracy, which will be a testing platform for new spinal fusion devices for patients with AIS

Conclusion: Our results support performing POs to increase sagittal flexibility in patients with rigid curves. Lower correction potential for POs was found in FE and insignificant results found in LB and 


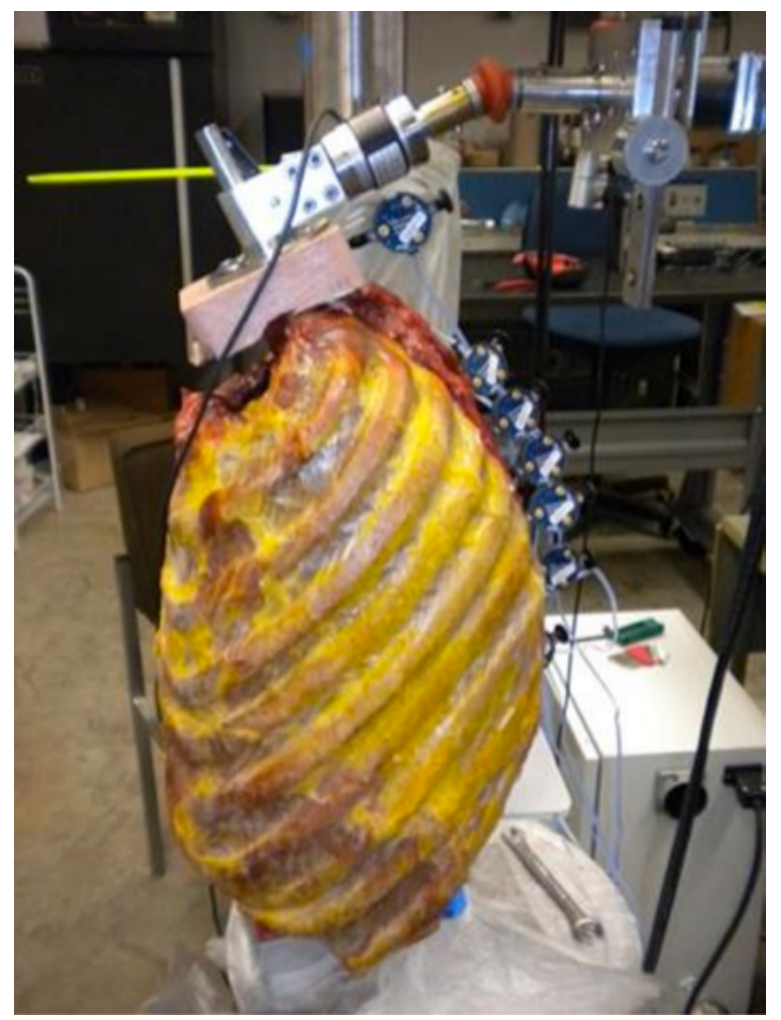

AR, likely due to support by the ribcage. POs influence sagittal flexibility, but the amount of FE correction gained did not equal the ruleof-thumb of $5^{\circ}$ per PO.

Poster 4. Routine Upright Imaging for Evaluating Degenerative Lumbar Stenosis: Incidence of Degenerative Spondylolisthesis Missed on Supine MRI

Brad Segebarth, M.D.; Rick Davis, M.D.

OrthoCarolina Spine Center, Orthopaedic Spine

Disclosures: B. Segebarth: None. R. Davis: 1; I; Stryker Spine. 4; I; Zimmer Spine.

Introduction: Degenerative spondylolisthesis with lumbar stenosis is a well-studied pathology that is most commonly diagnosed by a combination of magnetic resonance imaging (MRI) and upright or flexion-extension radiographs. To our knowledge, there has been no study investigating the incidence of missed diagnosis of anterior spondylolisthesis based on sagittal alignment on MRI. In addition, no study has shown clinical superiority of flexion-extension xrays compared to upright static xrays in order to diagnose a lumbar anterolisthesis.

Methods: We retrospectively evaluated all patients presenting to spine clinic for degenerative lumbar conditions for 24 consecutive months who had an MRI, upright lateral, as well as flexion-extension radiographs at our institution. Comparing radiographic reads by a musculoskeletal radiologist, the incidence of degenerative spondylolisthesis found on dynamic flexion-extension radiographs but not on MRI was determined. We then reviewed each case and compared flexion-extension verses standing lateral views to determine if there was any significant difference in detecting anterolisthesis.

Results: Of 416 patients with eligible studies, 109 were found to have lumbar degenerative spondylolisthesis based on flexion-

\section{Results}

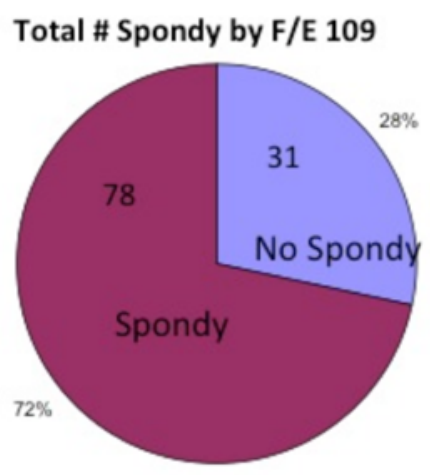

Chart Depicts those dx by MRI

\section{Incidence Missed on MRI 28\%}

extension radiographs. Of these, only 78 were found to have a corresponding spondylolisthesis on MRI, leaving 31/109 (28\%) of lumbar anterior spondylolisthesis levels undiagnosed on MRI. Sensitivity of MRI for detecting spondylolisthesis based on sagittal alignment of $71.6 \%$. No additional anterolisthesis cases were detected on standing flexion-extension verses standing lateral radiographs.

Discussion: This study emphasizes the importance of upright imaging when evaluating lumbar degenerative diseases, as surgical plan may change to include a fusion. Additionally, comparing standing lateral to flexion-extension radiographs indicates that standing lateral $\mathrm{x}$-rays alone may be adequate in detecting degenerative spondylolisthesis.

Conclusions: Routine standing lateral radiographs should be standard practice in order to identify degenerative spondylolisthesis in patients undergoing evaluation for degenerative lumbar disease, as nearly $1 / 3$ of cases will be missed on supine MRI. This may have implications on whether or not an arthrodesis is performed on those patients requiring lumbar decompression.

Poster 5. Risk Factors Associated with Surgical Site Infections Following Lumbar Spine Surgery for Degenerative Pathologies

Alejandro Marquez-Lara, MD; Sreeharsha V. Nandyala, BA; Steven J. Fineberg, MD; Kern Singh, MD

Rush University Medical Center, Orthopaedic Surgery

Disclosures: A. Marquez-Lara: None. S.V. Nandyala: None. S.J. Fineberg: None. K. Singh: 1; I; Pioneer, Stryker, Zimmer. 4; I; Zimmer, Stryker, Globus. 8; I; Vital 5 LLC. 9; I; Thieme, LWW.

Introduction: A surgical site infection (SSI) is one of the most common complications after orthopaedic procedures. The incidence as well as the risk factors associated with a SSI after a lumbar spine surgery (LSS) has been poorly reported. A national surgical database was utilized to analyze preoperative and intraoperative variables in order to determine the risk factors predisposing patients to a SSI following a LSS procedure.

Methods: The National Surgical Quality Improvement Program (NSQIP) database was queried to identify patients who underwent elective LSS between 2006-2011. The resulting cohort was divided 


\section{Abstracts of the 2014 Meeting of the Lumbar Spine Research Society}

\begin{tabular}{|c|c|c|c|}
\hline Variable & $\begin{array}{l}\text { No Wound } \\
\text { Complication }\end{array}$ & $\begin{array}{c}\text { Wound } \\
\text { Complications }\end{array}$ & p-value \\
\hline Count (\%) & $22,214(98.0 \%)$ & $462(2.0 \%)$ & \\
\hline \multicolumn{4}{|c|}{ Demographics } \\
\hline Age (years), mean (SD) & $56.63(15.4)$ & $57.83(15.2)$ & 0.10 \\
\hline$\%$ Female & $48.4 \%$ & $55.5 \%$ & 0.002 \\
\hline \multicolumn{4}{|c|}{ Preoperative Health and Comorbidities } \\
\hline BMI $\left(\mathrm{kg} / \mathrm{m}^{2}\right)$, mean $(\mathrm{SD})$ & $29.92(6.34)$ & $32.68(8.26)$ & $<0.0001$ \\
\hline Diabetes mellitus (\%) & $14.5 \%$ & $23.8 \%$ & $<0.0001$ \\
\hline Smoking & $23.9 \%$ & $23.6 \%$ & 0.89 \\
\hline Alcohol & $3.3 \%$ & $2.8 \%$ & 0.64 \\
\hline COPD & $3.4 \%$ & $4.8 \%$ & 0.12 \\
\hline Cardiac $\mathrm{Hx} / \mathrm{CAD}$ & $9.0 \%$ & $11.2 \%$ & 0.18 \\
\hline Hypertension & $49.5 \%$ & $59.1 \%$ & $<0.0001$ \\
\hline Dialysis & $0.2 \%$ & $0.9 \%$ & $<0.0001$ \\
\hline $\mathrm{Hx}$ of CVA & $2.2 \%$ & $4.4 \%$ & 0.008 \\
\hline Metastatic cancer & $0.1 \%$ & $0.2 \%$ & 0.69 \\
\hline Steroid Use & $3.0 \%$ & $6.1 \%$ & $<0.0001$ \\
\hline \multicolumn{4}{|c|}{ Surgical Statistics and 30-Day Outcomes } \\
\hline Ambulatory Surgery (\%) & $20.0 \%$ & $11.0 \%$ & $<0.0001$ \\
\hline $\begin{array}{l}\text { Operative time (minutes), } \\
\text { mean (SD) }\end{array}$ & $145.8(95.9)$ & $181.6(108.4)$ & $<0.0001$ \\
\hline $\begin{array}{l}\text { No. of Blood transfusions, } \\
\text { mean (SD) }\end{array}$ & $0.145(0.65)$ & $0.469(1.20)$ & $<0.0001$ \\
\hline Resident involvement (\%) & $31.2 \%$ & $40.7 \%$ & $<0.0001$ \\
\hline $\begin{array}{l}\text { Length of stay (days), mean } \\
\text { (SD) }\end{array}$ & $2.57(2.91)$ & $4.51(5.91)$ & $<0.0001$ \\
\hline Readmitted (\%) & $3.9 \%$ & $64.8 \%$ & $<0.0001$ \\
\hline Reoperations (\%) & $1.7 \%$ & $53.1 \%$ & $<0.0001$ \\
\hline Sepsis (\%) & $0.41 \%$ & $19.7 \%$ & $<0.0001$ \\
\hline Mortality (\%) & $0.13 \%$ & $0.43 \%$ & 0.07 \\
\hline \multicolumn{4}{|c|}{ Independent Predictors for Wound Complications } \\
\hline Variable & Odds Ratio & $\begin{array}{c}\mathbf{9 5 \%} \text { Confidence } \\
\text { Interval }\end{array}$ & p-value \\
\hline Female sex & 1.39 & $1.07-1.78$ & 0.01 \\
\hline Obesity (BMI > 30) & 1.81 & $1.39-2.36$ & $<0.0001$ \\
\hline Diabetes & 1.53 & $1.13-2.08$ & 0.006 \\
\hline Dialysis & 4.10 & $1.08-15.5$ & 0.04 \\
\hline Steroid use & 2.03 & $1.23-3.35$ & 0.006 \\
\hline Bleeding disorders & 2.31 & $1.18-4.54$ & 0.02 \\
\hline Resident involvement & 1.64 & $1.27-2.12$ & $<0.0001$ \\
\hline Operative time* & 1.003 & $1.001-1.004$ & $<0.0001$ \\
\hline
\end{tabular}

Poster 6. Oswestry Disability Index: A Psychometric Analysis with 1610 Patients

Darrel S. Brodke, MD; Brandon D. Lawrence; William R. Spiker, MD; Man Hung, PhD

\section{University of Utah, Orthopaedics}

Disclosures: D.S. Brodke: 1; I; Amedica, DePuy Synthes, Medtronic. 4; I; DePuy Synthes. 6; I; Amedica, Vertiflex. B.D. Lawrence: None. W.R. Spiker: None. M. Hung: None.

Background: One fourth of the adult US population has or will experiences back pain and has undergone one of the myriad of treatments. Understanding the outcomes of these many treatments from pharmacologic to surgical, from manipulation to modality, will allow for better understanding and value driven decision-making. Patient reported outcome (PRO) measures are the current standard and include general and disease specific measures. The Oswestry Disability Index (ODI) is the most commonly used disease specific patient reported outcome tool to measure functional disability related to back pain. Few studies have evaluated its psychometric properties in a large patient sample using a modern tool such as the Rasch analysis model. This study aims to identify the benefits and deficiencies of the ODI as an outcome tool for assessing patients with back pain.

Purpose: To investigate the psychometric properties, performance and applicability of the ODI in patients with back pain that visited a University-based outpatient clinic.

Methods: Detailed Rasch analysis of the ODI was performed on 1610 patients visiting an academic spine center. Standard descriptive statistics were also assessed.

Results: The ODI performed well overall. It demonstrated suboptimal unidimensionality (i.e., unexplained variance after accounting for

between patients who had a SSI and those who did not. Preoperative patient characteristics and peri-operative outcomes were assessed. Regression analysis, with a 95\% confidence interval, was performed to determine the risks associated with SSI. Statistical analysis was performed with SPSS v. 20 and a p-value of $<0.001$ denoted significance.

Results: A total of 22,676 lumbar spine surgeries were identified from 2006-2011, of which $462(2.0 \%)$ developed a postoperative SSI. There were no significant differences in patient age between cohorts, however infected patients demonstrated a greater comorbidity burden. Wound complications were also associated with longer operative times, greater resident involvement and greater intraoperative blood transfusions. In addition, the affected cohort demonstrated a longer hospitalization and incurred greater rates of readmissions, reoperations and sepsis. Regression analysis demonstrated that obesity, diabetes, dialysis, steroid use, bleeding disorders, resident involvement, and increased operative times were associated with an increased risk of SSI.

Discussion/Conclusion: This study demonstrates that SSIs are associated with greater peri-operative complications and hospital resource utilization. In addition female gender, obesity, diabetes, dialysis, steroid use, bleeding disorders, resident involvement, and increased operative time were identified to be strongly associated with a SSI. The development of peri-operative protocols based upon these identified risk factors may help mitigate the incidence of SSI and the impact of this occurrence on peri-operative outcomes and hospital resource utilization. the first dimension) of $8.3 \%$. Person reliability was good, at 0.85 and item reliability was excellent, at 1.00 . The overall item fit for the ODI was good with an outfit mean square of 1.02. The ODI had a floor effect of $29.9 \%$ and ceiling effect of $3.9 \%$. The raw score to measure correlation of the ODI was excellent, at 0.944 .

Conclusions: The ODI performed relatively well overall, with some problematic findings. It had good person and item reliability, though it did not demonstrate strong evidence of unidimensionality. The ODI has moderately poor coverage, with a very large floor effect and small ceiling effect, which could present a challenge in interpreting results of scores at the ends of the spectrum.

Poster 7. The Effect of Aging on Healing of Posterolateral Lumbar Fusion in a Rodent Model Using BMP2

Michael D. Daubs, MD; Tetsuo Hayashi; Akinobu Suzuki; Kevin H. Phan; Haijun Tian; Trevor P. Scott; Kunal Sukhija; Bryan Bean; Jeffrey C. Wang, MD

\section{UCLA, Orthopaedic Surgery}

Disclosures: M.D. Daubs: 1; I; Depuy-Synthes. 4; I; Depuy-Synthes. 7; I; Stryker. T. Hayashi: None. A. Suzuki: None. K.H. Phan: None. H. Tian: None. T.P. Scott: None. K. Sukhija: None. B. Bean: None. 
Figure 1. Person-item histogram.

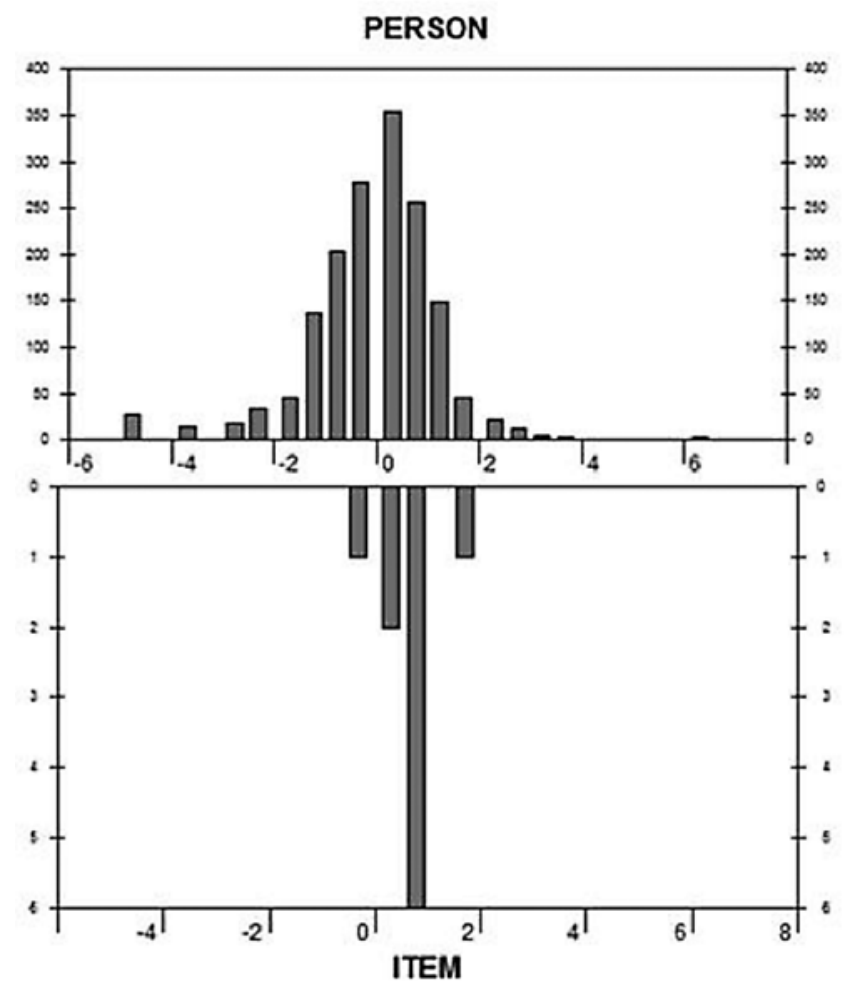

J.C. Wang: 1; I; Biomet, Stryker, Medtronics, Depuy-Synthes, Amedica, Osprey, Aesculap, Seaspine. 6; I; Fziomed, Promethean Spine, Paradigm Spine, Benevenue, NexGen, Pioneer, Amedica, Vertiflex, Electrocore, Surgitech, Axiomed, VG Innovations, Corespine, Expanding Orthopaedics, Syndicom, Osprey, Bone Biologics, Curative Biosciences, Pearldiver.

Introduction: An increased number of lumbar fusions are being performed in older patients. In addition, there has been an increased use of BMP2 to enhance fusion in this patient population. There is very little data in humans evaluating the effect that aging has on overall fusion success as well as the time to fusion. Aging has been shown to delay long bone fracture healing in animal models. The purpose of our study was to evaluate the effect of age on posterolateral fusion using BMP2 in an aged rodent model.

Methods: Twenty male, 8week-old and forty 18-month old Fischer 344 rats underwent bilateral posterolateral intertransverse lumbar fusion at L4-5 using a standard midline approach. The young rats were divided into 2 groups (BMP2 1ug; BMP2 5ug). The older rats were divided into 4 groups (BMP2 1ug sacrificed at 8weeks; BMP2 1ug at 12 weeks; BMP2 5ug at 8weeks; BMP2 5ug at 12 weeks). Fusion was evaluated at 4, 8 , and 12 weeks using Xray and manual palpation at sacrifice.

Results: At 4 weeks, the fusion rate of young/aged rats were $80 \% / 56 \%$ for $\operatorname{lug}(\mathrm{p}=0.01)$, and $100 \% / 58 \%$ for $5 \mathrm{ug}(\mathrm{p}<0.001)$, respectively. At 8 weeks $85 \% / 87 \%$ for 1 ug ( $>00.05)$, and $100 \% / 92 \%$ for 5 ug $(p>0.05)$, respectively. At 12 weeks, $100 \%$ fusion was observed in both groups.

Discussion: Age delays fusion healing time when utilizing BMP2 in a rodent model; however, the ultimate successful fusion rate was similar despite the delayed time to healing in older animals. A higher dose of BMP2 had no effect on healing time in the aged rats.

Conclusion: When compared to younger animals, older animals obtain a successful lumbar fusion, however the time to healing is longer, and a higher dose of BMP2 did not accelerate the healing process.
Poster 8. Predictors of Degenerative Spondylolisthesis and Loading Translation in Surgical Lumbar Spinal Stenosis Patients.

Y. Raja Rampersaud; Rohit Amritanand, MD, FRCS(C); Bheeshma Ravi; Kenny David

University Health Network, Toronto Western Hospital, University of Toronto, Orthopaedics

Disclosures: Y. Rampersaud: None. R. Amritanand: None. B. Ravi: None. K. David: None.

Introduction: The objective of our study is to identify independent patient demographics and radiographic parameters that may predict spondylolisthesis and loading translation in surgical lumbar spinal stenosis (LSS) patients.

Methods: The radiographs and MRIs of 153 consecutive surgical LSS patientsfrom a single surgeon were reviewed. Pelvic incidence (PI), mean facet angle (FA), change in disc height and loading translation (LT - defined as any increase in anterior translation from supine MRI to standing $\mathrm{x}$-ray) were measured from pre-operative MRIs and standing radiographs. Multivariable logistic regression was utilized to determine predictors of spondylolisthesis; $t$-test and chi-squared analyses were used to assess the associations of radiographic variables with LT.

Results: The odds of spondylolisthesis were significantly higher in females (adjusted OR [95\% CI] = $2.56[1.19,5.63], \mathrm{p}=0.017$ ), and in patients with higher PI: each additional degree of PI was associated with odds of spondylolisthesis $1.05(95 \% \mathrm{CI}=[1.02,1.08])$ times as great. The odds were significantly lower with increasing (i.e. more horizontal) mean FA (OR = 0.95[0.92,0.99], p = 0.006). Age, BMI and disc height were not significantly associated with odds of spondylolisthesis. Among patients with spondylolisthesis, LT was associated with a higher PI: $59 \pm 15$ vs. $53 \pm 13$ degrees ( $\mathrm{p}=0.02$ ); a higher PI was also marginally significant in those with $>3 \mathrm{~mm}$ of LT ( $\mathrm{p}=0.06$ ); facet angle and disc height were not significantly associated with LT.

Conclusions: This study indicates that women with higher PI and lower FA have a greater risk of spondylolisthesis, and that a higher pelvic incidence may predict instability in patients with spondylolisthesis. Based on these findings we hypothesize that PI alone or in combination with other anatomical factors, may play an important role in surgical decision making (e.g. to fuse or not to fuse) for degenerative spondylolisthesis and warrants further investigation.

Poster 9. Does Pedicle Fill Affect Pull Strength After Rod Reduction During Scoliosis Surgery?

William F. Lavelle; Nathaniel R. Ordway, MS, PE; Brandon Clair; Stephen A. Albanese, MD

\section{SUNY, Department of Orthopedics}

Disclosures: W.F. Lavelle: 2; I; Stryker. 7; I; DePuy, A Johnson \& Johsnon Company. N.R. Ordway: None. B. Clair: None. S.A. Albanese: 6; I; Bristol-Myers Squibb, Eli Lilly, Merck, Procter \& Gamble.

Introduction: The concept of reducing a deformed spine to a precontoured rod as a scolosis treatment has become popular. This has led to development of rod reducing devices that apply a tensile force to pedicle screw to reduce the deformed spine to a pre-contoured rod. However, pull-out strength of screws may be affected by these maneuvers. Other studies found pedicle screw size and fill significantly affect pull-out strength. The purpose of this study was to determine the effect of pedicle screw size and fill during a simulated surgical rod reduction procedure.

Methods: Spine specimens, T1-L3, were dissected from four 


\section{Abstracts of the 2014 Meeting of the Lumbar Spine Research Society}

cadavers. Specimens underwent helical $1 \mathrm{~mm}$ CT scanning to evaluate pedicle cross-section in coronal and transverse planes to determine minimal pedicle diameter. Screws were placed and imaged to confirm placement. Screw holes were tapped a millimeter smaller than assigned screw diameter using a single lead tap set.

At odd vertebral levels, pedicle screws of approximately $80 \%$ fill were placed bilaterally. At even vertebral levels, pedicle screws were placed bilaterally, one with $>80 \%$ fill and one with $<80 \%$ fill. A rod was instrumented across even vertebral levels and fixed with caps at odd levels. The rod was contoured over even levels to have $5 \mathrm{~mm}$ residual gap between rod and tulip. A rod reduction device was used to engage the rod into pedicle screw at even levels. After 5 minute period to allow for stress relaxation of rod-screw construct, the rods were removed.

The pedicle screw-bone construct was tested at a tension rate of $5 \mathrm{~mm} / \mathrm{min}$. Load and displacement data were collected at $100 \mathrm{~Hz}$ and peak pull-out force measured in Newtons. All of the even vertebral levels were grouped to examine pedicle fill and rod reduction on pullout force utilizing a t-test.

Results: The average pull out for $>80 \%$ pedicle fill was $553 \mathrm{~N} \pm$ 272 and $<80 \%$ pedicle fill was $581 \mathrm{~N} \pm 404$. There was no significant difference in pull-out force based on pedicle fill $<80 \%$ compared to $>80 \%(\mathrm{p}>.05)$.

Discussion: Our study showed that there was no significant differences in pull-out force based on pedicle fill during simulated surgical rod reduction procedures.

Conclusion: Pedicle fill does not appear to reduce the effects of rod reduction of rod pull-out.

\section{Poster 10. Perioperative Outcomes of Multi-level versus Single} Level Lumbar Spine Surgery

Sreeharsha V. Nandyala, BA; Alejandro Marquez-Lara, MD; Steven J. Fineberg, MD; Kern Singh, MD

\section{Rush University Medical Center, Orthopaedic Surgery}

Disclosures: S.V. Nandyala: None. A. Marquez-Lara: None. S.J. Fineberg: None. K. Singh: 1; I; Pioneer, Stryker, Zimmer. 4; I; Zimmer, Stryker, Globus. 8; I; Vital 5 LLC. 9; I; Thieme, LWW.

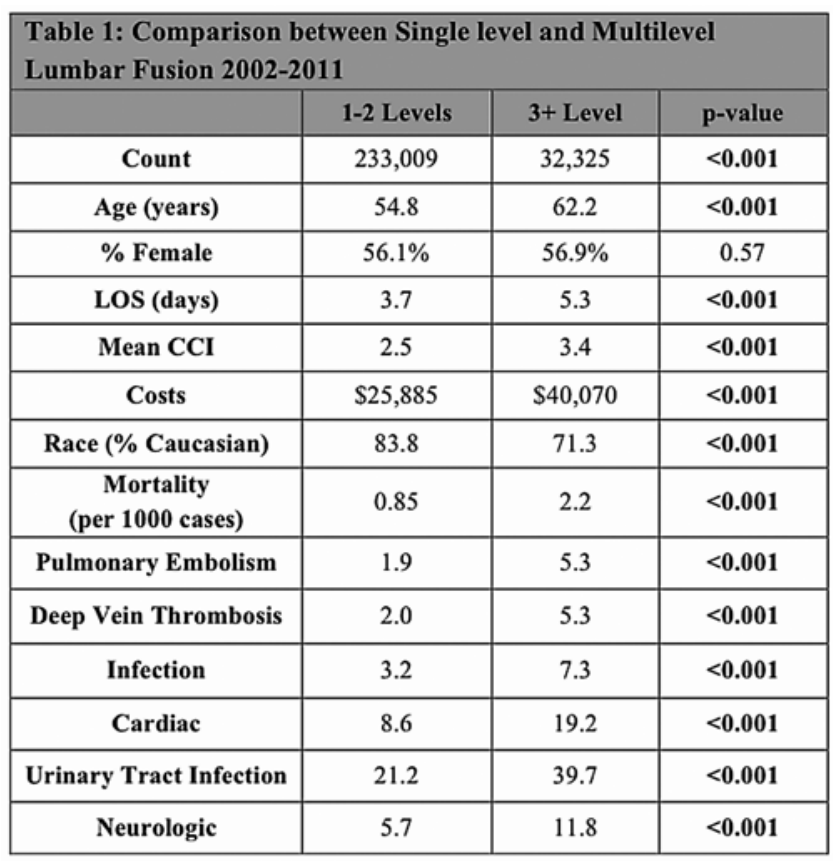

Introduction: Lumbar fusion (LF) is commonly performed for both single- and multi-level degenerative pathology. The surgical outcomes may vary based upon the complexity of the LF. To characterize these differences, a population-based database was analyzed with regards to the patient demographics, complications, costs, and mortality.

Methods: Data from the Nationwide Inpatient Sample was queried from 2002-2011. Patients undergoing a LF were identified and separated into two cohorts: 1-2 level and 3+ level fusions. Patient demographics, comorbidities (CCI), length of stay, costs, and perioperative outcomes were assessed. Multinomial regression analysis, with a 95\% confidence interval, was utilized to demonstrate independent predictors of in-hospital mortality after LF. Statistical analysis was performed with $\chi 2$-tests and independent sample T tests with a $\mathrm{p}$-value of $<0.001$ to denote significance.

Results: A total of 265,334 lumbar fusions were identified in the NIS of which 233,009 (87.8\%) patients underwent a 1-2 level fusion and $32,325(12.2 \%)$ underwent a $3+$ level fusion. The $3+$ level fusion cohort was significantly older $(62.2$ vs 54.8 years; $p<0.001)$ and demonstrated a greater comorbidity burden than the 1-2 level fusion group (CCI 3.4 vs $2.5 ; \mathrm{p}<0.001$ ). In addition, patients requiring $3+$ level fusion incurred a significantly longer hospitalization (5.3 vs 3.7 days; $p<0.001)$ and greater total hospital costs $(\$ 40,070$ vs $\$ 25,885$; $\mathrm{p}<0.001)$. Furthermore, the $3+$ level fusion cohort demonstrated a significantly greater incidence of pulmonary embolism (PE), deep vein thrombosis (DVT), wound infection, cardiac and neurological complications, and urinary tract infections (UTI) $(<0.001)$. Mortality was significantly greater in the $3+$ level fusion group. Lastly, regression analysis demonstrated that age $>65$ years, coagulopathy, electrolyte disturbances, and pulmonary disorders were significant predictors of in hospital mortality after a lumbar fusion.

Discussion/Conclusion: Patients undergoing a multilevel LF demonstrated a longer hospitalization, greater hospital costs, and incurred an increased incidence of postoperative complications and mortality. These findings are likely due to an older and more comorbid patient population and the greater complexity associated with a $3+$ level lumbar fusion. Further studies are warranted to help mitigate these risk factors and to account for the greater incidence of postoperative complications after multilevel LF surgery.

Poster 11: XLIF vs MAS TLIF for the Treatment of Degenerative Spondylolisthesis: Interim Results from an Ongoing Prospective Multi-Center Comparative Study

Jonathan Sembrano; Antoine Tohmeh, M.D.; Robert E. Isaacs, M.D.; SOLAS Degenerative Study Group

\section{University of Minnesota, Orthopaedic Surgery}

Disclosures: J. Sembrano: 7; I; NuVasive, Inc. A. Tohmeh: 1; I; NuVasive, Inc.. 2; I; NuVasive, Inc.. 4; I; NuVasive, Inc.. 6; I; Amedica. R.E. Isaacs: 1; I; NuVasive. S. Degenerative Study Group: 1; I; NuVasive, Inc.. 2; I; NuVasive, Inc.. 3; I; NuVasive, Inc.. 4; I; NuVasive, Inc.. 5; I; NuVasive, Inc.. 6; I; NuVasive, Inc.. 7; I; NuVasive, Inc.. 8; I; NuVasive, Inc..

Introduction: This prospective, multicenter, IRB-approved study aims to compare indirect decompression via XLIF and direct posterior decompression via TLIF for the treatment of low-grade spondylolisthesis with a specific interest in clinical and radiographic results. This abstract serves as an interim report of the 6 month outcomes.

Methods: Adult patients with Grade I-II degenerative spondylolisthesis at one or two lumbar levels were treated with either MAS TLIF or XLIF. Motor/sensory evaluations were conducted by the treating physician. Patient reported outcomes and radiographic measurements were also collected pre- and post-op. 


\section{Abstracts of the 2014 Meeting of the Lumbar Spine Research Society}

Results: At the time of analysis 48 patients (25 XLIF, 23 TLIF) out of 55 enrolled had completed 6-month follow-up, and early postop neural deficit data was available for 55 patients (29 XLIF, 26 TLIF). Pre-op neural deficits resolved after surgery in $100 \%$ of XLIF and $73 \%$ of TLIF patients. Mean surgery times favored XLIF (171 min vs $186 \mathrm{~min}) .79 \%$ of XLIF and $27 \%$ of TLIF patients lost less than $100 \mathrm{~mL}$ blood $(\mathrm{p}<0.01)$. There were 3 dural tears in the TLIF group and none in the XLIF group. Mild transient post-op hip flexion weakness presented in $34 \%$ of XLIF patients, which all resolved by 6 months. 1/29 XLIF patients had new distal motor deficits noted postoperatively, which resolved by 6 months. New post-op sensory deficits were noted in 2/29 XLIF and 2/26 TLIF patients, which all resolved. Post-op VAS and ODI scores were similar between groups, and both showed significant improvement over baseline.

XLIF resulted in less segmental motion and less early post-op subsidence. Postoperative lumbar lordosis, approach-side foraminal dimensions, and reduction of spondylolisthesis were similar between treatment groups. At 3 months contralateral foraminal height was significantly greater than approach-side foraminal height within the TLIF group only.

Conclusion: In this early interim analysis comparing XLIF to TLIF for the treatment of low-grade degenerative spondylolisthesis, only subtle differences were identified. Patient-reported pain, function, and satisfaction are comparable. Continued study follow-up is required to make stronger comparisons between procedures.

Poster 12. Iatrogenic Impaction Damage with a Static and Expanding Lateral Interbody Spacer Using a Foam Block Vertebral Body Model in Assessing Intraoperative Subsidence

William Hunter, M.D.; Mark Moldavsky, M.S.; Brandon Bucklen, $\mathrm{PhD}$.

\section{Globus Medical, Research}

Disclosures: W. Hunter: 4; I; Globus Medical Inc., Nuvasive Inc., CNK Group. 8; I; Globus Medical Inc., Nuvasive Inc. M. Moldavsky: 3; I; Globus Medial Inc.. 6; I; Globus Medical Inc. B. Bucklen: 3; I; Globus Medical Inc.. 6; I; Globus Medical Inc..

Introduction: Lateral interbody fusion approaches have been gaining traction, particularly based on the ability to indirectly decompress. The authors investigate intraoperative subsidence caused by iatrogenic damage to the cortices in the process of distracting the disc space, using either a static or vertically-expanding lateral device.

Methods: Rectangular foam blocks (Sawbones Inc., Vashon, Washington) made from 15 PCF with a $2 \mathrm{~mm}$ layer of 40 PCF were used to model vertebral bodies. The blocks were placed inside a metal fixture that allows for adjustable resistive forces, simulating spine tension in the lateral position.

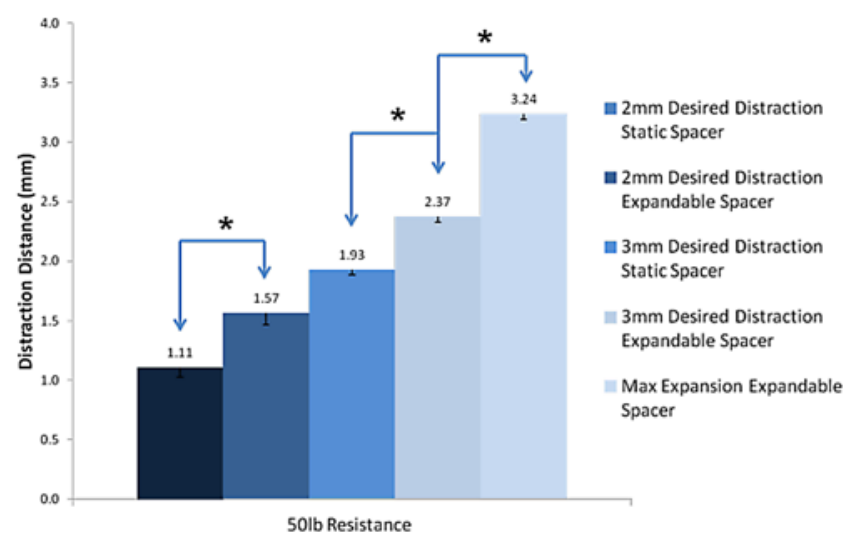

In Group 1,2mm and 3mm interference was created between the blocks and CoRoent ${ }^{\circledR}$ (Nuvasive Inc., San Diego, CA) static spacer. Interference is the final height of the spacer minus the opening between the blocks. CoRoent ${ }^{\circledR}$ was impacted between the foam blocks and the distance between the blocks was measured after insertion. In Group 2, CALIBER®-L (Globus Medical Inc., Audubon, PA), a vertically expandable interbody spacer, was impacted at the smallest height, matching the disc space clearance, and expanded to the height needed to fit the disc space $(2 \mathrm{~mm}$ or $3 \mathrm{~mm}$ interference, and maximum expansion). This was repeated for a total of five data points at, physiological, 50lb of resistance. To determine statistical significance, a one way ANOVA and post hoc Student Newman-Keuls test were performed $(\mathrm{p} \leq 0.05)$.

Results: With 50lb of resistance and $2 \mathrm{~mm}$ of interference, static and expandable spacers provided $1.11 \pm 0.08 \mathrm{~mm}$ and $1.57 \pm 0.10 \mathrm{~mm}$ of distraction, respectively. At $3 \mathrm{~mm}$ of interference, static and expandable spacers provided $1.93 \pm 0.04 \mathrm{~mm}$ and $2.37 \pm 0.11 \mathrm{~mm}$, respectively. At maximum expansion, the expandable spacer provided $3.24 \pm 0.05 \mathrm{~mm}$ of distraction. There was a significant difference between static and expandable spacers at both $2 \mathrm{~mm}$ and $3 \mathrm{~mm}$ of interferance. Distraction of the expandable spacer at maximum expansion is significantly greater compared to the expandable spacer at $3 \mathrm{~mm}$ expansion.

Conclusion: An expandable lateral interbody spacer resulted in significantly more distraction than a static spacer at both 2 and $3 \mathrm{~mm}$ of interference. Necessarily, this resulted in less endplate damage in the simulated bone model, which may correlate to less intraoperative subsidence.

Poster 13. Biomechanical Effects of Thoracolumbar Burst Fracture Stability with In Vitro Human Cadaveric Model Using Traditional and Integrated Expandable Corpectomy Spacers

Ripul Panchal; Erika Matheis, M.S.; Manasa Gudipally, M.S.; Kanaan Salloum, B.S.; Mir Hussain, B.S.; Kee Kim, M.D.; Brandon Bucklen, Ph.D.

\section{Globus Medical}

Disclosures: R. Panchal: None. E. Matheis: 3; I; Globus Medical. M. Gudipally: 3; I; Globus Medical. K. Salloum: 3; I; Globus Medical. M. Hussain: 3; I; Globus Medical. K. Kim: 4; I; Stryker. 7; I; Asubio, Globus Medical, Lanx, LDR. 8; I; Globus Medical. B. Bucklen: 3; I; Globus Medical.

Introduction: Traditional corpectomy reconstruction for burst fractures involves anterior column support with posterior internal fixation at one or two levels cephalad/caudal. Integrated fixation corpectomy spacers have been developed to further add anterior support and potentially replace anterior column plating. This study evaluated the biomechanical effect of implant footprint size as well as effect of integrated screws.

Methods: Six fresh frozen human thoracolumbar spines (T11-L3) were tested on a six-degrees-of-freedom simulator enabling unconstrained motion $(\mathrm{ROM})$ in flexion-extension $(\mathrm{FE})$, lateral bending (LB) and axial rotation (AR), following simulated burst fracture at L1. Expandable titanium cages with/without integrated screws (Fi)/(F) (FORTIFY® Integrated/FORTIFY®), Globus Medical, Audubon, PA) were tested with small endplates (s) $(21 \times 23 \mathrm{~mm})$ and large endplates (L) $(22 \times 40-50 \mathrm{~mm})$. Posterior instrumentation (PI) (REVERE®, Globus Medical, Audubon, PA) was used one level above/below burst fracture. Lateral plate (LP) (TRUSS ${ }^{\circledR}$, Globus Medical, Audubon, PA) fixation was tested. Devices were tested in order: 1). Pre-operative; 2)Fis+PI; 3)Fs+PI ; 4)Fs+PI+LP; 5) FL+PI+LP; 6)FL+PI; 7)FiL+PI.

Results: Significant decrease $(\mathrm{p}<0.01)$ in ROM is seen from intact in FE and LB to integrated screw and lateral plate with PI (Fis+PI; FiL+PI; Fs+PI+LP; FL+PI+LP). A small trend in LB showed that LP 


\section{Abstracts of the 2014 Meeting of the Lumbar Spine Research Society}

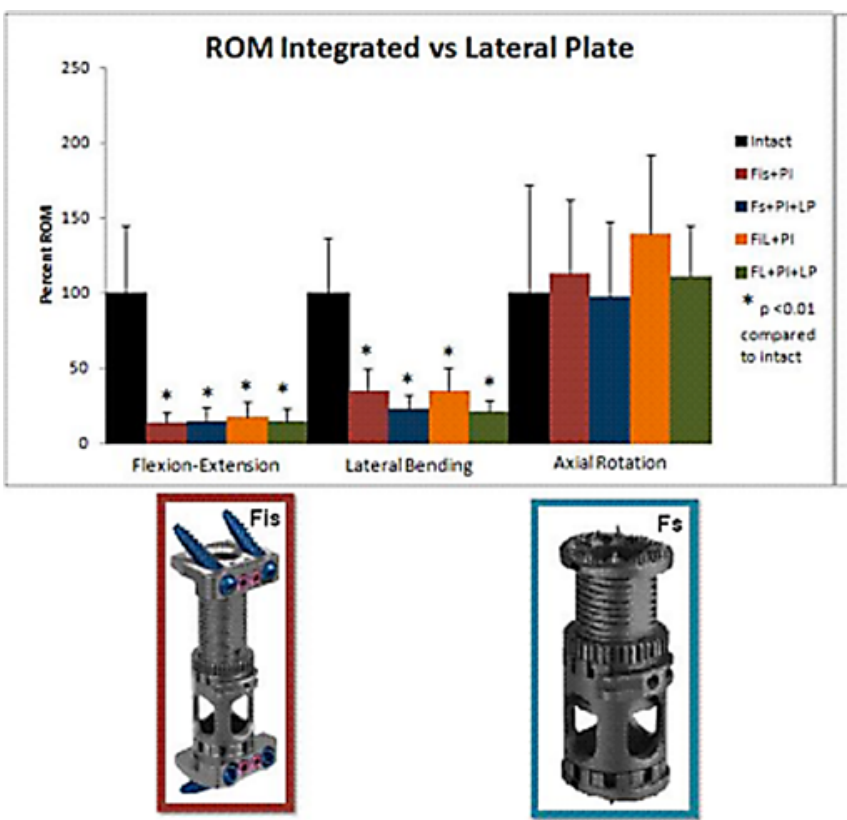

provides more fixation than integrated screws, however no significant differences were found between integrated screws and lateral plate within ROM testing. Footprint sizing showed no significant differences in small versus large footprints, however significant decrease in ROM $(\mathrm{p}<0.01)$ is reached against intact in FE and LB. AR failed to reach significance under both test conditions.

Conclusions: Expandable corpectomy spacers with integrated screws show biomechanical similarity to lateral plating with posterior instrumentation while providing vertebral fixation. Footprint sizing does not affect biomechanical performance of ROM, however, larger footprints may have clinical use in preventing subsidence when resting upon the apophyseal ring.

Poster 14. Lumbar Spine Fractures in Frontal Impact Crashes: An Increasing Concern?

Jamie L. Baisden, MD; Narayan Yoganandan, PhD; Greg Stadter; Dale Halloway; Frank Pintar, PhD

\section{Medical College of Wisconsin, Neurosurgery}

Disclosures: J.L. Baisden: None. N. Yoganandan: None. G. Stadter: None. D. Halloway: None. F. Pintar: 7; I; DOT.

Introduction: Compression-related thoracolumbar fractures occur in frontal impacts and yet the mechanism of injury is poorly understood. The objective of this investigation was to characterize these injuries using real world crash data from the US-DOT-NHTSA NASS-CDS and CIREN databases.

Methods: Thoracic and lumbar vertebral body fracture codes were searched for in the two databases. The National Automotive Sampling System (NASS) database was used to characterize population trends as a function of crash year and vehicle model year. The Crash Injury Research and Engineering Network (CIREN) database was used to examine a case series in more detail.

Results: From the NASS database there were 2000-4000 occupants in frontal impacts with thoracic and lumbar vertebral body fractures per year. There was an increasing trend in incidence rate of thoracolumbar fractures in frontal impact crashes as a function of vehicle model year from 1993 to 2012; this was not the case for other crash types. From the CIREN database, the thoracolumbar spine was most

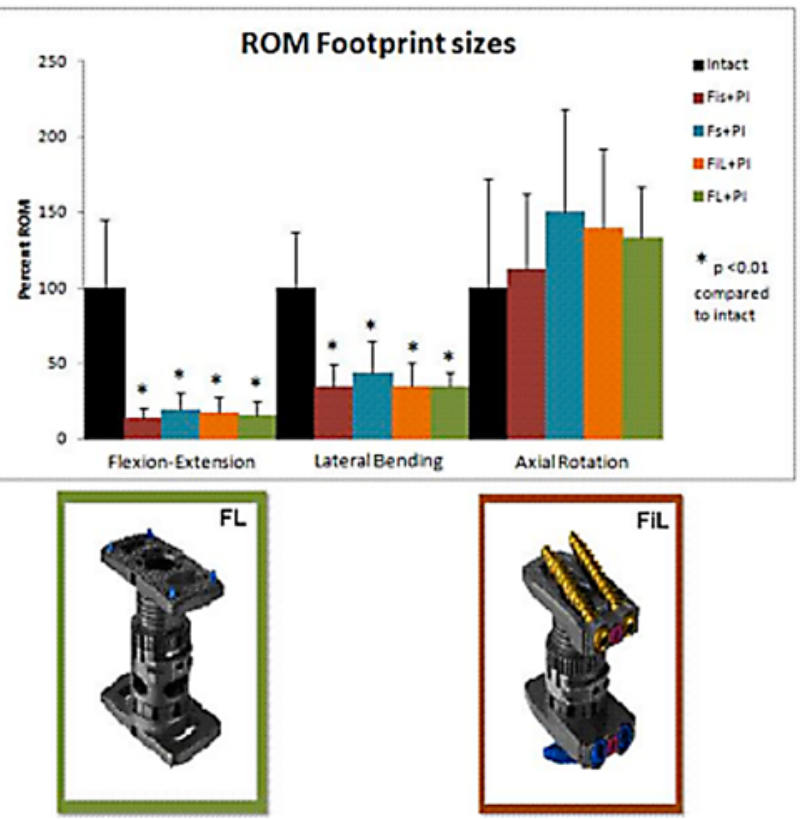

commonly fractured at either the T12 or L1 level. Major, burst type fractures occurred predominantly at T12, L1 or L5. About half the time, the vehicle collision occurred on the roadway and approximately $40 \%$ occurred with impacts to a tree/pole. $70 \%$ of impacts occurred below regulatory crash speeds.

Conclusions: The data from real world crashes suggest that thoracolumbar fractures are increasing and are related to the vehicle seat structure, restraint systems, and object struck suggesting that the crash deceleration pulse may influence the compression vector that migrates up the spinal column. Smaller, stiffer car structures may be contributing to the increased occurrence.

Poster 15. Comparative Analysis of Cauda Equina Syndrome (CES) Patients versus an Unaffected Population Undergoing Spinal Surgery

Bryan J. Marascalchi, BS; Peter G. Passias, MD; Vadim Goz, BA; Jeffrey H. Weinreb, BS; LiJin Joo, MA; Thomas J. Errico, MD

Disclosures: B.J. Marascalchi: None. P.G. Passias: None. V. Goz: None. J.H. Weinreb: None. L. Joo: None. T.J. Errico: 1; I; K2M. 2; I; K2M, Depuy. 6; I; Fastenetix. 7; I; Fridolin Trust, Paradigm Spine, Orthopedic Research and Education FDN (OREF), AOSpine, Orthopedic Medical Education Grant Assoc. (OMEGA).

Introduction: Determine patient demographics, incidence of comorbidities and procedure-related complications, and identify risk factors for morbidity and mortality after spinal surgery for cauda equina syndrome (CES). No study has provided nationwide estimates of patient characteristics and procedure-related complication rates after spinal surgery for CES relative to an unaffected population.

Methods: Nationwide Inpatient Sample data collected from 20012010 was analyzed. Discharges with lumbar spinal fusion, decompression, or discectomy were included. The CES cohort included diagnoses of CES; the unaffected cohort included lumbar spinal pathology diagnoses. Patient demographics, incidence of comorbidities and procedure-related complications, and risk factors for morbidity and mortality were compared.

Results: Discharges for 11,207 CES and 689,799 unaffected patients were identified. Differences between cohorts were found for demographic and hospital data. Average comorbidity indices for the 


\section{Abstracts of the 2014 Meeting of the Lumbar Spine Research Society}

CES cohort were found to be increased ( 0.23 vs.0.13, $\mathrm{p}<0.0001)$, and the incidence of total procedure-related complications (18.63\% vs. $13.12 \%, \mathrm{p}<0.0001)$. In-hospital mortality rate was increased for the CES cohort $(0.30 \%$ vs. $0.08 \%, \mathrm{p}<0.0001)$. Numerous comorbidities associated with additional risk for morbidity and mortality among the CES cohort were identified.

Conclusion: Relative to an unaffected population undergoing similar treatment, CES patients were more likely to have increased associated comorbidities on presentation, increased complication rates, and a prolonged hospital course postoperatively. CES was found to carry an increased incidence of procedure-related complications and in-hospital mortality. A number of comorbidities associated with additional risk for morbidity and mortality among the CES cohort were identified. This study provides clinically useful data to educate patients at risk and direct future research.

Poster 16. Single-level Lateral Lumbar Interbody Fusion for the Treatment of Adjacent Segment Disease: A Retrospective Twocenter Study

Alexander Aichmair, MD; Marjan Alimi, MD; Alexander P. Hughes, MD; Jennifer Shue, MS; Andrew A. Sama, MD; Jerry Y. Du, BS; Roger Hartl, MD; Frank P. Cammisa, MD; Federico P. Girardi, MD

\section{Hospital for Special Surgery}

Disclosures: A. Aichmair: None. M. Alimi: None. A.P. Hughes: 4; I; NuVasive, Inc.. J. Shue: None. A.A. Sama: 1; I; DePuy Spine, Ortho Development Corp.. 4; I; DePuy Spine, LifeSpine, Ortho Development Corp., RTI Biologics, Inc., Spineview. 6; I; Paradigm Spine, LLC, Small Bone Innovation, LLC. J.Y. Du: None. R. Hartl: 4; I; DePuy Synthes, Brainlab, LANX, AOSpine. 7; I; Baxter. F.P. Cammisa: 1; I; NuVasive, Inc.. 4; I; Alphatec Spine, Inc., Centinel Spine, Inc., DePuy Spine, Vertebral Technologies, Inc., Healthpoint Capital Partners, LP, Ivy Healthcare Partners, LP, Mazor Surgical Technologies, NuVasive Inc., Paradigm Spine, LLC, Spinal Kinetics, Viscogliosi Brothers, LLC. 6; I; Alphatech Spine, Inc., BI Members, LLC, Disc Motion Technologies, Inc., Healthpoint Capital Partners, LP, Ivy Healthcare Partners, LP, NuVasive, Inc., Orthopedic Investment Partners, LP, Paradigm Spine, LLC, Small Bone Innovations, Spinal Kinetics, Viscogliosi Brothers, LLC, Centinal Spine, Inc., K2 Medical, LLC, Knee Creations, Scient'x USA, Vertebral Technologies. F.P. Girardi: 1; I; DePuy Spine, Ortho Development Corp., NuVasive, Inc.. 4; I; DePuy Spine, SpineArt, Centinel Spine, Ethicon, LANX, Inc., NuVasive, Inc., Ortho Development Corp., LDR Spine, Inc., Orthogem, Spineart USA, Spineview, Wenzel Spine, Inc.. 6; I; LifeSpine, Paradigm Spine, LLC, Pioneer Surgical Technology, LLC, Small Bone Innovations.

Introduction: The aim of the present study was to assess the surgical outcome and reoperation rate in patients who underwent single-level lateral lumbar interbody fusion (LLIF) for the treatment of adjacent segment disease (ASD).

Methods: Patients who had undergone LLIF at two institutions between 03/2006 and 04/2012 were included, if the following criteria were met: (1) single-level LLIF for the treatment of ASD, and (2) no prior instrumentation at the index LLIF level. The medical records, operative reports, radiographic imaging studies, and office records of included patients were retrospectively reviewed.

Results: Out of 523 patients who had undergone LLIF, 10.1\% $(n=53)$ met the inclusion criteria, and were post-operatively followed for an average of 16.1 \pm 9.7 months (range: 5-44). Single-level LLIF was performed adjacent to an average of $2.3 \pm 1.1$ previously treated levels (range: 1-4). The severity of back and leg pain at the most recent follow-up was reported as $4.1 \pm 3.2$ (range: $0-10$ ) and $3.3 \pm 3.3$ (range: 0-10), respectively, which were significantly lower than preoperative scores $($ each $\mathrm{p}<0.001)$. Comparing pre- versus the most recent post-operative radiographs, after a radiographic follow-up of 18.2 \pm 12.8 months (range: $2.5-50.9$ ), there was an increase in lumbar lordosis by $-1.0 \pm 8.9$ degrees (range: $-20.5-17.6 ; \mathrm{p}=0.436$ ) and segmental lordosis by $-2.6 \pm 6.9$ degrees (range: $-18.7-9.8 ; \mathrm{p}=0.011$ ); the implantation of the cages resulted in a reduction of lumbar coronal angulation by $-0.4 \pm 2.8$ degrees (range: $-8.2-6.4 ; \mathrm{p}=0.356$ ), and segmental coronal angulation by $-1.3 \pm 2.8$ degrees (range: $-8.4-6.3$; $\mathrm{p}=0.002)$. The increase in intervertebral height was $3.6 \pm 2.8 \mathrm{~mm}$ (range: -1.9-9.5), compared to the pre-operative setting $(\mathrm{p}<0.001)$. The rate of add-on surgery related to the index LLIF procedure for ASD was $20.8 \%(n=11)$, which was performed after an average of 14.6 \pm 10.1 months (range: 3.3-31.0).

Discussion: Single-level LLIF for the treatment of ASD resulted in a significant reduction in pain severity, increase in segmental lordosis, decrease in segmental coronal angulation, and increase in intervertebral height at the surgical level.

Conclusions: According to the results of the present study, singlelevel LLIF is an effective surgical treatment option for ASD after spinal arthrodesis both with regard to the clinical as well as the radiographic outcome in a large proportion of cases (79.2\%).

Poster 17. Incidence, Predictors and Outcomes of Pulmonary Embolism after Lumbar Spine Surgery

Alejandro Marquez-Lara, MD; Sreeharsha V. Nandyala, BA; Steven J. Fineberg, MD; Kern Singh, MD

\section{Rush University Medical Center, Orthopaedic Surgery}

Disclosures: A. Marquez-Lara: None. S.V. Nandyala: None. S.J. Fineberg: None. K. Singh: 1; I; Pioneer, Stryker, Zimmer. 4; I; Zimmer, Stryker, Globus. 8; I; Vital 5 LLC. 9; I; Thieme, LWW.

Introduction: Postoperative pulmonary embolism (PE) is a well-

\begin{tabular}{|c|c|c|c|}
\hline Variable & No PE & PE & p-value \\
\hline \multirow{2}{*}{ Count (\%) } & $22,531(99.7 \%)$ & $79(0.3 \%)$ & \\
\hline & $56.66(15.4)$ & $63.97(12.4)$ & $<0.0001$ \\
\hline $\begin{array}{l}\text { Age (years), mean (SD) } \\
\% \text { Female }\end{array}$ & $48.6 \%$ & $41.8 \%$ & 0.23 \\
\hline \multicolumn{4}{|c|}{ Pre-operative Health and Co-morbidities } \\
\hline BMI $\left(\mathrm{kg} / \mathrm{m}^{2}\right)$, mean (SD) & $29.97(6.38)$ & $33.41(8.46)$ & $<0.0001$ \\
\hline \multirow{2}{*}{$\begin{array}{l}\text { Diabetes mellitus (\%) } \\
\text { Smoking (\%) }\end{array}$} & 14.7 & 19.0 & 0.28 \\
\hline & 23.9 & 3.8 & $<0.0001$ \\
\hline Alcohol (\%) & $\frac{23.9}{32}$ & 2.0 & 0.62 \\
\hline COPD (\%) & $\frac{3.2}{3.4}$ & 7.6 & 0.04 \\
\hline Cardiac history / CAD (\%) & 9.1 & 6.0 & 0.45 \\
\hline \multirow{2}{*}{$\begin{array}{l}\text { Hypertension (\%) } \\
\text { Peripheral vascular discase (\%) }\end{array}$} & 49.7 & 64.6 & 0.009 \\
\hline & 1.0 & 2.0 & 0.48 \\
\hline Dialysis (\%) & 0.2 & 0.0 & 0.72 \\
\hline History of CVA (\%) & 2.2 & 0.0 & 0.29 \\
\hline Paralysis (\%) & \multirow{2}{*}{$\begin{array}{l}4.7 \\
0.1\end{array}$} & 8.0 & 0.28 \\
\hline Metastatic cancer (\%) & & 1.3 & 0.009 \\
\hline Open wound/ wound infection (\%) & 0.6 & 2.5 & 0.03 \\
\hline Steroid use (\%) & 3.0 & 8.9 & 0.002 \\
\hline Pre & ative Laboratory $\mathbf{V}$ & lues & \\
\hline WBC, mean (SD) & $\begin{array}{l}7.53(2.44) \\
\end{array}$ & $8.21(2.92)$ & 0.02 \\
\hline Hematocrit, mean (SD) & $40.96(4.26)$ & $40.95(3.80)$ & 0.99 \\
\hline Platelets, mean (SD) & $247.5(67.5)$ & $257.4(75.8)$ & 0.20 \\
\hline Creatinine, mean (SD) & $0.937(0.47)$ & $1.048(1.10)$ & 0.05 \\
\hline Serum albumin, mean (SD) & $4.14(0.44)$ & $3.93(0.40)$ & 0.003 \\
\hline INR, mean (SD) & $1.019(0.24)$ & $1.196(1.13)$ & $<0.0001$ \\
\hline Surgical Statistics : & 0-Day Outcomes w & $\mathrm{h}$ and without a & \\
\hline Ambulatory Surgery (\%) & 19.9 & 7.6 & 0.006 \\
\hline Operative time, mean (SD) & $146.2(95.9)$ & $211.7(154.2)$ & $<0.0001$ \\
\hline No. of blood transfusions, mean (SD & $0.152(0.67)$ & $0.450(1.79)$ & 0.05 \\
\hline Resident involvement (\%) & 31.4 & 32.0 & 0.93 \\
\hline Length of stay (days), mean (SD) & $2.60(2.99)$ & $6.47(6.32)$ & $<0.0001$ \\
\hline Readmitted (\%) & 4.9 & 47.5 & $<0.0001$ \\
\hline Deep vein thrombosis (\%) & 0.5 & 27.9 & $<0.0001$ \\
\hline Mortality (\%) & \begin{tabular}{|l|l|} 
& 0.1 \\
\end{tabular} & 5.1 & $<0.0001$ \\
\hline & ndent Predictors for & & \\
\hline Obesity (BMI > 30) & \begin{tabular}{|l|l|}
1.67 & \\
\end{tabular} & $(1.02 \cdot 2.72)$ & 0.04 \\
\hline Smoking & 0.15 & $0.05 \cdot 0.49$ & 0.002 \\
\hline Elevated WBC & 1.91 & $1.04-3.50$ & 0.04 \\
\hline Operative time* & 1.004 & $1.002-1.006$ & $<0.0001$ \\
\hline $\begin{array}{l}\text { PE: Pulmonary embolism; SD: Standard D } \\
\text { Coronary Artery Disease; TIA: Transient } \\
\text { count; INR: International normalized ratio } \\
\text { "Operative time included as a continu }\end{array}$ & $\begin{array}{l}\text { on; COPD: Chronic Obs } \\
\text { hic attack; CVA: Cerebro } \\
\text { ariable in the regressio }\end{array}$ & $\begin{array}{l}\text { uctive Pulmonary D } \\
\text { ascular accident; W } \\
\text { analysis }\end{array}$ & $\begin{array}{l}\text { CAD: } \\
\text { hite blood ce }\end{array}$ \\
\hline
\end{tabular}




\section{Abstracts of the 2014 Meeting of the Lumbar Spine Research Society}

known life-threatening complication following orthopaedic procedures. However, there is limited data on the incidence, risk factors, and peri-operative outcomes for PE following lumbar spine surgery (LSS).

Methods: The National Surgical Quality Improvement Program (NSQIP) database was utilized to identify patients who underwent elective LSS. Patients with a PE were compared to unaffected patients. Preoperative patient characteristics, lab values, and peri-operative outcomes were assessed. Regression analysis was performed to identify predictors for a postoperative PE with a $95 \%$ confidence interval. SPSS v.20 was utilized for statistical analysis with a p-value of $\leq 0.05$ to denote significance.

Results: A total of 22,610 LSS were identified between 2006-2011, of which $79(0.3 \%)$ demonstrated a postoperative PE. The affected cohort was older, and demonstrated a higher BMI and a greater number of comorbidities. PE patients underwent a greater proportion of non-ambulatory surgeries and incurred a longer operative time than non-PE patients. In addition, affected patients incurred a longer hospitalization, greater readmission rates, and a higher incidence of postoperative deep vein thrombosis and mortality than unaffected patients. Risk factors associated with a postoperative PE included obesity, elevated white blood cell count (WBC), and prolonged operative time. Interestingly, smoking was associated with a reduced risk for a postoperative PE.

Discussion/Conclusion: This study demonstrated that patients with a postoperative PE incurred a longer hospitalization, a higher readmission rate, and greater mortality than unaffected patients. Furthermore, risk factors associated with a postoperative PE included obesity, elevated WBC and a longer operative time. Understanding the incidence and risk factors associated with a PE may assist in the prevention, diagnosis, and treatment of this potentially fatal complication.

Poster 18. Incidence, Predictors and Outcomes of Pneumonia after Lumbar Spine Surgery

Alejandro Marquez-Lara, MD; Sreeharsha V. Nandyala, BA; Steven J. Fineberg, MD; Kern Singh, MD

Rush University Medical Center, Orthopaedic Surgery

Disclosures: A. Marquez-Lara: None. S.V. Nandyala: None. S.J. Fineberg: None. K. Singh: 1; I; Pioneer, Stryker, Zimmer. 4; I; Zimmer, Stryker, Globus. 8; I; Vital 5 LLC. 9; I; Thieme, LWW.

Introduction: Postoperative pneumonia (PNA) is a frequent complication following orthopaedic procedures. However, there is limited data in the published literature regarding the peri-operative risk factors associated with PNA after lumbar spine surgery (LSS).

Methods: Patients who underwent LSS were identified in the National Surgical Quality Improvement Program (NSQIP) database. Patients with postoperative PNA were identified and compared to unaffected patients. Patient characteristics, lab values, and perioperative outcomes were assessed. Regression analysis was performed to identify predictors for postoperative PNA with a 95\% confidence interval. SPSS v. 20 was utilized for statistical analysis with a p-value of $\leq 0.05$ to denote significance.

Results: A total of 22,676 LSS were identified between 2006-2011, of which $85(0.4 \%)$ incurred postoperative PNA. Affected patients were significantly older, and demonstrated a higher BMI and a greater number of comorbidities. The PNA cohort underwent a greater proportion of non-ambulatory surgeries and demonstrated an increased number of blood transfusions and a longer operative time. In addition, affected patients demonstrated a longer hospitalization, greater readmission and reoperation rates, and a higher incidence of postoperative complications. Risk factors associated with postoperative PNA included obesity, chronic obstructive pulmonary disease (COPD), peripheral vascular disease (PVD), and a longer operative time.

\begin{tabular}{|c|c|c|c|}
\hline \multicolumn{4}{|c|}{$\begin{array}{l}\text { Table 1: Comparison of Patient Characteristics with and without } \\
\text { Pneumonia }\end{array}$} \\
\hline Variable & No Pneumonia & Pneumonia & p-value \\
\hline Count (\%) & $22,591(99.6 \%)$ & $85(0.4 \%)$ & \\
\hline Age (years), mean (SD) & $56.62(15.4)$ & $63.79(12.7)$ & $<0.0001$ \\
\hline$\%$ Female & $48.6 \%$ & $34.1 \%$ & 0.008 \\
\hline \multicolumn{4}{|c|}{ Pre-operative Health and Co-morbidities } \\
\hline & $29.97(6.39)$ & $31.48(6.93)$ & 0.03 \\
\hline Diabetes mellitus (\%) & $14.6 \%$ & $24.7 \%$ & 0.009 \\
\hline Smoking & $23.9 \%$ & $23.5 \%$ & 0.94 \\
\hline Alcohol & \multirow{2}{*}{$3.2 \%$} & $3.2 \%$ & 0.99 \\
\hline COPD & & $16.5 \%$ & $<0.0001$ \\
\hline Cardiac $\mathrm{Hx} / \mathrm{CAD}$ & $\begin{array}{l}3.4 \% \\
9.0 \%\end{array}$ & $21.0 \%$ & 0.001 \\
\hline Hypertension & $49.7 \%$ & $97.1 \%$ & 0.001 \\
\hline Peripheral vascular disease & $1.0 \%$ & $6.5 \%$ & $<0.0001$ \\
\hline Dialysis & $0.2 \%$ & $0.0 \%$ & 0.71 \\
\hline $\mathrm{Hx}$ of CVA & $2.2 \%$ & $3.2 \%$ & 0.59 \\
\hline Paralysis & \multirow{2}{*}{$4.8 \%$} & $1.6 \%$ & 0.25 \\
\hline Metastatic cancer & & $0.0 \%$ & 0.72 \\
\hline Open wound/ wound infection & $0.1 \%$ & $1.2 \%$ & 0.53 \\
\hline Steroid Use & $\begin{array}{l}0.6 \% \\
3.0 \%\end{array}$ & $12.9 \%$ & $<0.0001$ \\
\hline \multicolumn{4}{|c|}{$\begin{array}{l}\text { SD: Standard Deviation; COPD: Chronic Obstructive Pulmonary Disease; CAD: Coronary Artery Disease; } \\
\text { CVA: Cerebrovascular accident }\end{array}$} \\
\hline \multicolumn{4}{|c|}{ Comparison of Surgical and 30-day Outcomes Associated with Pneumonia } \\
\hline Ambulatory Surgery (\%) & $19.9 \%$ & $4.7 \%$ & $<0.0001$ \\
\hline Operative time, mean (SD) & \multirow{2}{*}{$\begin{array}{l}146.3(96.1) \\
0.150(0.67)\end{array}$} & $210.5(116.9)$ & $<0.0001$ \\
\hline No. of Blood transfusions, mean (SD) & & $0.722(1.90)$ & $<0.0001$ \\
\hline Length of stay (days), mean (SD) & $2.59(2.96)$ & $9.01(7.45)$ & $<0.0001$ \\
\hline Readmitted (\%) & $5.0 \%$ & $24.3 \%$ & $<0.0001$ \\
\hline Reoperations (\%) & $2.7 \%$ & $9.8 \%$ & 0.005 \\
\hline Unplanned intubation (\%) & $0.2 \%$ & $15.3 \%$ & $<0.0001$ \\
\hline Ventilator $>48$ hours $(\%)$ & $0.1 \%$ & $15.3 \%$ & $<0.0001$ \\
\hline Sepsis (\%) & $0.7 \%$ & $24.7 \%$ & $<0.0001$ \\
\hline Mortality (\%) & $0.1 \%$ & $3.5 \%$ & $<0.0001$ \\
\hline Independen & Predictors for I & umonia & \\
\hline Obesity (BMI > 30) & \begin{tabular}{l|l}
1.84 & \\
\end{tabular} & $1.05 \cdot 3.21$ & 0.03 \\
\hline History of COPD & 2.65 & $1.18 \cdot 5.96$ & 0.02 \\
\hline Peripheral Vascular Disease & 3.94 & $1.24-12.5$ & 0.02 \\
\hline Elevated Creatinine & 2.10 & $1.05 \cdot 4.19$ & 0.04 \\
\hline \begin{tabular}{|l|l|} 
Operative Time* & \\
\end{tabular} & \begin{tabular}{l|l}
1.004 & \\
\end{tabular} & $1.002-1.006$ & $<0.0001$ \\
\hline $\begin{array}{l}\text { SD: Standard Deviation; COPD: Chronic Ot } \\
\text { CVA: Cerebrovaseular aceident } \\
\text {-Operative time included as a continuou }\end{array}$ & tructive Pulmonary & $\begin{array}{l}\text { ease; CAD: Coror } \\
\text { egression }\end{array}$ & ery Disease; \\
\hline
\end{tabular}

Discussion/Conclusion: In this study postoperative PNA was associated with a greater incidence of perioperative complications, and longer hospitalization. In addition, multiple risk factors including obesity COPD, PVD and a longer operative time were associated with an increased risk of postoperative PNA. In light of these findings, further research is warranted to determine if optimization of these perioperative risk factors can mitigate the impact of PNA on hospital resource utilization and postoperative outcomes.

Poster 19. Risk Factors For Extended Length Of Stay Following Elective Posterior Lumbar Fusion

Bryce A. Basques, BS; Michael C. Fu, BS; Rafael A. Buerba, BA; Daniel D. Bohl, MPH; Nicholas S. Golinvaux, BA; Jonathan N. Grauer, MD

Yale School of Medicine, Orthopaedics and Rehabilitation

Disclosures: B.A. Basques: None. M.C. Fu: None. R.A. Buerba: None. D.D. Bohl: None. N.S. Golinvaux: None. J.N. Grauer: 2; I; Stryker. 4; I; Affinergy, Alphatec, Bioventus, Depuy, Harvard Clinical Research Institute, Powered Research, Stryker, Transgenomic.

Introduction: Posterior lumbar spine fusion is a common surgical procedure used to treat lumbar spine pathology. Hospital length of stay (LOS) after posterior lumbar fusion is an important clinical variable and a major determinant of inpatient hospital costs. However, information regarding which factors are associated with increased LOS after posterior lumbar fusion is sparse.

Methods: Patients who underwent elective posterior lumbar fusion from 2005 to 2010 were identified in the American College of Surgeons National Surgical Quality Improvement Program (ACSNSQIP) database using the Current Procedural Terminology billing 


\section{Abstracts of the 2014 Meeting of the Lumbar Spine Research Society}

Table 1: Multivariate analysis for change in length of stay (LOS) associated with each preoperative and intraoperative variable

\begin{tabular}{|c|c|c|c|}
\hline & Change in LOS (days) & $p$ & \\
\hline \multicolumn{4}{|l|}{ Preoperative variables } \\
\hline Aget & +0.34 & $<0.001$ & * \\
\hline Morbid obesity & +1.42 & $<0.001$ & * \\
\hline Male sex & +0.16 & 0.317 & \\
\hline Smoking history & -0.36 & 0.062 & \\
\hline Alcohol history & -0.43 & 0.300 & \\
\hline ASA class $\geq 3$ & +0.58 & 0.001 & * \\
\hline Diabetes & +0.24 & 0.245 & \\
\hline Hypertension & -0.11 & 0.541 & \\
\hline Pulmonary comorbidity & +0.25 & 0.486 & \\
\hline Cardiac comorbidity & +0.01 & 0.967 & \\
\hline \multicolumn{4}{|l|}{ Operative variables } \\
\hline Operative time $\geq 268$ minutes $\$$ & +0.97 & $<0.001$ & * \\
\hline Multilevel procedure & +0.78 & 0.001 & * \\
\hline Intraoperative transfusion & +1.76 & $<0.001$ & $*$ \\
\hline
\end{tabular}

ASA $=$ American Society of Anesthesiologists

$* p<0.05$

$\dagger$ Starting from age 40, brackets defined as $<40,40-49,50-59,60-69,70-79$, and

$\geq 80$. Change in LOS reported per every 10 -year increase in age.

Morbid Obesity defined as body mass index $\geq 40 \mathrm{~kg} / \mathrm{m}^{2}$

$\$ 268$ minutes is the 75 th percentile operative time for this cohort

codes for posterior lumbar arthrodesis. Preoperative and intraoperative variables were extracted for each case and multivariate linear regression was performed to assess the contribution of each variable to LOS.

Results: A total of 1,861 elective posterior lumbar fusion patients were identified. The average age for patients in this cohort was $60.6 \pm$ 13.9 years (mean \pm standard deviation) and average body mass index (BMI) was $30.3 \pm 6.2 \mathrm{~kg} / \mathrm{m} 2.44 .7 \%$ of patients were male. Average LOS was $4.2 \pm 3.3$ days and LOS ranged from 0 to 51 days.

Multivariate analysis (Table 1) identified six independent predictors of increased hospital LOS after lumbar fusion: age ( $p<0.001)$, morbid obesity $(p<0.001)$, ASA class $(p=0.001)$, operative time $\geq$ 75 th percentile or 268 minutes $(\mathrm{p}<0.001)$, multilevel procedure $(\mathrm{p}=$ $0.001)$, and intraoperative transfusion $(\mathrm{p}<0.001)$. Sex, smoking history, alcohol history, diabetes, hypertension, pulmonary comorbidity, and cardiac comorbidity were not significantly associated with length of stay.

Conclusions: Increased age, morbid obesity, ASA class, operative time, number of levels fused, and intraoperative transfusion were all significantly associated with extended LOS following elective posterior lumbar fusion. Patients deemed at-risk based on their preoperative status should be counseled before surgery so they have realistic expectations about their postoperative course.
Poster 20. Comparison of Comorbidity and the Relative Risk Increase for In-Hospital Mortality and Complications Following Lumbar Spine Surgery

Sreeharsha V. Nandyala, BA; Alejandro Marquez-Lara, MD; Hamid Hassanzadeh, MD; Kern Singh, MD

\section{Rush University Medical Center, Orthopaedic Surgery}

Disclosures: S.V. Nandyala: None. A. Marquez-Lara: None. H. Hassanzadeh: None. K. Singh: 1; I; Pioneer, Stryker, Zimmer. 4; I; Zimmer, Stryker, Globus. 8; I; Vital 5 LLC. 9; I; Thieme, LWW.

Introduction: The published literature lacks data evaluating the association between a rising level of comorbidity and the relative risk for postoperative complications and mortality following a lumbar fusion.

Methods: Data from the Nationwide Inpatient Sample was queried from 2002-2011. Patients who underwent an index lumbar fusion were identified and stratified based upon each patient's modified Charlson Comorbidity Index (CCI). Postoperative inpatient mortality, thromboembolism, wound infection, cardiac event, renal failure, and ileus were assessed. The relative risks in each CCI cohort were calculated in reference to the previous respective CCI group in order to investigate the risk increase with a rising comorbidity burden. SPSS v.20 was utilized for statistical analysis with a $95 \%$ confidence interval for relative risk.

Results: A total of 315,597 lumbar arthrodesis procedures were recorded from 2002-2011. The initial relative risk increase for inpatient mortality, pulmonary embolism (PE), wound infection, cardiac events, and renal failure was significant beginning with a CCI score of 2 and compounded for the subsequent CCI cohorts. The risk increase for mortality was most dramatic for patients with a CCI score $>5$. The initial significant increase in relative risk for deep vein thrombosis (DVT) was demonstrated in less comorbid patients with the CCI of 1 , and was consistently significant for the subsequent CCI groups.

Discussion: This analysis of the NIS database demonstrates that the CCI can be utilized to estimate the relative risk increase for in-hospital mortality and complications following lumbar arthrodesis. This study suggests that the risk increase for in-hospital mortality and catastrophic complications initiates in less comorbid patients with a CCI of 1 and 2. Further studies are warranted to investigate the etiologies for this risk increase of complications following lumbar arthrodesis.

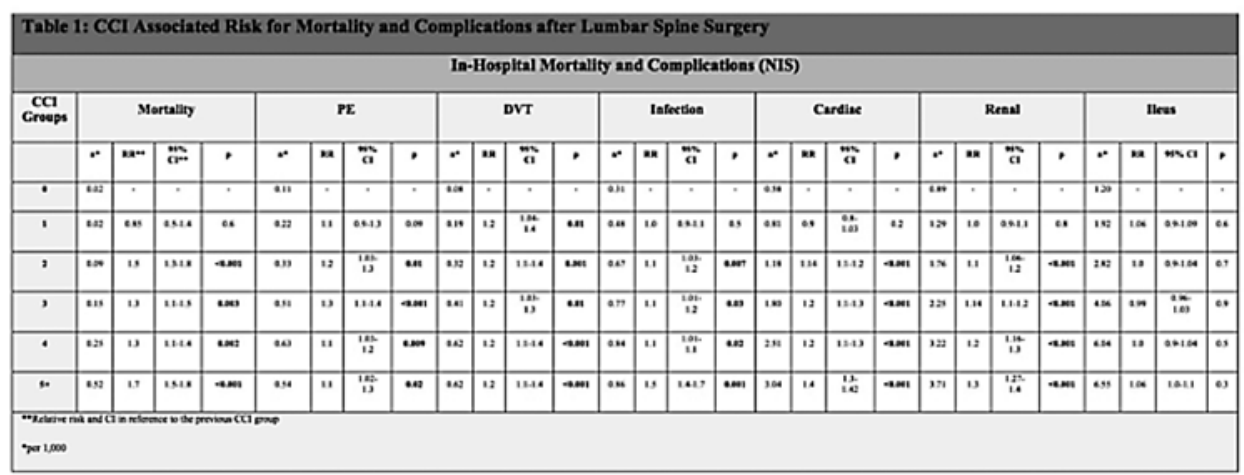




\section{Abstracts of the 2014 Meeting of the Lumbar Spine Research Society}

Poster 21. Sentinel Events in Lumbar Spine Surgery

Alejandro Marquez-Lara, MD; Sreeharsha V. Nandyala, MDs; Hamid Hassanzadeh, MD; Kern Singh, MD

\section{Rush University Medical Center, Orthopaedic Surgery}

Disclosures: A. Marquez-Lara: None. S.V. Nandyala: None. H. Hassanzadeh: None. K. Singh: 1; I; Pioneer, Stryker, Zimmer. 4; I; Zimmer, Stryker, Globus. 8; I; Vital 5 LLC. 9; I; Thieme, LWW.

Introduction: Sentinel events in lumbar spine surgery can have significant medical, social, economic, and legal implications. The incidence and perioperative outcomes associated with these complications have not been well characterized.

Methods: Data from the Nationwide Inpatient Sample was queried from 2002-2011. Patients who underwent lumbar spinal surgery were identified. Sentinel events including bowel or peritoneal injury, vascular injury, nerve injury, retention of foreign objects, and wrong-site surgery were identified. Patient demographics, comorbidities (CCI), length of stay (LOS), total costs, and perioperative outcomes were assessed. The risk for mortality associated with each complication was calculated utilizing a 95\% confidence interval (CI). Statistical analysis was performed with SPSS v.20 and a p-value of $<0.001$ denoted significance.

Results: A total of 543,146 lumbar spine surgeries were recorded from 2002-2011 of which 414 (0.8 per 1,000 cases) incurred sentinel events. Wrong site surgeries were the most common sentinel events with an incidence of 0.3 per 1,000 cases. The incidences for bowel or peritoneal injury, vascular injury, nerve injury, and retention of foreign objects, were $0.06,0.2,0.2$, and 0.1 per 1,000 cases respectively. There were no significant differences in the mean age (55.9

\begin{tabular}{|c|c|c|c|}
\hline Comparison Group & No Complications & Sentinel Events & \multirow[t]{2}{*}{ p-value } \\
\hline Total ( $\mathrm{n}=$ count) & 542,732 & 414 & \\
\hline Age (years) & $55.9( \pm 15.4)$ & $56.0( \pm 14.6)$ & 0.911 \\
\hline $\begin{array}{c}\text { Charlson } \\
\text { Co-morbidity Index }\end{array}$ & $2.58( \pm 1.76)$ & $2.63( \pm 1.81)$ & 0.553 \\
\hline 3+ Levels (\%) & 4.3 & 7.7 & $<0.001$ \\
\hline Teaching Hospitals (\%) & 53.5 & 51.5 & 0.401 \\
\hline LOS (days) & $2.8( \pm 2.3)$ & $5.6( \pm 6.1)$ & $<0.001$ \\
\hline Costs (US dollars) & $15,260( \pm 14,320)$ & $27,655( \pm 25,274)$ & $<0.001$ \\
\hline Mortality (per 1,000 cases) & 0.7 & 14.6 & $<0.001$ \\
\hline $\begin{array}{c}\text { Complications } \\
\text { (per 1,000 cases) } \\
\text { Aspiration } \\
\text { Cardiac Event } \\
\text { DVT } \\
\text { Hematoma } \\
\text { Hemorrhagic Anemia } \\
\text { Neurologic } \\
\text { PE } \\
\text { Re-intubation } \\
\text { UTI } \\
\text { Ileus } \\
\text { SSI }\end{array}$ & $\begin{array}{c}7.3 \\
6.9 \\
1.4 \\
7.6 \\
59.1 \\
6.7 \\
1.4 \\
2.1 \\
14.9 \\
18.4 \\
3.9 \\
\end{array}$ & $\begin{array}{c}36.2 \\
31.4 \\
14.5 \\
74.9 \\
166.7 \\
144.9 \\
9.7 \\
12.1 \\
38.6 \\
108.7 \\
19.3\end{array}$ & $\begin{array}{l}<0.001 \\
<0.001 \\
<0.001 \\
<0.001 \\
<0.001 \\
<0.001 \\
<0.001 \\
<0.001 \\
<0.001 \\
<0.001 \\
<0.001\end{array}$ \\
\hline \multicolumn{4}{|c|}{ Risk of Death with each Sentinel Event } \\
\hline Variable & Relative Risk* & $\begin{array}{c}\mathbf{9 5} \% \text { Confidence } \\
\text { Interval }\end{array}$ & p-value \\
\hline Bowel/Peritoneal Injury & 200.9 & $80.2-503.1$ & $<0.001$ \\
\hline Vascular Injury & 18.4 & 2.6-129.7 & 0.003 \\
\hline Nerve Injury & 6.8 & $0.4-108.0$ & 0.17 \\
\hline Retained Foreign Object & 13.5 & $0.8-213.9$ & 0.06 \\
\hline Wrong-site Surgery & 10.6 & $1.5-74.9$ & 0.02 \\
\hline $\begin{array}{l}\text { All Intraoperative } \\
\text { Complications }\end{array}$ & 22.0 & $9.9-49.1$ & $<0.001$ \\
\hline
\end{tabular}

vs $56.0, \mathrm{p}=0.911)$ or comorbidity burden $(2.58$ vs $2.63, \mathrm{p}=0.553$ ) between the two cohorts. The sentinel event cohort incurred a longer hospitalization, greater costs, and a greater incidence of in-hospital complications and mortality. Patients with a bowel or peritoneal injury, vascular injury, and wrong-site surgery demonstrated a greater risk of mortality relative to unaffected patients.

Conclusions: This NIS analysis demonstrates that sentinel events are associated with a significant increase in hospital resource utilization and worsened postoperative outcomes including death. This study demonstrates the financial and medical burden associated with perioperative complications.

\section{Poster 22. Outcome Of A Resident Spine Surgical Skills Training Program}

Brian P. Calio; John D. Koerner, M.D.; Kris E. Radcliff, M.D.

Jefferson Medical College, Medical College (student)

Disclosures: B.P. Calio: None. J.D. Koerner: None. K.E. Radcliff: None.

Introduction: Resident training is an important topic in the setting of work hour reform. To our knowledge, there are no established curricula for training orthopedic surgery residents on anatomy and common procedures encountered during a spinal surgery rotation. Our hypothesis was that an in-depth technical cadaveric skills training would increase resident confidence and improve operating room safety.

Methods: Residents $(\mathrm{n}=17)$ rotating on the spine service were assembled into teams including a PGY-5, PGY-2, and PGY-1, to perform dissection on cadavers. With attending and fellow supervision, residents performed anterior cervical, posterior cervical, and posterior thoracolumbar surgical exposure, decompression, and laminectomy. Each resident was expected to operate, assist, and identify critical structures. Residents were then queried about their prior knowledge, satisfaction in the operating room, and expectation of safety culture following the training using Likert scale (0-10). Strong agreement (scores > 7) and strong disagreement (scores <4) and correlations were evaluated.

Results: Residents strongly agreed that the team-specific cadaveric training was more helpful than textbook chapters (100\%), sawbones $(94 \%)$, web based (94\%), or simulator based training (94\%). Following the training, the majority of residents strongly agreed with "eager to participate in spine surgery" (94\%), "good understanding of spine anatomy and surgical procedures" $(82 \%)$, and "satisfaction with their current spine training" (82\%). A majority of residents strongly agreed that the training improved feelings of preparation, (52\%), safety (64\%), and ability to prevent intraoperative errors (64\%).

Discussion: This study evaluated a cadaveric based spinal surgical simulation for residents, and compared the effectiveness with traditional teaching methods. Results were overwhelmingly positive, and demonstrate that team based, cadaveric training with adequate attending supervision prior to the onset of a surgical rotation may lead to high resident satisfaction and improve patient safety. This suggests that integration of similar cadaver-based programs into standardized curricula would be met with positive resident feedback.

Conclusion: Cadaveric based resident surgical skills training programs are beneficial and may be more helpful than traditional forms of surgical training from a resident perspective. With work hour restrictions, this method of training may become an important addition to training curricula. 


\section{Abstracts of the 2014 Meeting of the Lumbar Spine Research Society}

\section{Poster 23. The Burden of Clostridium Difficile After Lumbar Spine Surgery}

Javier Guzman, BS; Branko Skovrlj, MD; Young Lu, BA; Motasem Al-Maaieh, MD; Natalia N. Egorova, MPH, PhD; Sheeraz A. Qureshi, MD, MBA

Icahn School of Medicine at Mount Sinai, Department of Orthopaedics

Disclosures: J. Guzman: None. B. Skovrlj: None. Y. Lu: None. M. Al-Maaieh: None. N.N. Egorova: None. S.A. Qureshi: 1; I; Zimmer. 2; I; Medtronic, Stryker Spine. 4; I; Orthofix, Medtronic, Stryker Spine, Zimmer.

Introduction. Hospital-acquired C. difficile infection, resulting in pseudomembranous colitis, confers significant morbidity and mortality. The pathophysiology of this infection is associated with alterations in the physiologic gut flora that can be affected by perioperative antibiotic use. The purpose of this study was to investigate incidence, comorbidities and impact on health care resources associated with $\mathrm{C}$. difficile infection in patients after lumbar spine surgery.

Methods. The National Inpatient Sample was examined from 2002 to 2011. Patients were included for study based on ICD-9-CM procedural codes for lumbar spine surgery and further substratified to degenerative diagnoses. Baseline patient characteristics such as age, insurance type, major comorbidities, costs and mortality rate were determined. Multivariable analyses assessed factors increasing incidence of diagnosis and factors associated with inpatient mortality.

Results. Incidence of $\mathrm{C}$. difficile infection in postoperative lumbar spine surgery patients is $.12 \%$. At baseline, $\mathrm{C}$. difficile patients have an increased incidence of comorbidities such as diabetes with chronic complications, congestive heart failure, coagulopathy and pulmonary circulatory disorders. Lumbar fusion $(\mathrm{P}<.0001)$ and fusion revision $(\mathrm{P}=.001)$ are associated with increased incidence as compared to posterior lumbar decompression. Likewise, Medicaid $(\mathrm{P}<.0001)$

\begin{tabular}{|c|c|c|c|c|}
\hline Risk Factors & $\begin{array}{l}\text { Odds } \\
\text { Ratio }\end{array}$ & $\begin{array}{l}\text { Lower } 95 \% \\
\text { CI }\end{array}$ & $\begin{array}{l}\text { Higher } 95 \% \\
\text { CI }\end{array}$ & P-value \\
\hline Patient age & 1.02 & 1.01 & 1.04 & .0005 \\
\hline Female & .98 & .78 & 1.24 & .871 \\
\hline Elixshauser Index & 1.17 & 1.15 & 1.19 & $<.0001$ \\
\hline \multicolumn{5}{|c|}{ Bed Size: Reference Large Hospital } \\
\hline Medium & .64 & .47 & .88 & .463 \\
\hline Small & .54 & .36 & .79 & .046 \\
\hline \multicolumn{5}{|c|}{ Region: Reference Northeast Region } \\
\hline Midwest & .88 & .61 & 1.28 & .514 \\
\hline South & .62 & .44 & .87 & .006 \\
\hline West & .82 & .57 & 1.18 & .999 \\
\hline Urban vs. Rural & 4.12 & 1.52 & 11.12 & .0005 \\
\hline $\begin{array}{l}\text { Academic vs. } \\
\text { Non-Teaching }\end{array}$ & 1.04 & .81 & 1.35 & .743 \\
\hline \multicolumn{5}{|c|}{ Insurance: Reference Private } \\
\hline Medicare & .98 & .69 & 1.40 & $<.0001$ \\
\hline Medicaid & 1.73 & 1.12 & 2.68 & $<.0001$ \\
\hline Uninsured & 1.58 & .48 & 5.24 & $<.0001$ \\
\hline Other & .93 & .51 & 1.68 & $<.0001$ \\
\hline Missing & $<.001$ & $<.001$ & $<.001$ & $<.0001$ \\
\hline \multicolumn{5}{|c|}{ Procedure: Reference Lumbar Decompression } \\
\hline Lumbar Fusion & 1.97 & 1.47 & 2.64 & $<.0001$ \\
\hline Lumbar Fusion Revision & 2.10 & 1.35 & 3.23 & .001 \\
\hline
\end{tabular}

and those uninsured $(\mathrm{P}<.0001)$ have an increased incidence when compared to private insurance. Small hospitals $(\mathrm{P}=.046)$ are associated with decreased likelihood of infection when compared to large hospitals. C. difficile results in extended length of stay $(\mathrm{P}<.0001)$ and higher median costs $(\mathrm{P}<.0001)$. A total of $4.5 \%$ of patients with $\mathrm{C}$. difficile infection died versus $.11 \%$ of patients without $\mathrm{C}$. difficile $(\mathrm{P}$ $<.0001)$. Multivariable analysis for inpatient mortality indicates that C. difficile greatly increases mortality, 7.66 odds ratio $(\mathrm{P}<.0001)$.

Discussion. C. difficile carries a 40.9 fold increase in mortality and costs approximately $\$ 7,143,058$ per year to manage_a significant impact on healthcare resources. Novel and difficult to treat antibiotic resistant strains may be reduced if proper antibiotic administration is practiced in this population.

Conclusion. C. difficile infection profoundly increases in-hospital mortality, length of stay, costs and has a significant impact on hospital resources in the lumbar spine surgery population.

Poster 24. Kinematic Analysis of Diseased and Adjacent Segments in Degenerative Lumbar Spondylolisthesis

Kevin H. Phan; Michael D. Daubs, MD; Asher I. Kupperman, MD; Trevor P. Scott, MD; Jeffrey C. Wang, MD

\section{UCLA, Orthopaedic Surgery}

Disclosures: K.H. Phan: None. M.D. Daubs: 1; I; Depuy-Synthes. 4; I; Depuy-Synthes. 7; I; Stryker. A.I. Kupperman: None. T.P. Scott: None. J.C. Wang: 1; I; Biomet, Stryker, Medtronics, Depuy-Synthes, Amedica, Osprey, Aesculap, Seaspine. 6; I; Fziomed, Promethean Spine, Paradigm Spine, Benevenue, NexGen, Pioneer, Amedica, Vertiflex, Electrocore, Surgitech, Axiomed, VG Innovations, Corespine, Expanding Orthopaedics, Syndicom, Osprey, Bone Biologics, Curative Biosciences, Pearldiver.

Introduction: Degenerative spondylolisthesis (DS) is a common pathological condition which can lead to lumbar instability and significant spine symptoms. How this disease process affects adjacent lumbar motion segments has not been studied. The purpose of this study was to characterize the motion characteristics of lumbar DS at the diseased and adjacent levels in patients with low-grade, singlelevel lumbar DS using kinetic MRI (kMRI).

Methods: The study included 112 patients with a diagnosis of lowgrade (Grade 1 or 2), single-level lumbar DS with mean age 50 years (22-85 years): 22 with L3-4 spondylolisthesis (mean 53 years, 32-68 years), 55 with L4-5 spondylolisthesis (mean 51 years, 22-85 years), and 35 with L5-S1 spondylolisthesis (45 years, 24-72 years) and 296 control patients without spondylolisthesis. Angular motion (AM) and translational motion (TM) were measured using kMRI images and MR Analyzer software. Level of slip was graded according to Meyerding's classification system and disc degeneration according to Pfirrmann. TM at a motion segment $>4 \mathrm{~mm}$ was categorized as instability.

Results: Lumbar hypomobility was present in all spondylolisthesis patients, however slip at L3-4 resulted in the largest decrease in lumbar range of motion. Instability was similar at the spondylolisthesis

level at all levels: L3-4 (36\%), L5-S1 (31\%), and L4-5 (30\%) $(\mathrm{p}>0.05)$. Patients with a stable spondylolisthesis generally had decreased AM at all lumbar levels. Patients with unstable slip at L3-4 and L4-5 exhibited compensatory motion at adjacent segments ( $>0.05)$. TM in patients with DS was consistently increased.

Discussion: AM is decreased at the diseased level with L3-4 or L5-S1 DS but increased with L4-5 DS; TM was increased at the diseased level in all patients. Patients with L4-5 spondylolisthesis had the highest incidence of segmental instability at adjacent segments.

Conclusions: About a third of patients with single-level DS at L3-4, L4-5, or L5-S1 will have instability at that level. There is a tendency for compensatory motion at adjacent levels in patients with unstable spondylolisthesis at L3-4 and L4-5 but not L5-S1. 


\section{Abstracts of the 2014 Meeting of the Lumbar Spine Research Society}

Poster 25. Utility of Intraoperative Monitoring in Thoracolumbar Kyphoplasty

Don Moore

Cleveland Clinic, Neurological Institute

Disclosures: D. Moore: 6; I; Medtronic.

Study Design: Restrospective

Objective. The goal of the study was to determine the necessity of intraoperative neuromonitoring (IOM) during thoracic and upper lumbar kyphoplasty (KP) in detecting potential neurological deterioration.

Summary of Background Data. IOM was used to detect any neurological deficit from mechanical compromise of the canal. There is a paucity of data supporting the use of intraoperative monitoring during KP.

Methods. A retrospective review of a single surgeon in a nonacademic setting was performed from August 1, 2007 through July 31,2013 . IOM was used for all thoracic kyphoplasties and selectively whenever risks were present for potential neurological complications, such as in presence of minor burst fracture with canal compromise, asymptomatic cervical stenosis or recent administration of long lasting anticoagulants. Postoperative neurological complication was assessed along with the level of the surgery, unilateral, bilateral as well as extrapedicular or transpedicular approaches.

Results. There were a total of 158 consecutive cases in 134 patients who underwent kyphoplasty ranging from T5 to L2. There were 93 female and 41 male patients. The average age for females was 79 and for males was 78. 110 of the 158 cases were at the level of the thoracolumbar junction from T-11 to L2. 3 out of 108 transpedicular cases were unilateral and 1 out of 58 extrapedicular cases was bilateral. There were 24 minor burst fractures. 2 patients underwent simultaneous decompression laminectomy due to presence of neurological deficit. Complication included one aborted, one right pneumothorax, one postoperative CVA, and one postoperative ruptured anterior abdominal aortic aneurysm with mortality. 8 out of 34 cases, in which IOM was not used, were extrapedicular. Only 1 case was in thoracic spine in a patient who previously had an adjacent upper level treated with kyphoplasty.

Conclusion. There was no postoperative neurological deterioration in any of the patients. No IOM changes were detected. With an average cost of $\$ 1,500$ to $\$ 2,000$ for IOM, the cost saving would have been $\$ 186,000-\$ 248,000$ for 124 cases in which IOM was used. This limited review suggests that no intraoperative neurological compromise occurred during thoracic and thoracolumbar kyphoplasty. Nevertheless, it cannot be concluded that IOM is not necessary in current litigious environment and with patients with multiple comorbidities.

\section{Poster 26. The Prevalence and Impact of Low Back and Leg Pain} Among Aging Canadians: A Cross-Sectional Survey

\section{Y. Raja Rampersaud; Kala Sundararajan, MSc; Susan Eng, LL.B}

University Health Network, Toronto Western Hospital, University of Toronto, Orthopaedics

Disclosures: Y. Rampersaud: 4; I; Medtronic. K. Sundararajan: None. S. Eng: None.

Introduction: The objectives of this study were: 1) To characterize the prevalence of low back and leg pain in the aging population, 2) To determine the self-reported impact of lumbar symptoms on quality of life (QOL) and 3) to assess the attitudes of these individuals towards surgical management.

Methods: A voluntary, cross-sectional online survey regarding lumbar symptoms, symptom severity and treatment considerations was made available to members of Canadian Association of Retired Persons (CARP) in 2012.

Results: Out of 3757 total responses, 2781 complete questionnaires were analyzed. Most respondents were men (59\%) and were from Ontario (59\%). From the entire sample, over half of all respondents $(60 \%)$ reported some level of lumbar spinal symptoms: back pain only (23\%); leg pain only (9\%); both back and leg (likely lumbar spinal stenosis (LSS) -28\%). Seventy-two percent of those with spine symptoms saw a physician. Spinal symptoms affected their quality of life "quite a bit" or "a great deal" in $38 \%$ of those with both back and leg symptoms and $11 \%$ in those with back pain only. About a quarter of respondents with leg and back symptoms (24.4\%) reported that they had been diagnosed with a "pinched nerve" (i.e., probable LSS). Of respondents who were diagnosed with a "pinched nerve", the majority $(56.4 \%)$ reported that they were told that "nothing could be done" for treatment, and $74.8 \%$ reported that they would not consider surgery.

Conclusions: Back and leg symptoms are highly prevalent with a significant impact on the quality of life in the aging population. Approximately half of symptomatic individuals may be suffering from LSS, but the majority are unaware of or unwilling to consider the value of surgery for their symptoms. It is likely that LSS in being undertreated in the aging population; however, this assertion requires further investigation.

Poster 27. Orthopaedic Surgical AdVerse Event Severity (OrthoSAVES) System: Identifying opportunities for improved patient safety and resource utilization

Y. Raja Rampersaud; Christian Veillette, MD, FRCSC, MSc; Rajiv Gandhi, MS, MD, FRCSC; Diana Adams, MN, RN(EC), CNCC(C); Natasha Briggs, RN(EC), MN, ACNP, ONC(C); J. Roderick Davey, MD, FRCSC; Michael Fehlings, MD, FRCSC, PhD; Johnny Lau, MD, FRCSC; Stephen Lewis, MD, FRCSC, MSc; Rosalie Magtoto, $\mathrm{RN}, \mathrm{BScN}$; K. Wayne Marshall, MD, FRCSC, PhD; Eric Massicotte, MD, FRCSC; Darrell Ogilvie-Harris, MD, FRCSC; Angela Sarro, MN, RN, CNN; Khalid Syed, MD, FRCSC; Nizar Mohamed, MD, $\mathrm{ScD}, \operatorname{FRCS}(\mathrm{C})$

University Health Network, Toronto Western Hospital, University of Toronto, Orthopaedics

Disclosures: Y. Rampersaud: 4; I; Medtronic. C. Veillette: None. R. Gandhi: 4; I; Eli Lilly. 7; I; Smith and Nephew and Biomet. D. Adams: None. N. Briggs: None. J. Davey: 1; I; Biomet. 2; I; Biomet. 4; I; Biomet. M. Fehlings: None. J. Lau: None. S. Lewis: None. R. Magtoto: None. K. Marshall: None. E. Massicotte: None. D. OgilvieHarris: None. A. Sarro: None. K. Syed: None. N. Mohamed: 2; I; Smith and Nephew.

Introduction: The main objectives of this project were to identify factors predicting adverse events (AEs) in orthopaedic and spine surgery, and to identify the clinical and resource impacts of AEs.

Methods: Comprehensive data were prospectively collected on 3453 consecutive inpatient orthopedic and spine surgeries at Toronto Western Hospital between January 2011 and December 2012. Measured outcome variables included number, type and severity of adverse events according to the validated adverse event severity grading system, as well as length of stay (LOS) and patient-level cost. Odds of having an adverse event was modeled with multivariable logistic regression. Length of stay and cost were modeled as outcomes of multiple linear regression.

Results: Twenty-nine percent of all inpatients developed at least one AE; $7 \%$ had more than one. The majority of patients who had adverse events had low severity AEs (grade I-II). The most common post-operative AEs were urinary tract infections (UTIs), delirium, and 


\section{Abstracts of the 2014 Meeting of the Lumbar Spine Research Society}

urinary retention. The odds of having an $\mathrm{AE}$ were higher in patients over age 65 with worse pre-operative ASA status and longer operating times; gender, obesity and diagnosis were not significant predictors. Dependent on anatomical site, AEs accounted for increases in LOS of 1.5 to 7.6 days and cost of $\$ 1800$ to $\$ 18700$. Adverse events were associated with the highest increases in LOS and cost in spine patients: having an AE predicted LOS 7.6 days longer and cost $\$ 18689$ higher in this subgroup. As UTIs were found to account for a large proportion of AEs, a protocol designed to lower the UTI rate in the spine population was implemented and UTI incidence fell from $5 \%$ to $2 \%$.

Conclusions: Adverse events are associated with significantly higher LOS and cost in orthopaedic surgery - especially in spine surgery. Reduction and prevention of adverse events may lead to significant savings in hospital resources.

Poster 28. Smoking is Common but Does Not Increase the Risk of Perioperative Complications Following Elective Lumbar Spine Surgery: A Study of 11,586 Patients in The ACS NSQIP Database

Nicholas S. Golinvaux, BA; Bryce A. Basques, BS; Daniel D. Bohl, MPH; Jonathan N. Grauer, MD

\section{Yale University School of Medicine, Department of Orthopaedics}

Disclosures: N.S. Golinvaux: None. B.A. Basques: None. D.D. Bohl: None. J.N. Grauer: 2; I; Stryker. 4; I; Affinergy, Alphatec, Bioventus, Depuy, Harvard Clinical Reserach Institute, Powered Research, Stryker, Transgenomic.

Introduction: Complications associated with smoking are well documented. These effects are known for general health and cardiovascular disease. In the realm of spinal fusions, smoking is also known to impede bone healing. Based on these established risks, many spine surgeons advise patients to stop smoking prior to elective surgery. However, this is not done by all spine surgeons, and patient compliance remains an issue.

True smoking prevalence in the modern-day elective spine population has not been well documented. Furthermore, the clinical impact of smoking on perioperative complications has not been well established.

Methods: Based on current procedural terminology (CPT) coding, all elective lumbar discectomy and decompressions and/or fusions logged in the American College of Surgeons National Surgical Quality Improvement Program (ACS NSQIP) database between 2010 and 2012 were identified. This database includes hospital and 30-day follow-up data.

Smoking status was identified for each patient. Bivariate and multivariate regressions were performed to assess for association with perioperative complications, hospital length of stay (LOS), and 30-day hospital readmission. A Bonferroni adjustment for significance level in the setting of multiple comparisons was applied, such that a p-value $<0.002$ was considered significant.

Results: A total of 11,586 patients were identified. 6,590 patients underwent decompressions alone, and 4,996 underwent fusion with or without decompression. The average patient age was $57.4 \pm 15.3$ years (mean \pm standard deviation) and $50.7 \%$ were male. Smoking rates were $20.8 \%$ for those undergoing decompression alone and $20.2 \%$ for fusion. Smoking status was shown not to significantly affect the rate of any of the 23 adverse events evaluated. LOS and hospital readmission were also not affected.

Discussion: We were surprised to find that approximately one fifth of patients undergoing elective spine surgery are smokers. These numbers are actually higher than the national average in the United States. We were further surprised that smoking status was not associated with perioperative complications, LOS, or hospital readmission.

Conclusion: Although smoking has known adverse effects on general health, as well as fusion success, smoking was not found to be associated with perioperative complications in elective lumbar spine surgery patients in this large, national database study.

\section{Poster 29. A Literature Review of Spine Surgery Outcomes in} Patients with Parkinson's Disease

Branko Skovrlj; Christopher Sarkiss; Javier Guzman; Samuel K. Cho; John Caridi

\section{Icahn School of Medicine at Mount Sinai, Neurosurgery}

Disclosures: B. Skovrlj: None. C. Sarkiss: None. J. Guzman: None. S.K. Cho: 4; I; Stryker. J. Caridi: 4; I; Stryker, Zimmer, DePuy.

Introduction: Degenerative spondylosis, thoracolumbar scoliosis and cervical deformity are increasingly recognized entities in patients with Parkinson's disease. Surgical treatment with spinal decompression and/or fusion can be complicated due to poor bone quality and severe muscular dysfunction in this patient population. There is limited information in the literature regarding outcomes of spine surgery patients with Parkinson's disease.

Methods: We performed a literature review using the PubMed and Google Scholar search engines investigating "Parkinson's disease and spinal surgery" covering the period from 2000-2013. Only articles in English journals or published with English language translations were included. Level of evidence of the selected articles was assessed.

Results: A total of 95 patients with Parkinson's disease who underwent spinal surgery were reviewed. The average patient age was 69 with a male to female ratio of 3:4. Average follow up was 40 months (range $14.2-66.8$ ). 46 of 73 patients $(63 \%)$ were judged to have satisfactory outcomes. Poor outcomes were noted in the remaining $37 \%$ and included but were not limited to pseudoarthrosis, hardware failure/pullout, persistent kyphosis or sagittal imbalance, and no improvement or worsening in their postoperative visual ana$\log$ pain scale. Out of this subset of unsatisfactory outcomes, 29 of 65 patients $(45 \%)$ required revisions. For patients undergoing fusion procedures, 55 of $78(71 \%)$ had successful fusion following the index procedure. Complications following index surgery were reported in

\begin{tabular}{|c|c|c|c|c|c|c|c|c|c|}
\hline Author & Location & $\begin{array}{c}\text { Number } \\
\text { of } \\
\text { Patients }\end{array}$ & $\begin{array}{c}\text { Average } \\
\text { Age } \\
\text { (years) }\end{array}$ & $\begin{array}{l}\text { Gender } \\
\text { M:F }\end{array}$ & $\begin{array}{l}\text { Follow-up } \\
\text { Period } \\
\text { (months) }\end{array}$ & $\begin{array}{l}\text { Complication } \\
\text { Rate }\end{array}$ & $\begin{array}{l}\text { Fusion } \\
\text { Rate }\end{array}$ & $\begin{array}{l}\text { Satisfactory } \\
\text { Outcome }\end{array}$ & $\begin{array}{c}\text { Revision } \\
\text { Rate }\end{array}$ \\
\hline $\begin{array}{l}\text { Moon et } \\
\text { al. }\end{array}$ & Lumbar & 20 & 65.2 & $8: 12$ & 56.7 & $n / a$ & $\begin{array}{l}15 / 20 \\
(75 \%)\end{array}$ & $\begin{array}{l}1 / 20 \\
(5 \%)\end{array}$ & $\mathrm{n} / \mathrm{a}$ \\
\hline $\begin{array}{l}\text { Babat et } \\
\text { al. }\end{array}$ & $\begin{array}{l}\text { Cervival, } \\
\text { Thoracic, } \\
\text { Lumbar }\end{array}$ & 14 & 71.3 & $3: 11$ & 66.8 & $\begin{array}{l}10 / 14 \\
(71 \%)\end{array}$ & $\begin{array}{c}3 / 7 \\
(43 \%)\end{array}$ & $n / a$ & $\begin{array}{l}12 / 14 \\
(86 \%)\end{array}$ \\
\hline $\begin{array}{l}\text { Koller et } \\
\text { al. }\end{array}$ & $\begin{array}{c}\text { Thoracic, } \\
\text { Lumbar, } \\
\text { Sacral }\end{array}$ & 23 & 66.3 & $15: 8$ & 14.2 & $\begin{array}{c}12 / 23 \\
(52.2 \%)\end{array}$ & $\begin{array}{l}11 / 18 \\
(61 \%)\end{array}$ & $\begin{array}{l}14 / 18 \\
(78 \%)\end{array}$ & $\begin{array}{l}6 / 18 \\
(33 \%)\end{array}$ \\
\hline $\begin{array}{l}\text { Wadia et } \\
\text { al. }\end{array}$ & $\begin{array}{l}\text { Thoracic, } \\
\text { Lumbar, } \\
\text { Sacral }\end{array}$ & 2 & 74 & $2: 0$ & 48 & $\begin{array}{c}2 / 2 \\
(100 \%)\end{array}$ & $\begin{array}{l}0 / 2 \\
(0 \%)\end{array}$ & $\begin{array}{c}1 / 2 \\
(50 \%)\end{array}$ & $\begin{array}{c}1 / 2 \\
(50 \%)\end{array}$ \\
\hline $\begin{array}{l}\text { Bourghli } \\
\text { et al. }\end{array}$ & $\begin{array}{l}\text { Thoracic, } \\
\text { Lumbar, } \\
\text { Sacral }\end{array}$ & 12 & 68 & $2: 10$ & 32.8 & $\begin{array}{l}6 / 12 \\
(50 \%)\end{array}$ & $\begin{array}{l}9 / 12 \\
(75 \%)\end{array}$ & $\begin{array}{l}11 / 12 \\
(92 \%)\end{array}$ & $\begin{array}{l}6 / 12 \\
(50 \%)\end{array}$ \\
\hline $\begin{array}{l}\text { Kaspar } \\
\text { et al. }\end{array}$ & $\begin{array}{l}\text { Cervical, } \\
\text { Thoracic, } \\
\text { Lumbar }\end{array}$ & 24 & 69 & $n / a$ & 19 & $n / a$ & $\begin{array}{l}17 / 19 \\
(89 \%)\end{array}$ & $\begin{array}{l}19 / 21 \\
(90 \%)\end{array}$ & $\begin{array}{l}4 / 19 \\
(21 \%)\end{array}$ \\
\hline
\end{tabular}




\section{Abstracts of the 2014 Meeting of the Lumbar Spine Research Society}

30 of 51(59\%) of patients. In patients who underwent decompression surgery only, 6 of $6(100 \%)$ worsened post-operatively necessitating a revision multilevel instrumented fusion.

Conclusions: Patients with Parkinson's disease who undergo spinal surgery appear to have high complication and revision rates with poor outcomes and low fusion rates. It remains unclear whether Parkinson's patients benefit from spinal surgery for spinal cord decompression, spinal stabilization and correction of spinal deformities. Further prospective studies are warranted to investigate surgical outcomes in this subset of patients.

Poster 30. Posterolateral Lumbar Fusion with and without Posterior Interbody Fusion for L4-5 Degenerative Spondylolisthesis: A Comparative Value Analysis

Michael Gottschalk; Kyle Sweeney, MD; Scott Boden, MD; John Heller, MD; John Rhee, MD; Tim Yoon, MD

\section{Emory University, Orthopaedics}

Disclosures: M. Gottschalk: None. K. Sweeney: None. S. Boden: 1; I; Medtronic for DBM. 9; I; Saunders for Seminars in Spine Surgery. J. Heller: 1; I; Medtronic for Laminoplasty Plates. 6; I; Medtronic Stock. 7; I; Emory Spine Omega Grant Fellowship. J. Rhee: 1; I; Biomet Spine. 4; I; Biomet Spine. 6; I; Phygen Alphatec. 9; I; Lippincott. T. Yoon: 1; I; Stryker spine. 4; I; Stryker, Meditech. 6; I; Phygen, Alphatec, Meditech, Medyssey.

Introduction: Scant data exists comparing posterolateral lumbar fusion (PLF) with and without posterior interbody fusion (PIF) for degenerative lumbar disorders[1]. Many lumbar surgical advances have been made over the past several decades, yet there is a paucity of strong evidence-based validation, let alone comparative value analyses [2]. Despite the quality of available data, current and previous trends in lumbar spine surgery continue to demonstrate a rise in the number of combined PLF+PIF for routine degenerative diagnoses[3-5]. The objective of this study was to compare the clinical, radiographic, and cost differences of PLF versus PLF+PIF in the setting of L4-5 degenerative spondylolisthesis (DS).

Methods: Patients undergoing single level fusion for L4-5 DS performed at our institution from 2004-2012 were identified. Exclusion criteria included multilevel fusion, spinal stenosis requiring decompression at or above L2-3, previous L4-5 spinal fusion, and use of minimally invasive surgery. Radiographic fusion status, epidemiological, surgical, functional outcome, and cost data were recorded. An independent musculoskeletal radiologist interpreted CT scans for fusion status. Average follow up was 572 and 494 days in the PLF and PLF+PIF groups, respectively.

Results: 240 patients were identified: 88 with PLF and 152 with $\mathrm{PLF}+\mathrm{PIF}$. No statistical differences were noted in ODI, SF-36, fusion rates, or charges/cost. Power analysis demonstrated the ability to detect a difference of $8.76,5.25$, and $\$ 3,471.87 / \$ 1863.85$ for ODI, SF-36, and hospital charges/costs, respectively. Although not statistically significant, the PLF+PIF group had increased hospital charges/ costs, $\$ 1,758.70 / \$ 907.90$.

Discussion: Surgical management of DS is well accepted but may be costly[6-9]. However, the comparative value of circumferential fusion remains to be evaluated[1, 10-15]. Because clinical differences between surgical interventions for DS can take longer to become evident, our study is in the process of collecting current data[13]. Cost analysis is also controversial, as proponents for PLF+PIF could argue that $\$ 1758.70$ is a small percentage of overall costs, while PLF supporters would state that in a grander scheme that difference equates to substantial costs savings.

Conclusion: Given the absence of difference in fusion status and outcomes between the two groups, surgeons should be judicious in choosing their surgical techniques.

Poster 31. Lumbar Fusion versus Non-operative Management for Treatment of Discogenic Low Back Pain: A Systematic Review and Meta-analysis of Randomized Controlled Trials

Mohamad Bydon; Rafael De la Garza-Ramos, M.D.; Mohamed Macki, B.S.; Abdul Baker, M.D.; Daniel M. Sciubba, M.D.; Timothy F. Witham., M.D.; Jean-Paul Wolinksy, M.D.; Ziya L. Gokaslan, M.D.; Ali Bydon

Johns Hopkins University School of Medicine, Neurosurgery

Disclosures: M. Bydon: 4; I; MedImmune, LLC. 7; I; DePuy. R. De la Garza-Ramos: None. M. Macki: None. A. Baker: None. D.M. Sciubba: 4; I; DePuy, Medtronic, Nuvasiv, Globus. 7; I; DePuy. T.F. Witham.: 7; I; Eli Lily and Company. J. Wolinksy: None. Z.L. Gokaslan: 6; I; Spinal Kinetics, US Spine. 7; I; DePuy, AO Spine North America, Medtronic, NREF, Integra Life Sciences, K2M. A. Bydon: 4; I; MedImmune, LLC. 7; I; DePuy.

Introduction: Discogenic low back pain is a common and sometimes disabling condition. When the condition becomes chronic and intractable, spinal fusion may play a role. The purpose of this study is to evaluate the current evidence comparing lumbar fusion to non-operative management for the treatment of chronic discogenic low back pain.

Methods: A systematic review of the literature was conducted using the PubMed and CENTRAL databases. We included RCTs that compared lumbar fusion to non-operative management for the treatment of adult patients with chronic discogenic low back pain. A meta-analysis was conducted to assess the improvement in back pain based on the ODI (Oswestry Disability Index).

Results: Five RCTs met our inclusion criteria. A total of 707 patients were divided into lumbar fusion $(\mathrm{n}=523)$ and conservative management $(n=134)$. The pooled mean difference in ODI (final ODI minus initial ODI) between the non-operative and lumbar fusion groups across all studies was -7.39 points [95\% CI: $-20.26,5.47]$ in favor of lumbar fusion, but this difference was not statistically significant $(\mathrm{p}=0.26)$.

Discussion: Although inclusion / exclusion criteria were relatively similar across studies, surgical techniques and conservative management protocols varied. Despite the significant improvement in ODI

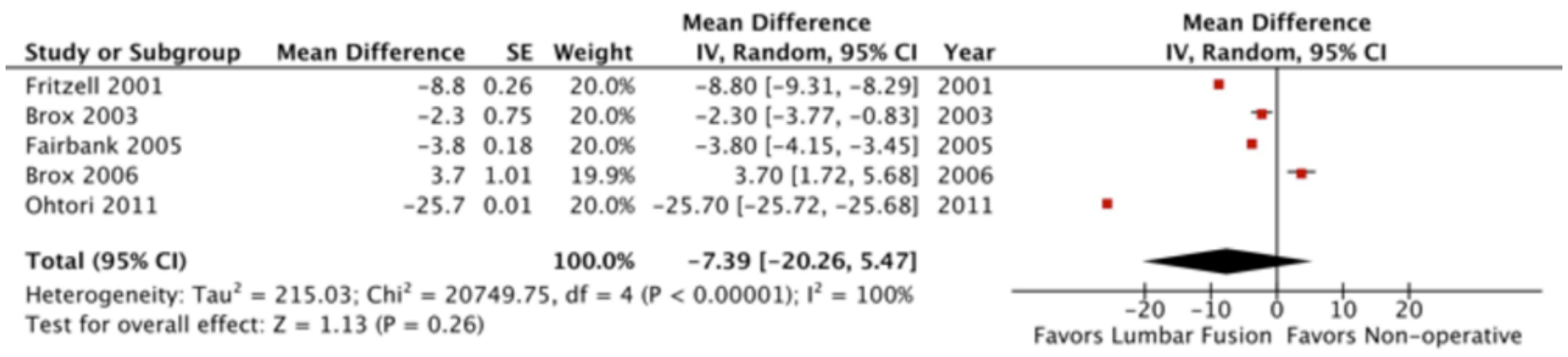




\section{Abstracts of the 2014 Meeting of the Lumbar Spine Research Society}

in the lumbar fusion groups in three studies, pooled data revealed no significant difference when compared to the non-operative group.

Conclusions: Patients receiving a lumbar fusion improved on average 7.39 points in the ODI in favor of lumbar fusion, but it is unclear that this change in ODI would lead to a clinically significant difference. Prospective randomized trials comparing a specific surgical technique versus a structured physical therapy program may improve evidence quality. Until then, either operative intervention via lumbar fusion or non-operative management and physical therapy remain two acceptable treatment methods for intractable low back pain.

Poster 32. Patients with Degenerative Disc Disease have The Highest Rates of Reoperation after Lumbar Fusion: An Analysis of 790 Patients Based on Preoperative Diagnosis

Mohamad Bydon; Rafael De la Garza-Ramos, M.D.; Risheng Xu, Ph.D; Mohamed Macki, B.S.; Nicholas B. Abt, B.S.; Daniel M. Sciubba, M.D.; Jean-Paul Wolinksy, M.D.; Ali Bydon, M.D.; Ziya L. Gokaslan, M.D.; Timothy F. Witham, M.D.

Johns Hopkins University School of Medicine, Neurosurgery

Disclosures: M. Bydon: 4; I; MedImmune,LLC. 7; I; DePuy. R. De la Garza-Ramos: None. R. Xu: None. M. Macki: None. N.B. Abt: None. D.M. Sciubba: 4; I; DePuy, Medtronic, Nuvasiv, Globus. 7; I; DePuy. J. Wolinksy: None. A. Bydon: 4; I; MedImmune, LLC. 7; I; DePuy. Z.L. Gokaslan: 6; I; Spinal Kinetics, US Spine. 7; I; DePuy, AO Spine North America, Medtronic, NREF, Integra Life Sciences, K2M. T.F. Witham: 7; I; Eli Lily and Company.

Introduction: Although outcomes after lumbar fusions are thought to be influenced by pre-operative diagnosis, the extent of this influence has not been well documented. The purpose of this study is to compare outcomes after lumbar fusion based on pre-operative diagnosis.

Methods: Patient records were utilized to identify patients with degenerative spine disease who underwent posterolateral instrumented fusion of the lumbar spine over a 20-year period at our institution. Patients were stratified into three cohorts based on pre-operative diagnosis: spinal stenosis, spondylolisthesis and degenerative disc disease (DDD). Our main outcome variables were symptom resolution at last follow-up, re-operation for adjacent segment disease (ASD) and overall re-operation rates.

Results: A total of 790 patients were identified, with 487 (61.6\%) patients with spinal stenosis, $178(22.5 \%)$ with spondylolisthesis and $125(15.8 \%)$ with DDD. Kaplan-Meier analyses revealed that a significantly higher number of patients with DDD would re-develop back pain over time when compared to patients with spinal stenosis only $(P=0.002)$. Re-operation was also significantly higher in the DDD group over time $(P=0.005)$. Additionally, an incidence rate ratio (IRR) analysis revealed that patients with DDD re-developed back pain 1.7 times faster than patients with spinal stenosis $(\mathrm{P}=0.003)$.

Discussion: The explanation for the worse outcomes after lumbar fusion for patients with DDD may be attributed to a more multifactorial pathology when compared to spinal stenosis or spondylolisthesis. On the other hand, patients with spinal stenosis and spondylolisthesis usually have a more obvious or "direct" cause of their symptoms (i.e. herniated disc, stenosis, vertebral slippage) and hence wide decompression and stabilization is beneficial.

Conclusion: Few studies to date have specifically investigated the influence of pre-operative diagnosis on lumbar fusion outcomes. The present study revealed that patients with DDD who undergo lumbar fusion re-develop back pain at faster and higher rates when compared to patients with spinal stenosis, and also experience higher re-operation rates.
Poster 33. Age is Not an Independent Risk Factor in Postoperative Morbidity after Lumbar Fusion: An Analysis of 1,352 Patients Stratified by Decade Cohorts

Mohamad Bydon; Nicholas B. Abt, B.S.; Israel O. Olorundare, M.B.B.S., M.P.H.; Rafael De la Garza-Ramos, M.D.; Mohamed Macki, B.S.; Kelly McGovern; Daniel M. Sciubba, M.D.; Jean-Paul Wolinksy, M.D.; Timothy F. Witham, M.D.; Ziya L. Gokaslan, M.D.; Ali Bydon, M.D.

\section{Johns Hopkins University School of Medicine, Neurosurgery}

Disclosures: M. Bydon: 4; I; MedImmune, LLC. 7; I; DePuy. N.B. Abt: None. I.O. Olorundare: None. R. De la Garza-Ramos: None. M. Macki: None. K. McGovern: None. D.M. Sciubba: 4; I; DePuy, Medtronic, Nuvasiv, Globus. 7; I; DePuy. J. Wolinksy: None. T.F. Witham: 7; I; Eli Lily and Company. Z.L. Gokaslan: 6; I; Spinal Kinetics, US Spine. 7; I; DePuy, AO Spine North America, Medtronic, NREF, Integra Life Sciences, K2M. A. Bydon: 4; I; MedImmune, LLC. 7; I; DePuy.

Introduction: Many physicians believe age is a contraindication to lumbar fusion in elderly patients. The purpose of this study is to determine if elderly patients undergoing instrumented lumbar fusion have increased morbidity rates compared to younger patients.

Methods: The American College of Surgeons National Surgical Quality Improvement Program (NSQIP) was used to identify all patients undergoing instrumented posterolateral fusion from 2005 to 2011. Patients were stratified by decade cohorts as follows: $<65$, $65-75,75-85$, and $\geq 85$ years old. All 30 -day complications (including wound infections, cardiac events, respiratory events, returns to the operating room and others) were grouped as overall composite morbidity and were compared using multivariate analysis.

Results: A total of 1,395 patients were identified and the overall 30 -day complication rate was $11.47 \%$. The complication rates were $9.04 \%$ and $14.05 \%$ for patients less than 65 and greater than 65 , respectively. When stratified by decade cohorts, the complication rates were $9.04 \%$ for the $<65$ cohort, $13.46 \%$ for the $65-75$ cohort, $16.17 \%$ for the $75-85$ cohort, and $4.00 \%$ for the $\geq 85$ cohort. Multivariable regression analysis revealed no statistically significant difference between the $<65$ and $\geq 65$ age cohorts $(\mathrm{OR}=1.26 ; 95 \%$ CI:0.87-2.19). After stratifying into age cohorts, multivariable analyses revealed no difference in odds of postoperative morbidity for any age cohort when compared with the referent group ( $<65$ years of age). The age cohorts' odds ratios of postoperative morbidity were as follows: $65-75$ years old $(\mathrm{OR}=1.23$; $95 \% \mathrm{CI}: 0.81-1.86), 75$ - 85 years old $(\mathrm{OR}=1.58 ; 95 \%$ CI:0.98-2.55), $\geq 85$ years old (OR=0.26; 95\% CI:0.03-2.10).

Discussion: After extensive adjustment for demographic and preoperative comorbidity confounding, complication rates between older patients are shown to not be significantly different from the younger population. Therefore, age was not found to be an independent predictor of morbidity after lumbar fusion. The present data suggests that preoperative comorbidities may predict complications.

Conclusion: Patients over 65 years of age undergoing lumbar instrumented fusion do not have an increased risk of 30-day overall postoperative complications compared to patients less than 65 years of age when associated preoperative comorbidities are taken into account. 


\section{Abstracts of the 2014 Meeting of the Lumbar Spine Research Society}

Poster 34. Accuracy of Percutaneous and Open Iliac Screw Placement Using Anteroposterior Fluoroscopy

Stephen George, MD; Nathan Lebwohl, MD; Seth K. Williams, MD

University of Wisconsin School of Medicine, Department of Orthopedics and Rehabilitation

Disclosures: S. George: 2; I; Stryker. N. Lebwohl: 2; I; DePuy Synthes Spine. 4; I; DePuy Synthes Spine. S.K. Williams: 4; I; DePuy Synthes Spine.

Introduction: Iliac screw fixation has been shown to improve fusion rates, especially in deformity correction with long fusions into the sacrum. Several iliac screw fixation techniques are currently used. However, there is a paucity of literature that validates the use of a particular surgical technique. We evaluated a surgical technique that uses anterior-posterior (AP) fluoroscopy and tactile feedback with a blunt curved gearshift pedicle finder. This technique was utilized with percutaneous and open approaches. We retrospectively evaluated the accuracy of this iliac screw placement technique with post-operative CT scans.

Methods: This study is a retrospective, radiographic chart review. Charts from the two senior authors (SW and NL) were reviewed, from 2008-2013. All patients who underwent iliac screw fixation using our described technique and also had post-operative CT scans were evaluated. 57 patients, with a total of 107 iliac screws (93 open, 14 percutaneous) met the inclusion criteria. AP pelvis radiographs and CT scans were evaluated for cortical breaching of the inner and outer tables, and the sciatic notch (SN). In addition, the closest distance from the screw to the $\mathrm{SN}$ was measured.

Results: Of the 107 iliac screws, we identified three cortical breaches, all by less than $2 \mathrm{~mm}$ (1 SN breach, and 2 inner-pelvic table breaches). None of the patients with cortical breaches had resultant post-operative problems as a result. The $\mathrm{SN}$ breach was directly detected on AP radiograph. The inner table breaches were suspected on AP radiograph, by a distance greater than 2 standard deviations away from the SN. The calculated distances between the screw and the SN were statistically equal on $\mathrm{AP}$ radiographs and $\mathrm{CT}$ scans $(\mathrm{p}<0.05)$.

Discussion/Conclusion: Our described surgical technique for placement of iliac screws represents a safe and reliable option for iliac fixation in lumbosacral fusion. This technique applies to both percutaneous and open procedures. Our study validates that intraoperative AP fluoroscopy correlates closely with post-operative AP radiographs and CT scans, accurately reflecting the distance of the iliac screw to the $\mathrm{SN}$, and therefore can be used to assist with proper screw placement. Other fluoroscopic images, such as the "tear-drop" view, are not necessary.

\section{Poster 35. Neurodifferentiation of Nucleus Pulposus Stem Cells}

William M. Erwin, DC, PhD; Muhammed Z. Karim, DVM, MSc (Hons), MPhil; Milica Tanic, BSc (c); Sarah JS Kim, BSc

\section{Toronto Western Hospital/University of Toronto, Surgery}

Disclosures: W.M. Erwin: None. M.Z. Karim: None. M. Tanic: None. S.J. Kim: None.

Introduction: Injuries to the thoracolumbar spine and spinal cord, conus medullaris, and/or cauda equina can lead to bladder and bowel dysfunction and sensory/motor deficits or paralysis of the lower limbs. Treatment of such injuries requires cell replacement approaches. With a view towards possible neural repair strategies we have previously demonstrated that canine nucleus pulposus progenitor cells (NPPCs) after transplant into the brain of the shiverer mouse, can differentiate into many neural cell types including neurons, astroglial cells and oli-

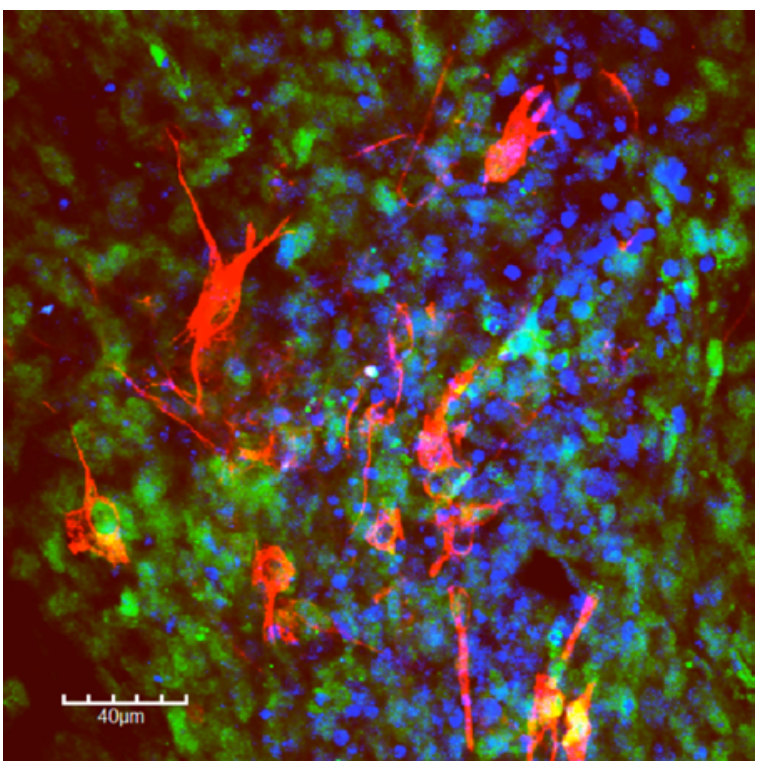

godendrocytes that form myelin within this compact myelin-deficient phenotype. Here we tested the hypothesis that soluble factors present within the brain are capable of inducing neurodifferentiation of NPPCs without the need for cell contact.

Methods: We plan to transplant neurodifferentiated NPPCs into the spinal cord, therefore we obtained NPPCs from the intervertebral discs of 12-14 week-old wild type and GFP-expressing female wistar rats (GFP cells can be tracked post tranpslant). After two passages including only colony-forming cells, we cultured the NPPCs using our novel CNS-derived neuro-differentiation medium for variable periods up to 4-weeks. At each time point we performed immunohistochemical characterization of the treated cells at 1,2,3 and 4-week intervals and examined for neuronal differentiation ( $\beta$-III tubulin and NF200), oligodendrocytes (Olig2), and astroglial cells (GFAP).

Results: NPPCS treated with CNS-conditioned media demonstrated positive staining for all markers studied. Figure 1 depicts GFP-expressing NPPCS that stain positive for the neuronal marker NF200 after two weeks of culture (red stained NF 200 positive cells). These data demonstrate that NPPCS have a robust neurodifferentiation potential and that soluble factors contained within an extract from the CNS are capable of differentiating NPPCs into multiple neurolineage cells.

Conclusions: Discectomy and spinal fusion are procedures commonly performed for the treatment of radiculopathy and spinal pain and these procedures produce significant amounts of disc material that serves no other useful purpose. Here we demonstrate that by harnessing the neurodifferentiation capacity of the ex vivo neural niche, NPPCs can be differentiated into all major classes of neural cells. This work provides the essential first steps towards the development of a novel neural repair strategy using autologous, neuro-differentiated NPPCs.

Poster 36. An Experimental Validation of a Non-Linear Finite Element Model of the Human Spine

Arin Ellingson; Hugo Giambini; Chunfeng Zhao; Ahmad Nassr; Kai-Nan An

Biomechanics Laboratory, Division of Orthopedic Research, Mayo Clinic. Rochester, $M N$

Disclosures: A. Ellingson: None. H. Giambini: None. C. Zhao: None. A. Nassr: None. K. An: None. 


\section{Abstracts of the 2014 Meeting of the Lumbar Spine Research Society}

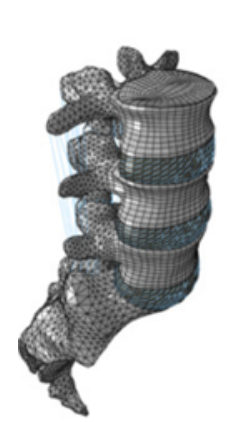

Experimental
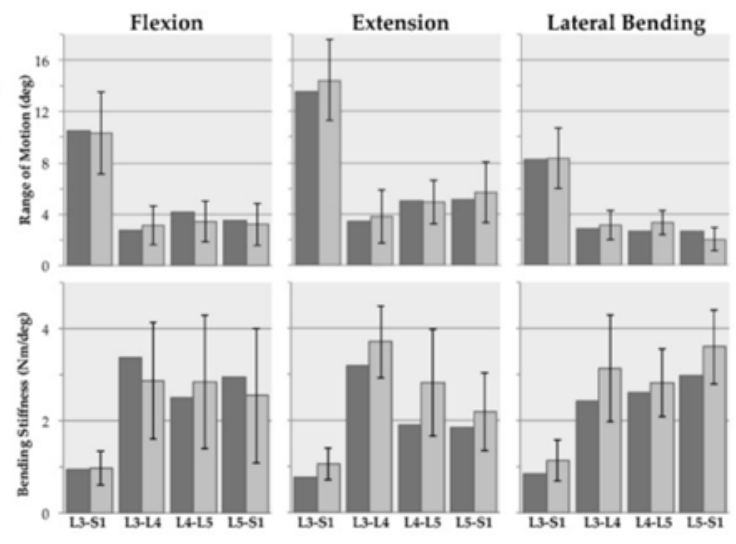

Poster 37. Ten-Year Outcomes of Lumbar Spine Fusion Surgery at an Academic, Tertiary Hospital

AbdulRahman A. Abutaleb, BS; Jason W. Savage, MD; Wellington K. Hsu, MD; Alpesh A. Patel, MD FACS

Northwestern University Feinberg School of Medicine, Department of Orthopaedic Surgery

Disclosures: A.A. Abutaleb: None. J.W. Savage: None. W.K. Hsu: None. A.A. Patel: None.

Introduction: Lumbar fusion is a common procedure performed in the United States, which is complicated by an aging patient population. This study aims to determine the demographics and comorbidities that increase the risk of post-operative complications, length of stay (LOS), and readmissions by analyzing patients that underwent lumbar fusion surgery at a tertiary

Introduction: Finite element (FE) models of the lumbar spine are a powerful tool to non-invasively investigate the biomechanical responses of clinical conditions. These range from alterations from pathology, including degeneration, to implants and fixation device design. The purpose herein was to develop, optimize, and validate a lumbar spine FE model.

Methods: A nonlinear, three-dimensional lumbar spine (L3-S1) FE model was developed based on the CT scan of a 52-year-old male using Mimics (v14.12-Materialise) and HyperMesh (v10.0-Altair Engineering). The model was evaluated with Abaqus (v6.12-Dassault Systèmes). The following ligaments were implemented and modeled hypoelastically: anterior longitudinal ligament, posterior longitudinal ligament, facet capsular ligament, ligamentum flavum, intertransverse ligament, interspinous ligament, and supraspinous ligament. The disc was compartmentalized into the nucleus pulposus (NP) and annulus fibrosus (AF). The NP was modeled as linearly elastic with modulus $1 \mathrm{MPa}$ and Poison's ratio of 0.4999. The AF ground substance was modeled as a neo-Hookean solid $(\mathrm{C} 10=0.348, \mathrm{D} 1=0.3)$ with seven reinforced tensile fiber layers oriented at $\pm 30^{\circ}$ and whose linear elastic properties varied by radial position.

Experimental validation utilized 18 cadaveric osteoligamentous lumbar spines from L1 to Sacrum (53.2 \pm 15.5 yrs.). In a six-axis spine simulator (8821 Biopuls-Instron), specimens underwent flexionextension and lateral bending. Pure moments of up to $7 \mathrm{Nm}$ were applied superiorly, while allowing inferior translation minimizing shear forces. Intersegmental displacements were recorded using a 5-camera motion analysis system (Vicon MX-F40NIR-Vicon Motion Systems) capturing an infrared reflecting marker-set attached to each vertebrae. Loading and boundary conditions of the FE analysis mimicked the experimental protocol. Range of motion (ROM) and stiffness were measured and used for validation.

Results: Figure 1 depicts comparative output data for the FE model and summary statistics from the experimental measurements. Intersegmental ROM and stiffness were analogous - within one standard deviation of the mean - for all levels during all directions of bending.

Conclusion: We have developed and optimized a robust FE model of the lumbar spine. The model exhibited similar overall motion patterns, including comparable ROM and stiffness. This model can be used in subsequent studies to understand how spinal pathology and the incorporation of various fixation devices affect the biomechanical response. academic medical center.

Methods: An institutional patient database (Electronic Data Warehouse) was retrospectively reviewed over the years 2003 to 2013 for patients that underwent lumbar fusion surgery using ICD-9 and CPT procedural codes. Results were reported as differences in operating room (OR) time and LOS, while adverse outcomes were measured by change in the odds ratio (COR) compared to a "control" group (white males, 30-40 years old, BMI between 18.5-25, and no documented comorbidities). $\mathrm{P}<0.001$ was considered significant.

Results: In 2902 patients who underwent lumbar fusion surgery urinary complications $(\mathrm{n}=113,3.89 \%)$, urinary tract infections $(n=108,3.72 \%)$, and deep vein thrombosis $(n=105,3.62 \%)$ were the most common complication. Using the Charlson Comorbidity Index as a predictor of complications, for every point increase in the comorbidity index there was a significant increase in thirty-day (0-30 day) and sixty-day (31-60 day) readmission, pneumonia, urinary tract infections, urinary complications and gastrointestinal bleed (Table1). For every point increase in Charlson Index the OR time decreased by 10.023 minutes $(\mathrm{p}=0.001)$ compared to our control population (average 338 minutes). The predictors for increased LOS included age between 50 and 69 years ( 35.843 hours; $\mathrm{p}<0.001)$, age over 70 years (43.357 hours; $\mathrm{p}<0.001)$ and metastatic solid tumor (111.848 hours; $\mathrm{p}<0.001)$ compared to the control population with a LOS of 139.43 hours (5.81 days). Conversely, smokers had a significant decrease in LOS by 18 hours $(\mathrm{p}=0.001)$.

Discussion/Conclusion: An analysis of LOS for lumbar fusion surgery showed an estimated stay of 5.8 days for the control population,

\begin{tabular}{|l|r|r|}
\hline \multicolumn{3}{|c|}{ Charlson Comorbidity Index as a Predictor } \\
\hline Peri/Postoperative Outcomes & \multicolumn{1}{|c|}{ Coefficient } & \multicolumn{1}{l|}{ P value } \\
\hline OR time (mins) average $=338.11$ & -10.023 & 0.001 \\
\hline length of stay (hours) average $=139.43$ & 2.674 & 0.355 \\
\hline readmissions 0-30 days & 0.268 & $<0.001$ \\
\hline readmissions 31-60 days & 0.374 & $<0.001$ \\
\hline Pneumonia & 0.266 & $<0.001$ \\
\hline UTI & 0.239 & $<0.001$ \\
\hline Wound Infection & 0.067 & 0.563 \\
\hline Spine Complications & -0.961 & 0.248 \\
\hline Dysphagia & 0.111 & 0.225 \\
\hline Urinary Complications & 0.260 & $<0.001$ \\
\hline GI Bleed & 0.278 & 0.001 \\
\hline Acute Renal Failure & 0.225 & 0.022 \\
\hline Mechanical Complications of Implant & 0.121 & 0.366 \\
\hline
\end{tabular}

Table 1: Charlson Comorbidity Index as a predictor of peri/postoperative complications of lumbar fusion surgery. Only factors that showed statistical significance are highlighted above. The Operating Room Time and Length of stay show the incremental increase in time for every 1 point increase in the patient's Charlson Index. The other complications are reported as an increase or decrease in the odds ratio (OR) for developing the complication. 


\section{Abstracts of the 2014 Meeting of the Lumbar Spine Research Society}

with LOS increased for patients with age $>50$ years and metastatic solid tumor, while one's smoking status decreased LOS. Recognizing patients with comorbidities and demographics addressed in this study that increase OR time, LOS, complications and readmissions for lumbar fusion surgery can better help direct physician pre- and perioperative decisions.

\section{Poster 38. Ten-Year Outcomes of Thoracolumbar Spine Fusion} Surgery at an Academic, Tertiary Hospital

AbdulRahman A. Abutaleb, BS; Jason W. Savage, MD; Wellington K. Hsu, MD; Alpesh A. Patel, MD FACS

Northwestern University Feinberg School of Medicine, Department of Orthopaedic Surgery

Disclosures: A.A. Abutaleb: None. J.W. Savage: None. W.K. Hsu: None. A.A. Patel: None.

Introduction: While the rate of spinal surgery in the US is $40 \%$ more than any other country, the outcomes of surgery remain difficult to predict. The purpose of this study is to determine clinical factors that correlate with complications, length of stay (LOS), and hospital readmissions among patients undergoing thoracolumbar fusion spine surgery at a single, tertiary, academic medical center.

Methods: A patient database at an academic institution (Electronic Data Warehouse) was retrospectively reviewed over the years 2003 to 2013 for patients that underwent thoracic and/or lumbar fusion surgery using ICD 9 and CPT procedural codes. Results were reported as changes in time for operating room (OR) time and LOS, while adverse outcomes were measured by change in the odds ratio (COR) compared to a "control" group (white males, 30-40 years old, BMI between 18.5-25, and no documented comorbidities). $\mathrm{P}<0.001$ was considered significant.

Results: In 3605 patients, deep vein thrombosis (DVT) ( $\mathrm{n}=160$, $4.43 \%)$, urinary complications $(\mathrm{n}=158,4.38 \%)$, and urinary tract infections $(n=149,4.13 \%)$ were the most common complications. A higher Charlson Comorbidity Index correlated with a significant

\begin{tabular}{|l|r|r|r|r|}
\hline Charlson Comorbidity Index Predictor \\
\hline Peri/Postoperative Outcomes & Coefficient & P value & 95\% C.I. \\
\hline OR time (mins) average $=349.8186$ & -3.787 & 0.068 & -7.853 & 0.279 \\
\hline LOS (hours) average $=173.4977$ & 5.884 & 0.015 & 1.165 & 10.602 \\
\hline readmissions 0-30 days ( $=300,8.32 \%)$ & 0.181 & $<0.001$ & 0.116 & 0.247 \\
\hline readmissions 31-60 days ( $\mathrm{n}=101,2.77 \%)$ & 0.298 & $<0.001$ & 0.219 & 0.377 \\
\hline Deep Vein Thrombosis & 0.157 & $<0.001$ & 0.069 & 0.244 \\
\hline Urinary Complications & 0.166 & $<0.001$ & 0.080 & 0.253 \\
\hline Urinary Tract Infection & 0.164 & $<0.001$ & 0.076 & 0.253 \\
\hline Wound Infection & 0.113 & 0.048 & 0.001 & 0.225 \\
\hline Dysphagia & 0.102 & 0.083 & -0.013 & 0.216 \\
\hline Pneumonia & 0.207 & $<0.001$ & 0.109 & 0.305 \\
\hline Malunion & -0.323 & 0.131 & -0.741 & 0.096 \\
\hline Acute Renal Failure & 0.231 & $<0.001$ & 0.127 & 0.335 \\
\hline Pulmonary Complications & 0.217 & $<0.001$ & 0.102 & 0.332 \\
\hline Gastrointestinal bleed & 0.225 & $<0.001$ & 0.109 & 0.340 \\
\hline Delirium & 0.312 & $<0.001$ & 0.206 & 0.418 \\
\hline Spinal Cord Injury & -0.487 & 0.244 & -1.306 & 0.333 \\
\hline Pulmonary Embolism & 0.363 & $<0.001$ & 0.261 & 0.465 \\
\hline Spine Complications & 0.172 & 0.019 & 0.028 & 0.316 \\
\hline Mechanical Complications of Implant & 0.070 & 0.454 & -0.113 & 0.254 \\
\hline Hemorrhage/Hematoma & 0.283 & $<0.001$ & 0.148 & 0.418 \\
\hline
\end{tabular}

Table 1: Charlson Comorbidity Index as a predictor of peri/postoperative complications of thoracolumbar fusion surgery. Only factors that showed statistical significance are highlighted above. The Operating Room Time and Length of stay show the incremental increase in time for every 1 point increase in the patient's Charlson Index. The other complications are reported as an increase or decrease in the odds ratio (OR) for developing the complication compared to the control population. C.I. indicates Confidence Interval. increase in thirty-day (0-30) and sixty-day (31-60) readmission, DVT, urinary complications, urinary tract infection, pneumonia, acute renal failure, pulmonary complication, gastrointestinal bleed, delirium, pulmonary embolisms, hemorrhage/hematoma (Table 1). LOS significantly increased for patients between 50 and 69 years old (39.676 hours; $\mathrm{p}<0.001)$, over 70 years old $(50.263$ hours; $\mathrm{p}<0.001)$, and underweight patients with a BMI between 10 and 18.5 (109.4 hours; $\mathrm{p}<0.001$ ), while smokers spent 22 hours less in the hospital. OR time decreased significantly for patients without a known or documented race, by 37.84 minutes. Patients over 70 years old had a significant increase of $.787 \mathrm{COR}$ for thirty-day readmission, while metastatic solid tumor had an increased 2.22 COR for sixty-day readmission.

Discussion/Conclusion: In this study we identified the risk factors for increased complications, LOS, OR time and readmissions after thoracolumbar fusion surgery such as increasing age and Charlson Comorbidity Index. We hope that this information can help physicians form a more informed and thorough pre-operative assessment of patient comorbidities and demographics in order to reduce patient morbidity and hospital costs.

Poster 39. Which Patients Get Admitted And Readmitted After Elective Lumbar Discectomy? An Analysis Of 3,394 ACS-NSQIP Patients

Bryce A. Basques, BS; Nicholas S. Golinvaux, BA; Daniel D. Bohl, $\mathrm{MPH}$; Jonathan N. Grauer, MD

\section{Yale School of Medicine, Orthopaedics and Rehabilitation}

Disclosures: B.A. Basques: None. N.S. Golinvaux: None.D.D. Bohl: None. J.N. Grauer: 2; I; Stryker. 4; I; Affinergy, Alphatec, Bioventus, Depuy, Harvard Clinical Research Institute, Powered Research, Stryker, Transgenomic.

Introduction: Lumbar discectomy is a common surgical procedure that can be performed as an outpatient procedure or be associated with a short admission. Information regarding patient characteristics associated with postoperative admission and post-discharge readmission is

limited. This information would be useful for patient counseling and postoperative planning.

Methods: Using the American College of Surgeons National Surgical Quality Improvement Program (ACS-NSQIP) database for the year 2012, a retrospective cohort study was performed. Patients undergoing elective lumbar discectomy were identified using International Classification of Diseases, 9th Revision (ICD-9) and Current Procedural Terminology (CPT) codes.

Admission to the hospital was defined as a postoperative length of stay (LOS) lasting one day or more. Readmission was defined as when a patient had an unplanned readmission one or more times within 30 days of surgery. Patient characteristics were tested for association with admission and readmission using bivariate and multivariate logistic regressions.

Results: A total of 3,394 elective lumbar discectomy cases were identified. The average age was $48.9 \pm 14.7$ years (mean \pm standard deviation). A total of 1,940 patients (57.2\%) were admitted to the hospital following surgery. A total of 86 patients $(2.5 \%)$ were readmitted to the hospital following discharge.

Patient characteristics independently associated with admission after discectomy were age (40-49 years Odds Ratio $[\mathrm{OR}]=0.9,50-59$ years $\mathrm{OR}=1.1, \geq 60$ years $\mathrm{OR}=1.3 ; \mathrm{p}=$ $0.001)$, female sex $(\mathrm{OR}=1.3, \mathrm{p}=0.001)$, American Society of Anesthesiologists class (ASA) class 3-4 $(\mathrm{OR}=1.3, \mathrm{p}=0.002)$, preoperative hematocrit $<36.0(\mathrm{OR}=1.5, \mathrm{p}=0.015)$, and dependent functional status $(\mathrm{OR}=4.3, \mathrm{p}=0.003)$.

ASA class 3-4 $(\mathrm{OR}=1.8, \mathrm{p}=0.014)$, history of pulmonary disease $(\mathrm{OR}=2.4, \mathrm{p}=0.008)$, and occurrence of any inpatient complication $(\mathrm{OR}=4.7, \mathrm{p}=0.015)$ were independently associ- 


\section{Abstracts of the 2014 Meeting of the Lumbar Spine Research Society}

ated with readmission after lumbar discectomy. The most common reason for readmission was infection (15 patients were readmitted for infection: $17.4 \%$ of the total readmitted [86 patients] or $0.4 \%$ of the total discectomy cohort).

Conclusions: Older, female patients with increased ASA class, preoperative anemia, or dependent functional status were more likely to be admitted following lumbar discectomy. Readmission was more likely for patients with increased ASA class, a history of pulmonary disease, or those who had any inpatient complication. Patients and providers should be aware of these factors in order to have accurate expectations after lumbar discectomy.

Poster 40. Application Of Prophylactic Vancomycin Powder In Spine Fusion Operations And Reltionship To The Likelihood Of Postoperative Wound Infection

Jim A. Youssef; Douglas Orndorff, MD; Morgan Scott; Rachel Ebner; Allison Knewitz

\section{Durango Orthopedic Assoc/Spine Colorado}

Disclosures: J.A. Youssef: 1; I; Nuvasive, Integra, Amedica, Osprey Biomedical. 4; I; Nuvasive, Amedica, Integra. 6; I; Amedica, Vertiflex, Benvenue, Paradigm Spine, Promethean Surgical, ISD, Spinicity, Spinal Ventures. 7; I; Nuvasive, Vertiflex, Globus Medical, Integra. D. Orndorff: 1; I; Integra. 4; I; Nuvasive, Amedica, Stryker, Integra. 7; I; Nuvasive, Integra, Globus, Vertiflex. M. Scott: None. R. Ebner: None. A. Knewitz: None.

Introduction: Even with low overall rates of surgical site infections (SSI) following open lumbar fusion procedures (0\%-12.7\%), use of the powder form of the antibiotic vancomycin has become more routine due to certain types of IV prophylaxis being considered insufficient in hospitals. With increasing use, there are concerns about the development of strains of methicillin-resistant staphylococcus aureus (MRSA) that are resistant to vancomycin. This study is aimed to evaluate whether using one gram of vancomycin powder placed in the deep space of soft tissues at the completion of open lumbar procedures prior to closure is effective in reducing post-operative wound infections as compared to the standard of care at $\leq 6$ weeks and $\leq 3$ months post-operatively.

Methods: In this IRB approved, retrospective, single center analysis, 220 patients were included who received open lumbar spine surgery (TLIF, PSF, XLIF/PSF) between January of 2012 and June of 2013. 88 patients $(40 \%)$ received vancomycin powder intraoperatively placed into the deep space of the soft tissue prior to closure and/or mixed with the bone graft and placed in the deep space, and 132 patients did not. All patients received pre-operative intravenous antibiotics per protocol as well as standard antibiotic IV administration peri-operatively.

Results: Among both the patients who received vancomycin powder and those that did not, 0 patients experienced wound infection at $\leq 6$ weeks or $\leq 3$ months post-operatively. No important differences were indicated among the covariates for each group.

Discussion: Since 2002, cases have been reported of vancomycinresistant S. aureus (VRSA). Local application of the vancomycin may be less likely to promote antibiotic resistance relative to intravenous

Tabled.

\begin{tabular}{l|cc|}
\multicolumn{1}{c}{+ +Vancomycin } & -Vancomycin \\
\hline Number of patients & 88 & 132 \\
Average BMI & 28.4 & 27.8 \\
Number of Tobacco users & 15 & 20 \\
\cline { 2 - 3 } Number MaleFemale & $41 / 47$ & $63 / 69$ \\
Average \# Levels treated & 2.0 & 2.1 \\
EBL & 287.7 & 291.6 \\
OR Time (min) & 286 & 299 \\
Length of stay & 2.3 & 2.8 \\
\cline { 2 - 3 }
\end{tabular}

administration due to poor systemic absorption of vancomycin. Additional local antibiotic treatment may be unnecessary.

Conclusions: Infection rates within 3 months for both +/Vancomycin did not occur in this setting, carrying a type 2 statistical error. This extremely low infection rate for both groups may indicate that the effort to reduce SSI occurrence with routine vancomycin powder may not be indicated for in straightforward lumbar spine procedures. A larger cohort may be necessary to further elucidate whether vancomycin should be indicated.

Poster 41. Retraction Time Per Level And Postoperative Leg Pain Following Extreme Lateral Interbody Fusion

Jim A. Youssef; Douglas Orndorff, MD; Morgan Scott, MS; Rachel Ebner, MS

\section{Durango Orthopedic Assoc/Spine Colorado}

Disclosures: J.A. Youssef: 1; I; NuVasive, Integra, Amedica, Osprey Biomedical. 4; I; Nuvasive, Amedica, Integra. 6; I; Amedica, Vertiflex, Benvenue, Paradigm spine, Promethean Surgical, ISD, Spinicity, Spinal Ventures. 7; I; Nuvasive, Integra, Vertiflex, Globus Medical. D. Orndorff: 1; I; Integra. 4; I; Nuvasive, Integra, Stryker, Amedica. 7; I; Nuvasive, Integra, Vertiflex, Globus Medical. M. Scott: None. R. Ebner: None.

Introduction: Although XLIF as an effective treatment for spinal disorders is increasing in popularity, postoperative leg pain and/or dysesthesia (PLPD) are potential complications of this procedure. We report on the incidence of PLPD as related to intra-operative psoas retraction times for an XLIF procedure.

Methods: Local IRB approved, prospective, non-randomized single center study of patients who underwent XLIF procedures from February 2011 to July 2013. Retraction times were collected intraoperatively for 49 patients ( 84 operative levels). Patient reported PLPD, collected at 2-week, 6-week and 1-year follow up visits were categorized; category one is isolated numbness/dysesthesia without pain or weakness; category 2 is numbness plus pain on contraction resulting in poor effort, but not weakness; category 3 is numbness and/or weakness including psoas weakness with motor symptoms (grade (0-5)). Differences in retraction time were analyzed between those that experienced PLPD and those that did not using two-sample t-tests.

Results: 49 patients (84 levels) were analyzed at the 2 and 6 week time, with 21 patients (39 levels) analyzed at 1 year. Average retraction time in minutes per level was recorded as $22 \mathrm{~min}$ for T12-L1, 18.5 min at L1-2, $23.2 \mathrm{~min}$ at L2-3, 23.3min at L3-4 and $24.2 \mathrm{~min}$ at L4-5. Retraction times for those that did not have post operative symptoms in any of the three categories were significantly different than those that did ( $p$-value $=0.0198$ ); with a mean of 21.7 min compared to $25.1 \mathrm{~min}$. At the 2-week follow-up, 5/49 patients (10.2\%)

\section{Average Retraction Times per Level}

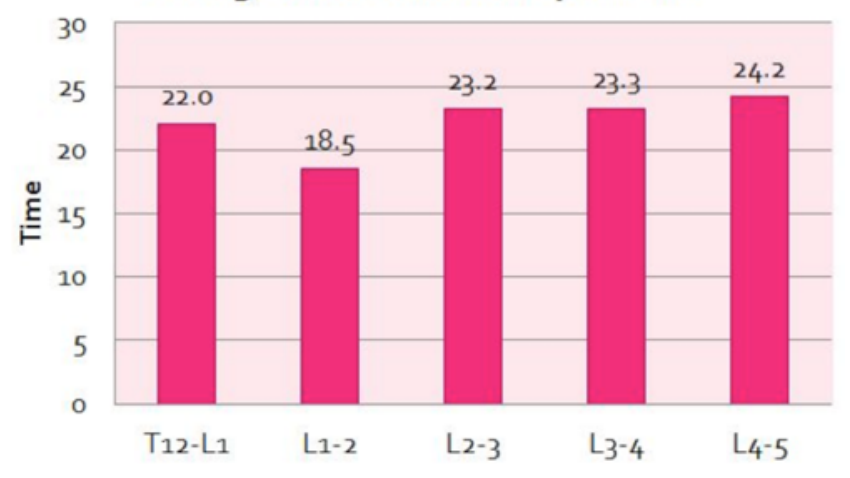




\section{Abstracts of the 2014 Meeting of the Lumbar Spine Research Society}

reported category one symptoms, $8 / 49$ patients $(16.3 \%)$ reported category two symptoms and $6 / 49$ patients $(12.2 \%)$ reported category three symptoms. At 6-weeks, $2 / 49$ patients $(4.1 \%)$ reported category one symptoms, $1 / 49$ patients $(2.0 \%)$ reported category two symptoms, and $4 / 49$ patients $(8.2 \%)$ reported category three. At 1-year, no patients continued to report symptoms within the three previously mentioned categories.

Conclusion: $54.5 \%$ of PLPD due to the XLIF procedure resolved by 6 -weeks, with $100 \%$ resolution by one year for the 21 patients who achieved 1-year follow-up. Our data suggests that retraction times less than 22 minutes may decrease rates of PLPD.

Poster 42. CT Attenuation Coefficient (Hounsfield Unit) To Estimate Bone Mineral Density T-score

Nathan R. Hendrickson, MS; Paul A. Anderson, MD, MS

University of Wisconsin School of Medicine and Public Health, Orthopedics and Rehabilitation

Disclosures: N.R. Hendrickson: None. P.A. Anderson: 1; I; Stryker, Pioneer Surgical. 4; I; Stryker. 6; I; Pioneer Surgical, Titan Surgical, SPARTEC, Expanding Orthopedics, SI Bone. 9; I; Journal of Bone and Joint Surgery.

Introduction: Clinical computed tomography (CT) studies performed for other indications can be used to opportunistically assess vertebral bone with no additional radiation or cost to the patient. Reference values for young women are needed to evaluate changes in these values across the lifespan and to calculate T-scores, which is a metric used to make clinical decisions. Our purpose was to determine reference values for lumbar CT attenuation (in Hounsfield units [HU]) and determine the validity of $\mathrm{HU} \mathrm{T}$-scores for identifying patients with osteoporosis.

Methods: This retrospective cohort study determined reference values for lumbar trabecular CT attenuation from L1 to L4 (in Hounsfield units [HU]) from 190 young women $(24.0 \pm 2.9$ yrs $)$. Reference values were used to calculate T-scoreHU in a validation cohort of 252 older patients ( $58.9 \pm 7.5$ yrs) undergoing CT and DXA within a 6-month period. T-scoreHU was compared to T-scoreDXA to determine validity of $\mathrm{HU}$ to identify patients with osteoporosis. Receiver operating characteristic curves were used to assess sensitivity and specificity.

Results: Reference group HU ranged from $227 \pm 42$ at L3 to 236 \pm 42 at $\mathrm{L} 1(\mathrm{P}<0.001)$. Validation group $\mathrm{T}$-scoreDXA was $-0.7 \pm 1.5$ and $-0.9 \pm 1.2$ at lumbar and femoral sites respectively. Mean T-scoreHU was -2.3. A T-scoreHU of -3.0, corresponding to $110 \mathrm{HU}$, was $48 \%$ sensitive and $91 \%$ specific for osteoporosis in the validation group with ROC area under the curve ranging from 0.825 to 0.853 depending on lumbar level assessed.

Discussion: Although T-scores calculated from lumbar trabecular HU are lower than T-scores from DXA, T-score thresholds can be selected to achieve either high sensitivity or high specificity when screening for osteoporosis.

Conclusions: T-scores obtained from CT studies performed for other indications can be used to identify patients that may benefit from additional studies or treatment. HU T-scores may also provide a tool to assess changes in vertebral bone and the relationship to fracture risk across the lifespan.

Poster 43. Mechanical Allodynia following Experimental Disc Herniation involves Intraneural Macrophage Migration and is Blocked by Supraphysiologic Systemic Selenium Delivery

Mohammed F. Shamji, MD, PhD; Yu-Shan Tu, MSc; Michael W. Salter, MD, PhD

\section{Toronto Western Hospital, Neurosurgery}

Disclosures: M.F. Shamji: None. Y. Tu: None. M.W. Salter: None.

Introduction: Disc-herniation induced radiculopathy arises from both mechanical compression and biochemical inflammation of apposed neural elements. This study demonstrated the need for intraneural macrophage migration after placement heterotopic disc tissue to generate the painful neuropathy phenotype.

Methods: C57BL/6 mice underwent a surgical procedure with mid-thigh exposure of the sciatic nerve. Control animals underwent exposure only $(\mathrm{n}=12)$ and experimental animals underwent placement of littermate tail nucleus pulposus $(n=12)$. Animals were evaluated throughout one week for mechanical allodynia by Von Frey testing, thermal hyperalgesia by heat withdrawal latency, cold allodynia by acetone testing, and gait stability by RotaRod testing. At sacrifice, immunohistochemistry was performed to identify perineural and intraneural macrophage and lymphocyte presence. Necessity of an inflammatory response was tested by attenuating systemic inflammation with intraperitoneal selenium delivery.

Results: Mice exposed to heterotopic NP stimulation demonstrated substantial mechanical allodynia maximal through the first five days and resolving by three weeks after surgery $(0.014 \pm 0.007 \mathrm{~g}(\mathrm{NP})$ vs. $1.0 \pm 0.3 \mathrm{~g}$ (sham), $\mathrm{p}<0.05$ ). Associated thermal hyperalgesia and cold allodynia also occurred. Substantial intraneural macrophage infiltration was observed in this group, alongside associated CD3+ IL17+ autoreactive lymphocytes at the disc-nerve interface (Figure 1, p < $0.01)$ Systemic selenium administration blocked this behavioral phenotype in the acute inflammatory phase, also affecting the extent of macrophage extravasation.

Conclusions: Non-compressive disc herniation leads to altered behavior in this animal disease model, with demonstrated need for intraneural macrophage migration. Strategies to decrease perineural inflammation or maintain integrity of the blood nerve barrier may be effective in treating painful disc-herniation radiculopathy.

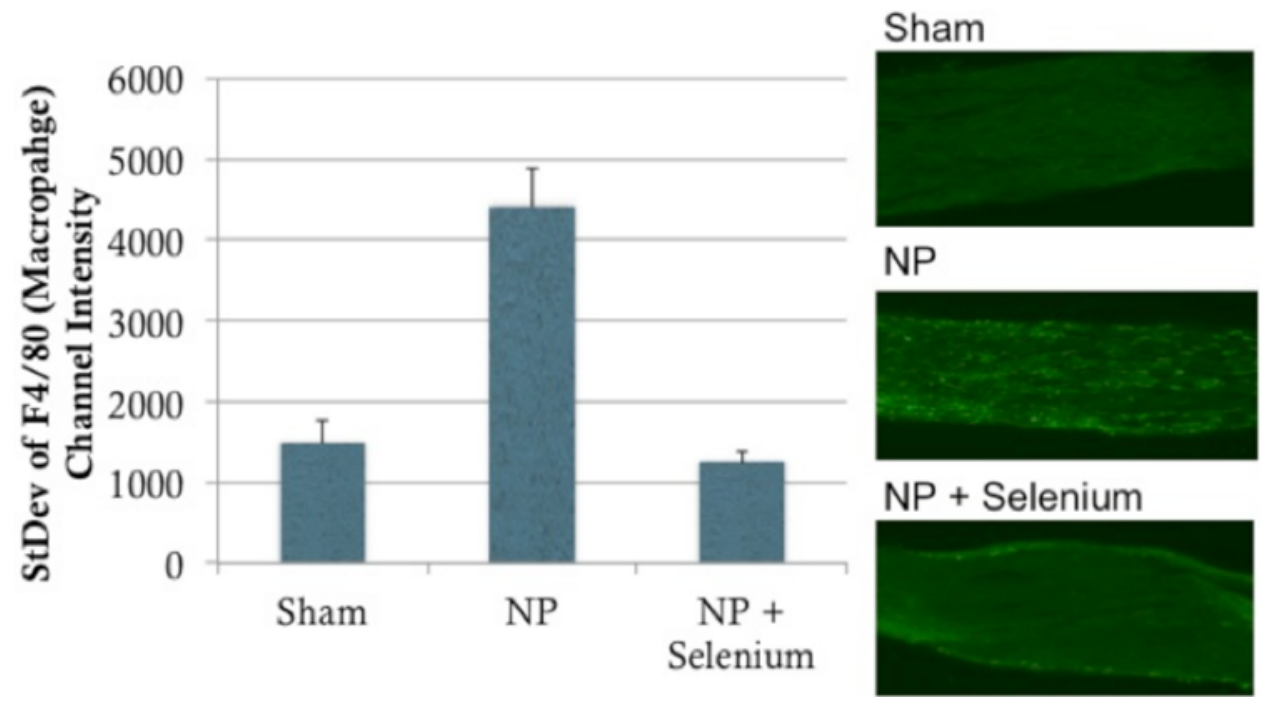




\section{Abstracts of the 2014 Meeting of the Lumbar Spine Research Society}

\section{Poster 44. Midterm self-reported Quality Of Life Outcomes After Spine Surgery For Lumbar Radiculopathy}

Stelios Koutsoumbelis; Alex Richter; David essig; Sara Merwin; Jeff Silber

NSLIJ, Orthopaedic Surgery

Disclosures: S. Koutsoumbelis: None. A. Richter: None. D. essig: None. S. Merwin: None. J. Silber: None.

Introduction: Lumbar radiculopathy (LR) is a source of significant morbidity and economic burden, with U.S. prevalence estimated between at between $3-5 \%$, and symptoms usually first appearing in middle age.

Methods: In a single center follow-up survey study at a tertiarycare teaching hospital. Inclusion: adults, ICD-9 codes consistent with lumbar radiculopathy. Exclusions: minors, revisions, less than two year follow up period. Oswestry Disability Index (ODI) were mailed to patients meeting inclusion criteria. Variables include: surgery type, symptoms, medications, subsequent surgeries and demographics. Outcome measures, functionality, and quality of life ratings were measured. Telephone administration of the questionnaire is the final phase to capture data from patients not returning surveys.

Results: Surveys were sent to 339 patients meeting inclusion. Two patients $(0.9 \%)$ elected to withdraw. The patient population was $57.5 \%$ male with a mean age of 40.4 years $(\mathrm{SD}=11.4)$. The mean follow up time was 6.5 years $(\mathrm{SD}=2.1)$. Laminectomy alone was performed in 16 patients, laminectomy with discectomy in 281 , and laminectomy/discectomy with fusion in 42 patients.

Pre-operatively $87.2 \%$ and $84 \%$ of patients rated back and leg pain respectively as 7 out of 10 or greater. Postoperatively this improved to $8.8 \%(\mathrm{p}<0.0001)$ for back pain and $4.0 \%(\mathrm{p}<0.0001)$ for leg pain. Eight patients (6.4\%) required additional surgery. 27 patients $(21.6 \%)$ continued to use analgesic medication with 16 $(59.2 \%)$ using NSAIDs and $8(29.6 \%)$ using narcotic medications. The ODI score averaged $13.9 \%$, corresponding to mild disability.

Conclusions: The majority of this operative cohort reported a substantial decrease in pain after surgery and stated that disability was in the mild range. Less than $5 \%$ of patients required further surgery.

Poster 45. Minimally-Invasive Transforaminal Lumbar Interbody Fusion Versus Open Lumbar Laminectomy and Fusion: Blood Loss, Surgical Complications, and Length of Hospital Stay

Matthew Zfass-Mendez, MD; Amar A. Patel, MD; Barth A. Green, MD; Nathan H. Lebwohl, MD; Allan D. Levi, MD; Steven Vanni, DO; Seth K. Williams, MD

University of Wisconsin School of Medicine, Department of Orthopedics and Rehabilitation

Disclosures: M. Zfass-Mendez: None. A.A. Patel: None. B.A. Green: None. N.H. Lebwohl: 1; I; DePuy Synthes Spine. 2; I; DePuy Synthes Spine. 4; I; DePuy Synthes Spine. A.D. Levi: 2; I; DePuy Synthes Spine, Medtronic Spine. S. Vanni: 4; I; DePuy Synthes Spine, NuVasive. S.K. Williams: 4; I; DePuy Synthes Spine.

Introduction. Posterolateral fusion (PLF) is used to provide stability across mobile spinal segments, with fusion occurring between the transverse processes. Posterior interbody fusion techniques theoretically provide additional stability by adding anterior column support and fusion across the disk space. Two of the most commonly used interbody fusion techniques are open PLIF (posterior lumbar interbody fusion) and MIS TLIF (minimally-invasive transforaminal lumbar interbody fusion). This study was designed to determine if the data support the theoretical advantages of MIS TLIF over the open

\begin{tabular}{|l|c|c|c|c|}
\hline & EBL (mL) & Pts transfused & Complications & $\begin{array}{c}\text { Hospital stay } \\
\text { (days) }\end{array}$ \\
\hline Open PLF & 313 & $29 \%$ & $14.6 \%$ & 4.3 \\
\hline Open PLIF & 514 & $43 \%$ & $9.5 \%$ & 4.8 \\
\hline MIS TLIF & 136 & $0 \%$ & $1.4 \%$ & 4.1 \\
\hline
\end{tabular}

surgical techniques with respect to blood loss, surgical complications, and length of hospital stay.

Methods. Operative reports and postoperative data of consecutive patients undergoing single-level, primary open PLF $(n=41)$, open PLIF ( $n=42)$, and MIS TLIF $(n=71)$ by 6 seven surgeons were retrospectively evaluated. The patient's body mass index (BMI), age at surgery, gender, operative blood loss, use of transfusion products, surgical complications (wound infection, screw misplacement, dural tear, neurological injury), and length of stay were tabulated. Patient data were controlled for age, BMI, and gender for statistical analysis.

Results. Patients undergoing open PLF and open PLIF respectively experienced a significantly higher blood loss $(\mathrm{OR}=2.4,95 \%$ CI 1.9 3.1; $\mathrm{OR}=4.2,95 \%$ CI 3.3-5.3), higher volume of blood transfusion $(\mathrm{OR}=67.9,95 \%$ CI $16.8-395.6 ; \mathrm{OR}=257.1,95 \%$ CI 50.6-1949.7), higher volume of cell saver transfusion $(\mathrm{OR}=16.7,95 \% \mathrm{CI} 4.8-80.8$; $\mathrm{OR}=64.7,95 \%$ CI 16.0-368.7), and more surgical complications $(\mathrm{OR}=14.4,95 \% \mathrm{CI}=2.6-174.1 ; \mathrm{OR}=5.5,95 \% \mathrm{CI}=0.8-72.2)$ than those undergoing MIS TLIF. There was no statistically significant difference in duration of hospital stay.

Conclusions. MIS TLIF was performed with less intraoperative blood loss, a lower transfusion rate, and fewer surgical complications than the open fusion techniques, without a significant effect on duration of hospital stay.

Poster 46. Comparison Of 372 Patients Undergoing Decompression For Degenerative Spondylolisthesis From The SPORT Trial With 1,035 From The ACS NSQIP Database

Nicholas S. Golinvaux, BA; Bryce A. Basques, BS; Daniel D. Bohl, MPH; Jonathan N. Grauer, MD

\section{Yale University School of Medicine, Department of Orthopaedics}

Disclosures: N.S. Golinvaux: None. B.A. Basques: None. D.D. Bohl: None. J.N. Grauer: 2; I; Stryker. 4; I; Affinergy, Alphatec, Bioventus, Depuy, Harvard Clinical Reserach Institute, Powered Research, Stryker, Transgenomic.

Introduction: The Spine Patient Outcomes Research Trial (SPORT) was a multi-armed, prospective, randomized clinical trial. The degenerative spondylolisthesis arm of this study had both observational and randomized cohorts to evaluate surgical versus non-surgical care.

Recent use of national databases for clinical spine research offers an avenue for evaluating the generalizability of SPORT data to current clinical practice and outcomes.

Methods: Using current procedural terminology (CPT) and International Classification of Diseases, 9th revision (ICD-9) coding, we identified all elective lumbar laminectomies performed for degenerative spondylolisthesis in the American College of Surgeons National Surgical Quality Improvement Program (ACS NSQIP) database from 2010-2012. This database includes hospital and 30-day follow-up data.

Where possible based on published data, demographics, perioperative factors, and complications were compared between SPORT and NSQIP. The two surgical SPORT groups (randomized and observational) were combined during analysis of perioperative factors and complication rates.

Results: A total of 1,035 decompressions for spondylolisthesis were identified in NSQIP and were compared to the 372 patients from SPORT who underwent surgery (172 randomized, 200 observational). 


\section{Abstracts of the 2014 Meeting of the Lumbar Spine Research Society}

Table 1: Demographics of the patient populations

\begin{tabular}{|c|c|c|c|c|c|}
\hline & $\begin{array}{c}\text { SPORT } \\
\text { Randomized cohort }\end{array}$ & $\begin{array}{c}\text { SPORT } \\
\text { Observational cohort }\end{array}$ & $\begin{array}{c}\text { SPORT } \\
\text { Patients treated } \\
\text { with surgery* }\end{array}$ & $\begin{array}{c}\text { SPORT } \\
\text { Paticnts not treated } \\
\text { with surgery* }\end{array}$ & NSQIP \\
\hline & $\mathrm{n}(\%)$ & $\mathrm{n}(\%)$ & $n(\%)$ & $\mathrm{n}(\%)$ & $n(\%)$ \\
\hline Overall & $301(50)$ & $300(50)$ & $368(61)$ & $233(39)$ & $1,035(100)$ \\
\hline Age, mean (SD) & $66.0 \mathrm{y}(10.0)$ & $66.1 y(10.6)$ & $64.7 \mathrm{y}(10.1)$ & $68.2 \mathrm{y}(10.3)$ & $64.2 y(12.2)$ \\
\hline \multicolumn{6}{|l|}{ Sex } \\
\hline $\begin{array}{l}\text { Male } \\
\text { Female }\end{array}$ & $\begin{array}{l}101(34) \\
200(66)\end{array}$ & $\begin{array}{r}88(29) \\
212(71) \\
\end{array}$ & $\begin{array}{l}113(31) \\
255(69)\end{array}$ & $\begin{array}{r}76(33) \\
157(67) \\
\end{array}$ & $\begin{array}{l}401(39) \\
634(61) \\
\end{array}$ \\
\hline Current smoker & $23(8)$ & $28(9)$ & $34(9)$ & $17(7)$ & $168(16)$ \\
\hline \multicolumn{6}{|l|}{ Race/cthnicity } \\
\hline Non-Hispanic & $292(97)$ & $295(98)$ & $359(98)$ & $228(98)$ & $1,004(97)$ \\
\hline White & $259(86)$ & $247(82)$ & $316(86)$ & $190(82)$ & $855(83)$ \\
\hline Black & $29(10)$ & $32(11)$ & $30(8)$ & $31(13)$ & $55(5)$ \\
\hline BMI. mcan (SD) & $29.1(5.7)$ & $29.2(6.7)$ & $29.4(6.5)$ & $28.8(5.7)$ & $30.4(6.1)$ \\
\hline \multicolumn{6}{|l|}{ Comorbiditics } \\
\hline Diabetes & $39(13)$ & $41(14)$ & $48(13)$ & $32(14)$ & $168(16)$ \\
\hline Hypertension & $141(47)$ & $134(45)$ & $164(45)$ & $111(48)$ & $651(63)$ \\
\hline
\end{tabular}

Demographics between the NSQIP and SPORT groups were similar (Table 1). NSQIP patients more often underwent decompression without fusion (13\% vs 5\% SPORT). If fused, NSQIP patients were less likely to be instrumented (65\% vs $73 \%$ SPORT). Of patients undergoing instrumented fusion, NSQIP patients were more likely to have interbody fusion ( $51 \%$ vs $17 \%$ SPORT).

Most perioperative factors and complications were similar: average operative time within $0.4 \mathrm{~min}$, wound complications within $1 \%$, postoperative transfusion within $3 \%$, and death within $1 \%$. However, average length of stay differed by 2.1 days (SPORT longer).

Discussion: When comparing SPORT degenerative spondylolisthesis surgical patients to the demographically similar NSQIP cohort, some important similarities and differences were identified. The greatest differences between the SPORT spondylolisthesis surgical population and the matched NSQIP population were that interbody fusions were three times as common in the NSQIP population, and length of stay was on average 2.1 days shorter.

Conclusion: Important differences in surgical procedure distribution between NSQIP and SPORT indicate that the degenerative spondylolisthesis arm of the SPORT trial is similar, but not completely generalizable, to national numbers.

\section{Poster 47. Sagittal Spinopelvic Alignment in Skeletally Mature Patients with Scheuermann's Disease}

Marcin Tyrakowski; Steven Mardjetko; Kris Siemionow

The Center of Postgraduate Medical Education, Department of Orthopaedics, Pediatric Orthopaedics and Traumatology

Disclosures: M. Tyrakowski: None. S. Mardjetko: 1; I; Spinecraft. 4; I; Depuy. 6; I; Spinecraft. K. Siemionow: 1; I; Amedica. 2; I; Globus; DePuy. 4; I; Amedica; LifeSpine. 8; I; Medtronic.

Introduction: Sagittal spinopelvic alignment has already been characterized in normal subjects and deviations in sagittal parameters have been reported for various spinal pathologies. No study has investigated spinopelvic parameters in Scheuermann's disease (SD).

Aim: The aim of the study was to analyze anatomical and positional parameters of spinopelvic sagittal alignment in mature patients with SD.

Methods: Standing postero-anterior and lateral radiographs of the skeletally mature patients with SD were analyzed retrospectively. Immature patients and these with other spinal pathologies were excluded from the study. Pelvic positional and anatomic parameters and spinal parameters were measured. Pelvic incidence (PI) was compared to the values reported for healthy individuals (t-test). Correlations between the measured parameters were analyzed and quantified by Pearson's linear correlation coefficient (r).
Results: Forty-five patients met the inclusion criteria. Sixteen females and 29 males in the mean age of 23 years were analyzed. There were 29 patients with the typical (thoracic) form of SD and 16 with the atypical (thoracolumbar) form. The mean PI in all of the patients was $41^{\circ}$ and was significantly lower than reported for healthy adults and adolescents $(\mathrm{p}<0.0001)$ and not significantly different from reported for healthy children $(\mathrm{p}=0.24)$. Patients with atypical SD had significantly lower PI than these with typical form $\left(43^{\circ}\right.$ vs. $\left.37^{\circ} ; \mathrm{p}=0.041\right)$. The difference in PI between females and males with SD was not significant $\left(40^{\circ}\right.$ vs. $41^{\circ}$; $\mathrm{p}=0.55)$. There was negligible correlation between PI and lumbar lordosis $(\mathrm{r}=0.16 ; \mathrm{p}=0.31)$, thoracic kyphosis $(\mathrm{r}=-0.14 ; \mathrm{p}=0.35)$ or thoracolumbar kyphosis ( $\mathrm{r}=-0.42 ; \mathrm{p}=0.041 *)$.

Conclusions: This study demonstrated that skeletally mature patients with SD have significantly lower PI than healthy adults. There was no correlation between PI and lumbar lordosis in individuals with SD. This challenges the role of PI in predicting the proper values of lumbar lordosis in this group of patients. Further studies are needed to investigate if impaired spinopelvic alignment is a cause or a result of SD.

Poster 48. The Effect of Psychological Distress on Functional Outcome Scores from Lumbar Spine Surgery

Prokopis Annis, MD; William R. Spiker, MD; Brandon D. Lawrence, MD; Michael D. Daubs, MD; Darrel S. Brodke, MD

\section{University of Utah, Orthopaedic Surgery}

Disclosures: P. Annis: None. W.R. Spiker: None. B.D. Lawrence: None. M.D. Daubs: 1; I; Synthes Spine. 4; I; Synthes Spine. D.S. Brodke: 1; I; Amedica, DePuy Synthes, Medtronic. 4; I; DePuy Synthes. 6; I; Amedica, Vertiflex.

Introduction: Psychological distress has been shown to affect the perception of pain and treatment outcomes in spine disorders. This study evaluates surgical outcomes of patients considered distressed, at-risk or normal on a validated psychological screening questionnaire (DRAM).

Methods: After IRB approval, 134 consecutive patients at one institution requiring surgical intervention for a degenerative lumbar spine condition underwent routine evaluation including a history and physical, the Oswetry Disability Index (ODI), Visual Analog Score (VAS) for back and leg and the Distress Risk Assessment Method (DRAM).

Results: Patients with psychological distress self reported much more disability (ODI) and increased back pain (VAS) than their non-distressed peers (ODI: 60 vs 34.82, p<0.001 VAS Back: 7.2 vs 5.6; $\mathrm{p}=0.004)$. All patients, regardless of the level of psychological stress (distressed, at-risk or non-distressed) experienced similar and significant improvement in disability (ODI) $(\mathrm{P}<0.001)$ (See table 1).

However, distressed patients showed decreased improvement in both back and leg pain as measured by VAS post-operatively (Distressed: VAS Back: -1.8, VAS Leg -1.9; Non-Distressed: VAS Back=-2.7, VAS Leg=-3.1, $\mathrm{p}<0.2$ for VAS Back and $\mathrm{p}=0.15$ for VAS Leg). Among at risk and distressed patients, surgery was shown to improve the level of distress in approximately $47 \%$ of patients with $9 \%$ of patients experiencing increased distress post operatively $(\mathrm{P}<0.001)$. 


\section{Abstracts of the 2014 Meeting of the Lumbar Spine Research Society}

\begin{tabular}{|c|c|c|c|c|c|c|c|c|c|c|c|c|}
\hline \multirow[t]{2}{*}{ LUMBAR } & \multicolumn{3}{|c|}{ ALL $=134$} & \multicolumn{3}{|c|}{ Normalyz 45} & \multicolumn{3}{|c|}{ At Risk 60} & \multicolumn{3}{|c|}{ Distressed=29 } \\
\hline & $\mathrm{N}$ & Mean & Std D & N & Mean & Std D & $\mathrm{N}$ & Mean & Std D & $\mathrm{N}$ & Mean & Std D \\
\hline PreOp OOI & 134 & 43.18 & 17.15 & 45 & 34.82 & 14.42 & 60 & 41.32 & 15.05 & 29 & 60 & 13.44 \\
\hline Post Op ODI & 133 & 28.42 & 19.72 & 45 & 2204 & 17.52 & 59 & 27.07 & 16.76 & 29 & 41.07 & 23.11 \\
\hline ODI Change & 133 & -14.77 & 18.27 & 45 & -12.78 & 18.36 & 59 & -14.24 & 16 & 29 & -18.93 & 22.1 \\
\hline $\begin{array}{l}\text { PreOp VAS } \\
\text { Back }\end{array}$ & 134 & 43.18 & 2237 & 45 & 5.633 & 2312 & 54 & 6.085 & 2.187 & 28 & 7.179 & 1.926 \\
\hline $\begin{array}{l}\text { Post Op VAS } \\
\text { Back }\end{array}$ & 133 & 28.42 & 2998 & 43 & 2942 & 2.542 & 58 & 3255 & 3.017 & 28 & 5.286 & 3.074 \\
\hline $\begin{array}{l}\text { VAS Back } \\
\text { Change }\end{array}$ & 133 & -14.77 & 3042 & 44 & -2.727 & 2.496 & 53 & 294 & 3.079 & 27 & -1.815 & 3.688 \\
\hline $\begin{array}{l}\text { PreOp Leg } \\
\text { VAS }\end{array}$ & 118 & 5.222 & 2667 & 42 & 5.196 & 2359 & 47 & 5.181 & 2765 & 29 & 5.328 & 3001 \\
\hline $\begin{array}{l}\text { Post Op Leg } \\
\text { VAS }\end{array}$ & 127 & 2.446 & 2.76 & 43 & 2044 & 2579 & 56 & 2286 & 2.628 & 28 & 3.382 & 3.15 \\
\hline $\begin{array}{l}\text { VAS Leg } \\
\text { Change }\end{array}$ & 113 & .2692 & 3.197 & 41 & 3.101 & 354 & 44 & -2845 & 2596 & 28 & -1.85 & 3.473 \\
\hline
\end{tabular}

cant morbidity and economic burden with an estimated prevalence of $8-27 \%$, resulting in over 37,000 Medicare surgeries per year. Often, a concomitant spinal fusion is performed with or without instrumentation. While instrumentation has been shown to generate higher fusion rates, its impact on improved patient reported outcomes is unclear. Furthermore, there is a significant cost associated with its use as well as an increased rate of complications.

Methods: A single center followup survey study was conducted at a tertiary-care teaching hospital. A single surgeon's database identified all LSS with spondylolisthesis patients who underwent surgical treatment between 2002 and 2010. Adult patients with

Discussion: Psychological distress has been shown to be common among patients with degenerative spinal conditions and can affect the perception of pain and disability. This study shows that surgical intervention in distressed individuals can improve self-rated disability to a similar degree seen in non-distressed individuals. However, improvements in back and leg pain are slightly decreased in distressed patients. Further, surgery in at-risk and distressed patients is approximately 5 times more likely to improve psychological distress than worsen distress.

Conclusion: Compared to their non-distressed counterparts, distressed patients experience similar improvements in their disability (ODI) but slightly decreased improvements in back and leg pain (VAS) post operatively. Patient education and expectation management is paramount in all patients, especially at-risk and distressed individuals.

Poster 49. Comparison Between Instrumented And Uninstrumented Posterolateral Fusion For Lumbar Spinal Stenosis And Spondylolisthesis

Alexander Richter; Alexander Richter, MD, MS; Stelios Koutsoumbelis, MD; David A. Essig, MD; Sara Merwin, MPH; Matthew J. Goldstein, MD; Jeff S. Silber, MD

\section{North Shore Long Island Jeweish, Orthopaedic Surgery}

Disclosures: A. Richter: None. A. Richter: None. S. Koutsoumbelis: None. D.A. Essig: None. S. Merwin: None. M.J. Goldstein: None. J.S. Silber: None.

Introduction: Lumbar spinal stenosis (LSS) is a source of signifi-

\begin{tabular}{|c|c|c|c|}
\hline & Instrumented & Uninstrumented & P-value \\
\hline Age & 58.1 & 67.9 & $<0.001$ \\
\hline Sex & & & \\
\hline Male & 17 & 21 & \\
\hline Female & 42 & 29 & \\
\hline $\begin{array}{c}\text { Length of } \\
\text { Follow-Up }\end{array}$ & 6.2 & 6.02 & 0.670 \\
\hline Pre-Op VAS & & & \\
\hline Back & 8.65 & 8.65 & 0.992 \\
\hline Leg & 7.43 & 7.14 & 0.641 \\
\hline Post-Op VAS & & & \\
\hline Back & 3.81 & 3.38 & 0.410 \\
\hline Leg & 3.07 & 3.22 & 0.777 \\
\hline ODI & 22.8 & 44.67 & 0.128 \\
\hline Repeat Sx & 2 & 3 & 0.530 \\
\hline
\end{tabular}
ICD 9 codes for lumbar spinal stenosis and spondylolisthesis were included in the analysis. Patients' under the age of 18, revision surgeries, and those with a less than two-year follow-up period were excluded. Questionnaires consisting of demographic information, Oswestry Disability Index (ODI), VAS for back and leg pain, and medication usage profiles were mailed to patients meeting inclusion criteria. A second mailing and telephone administration targeted nonrespondents.

Results: Of the 243 patients who met the inclusion criteria, 109 responded to the survey. 59 were Instrumented Fusions (IF) and 50 were Uninstrumented Fusions (UF). Length of follow-up was 6.2 and $6.0(\mathrm{p}=0.67)$ for the IF and UF groups. Age was significantly different in the IF and UF groups (58.1 and 67.9; $\mathrm{p}<0.001)$. There was no significant difference in the pre or postoperative Back or Leg pain VAS between the two groups. However, each group demonstrated significant improvements in both Back and Leg pain VAS. ODI at final follow-up trended to be better in the instrumented group but no significant difference was found. Medication usage was similar between the groups (42.4\% in the IF and $48 \%$ in the UF).

Conclusion/Discussion: In both the IF and UF groups, there was a significant reduction in both Back and Leg pain at final follow-up. Less than $5 \%$ of patients required further surgery. These results suggest similar outcomes can be achieved with regards to the reduction of back and leg pain with either instrumented or uninstrumented fuions for the treatment of lumbar stenosis with concomitant spondylolisthesis in carefully selected patients. Use of uninstrumented fusions may reduce overall medical costs and complication rates. Further studies are needed to determine which patients may best benefit from the use of instrumentation.

Poster 50. Evidence of an Inherited Predisposition for Spinal Cord Tumors

William R. Spiker, MD; Brandon D. Lawarence, MD; Prokopis Annis, MD; Darrel S. Brodke, MD; Lisa Cannon-Albright, PhD

\section{University of Utah, Orthopaedic Surgery}

Disclosures: W.R. Spiker: None. B.D. Lawarence: None. P. Annis: None. D.S. Brodke: 1; I; Amedica, DePuy Synthes, Medtronic. 4; I; DePuy Synthes. 6; I; Amedica, Vertiflex. L. Cannon-Albright: None.

Introduction: A genetic predisposition for the development of Spinal Cord Tumors has not yet been reported in the English speaking literature. The combination of a computerized Utah genealogy database with over fifteen years of clinical diagnosis data from the Utah Cancer Registry has allowed examination of the familial clustering of spinal cord tumors.

Methods: The Utah Population Database (UPDB) contains the 


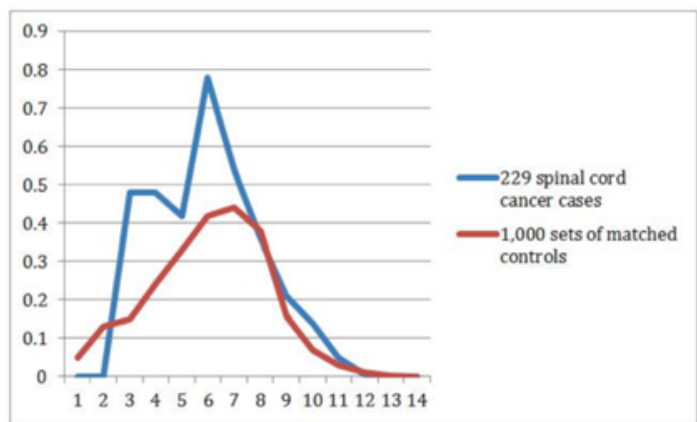

Figure 1: Contribution to the GIF statistic by genetic distance showing an increased relatedness of cases (X-Axis = Genetic distance of pairs; Y-Axis = Contribution to the GIF statistic)

combined health and genealogic data on over 2.3 million Utah residents. ICD-9 codes were used to identify 229 patients in Utah Cancer Registry with a diagnosis of a spinal cord tumor (ICD9 code 192.2 - Malignant Neoplasm of Spinal Cord). The hypothesis of excessive familial clustering was tested using the Genealogical Index of Familiality (GIF) and Relative risks (RR) in relatives by comparing rates of disease in relatives with rates estimated in the relatives of 5 matched controls for each case. This methodology has been previously reported for other disease conditions but not for spinal cord tumors.

Results: The GIF in patients with spinal cord tumors showed a trend of excess relatedness for disease $(\mathrm{p}=0.07)$ and a significantly significant excess relatedness for disease in distant relationships $(\mathrm{p}=0.02)$. The relative risks were elevated in $2 \mathrm{nd}$ and $3 \mathrm{rd}$ degree relatives (1.9 and 2.0) further supporting a familial predisposition to spinal cord tumors.

Conclusion: Excess relatedness of cases and elevated risks to distant relatives was observed. This data supports a heritable predisposition to spinal cord tumors.

\section{Poster 51. Comparison Between Laminectomy And Interspinous Devices For Treatment Of Lumbar Spinal Stenosis}

Alexander Richter, MD, MS; Stelios Koutsoumbelis, MD; David A. Essig, MD; Sara Merwin, MPH; Matthew J. Goldstein, MD; Jeff S. Silber, MD

\section{North Shore Long Island Jeweish, Orthopaedic Surgery}

Disclosures: A. Richter: None. S. Koutsoumbelis: None. D.A. Essig: None. S. Merwin: None. M.J. Goldstein: None. J.S. Silber: None.

Introduction: Lumbar spinal stenosis (LSS) is a source of significant morbidity and economic burden with an estimated prevalence of $8-27 \%$, resulting in over 37,000 Medicare surgeries per year. Laminectomy is often performed to treat LSS but interspinous device insertion is a less invasive option with potentially decreased operative times and patient risk but with the potential for device removal and laminectomy in the future. Studies comparing long-term outcomes have been scarce.

Methods: A single center follow-up survey study was conducted at a tertiary-care teaching hospital. A single surgeon's database identified all LSS patients who underwent surgical treatment between 2002 and 2010. Adult patients with ICD 9 codes for lumbar spinal stenosis were included in the analysis. Patients' under the age of 18 , revision surgeries, and those with a less than two-year follow-up period were excluded. Questionnaires consisting of demographic information, Oswestry Disability Index (ODI), VAS for back and leg pain, and medication usage profiles were mailed to patients meeting inclusion criteria. A second mailing and telephone administration targeted nonrespondents.

\begin{tabular}{|r|c|c|c|}
\hline & Laminectomy & Interspinous Device & P-value \\
\hline Age & 57 & 72.7 & $<0.001$ \\
\hline Sex & & & \\
\hline Male & 31 & 26 & \\
\hline Female & 32 & 24 & \\
\hline $\begin{array}{r}\text { Length of } \\
\text { Follow-Up }\end{array}$ & 5.54 & 4.83 & 0.019 \\
\hline Pre-Op VAS & & & \\
\hline Back & 8.63 & 7.73 & 0.129 \\
\hline Leg & 8.47 & 6.76 & 0.002 \\
\hline Post-Op VAS & & & \\
\hline Back & 3.46 & 3.76 & 0.316 \\
\hline Leg & 2.88 & 3.14 & 0.406 \\
\hline ODI & 26.59 & 33.84 & 0.981 \\
\hline Repeat Sx & 3 & 4 & 0.495 \\
\hline
\end{tabular}

Results: Of the 223 patients who met the inclusion criteria, 113 responded to the survey. 63 were Laminectomies (Lami) and 50 were intraspinous devices (ID). Length of follow-up was 5.5 and 4.8 $(\mathrm{p}=0.019)$ for the Lami and ID groups. Age was significantly different in the Lami and ID groups (57 and 72.7; $\mathrm{p}<0.001$ ). Preoperative Leg pain was significantly worse in the Lami group but there were no other significant differences in the pre or postoperative Back or Leg pain VAS between the two groups. Each group demonstrated significant improvements in both Back and Leg pain VAS. ODI at final follow-up was significantly better in the Lami group. Medication usage was similar between the groups (39.7\% in the Lami and 50\% in the ID).

Conclusion/Discussion: In both the Lami and ID groups, there was a significant reduction in both Back and Leg pain at final follow-up. However, Lami patients had a significantly better ODI. 4\% of Lami patients required further surgery compared to $8 \%$ of ID patients. These results suggest that both procedures lead to reduction of back and leg pain. However, Lami appears to result in improved ODI scores and decreased rate of reoperation. Further studies are needed to determine which patients may best benefit from laminectomy versus interspinous device insertion.

Poster 52. Revision Rate Following Thoracolumbar Fusion for Adult Deformity: Upper Thoracic versus Thoracolumbar UIV

Prokopis Annis, MD; Brandon D. Lawrence, MD; William R. Spiker, MD; Michael D. Daubs, MD; Darrel S. Brodke, MD

\section{University of Utah, Orthopaedics}

Disclosures: P. Annis: None. B.D. Lawrence: None. W.R. Spiker: None. M.D. Daubs: 1; I; Synthes Spine. 4; I; Synthes Spine. D.S. Brodke: 1; I; Amedica, DePuy Synthes, Medtronic. 4; I; DePuy Synthes. 6; I; Amedica, Vertiflex.

Introduction: Complication rates are relatively high in adult deformity surgery. While some authors report that nonunion is the most common complication leading to revision surgery, other think proximal junctional failure (PJF) more often leads to revision. The rate of PJF may vary based on the level of the upper-instrumented vertebrae (UIV), and non-union may vary based on number of levels fused. The purpose of this study was to review and compare revision rates of fusions with UIV in the upper thoracic (UT) or thoracolumbar (TL) spine.

Methods: We reviewed 216 consecutive patients with minimum 2-year follow up, treated operatively for deformity at a single institution. Patients were divided into 2 cohorts, those with fusion to the UT 


\section{Abstracts of the 2014 Meeting of the Lumbar Spine Research Society}

\begin{tabular}{|l|c|c|c|c|}
\hline Pts & ALL=216 & UT=72 & TL=144 & p value $^{*}$ \\
\hline All Revisions & $74 / 216(34.3)$ & $26 / 72(36.1)$ & $48 / 144(33.3)$ & 0.76 \\
\hline 2 Years & $56 / 216((25.9)$ & $20 / 72(27.8)$ & $37 / 144(25.7)$ & 0.7458 \\
\hline Final F-Up & $18 / 216(8.3)$ & $6 / 72(8.3)$ & $11 / 144((7.6)$ & 1 \\
\hline PJFs Revisions & & & & \\
\hline All & $28 / 216(13)$ & $2 / 72(2.8)$ & $26 / 144(18)$ & 0.001 \\
\hline 2 Years & $22 / 216(10.2)$ & $2 / 72(2.8)$ & $21 / 144(14.6)$ & 0.0087 \\
\hline Final F-Up & $6 / 216(2.8)$ & $0 / 72(0)$ & $5 / 144(3.5)$ & 0.172 \\
\hline Nonunion Revisions & & & & \\
\hline All & $30 / 216(14)$ & $21 / 72(29.2)$ & $9 / 144(6.3)$ & $<0.0001$ \\
\hline 2 Years & $22 / 216(10.2)$ & $15 / 72(20.8)$ & $7 / 144(4.9)$ & 0.0005 \\
\hline Final F-Up & $8 / 216(3.7)$ & $6 / 72(8.3)$ & $2 / 144(1.4)$ & 0.0178 \\
\hline
\end{tabular}

spine (T1-T5) and TL spine (T7-L2). Revision rates were calculated at 24 months and at the final follow-up.

Results: 216 patients with mean age 61 years (18-86) and mean follow-up 43 months (24-126) were reviewed. 72 patients had the UIV in the UT spine (33.3\%), and 144 patients had the UIV in the TL spine $(66.7 \%)$. Revision was most commonly required for nonunion in the UT group $(20.8 \%)(\mathrm{p}<0.001)$, and for PJF in the TL spine $(14.6 \%)$ $(p<0.009)$. There were no differences in absolute revision rate for the UT and TL patients at 2-year and final follow up, though early revision (within the first year) was more often required for PJF in the TL spine. Late revision rates for nonunion were significantly higher in the UT group $(\mathrm{p}=0.018)$. Other causes of revision were symptomatic adjacent segment disease (8.3\%) and coronal imbalance (2.8\%). Age over 55 was a significant risk factor for PJF and nonunion revision.

Conclusion: Adult deformity surgery has a relatively high complication rate with revisions commonly required due to PJF or nonunion. The complication profile varies based on the location of the UIV. While revision for PJF was significantly lower in those fused to the upper thoracic spine (2.8\%), the nonunion rate required revision was significantly higher $(29.2 \%)$ negating any perceived benefit of the longer fusion. Older age was also a significant predictor for revision surgery.

\section{Poster 53. Reoperation and Immediate Readmission Rates after Circumferential Minimally Invasive Surgical (cMIS) Correction of Adult Spinal Deformities (ASD)}

Neel Anand, MD, Mch Orth; Babak Khandehroo, MD; Eli Baron, MD; Sheila Kahwaty, PA-C

\section{Cedars-Sinai Medical Center, Spine Center}

Disclosures: N. Anand: 1; I; Medtronics, Globus Medical, Nuvasive. 2; I; TranS1, Medtronics, Globus Medical. 4; I; TranS1, Medtronics, Globus Medical. 6; I; TranS1, Globus Medical. B. Khandehroo: None. E. Baron: None. S. Kahwaty: None.

Introduction: Hospital readmission rate has been an important measure of quality and cost-effectiveness of care. Several studies have shown that postoperative complications and readmission represent a starting point for quality improvement efforts in any surgery. However, there is little information about the readmission following MIS correction of ASD. We assessed reoperation and admission rate after cMIS correction of ASD.

Methods: A retrospective review of patients who underwent cMIS correction of thoracolumbar deformity between January 2007 and May 2013 included 216pts who were grouped by fusion length: group 1:short fusion (2-3 levels, $n=102$ ), group 2: medium fusion(4-8 levels, $n=96$ ) and group 3: long fusion(9 or more, $n=18$ ). Deformities were included: Adult Scoliosis(147), Degenerative Disc Disorder(51), Kyphosis(8) and Spondylolisthesis(10). Our primary interest was

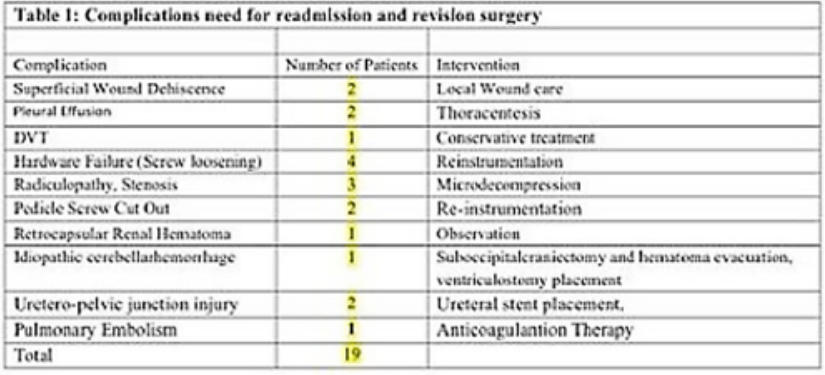

30-day postoperative readmission, defined as any admission within 30 days of the index operation.

Results: mean age 61yrs(21-85). An average of 5 levels (2-16) was fused. Mean hospital stay was 7days(1-27). The 30-day postoperative complication rate in our study was $8.8 \%(\mathrm{n}=19)$ including: 3 complication during surgery, 6 during post-op hospital stay, 2 in the first week, 2 in the 2 nd week, 2 in the 3 rd week and 4 in the 4 thweek postoperatively. Out of these 19 post-op complications, 9 patients needed corrective or revised surgery during initial hospitalization: 1 with Idiopathic cerebellar hemorrhage, 1 with Retrocapsular Renal Hematoma, 2 with Uretero-pelvic junction injury, 2 with Wound dehiscence, 1 pleural effusion, 1 with Pulmonry embolism and 1 with hardware failure. Within 30 days after index surgery, 10 patients needed readmission: 3 pts with radiculopathy and stenosis, 2 pts with pedicle screw loosening and cut out, 3pts with hardware failure, 1 with pleural effusion and one with Deep Vein Thrombosis. Overall 30-day readmission rate was $4.6 \%(\mathrm{n}=10)$, which in group 1 was $4.9 \%(\mathrm{n}=5)$, in group 2 was $4.1 \%(\mathrm{n}=4)$ and in group 3 was $5.5 \%(\mathrm{n}=1)$.

Conclusions: Our study delivers significant evidence that efforts to reduce hospital readmissions for ASD patients should begin by concentrating on postoperative complications. cMIS approaches will not eliminate all postoperative readmissions but will likely have a major effect on dropping complication rates which could result in a substantial reduction in readmissions. 
Abstracts of the 2014 Meeting of the Lumbar Spine Research Society 